\title{
A Neophyl Palladacycle as an Air- and Thermally Stable Precursor to Oxidative Addition Complexes
}

\author{
Ryan P. King, ${ }^{1}$ Shane W. Krska, ${ }^{2}$ and Stephen L. Buchwald ${ }^{1 *}$ \\ ${ }^{1}$ Department of Chemistry, Massachusetts Institute of Technology, Cambridge, Massachusetts \\ 02139, United States \\ ${ }^{2}$ Merck \& Co., Inc., Kenilworth, New Jersey 07033, United States \\ *Correspondence to: sbuchwal@mit.edu
}

Supporting Information 


\section{Table of Contents}

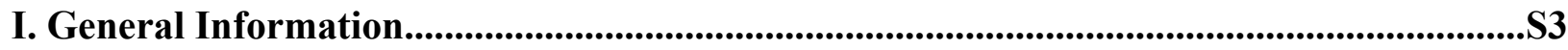

II. Synthesis of Pd OAC Precursors.............................................................................................S8

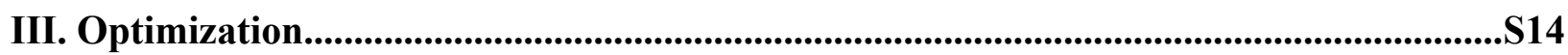

IV. Synthesis of Oxidative Addition Complexes and other Pd Complexes from P3..........S15

V. P3 as a Precatalyst for Pd-Catalyzed Cross-Coupling Reactions...................................S35

VI. Stability Studies.....................................................................................................................S37

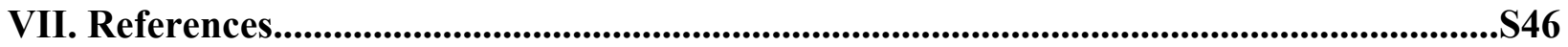

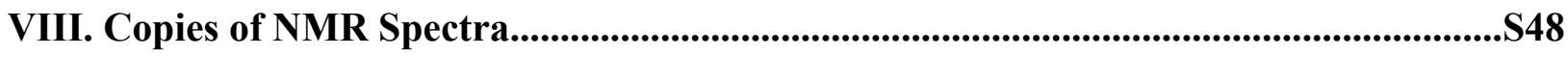




\section{General Information}

\section{General Analytical Information}

All compounds were characterized by ${ }^{1} \mathrm{H}$ NMR, ${ }^{13} \mathrm{C}$ NMR, ${ }^{19} \mathrm{~F}$ NMR (when applicable), ${ }^{31} \mathrm{P}$ NMR (when applicable), Infrared (IR) spectroscopy, and melting point analysis. All new compounds were also characterized by either elemental analysis (EA) or high-resolution mass spectrometry (HRMS). Nuclear Magnetic Resonance spectra were recorded on a Bruker 400, 500, or $600 \mathrm{MHz}$ instrument. All NMR spectra are recorded in $\delta$ units and parts per million (ppm). The multiplicities are abbreviated with s (singlet), br s (broad singlet), d (doublet), t (triplet), q (quartet), and hept (heptet). All ${ }^{1} \mathrm{H}$ and ${ }^{13} \mathrm{C}$ spectra were calibrated using residual solvent as an internal reference $\left(\mathrm{CDCl}_{3}: \delta 7.26 \mathrm{ppm}\right.$ and $\delta 77.16 \mathrm{ppm}$ respectively; $\mathrm{C}_{6} \mathrm{D}_{6}: \delta 7.16 \mathrm{ppm}$ and $\delta 128.06 \mathrm{ppm}$ respectively; $\mathrm{CD}_{3} \mathrm{CN}: \delta 1.94 \mathrm{ppm}$ and $\delta 118.26 \mathrm{ppm}$ respectively; $\mathrm{CD}_{2} \mathrm{Cl}_{2}: \delta 5.32 \mathrm{ppm}$ and $\delta 53.84$ ppm respectively). ${ }^{1}$ All ${ }^{19} \mathrm{~F}$ NMR spectra were calibrated to an external standard of trifluorotoluene $\left(\mathrm{PhCF}_{3}\right)$ in $\mathrm{CDCl}_{3}(\delta-63.72 \mathrm{ppm})$. All ${ }^{31} \mathrm{P} \mathrm{NMR}$ spectra were calibrated to an external standard of triphenylphosphine in $\mathrm{CDCl}_{3}(\delta-4.90 \mathrm{ppm})$. All ${ }^{13} \mathrm{C}$ NMR and ${ }^{31} \mathrm{P}$ NMR spectra were obtained with ${ }^{1} \mathrm{H}$ NMR decoupling. Elemental analyses were performed by Atlantic Microlabs Inc., Norcross, GA, USA. All HRMS were recorded on an Agilent Technologies 6545 Q-TOF LC/MS system. All IR spectra were obtained on a Thermo Scientific Nicolet iS5 spectrometer (iD5 ATR, diamond) and were reported in wavenumbers $\left(\mathrm{cm}^{-1}\right)$. Melting points were obtained using a Stanford Research Systems EZ-melt melting point apparatus. All melting points herein described as decomposed (dec.) were compounds that turned black and melted over the reported range. LC/MS analysis was performed with a Thermo Scientific Accucore C18 column $(30 \times 2.1 \mathrm{~mm}$, $2.6 \mu \mathrm{m}$ particle size) maintained at $45{ }^{\circ} \mathrm{C}$ within an instrument consisting of Agilent 1260 series binary pump and an Agilent 6120 quadrupole MS operating in positive MM-ES+APCI ionization mode. Flash column chromatography was performed using SiliCycle SiliaFlash ${ }^{\circledR}$ F60 silica gel

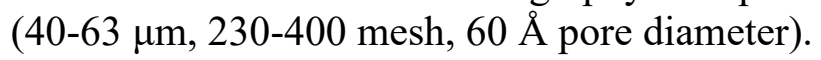

\section{General Reagent Information}

Reagent information: All reagents were purchased from commercial sources and used as received unless otherwise noted. Palladium(II) chloride $\left(\mathrm{PdCl}_{2}\right)$ was purchased from Johnson Matthey. 1,5cyclooctadiene (cod), 2-methyl-2-phenylpropylmagnesium chloride (0.5 M in diethyl ether, SureSeal ${ }^{\mathrm{TM}}$ ), (trimethylsilyl)methylmagnesium chloride $\left(1.0 \mathrm{M}\right.$ in diethyl ether, Sure-Seal $\left.{ }^{\mathrm{TM}}\right)$, zinc chloride $\left(\mathrm{ZnCl}_{2}, 1.9 \mathrm{M}\right.$ in 2-MeTHF, Sure-Seal $\left.{ }^{\mathrm{TM}}\right)$, 3-bromoquinoline, morpholine, and $i$ propylmagnesium chloride (2.0 M in THF, Sure-Seal $\left.{ }^{\mathrm{TM}}\right)$ were purchased from Millipore-Sigma. Tert-ButylXPhos ( $t$-BuXPhos), XPhos, SPhos, RuPhos, tert-ButylBrettPhos ( $t$-BuBrettPhos), and BrettPhos were gifts from Millipore-Sigma, for which we are grateful. Silica gel (SiliaFlash ${ }^{\circledR}$ F60 silica gel, 40-63 $\mu \mathrm{m}, 230-400$ mesh, $60 \AA$ pore diameter) was purchased from SiliCycle. X4 was obtained from Merck \& Co., Inc. Deuterated solvents were purchased from Cambridge Isotope Laboratories and dried over activated molecular sieves (4 $\AA$ ) overnight prior to use. 4Chloroanisole was purchased from Tokyo Chemical Industry Co. 4-Bromobenzotrifluoride was purchased from Matrix Scientific. Rivaroxaban, gefitinib, and 1,3,5-trimethoxybenzene were purchased from Combi-Blocks. Sodium tert-butoxide $(\mathrm{NaOt}-\mathrm{Bu})$ was purchased from Strem and stored in a $\mathrm{N}_{2}$-filled glovebox. Portions were removed from the glovebox in an oven-dried $20 \mathrm{~mL}$ scintillation vial, stored in a desiccator box, and used within 3 days. 
Solvent information: ethanol (EtOH, 200 proof, ACS reagent grade) and tetrahydrofuran (THF, stabilized, ACS Reagent Grade, SKU: 360589) for reactions were purchased from MilliporeSigma and used as received. Isopropanol ( $i-\mathrm{PrOH}, \mathrm{HPLC}$ grade), pentane (reagent grade, SKU: 158941) and diethyl ether (stabilized, SKU: 673811) for purifications were purchased from Millipore-Sigma and used as received. $n$-hexane (anhydrous, Sure-SealTM, SKU: 296090) for reactions was purchased from Millipore-Sigma and degassed prior to use by placing under vacuum for $10 \mathrm{~s}$, sonicating, and replenishing with an atmosphere of nitrogen gas. This cycle was repeated 3 times. Cyclohexane (anhydrous, Sure-Seal ${ }^{\mathrm{TM}}$, SKU: 227048) and 2-methyltetrahydrofuran (2MeTHF, anhydrous, Sure-Seal ${ }^{\mathrm{TM}}$, SKU: 673277) for reactions were purchased from MilliporeSigma and degassed by sparging with argon for $30 \mathrm{~min}$ prior to use. Tetrahydrofuran (THF, anhydrous), diethyl ether (Et $2 \mathrm{O}$, anhydrous), and dichloromethane $\left(\mathrm{CH}_{2} \mathrm{Cl}_{2}\right)$ for reactions were purchased from J.T. Baker in CYCLE-TAINER ${ }^{\circledR}$ delivery kegs and purified by passing through two packed columns of neutral alumina and copper(II) oxide successively under an argon atmosphere prior to use.

4-(trifluoromethyl)phenyl trifluoromethanesulfonate, ${ }^{2}$ 2-ethyl-6-methylpyridin-3-yl trifluoromethanesulfonate, ${ }^{3}$ 2-(trimethylsilyl)ethyl 4-bromobenzoate, ${ }^{4}$ 2,5-dioxopyrrolidin-1-yl 3(4-bromophenyl)propanoate, ${ }^{5}$ perfluorophenyl 4 -bromobenzoate, ${ }^{6}$ AlPhos, ${ }^{7}$ sSPhos,${ }^{8}$ CPhos, ${ }^{9}$ GPhos, ${ }^{10}$ and AdCyBrettPhos ${ }^{11}$ were prepared according to literature procedures.

\section{General Reaction Information}

-Reactions performed under argon involved addition of solids to air-sensitive reaction mixtures. In lieu of argon, nitrogen can be used along with appropriate Schlenk glassware.

-For THF and $\mathrm{Et}_{2} \mathrm{O}$, both solvents from a solvent system under argon and from $4 \mathrm{~L}$ bottles stored in air were used. In specific procedures, THF and $\mathrm{Et}_{2} \mathrm{O}$ from the solvent system are denoted as (anhydrous) and THF and $\mathrm{Et}_{2} \mathrm{O}$ from $4 \mathrm{~L}$ bottles in air are denoted as (stabilized).

-Organomagnesium and organozinc reagents were titrated with 1:1 $\mathrm{I}_{2}$ : $\mathrm{LiCl}$ in THF (anhydrous, $0.25 \mathrm{M}$ ) within one week of using.

-Glassware denoted as oven-dried was placed in an oven at $140{ }^{\circ} \mathrm{C}$ for at least $16 \mathrm{~h}$ and then allowed to cool to room temperature prior to use.

-Unless otherwise noted, all products were dried in vacuo on a Schlenk line at room temperature overnight $(\sim 16 \mathrm{~h})$.

-The components for reactions involving $8 \mathrm{~mL}, 16 \mathrm{~mL}$, and $24 \mathrm{~mL}$ reaction tubes are described below. 


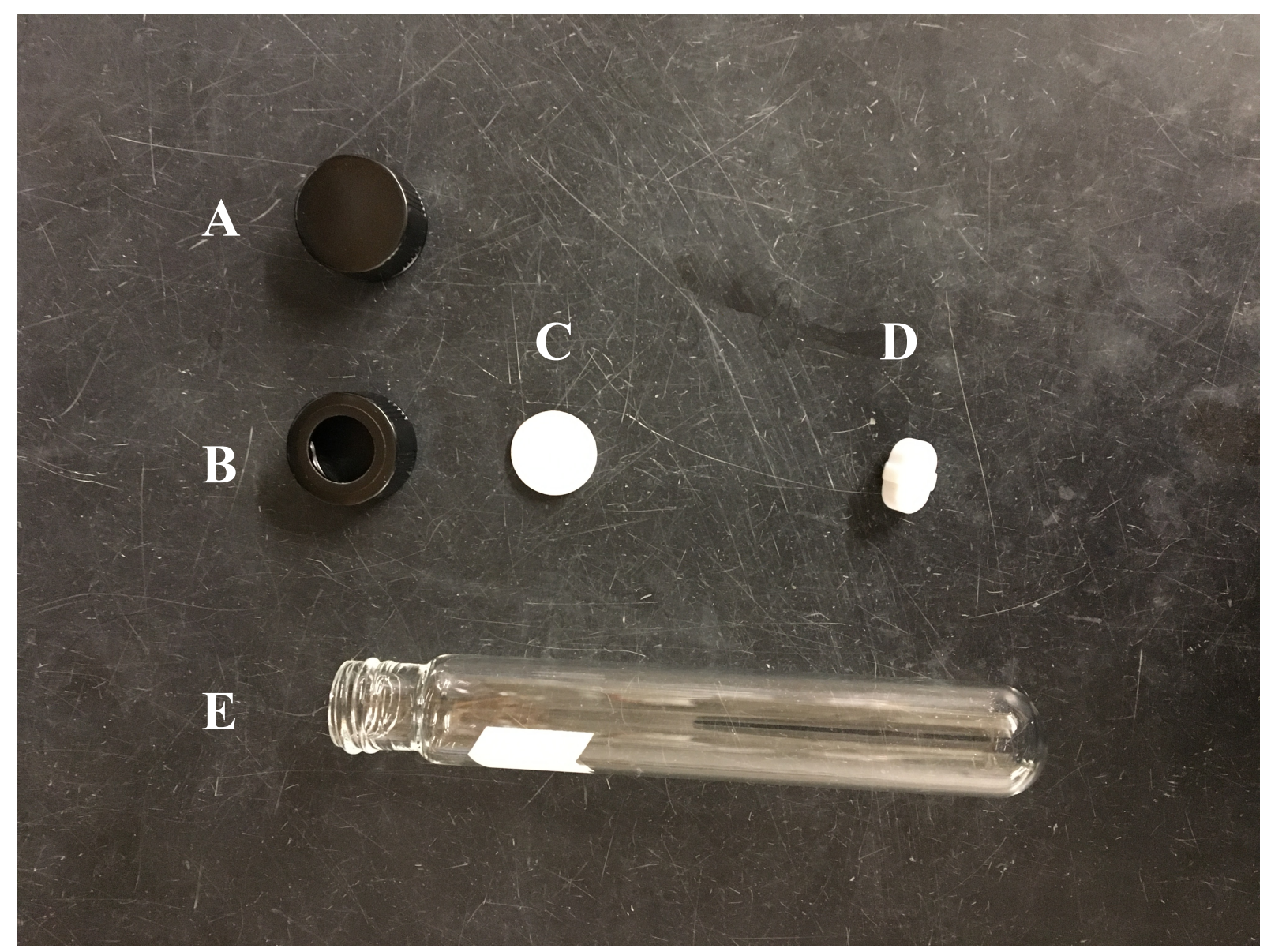

Figure S1: $24 \mathrm{~mL}$ reaction tube components

A. Kimble Chase 18-400 phenolic S/T cap, PTFE-faced rubber (cat. \# 73802-18400). Henceforth referred to as a solid cap (when applicable)

B. Kimble Chase 18-400 phenolic open top S/T cap (cat. \# 73804-18400). Henceforth referred to as a septum cap (when applicable)

C. Thermo Scientific PTFE-lined silicone septum (part \# C45015-60)

D. A small PTFE-coated magnetic stir bar

E. Fisher Scientific 20 x $125 \mathrm{~mm}$ (24 mL) disposable culture tube S/C (part \# 1495937A) 


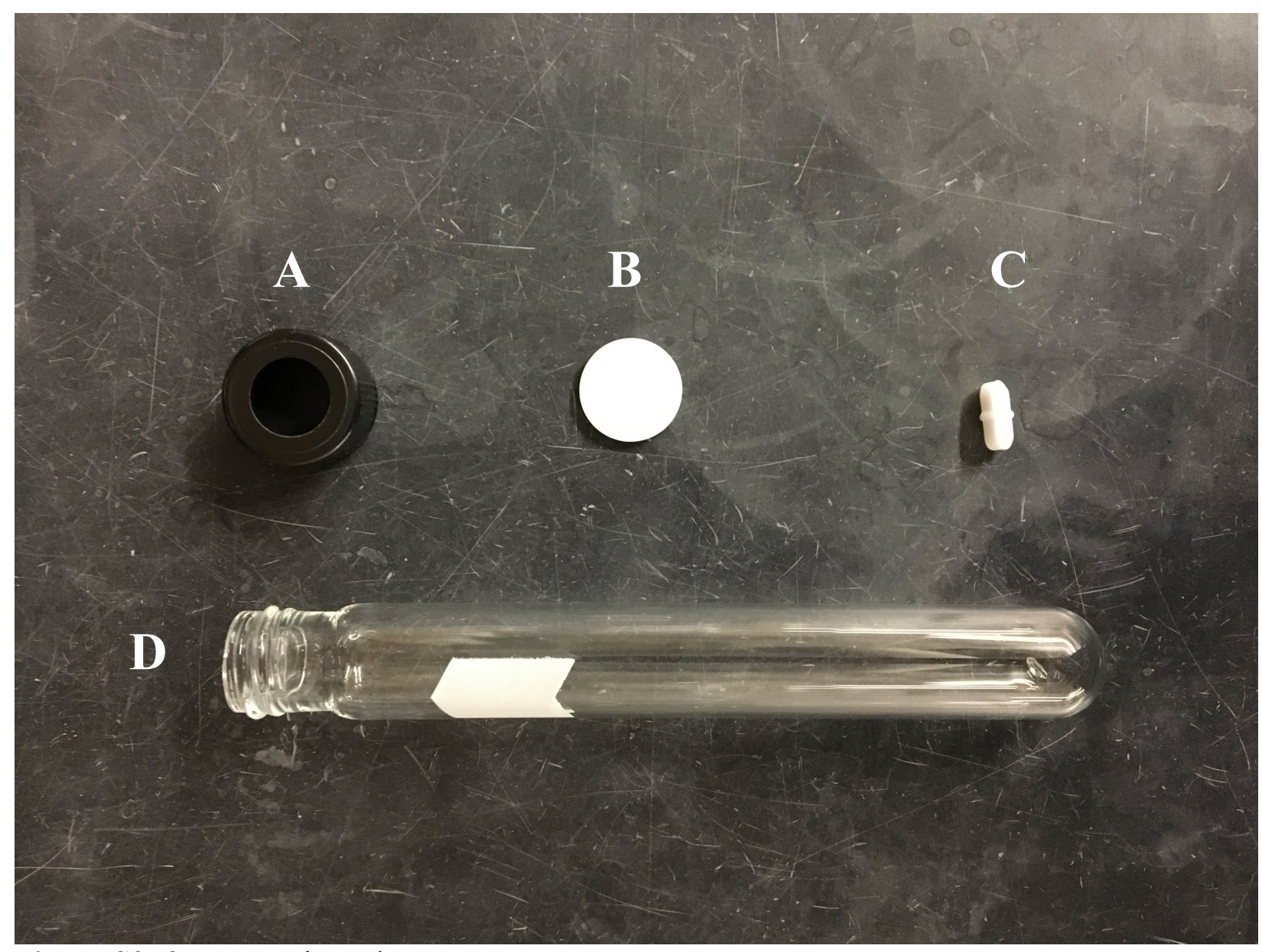

Figure S2: $8 \mathrm{~mL}$ reaction tube components

A. Thermo Scientific 13-425 phenolic cap (part \# C4014-66)

B. Thermo Scientific PTFE-lined silicone septum (part \# C45015-60)

C. A small PTFE-coated magnetic stir bar

D. Fisher Scientific 13 x $100 \mathrm{~mm}(8 \mathrm{~mL})$ disposable culture tube S/C (part \# 1495935C) 


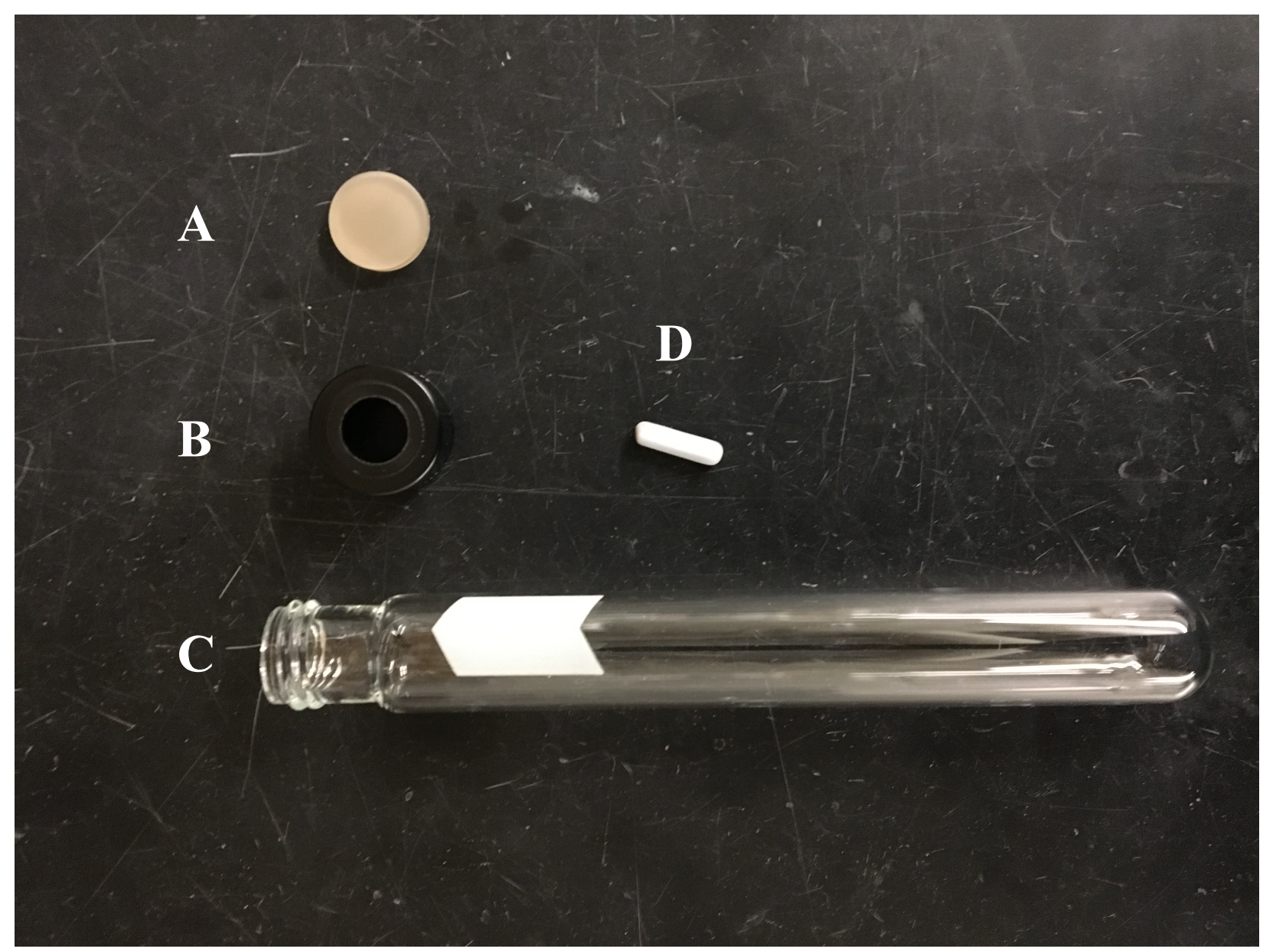

Figure S3: $16 \mathrm{~mL}$ reaction tube components

A. Thermo Scientific PTFE-lined silicone septum (part \# B7995-15)

B. Kimble Chase 15-425 phenolic open top S/T cap (cat. \# 73804-15425)

C. Fisher Scientific 16 x $125 \mathrm{~mm}$ (16 mL) disposable culture tube S/C (part \# 1496226G)

D. A small PTFE-coated magnetic stir bar 


\section{Synthesis of Palladium OAC Precursors}

\section{$(\operatorname{cod}) \mathrm{PdCl}_{2}(\mathrm{~S} 1)$}<smiles>ClP(Cl)(Cl)(C1CC2CCC1C2)C1CC2CCC1C2</smiles>

S1

S1 was made according to a modified literature procedure. ${ }^{12}$ A $100 \mathrm{~mL}$ roundbottom flask equipped with a magnetic stir bar was charged with $\mathrm{PdCl}_{2}(10.00 \mathrm{~g}$, $56.4 \mathrm{mmol}, 1.0$ equiv). In air the flask was charged with concentrated $\mathrm{HCl}(10.0$ $\mathrm{mL})$. The red-colored suspension was allowed to stir for $10 \mathrm{~min}$ at room temperature. At this time the suspension was charged with EtOH $(50 \mathrm{~mL})$ and was allowed to stir for $10 \mathrm{~min}$. After $10 \mathrm{~min}$, the mixture was filtered through a $2 \mathrm{~cm}$ plug of Celite ${ }^{\circledR}$ into a $500 \mathrm{~mL}$ round-bottom flask. The Celite ${ }^{\circledR}$ plug was further washed with EtOH $(3 \times 60 \mathrm{~mL})$. A magnetic stir bar was added to the flask containing the filtrate, followed by cod (28 mL, $230 \mathrm{mmol}, 4.0$ equiv). Upon addition of cod, a yellow precipitate rapidly formed. The flask was capped with a plastic stopper and the reaction mixture was allowed to stir for 30 min. The precipitate was then collected on a fritted funnel and washed with $\mathrm{Et}_{2} \mathrm{O}(3 \times 60 \mathrm{~mL})$ to yield S1 (15.77 g, 98\%) as a bright yellow powder. The ${ }^{1} \mathrm{H}$ NMR and ${ }^{13} \mathrm{C}$ NMR spectroscopic data matched that reported in the literature. ${ }^{12}$

${ }^{1}$ H NMR $\left(500 \mathrm{MHz}, \mathrm{CDCl}_{3}\right) \delta 6.36-6.27(\mathrm{~m}, 4 \mathrm{H}), 2.99-2.85(\mathrm{~m}, 4 \mathrm{H}), 2.63-2.51(\mathrm{~m}, 4 \mathrm{H})$. ${ }^{13} \mathbf{C ~ N M R}\left(126 \mathrm{MHz}, \mathrm{CDCl}_{3}\right) \delta 116.8,31.1$.

IR (neat): 3010, 2987, 2942, 2880, 1523, 1511, 1481, 1445, 1417, 1341, 1310, 1246, 1232, 1177 , $1085,1012,995,905,863,844,822,792,765,678 \mathrm{~cm}^{-1}$

M.P.: $245-250{ }^{\circ} \mathrm{C}($ dec.)

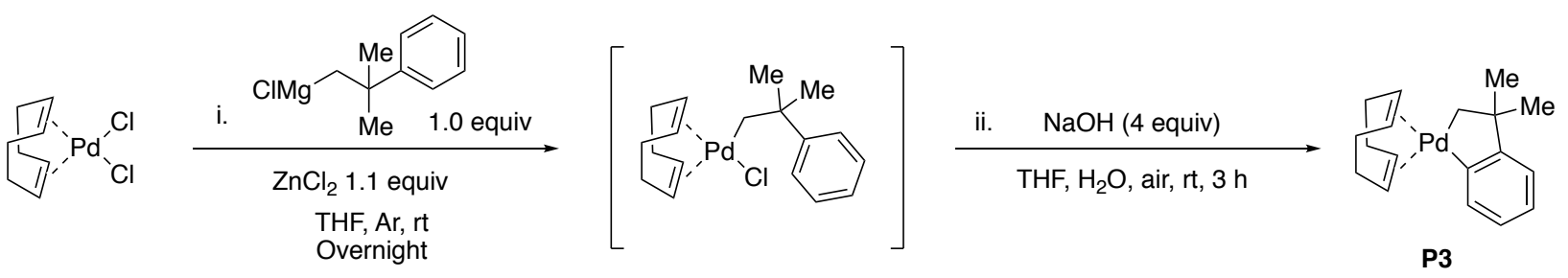

$(\operatorname{cod}) \mathrm{Pd}\left(\mathrm{CH}_{2} \mathrm{CMe}_{2} \mathrm{C}_{6} \mathrm{H}_{4}\right)(\mathrm{P3})$

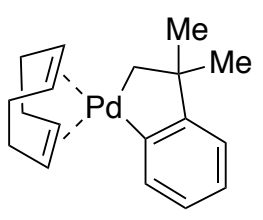

P3

25 mmol scale from $\mathrm{Zn}$ : A $500 \mathrm{~mL}$ round-bottom flask equipped with a magnetic stir bar was sealed with a rubber septum. The septum was pierced with a needle connected to a dual manifold Schlenk line with a rubber hose and the flask was placed under vacuum. The flask was flame-dried and allowed to cool to room temperature under vacuum before backfilling with argon. The flask was charged with $\mathrm{ZnCl}_{2}$ (1.9 M in 2-MeTHF, $14.4 \mathrm{~mL}, 27.5$ mmol, 1.1 equiv) followed by THF (anhydrous, $300 \mathrm{~mL}$ ) via syringe. The solution was subsequently charged with 2-methyl-2-phenylpropylmagnesium chloride $(0.85 \mathrm{M}$ in $\mathrm{Et}_{2} \mathrm{O}, 29.4 \mathrm{~mL}, 25.0 \mathrm{mmol}, 1.0$ equiv) as a slow, steady stream over 2 min via syringe, during which time a white precipitate formed (Figure S4A). The solution was allowed to stir for $30 \mathrm{~min}$. At this time, the septum was quickly removed and (cod) $\mathrm{PdCl}_{2}(7.14 \mathrm{~g}, 25.00 \mathrm{mmol}, 1.0$ equiv) was added in one portion under a counterflow of argon. The flask was quickly resealed with the rubber septum and the light brown-colored slurry (Figure S4B) was allowed to stir at room temperature overnight $(\sim 16 \mathrm{~h})$, during which time the reaction mixture turned into a grey-brown-colored slurry (Figure S4C). At this time, the flask was opened to air and the heterogeneous mixture was filtered 
through a $2 \mathrm{~cm}$ plug of Celite ${ }^{\circledR}$ (packed in a $150 \mathrm{~mL}$ fritted funnel) into a $1 \mathrm{~L}$ round-bottom flask. The Celite ${ }^{\circledR}$ plug was further washed with THF (stabilized, $450 \mathrm{~mL}$ ) (Figure S4D). A magnetic stir bar was added to the flask containing the yellow-orange-colored filtrate, followed by water (7.5 $\mathrm{mL}$ ) and $\mathrm{NaOH}$ (freshly ground, $4.00 \mathrm{~g}$ ) (Figure S4E). The flask was capped with a plastic stopper and the reaction mixture was stirred for $3 \mathrm{~h}$ at room temperature, during which time the yelloworange-colored solution turned cloudy dull yellow-brown (Figure S4F). At this time, the solution was concentrated to dryness in vacuo with the assistance of a rotary evaporator (Figure S4G). The resulting residue was suspended in $\mathrm{Et}_{2} \mathrm{O}$ (stabilized, $500 \mathrm{~mL}$ ) and filtered through a $1.5 \mathrm{~cm}$ plug of Celite ${ }^{\circledR}$ (packed in a $150 \mathrm{~mL}$ fritted funnel). The Celite ${ }^{\circledR}$ plug was further washed with $\mathrm{Et}_{2} \mathrm{O}$ (stabilized, $250 \mathrm{~mL}$ ) (Figure $\mathrm{S} 4 \mathbf{H}$ ). The yellow-orange-colored filtrate was concentrated to dryness in vacuo with the assistance of a rotary evaporator. The resulting orange powder was suspended in cold pentane $\left(\sim 0{ }^{\circ} \mathrm{C}, 60 \mathrm{~mL}\right)$, and the suspension was triturated with the assistance of sonication $(\sim 1 \mathrm{~min})$ to yield an off-white powder. The powder was collected via vacuum filtration on a fritted funnel and washed with cold pentane $\left(\sim 0{ }^{\circ} \mathrm{C}, 120 \mathrm{~mL}\right)$ (Figure S4I) to yield P3 (5.29-5.60 g, 61-65\%) as an off-white powder (Figure S4J).

A second crop of $\mathbf{P 3}$ was obtained by concentrating the filtrate in vacuo with the assistance of a rotary evaporator. The resulting red oil was charged with cold pentane $\left(\sim 0^{\circ} \mathrm{C}, 10 \mathrm{~mL}\right)$ and the flask was capped with a plastic stopper and placed in a freezer at $-25^{\circ} \mathrm{C}$. Over the course of $1 \mathrm{~h}$, a precipitate formed. The precipitate was collected on a fritted funnel and washed with cold pentane $\left(\sim-25{ }^{\circ} \mathrm{C}, 10 \mathrm{~mL}\right)$ to yield $\mathbf{P 3}(338-371 \mathrm{mg}, 4 \%)$ as a tan powder. Combined total: 5.66 $-5.94 \mathrm{~g}, 65-69 \%$. The ${ }^{1} \mathrm{H}$ NMR and ${ }^{13} \mathrm{C}$ NMR spectroscopic data matched that reported in the literature. $^{13}$

${ }^{1}$ H NMR $\left(500 \mathrm{MHz}, \mathrm{C}_{6} \mathrm{D}_{6}\right) \delta 7.27(\mathrm{dd}, J=7.2,1.5 \mathrm{~Hz}, 1 \mathrm{H}), 7.22(\mathrm{td}, J=7.3,1.4 \mathrm{~Hz}, 1 \mathrm{H}), 7.17-$ $7.15(\mathrm{~m}, 1 \mathrm{H}), 7.13(\mathrm{~d}, J=1.6 \mathrm{~Hz}, 1 \mathrm{H}), 5.71-5.65(\mathrm{~m}, 2 \mathrm{H}), 5.17(\mathrm{~m}, 2 \mathrm{H}), 2.53(\mathrm{~s}, 2 \mathrm{H}), 2.00-$ $1.79(\mathrm{~m}, 8 \mathrm{H}), 1.57(\mathrm{~s}, 6 \mathrm{H})$.

${ }^{13}$ C NMR (126 MHz, C $\left.6 \mathrm{D}_{6}\right) \delta 167.9,162.9,135.0,125.2,124.9,123.6,113.6,110.2,53.8,50.1$, 34.4, 29.3, 28.6.

M.P. $156-157^{\circ} \mathrm{C}$ (dec.)

IR (neat): 3043, 3003, 2965, 2942, 2918, 2889, 2866, 2833, 2781, 1568, 1473, 1457, 1445, 1433, 1421, 1367, 1346, 1312, 1286, 1249, 1224, 1199, 1182, 1154, 1121, 1083, 1033, 1019, 993, 976, $960,855,815,761,747,732,730,723,664,649,609 \mathrm{~cm}^{-1}$ 
A.

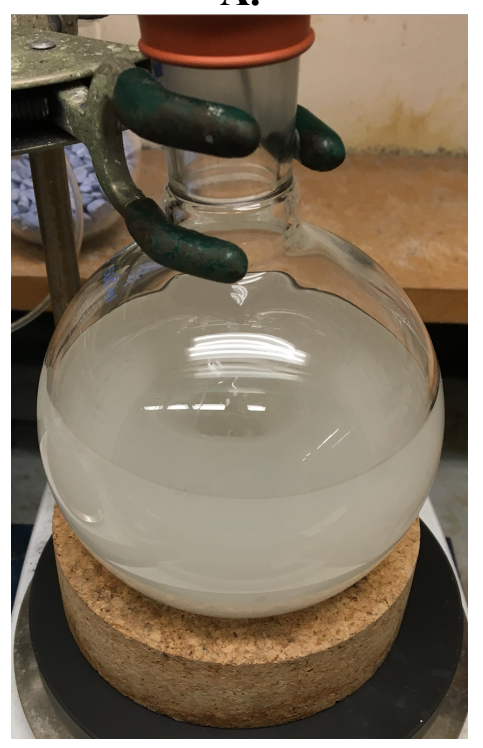

D.

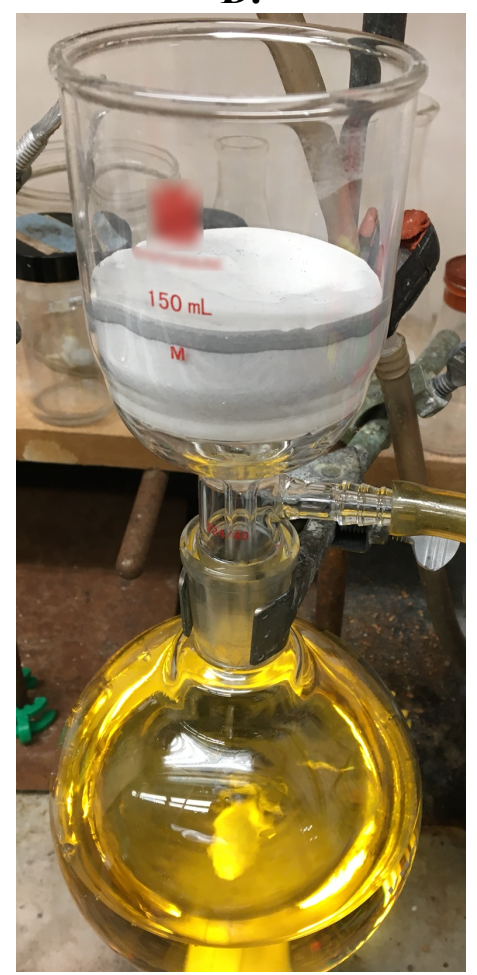

B.

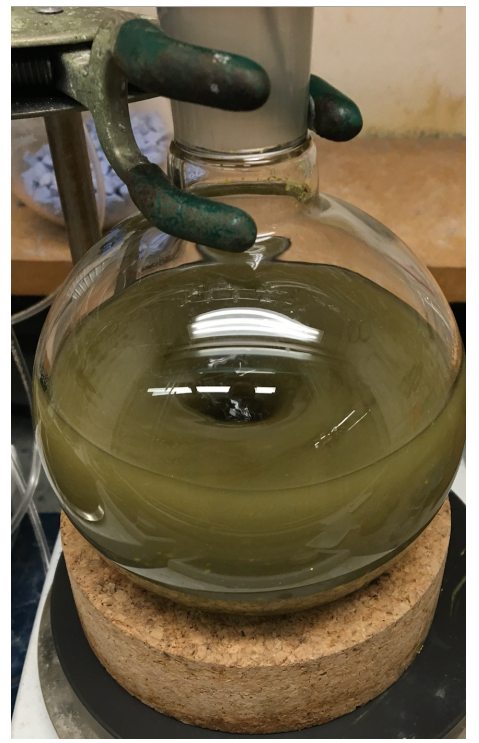

E.

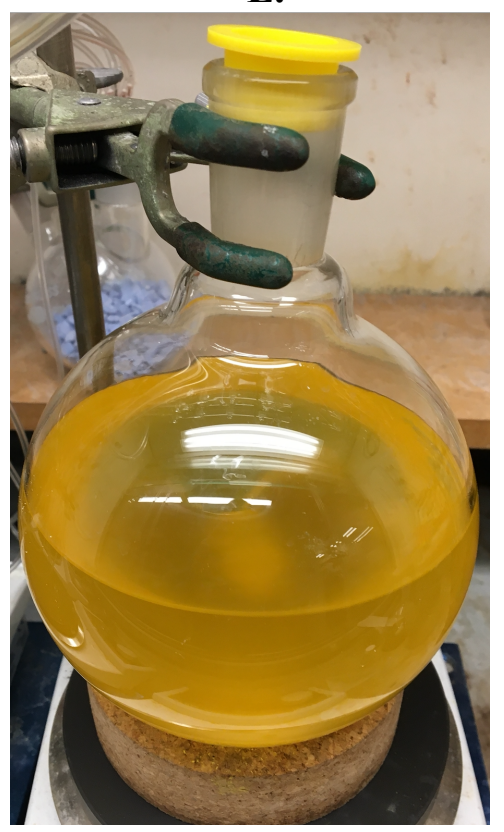

C.

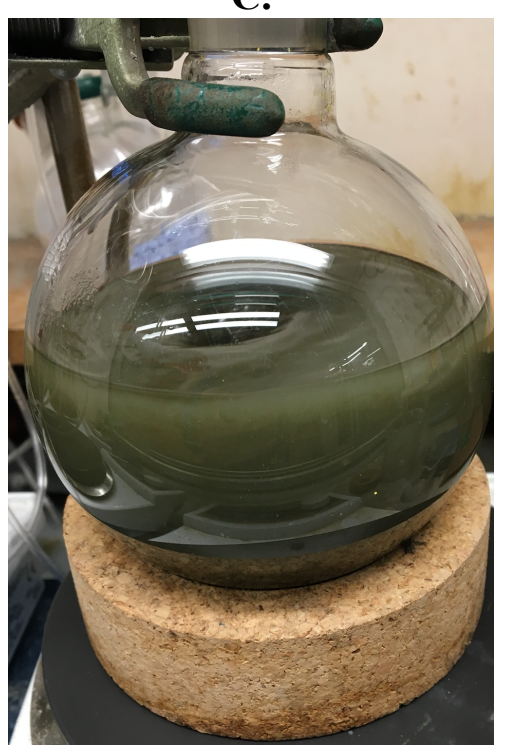

F.

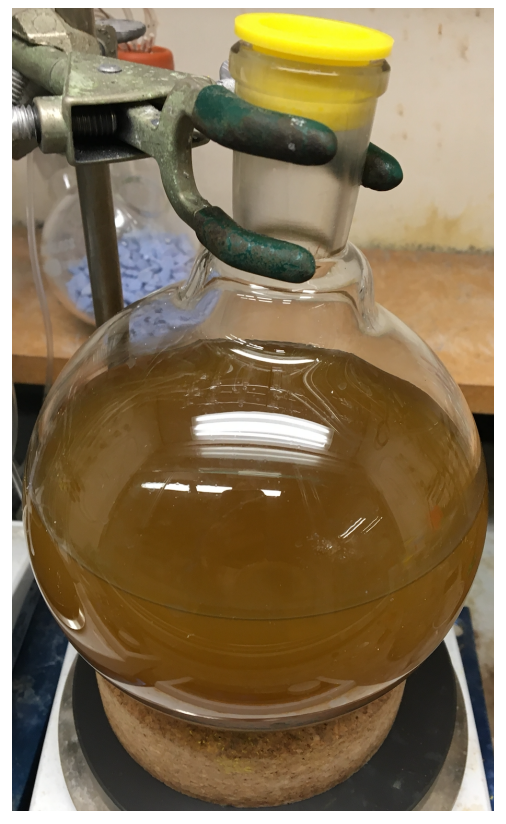


G.

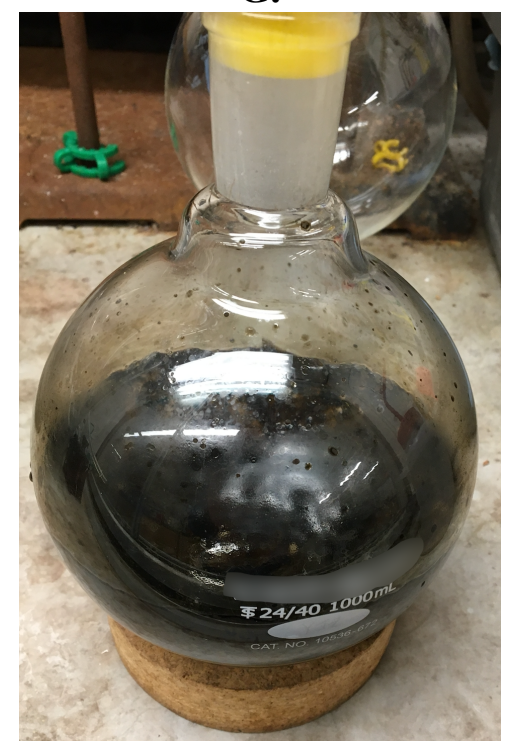

H.

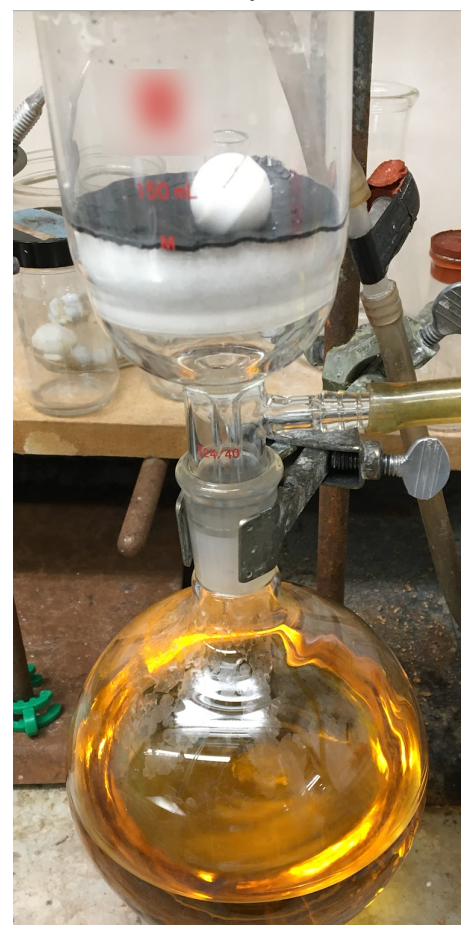

J.

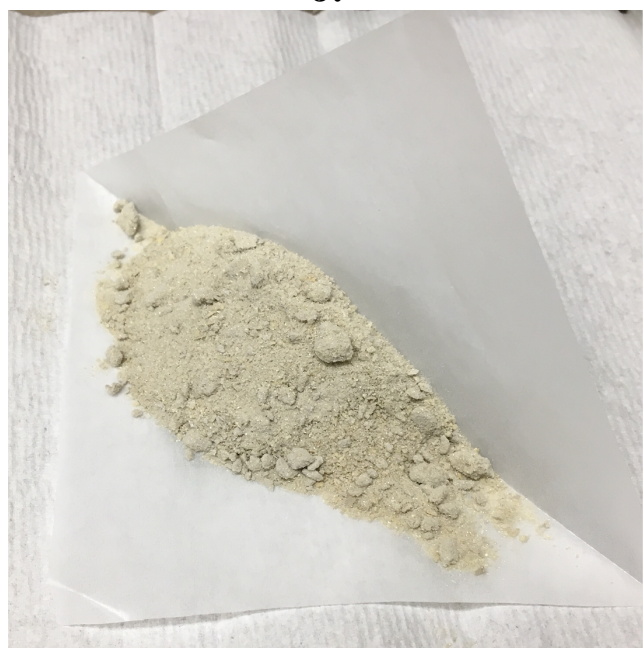

I.

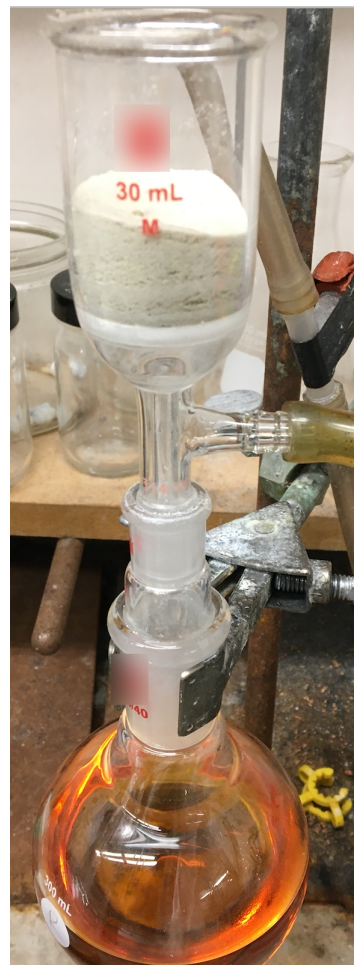

Figure S4. Preparation of $\mathbf{P 3}$ on $25 \mathrm{mmol}$ scale. A. Addition of Grignard to $\mathrm{ZnCl}_{2}$ solution. B. Solution immediately after the addition of $(\mathrm{cod}) \mathrm{PdCl}_{2}$. C. Solution after stirring overnight. D. Celite ${ }^{\circledR}$ filtration to remove insoluble $\mathrm{Mg}$ and $\mathrm{Zn}$ salts. E. Solution immediately after the addition of water and $\mathrm{NaOH}$. F. Solution after stirring for $3 \mathrm{~h}$ in the presence of $\mathrm{NaOH}$. G. Solution after concentration in vacuo. H. Celite ${ }^{\circledR}$ filtration to remove material insoluble in $\mathrm{Et}_{2} \mathrm{O}$. I. Pentane filtration to remove impurities from P3. The solid in the fritted funnel is pure $\mathbf{P 3}$. $\mathbf{J} . \mathbf{P 3}$ on $10 \mathrm{~cm}$ x $10 \mathrm{~cm}$ weigh paper. 
10 mmol scale from Mg: P3 was made according to a modified literature procedure. ${ }^{13} \mathrm{~A}$ flamedried $300 \mathrm{~mL}$ round-bottom flask equipped with a magnetic stir bar and rubber septum was allowed to cool to room temperature under vacuum with the aid of a dual manifold Schlenk line before backfilling with nitrogen. The rubber septum was removed and the flask was charged with (cod) $\mathrm{PdCl}_{2}$ (2.855 g, $10.0 \mathrm{mmol}, 1.0$ equiv). The flask was resealed with a rubber septum. The septum was pierced with a needle connected to a dual manifold Schlenk line with a rubber hose and the flask was evacuated before backfilling with nitrogen (the evacuation/backfill process was repeated a total of three times). $\mathrm{Et}_{2} \mathrm{O}$ (anhydrous, $120 \mathrm{~mL}$ ) was added via syringe and the yellowcolored suspension was cooled to $-78^{\circ} \mathrm{C}$ by submerging the flask in a dry ice/acetone cooling bath. After stirring at $-78{ }^{\circ} \mathrm{C}$ for $30 \mathrm{~min}$, 2-methyl-2-phenylpropylmagnesium chloride $(0.85 \mathrm{M}$ in $\mathrm{Et}_{2} \mathrm{O}, 11.76 \mathrm{~mL}, 10.0 \mathrm{mmol}, 1.0$ equiv) was added via syringe over $2 \mathrm{~min}$. After the addition was complete, the cooling bath was removed and the reaction mixture was allowed to stir overnight $(\sim$ $16 \mathrm{~h}$ ) at room temperature under nitrogen, during which time the yellow-colored reaction mixture became a grey-black-colored slurry. At this time, the flask was opened to air and the slurry was concentrated in vacuo with the assistance of a rotary evaporator. The resulting residue was suspended in THF (stabilized, $100 \mathrm{~mL})$ and filtered through a plug of silica $\left(\sim 30 \mathrm{~mL} \mathrm{SiO}_{2}\right.$ in a 60 $\mathrm{mL}$ fritted funnel) into a $500 \mathrm{~mL}$ round-bottom flask. The silica plug was further washed with THF (stabilized, $\sim 200 \mathrm{~mL}$ ). A magnetic stir bar was added to the flask containing the resulting brown-black-colored filtrate, followed by water $(3.0 \mathrm{~mL})$ and $\mathrm{NaOH}$ (freshly ground, $1.60 \mathrm{~g})$. The flask was capped with a plastic stopper and the reaction mixture was stirred for $3 \mathrm{~h}$ at room temperature, during which time the brown-black-colored solution turned cloudy and became yellow-brown in color. After $3 \mathrm{~h}$, the solution was concentrated to dryness in vacuo with the aid of a rotary evaporator. The resulting residue was suspended in $\mathrm{Et}_{2} \mathrm{O}$ (stabilized, $250 \mathrm{~mL}$ ) and filtered through a $1.5 \mathrm{~cm}$ plug of Celite ${ }^{\circledR}$ (packed in a $150 \mathrm{~mL}$ fritted funnel). The Celite ${ }^{\circledR}$ plug was further washed with $\mathrm{Et}_{2} \mathrm{O}$ (stabilized, $250 \mathrm{~mL}$ ). The yellow-orange-colored filtrate was concentrated to dryness in vacuo with the assistance of a rotary evaporator. The resulting orange powder was suspended in cold pentane $\left(\sim 0{ }^{\circ} \mathrm{C}, 25 \mathrm{~mL}\right)$, and the suspension was triturated with the assistance of sonication $(\sim 1 \mathrm{~min})$ to yield an off-white powder. The powder was collected via vacuum filtration on a fritted funnel and washed with cold pentane $\left(\sim 0{ }^{\circ} \mathrm{C}, 25 \mathrm{~mL}\right)$ to yield $\mathbf{P 3}$ $(2.46 \mathrm{~g}, 71 \%)$ as an off-white powder.

A second crop of $\mathbf{P 3}$ was obtained by concentrating the filtrate in vacuo with the assistance of a rotary evaporator. The resulting red oil was dissolved in cold pentane $\left(\sim 0{ }^{\circ} \mathrm{C}, 5 \mathrm{~mL}\right)$ and the flask was capped with a plastic stopper and placed in a freezer at $-25^{\circ} \mathrm{C}$. Over the course of $1 \mathrm{~h}$, a precipitate formed. The precipitate was collected on a fritted funnel and washed with cold pentane $\left(\sim-25{ }^{\circ} \mathrm{C}, 10 \mathrm{~mL}\right)$ to yield $\mathbf{P 3}(156 \mathrm{mg}, 4 \%)$ as a tan powder. Combined total yield: $\left.2.60 \mathrm{~g}, 75 \%\right)$.

\section{$(\operatorname{cod}) \mathrm{Pd}\left(\mathrm{CH}_{2} \mathrm{TMS}\right)_{2}(\mathrm{P} 1)$}

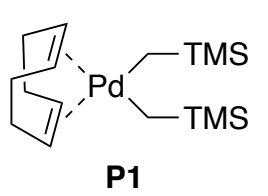

NOTE: Due to the thermal instability of the product, all manipulations were performed at $0^{\circ} \mathrm{C}$.

A flame-dried $300 \mathrm{~mL}$ round-bottom flask equipped with a magnetic stir bar was allowed to cool to room temperature under vacuum before being charged with (cod) $\mathrm{PdCl}_{2}(6.00 \mathrm{~g}, 21.0 \mathrm{mmol}, 1$ equiv). The flask was sealed with a rubber septum and connected to a dual manifold by piercing the rubber septum with a needle attached to a rubber hose. The flask was evacuated and backfilled with nitrogen (this process was repeated a total of three times), before being charged with $\mathrm{Et}_{2} \mathrm{O}$ (anhydrous, $30 \mathrm{~mL}$ ) via syringe. The yellow suspension was cooled to $0^{\circ} \mathrm{C}$ by submerging the 
flask in an ice/water bath. After stirring at $0{ }^{\circ} \mathrm{C}$ for $15 \mathrm{~min}$, (trimethylsilyl)methylmagnesium chloride (1.0 M, $42.0 \mathrm{~mL}, 42.0 \mathrm{mmol}, 2.0$ equiv) was added over $3 \mathrm{~min}$ via syringe. The solution was allowed to stir for $30 \mathrm{~min}$ at $0{ }^{\circ} \mathrm{C}$, during which time the slurry changed in color from yellow to brown. At this time, the flask was opened to air and the slurry was quenched by slow addition of acetone $(1 \mathrm{~mL})$ over $1 \mathrm{~min}$. The reaction mixture was concentrated to dryness in vacuo via rotary evaporation at $0{ }^{\circ} \mathrm{C}$. The residue with suspended in cold pentane $\left(0{ }^{\circ} \mathrm{C}\right)$ and filtered through Celite ${ }^{\circledR}$ into a receiving flask cooled to $0{ }^{\circ} \mathrm{C}$ with the aid of an ice/water bath. The Celite ${ }^{\circledR}$ pad was washed with cold pentane $\left(0^{\circ} \mathrm{C}, 3 \times 50 \mathrm{~mL}\right)$. The resulting colorless filtrate was concentrated to dryness in vacuo via rotary evaporation at $0{ }^{\circ} \mathrm{C}$. The resulting solid was transferred to a $20 \mathrm{~mL}$ scintillation vial, placed in an ice/water bath and dried in vacuo on the Schlenk line for $30 \mathrm{~min}$. P1 was obtained as a white to light grey crystalline solid $(6.50-7.49 \mathrm{~g}, 80-92 \%)$. The ${ }^{1} \mathrm{H}$ NMR and ${ }^{13} \mathrm{C}$ NMR spectroscopic data matched that reported in the literature. ${ }^{14}$

Note: This material must be stored at $-20{ }^{\circ} \mathrm{C}$ or below to avoid significant decomposition.

${ }^{1}$ H NMR $\left(600 \mathrm{MHz}, \mathrm{C}_{6} \mathrm{D}_{6}\right) \delta 5.15(\mathrm{~d}, J=3.6 \mathrm{~Hz}, 4 \mathrm{H}), 2.01-1.86(\mathrm{~m}, 8 \mathrm{H}), 0.73(\mathrm{~s}, 4 \mathrm{H}), 0.30$ (s, $18 \mathrm{H})$.

${ }^{13}$ C NMR $\left(151 \mathrm{MHz}, \mathrm{C}_{6} \mathrm{D}_{6}\right) \delta 112.2,29.3,11.7,4.0$.

\section{$(\operatorname{cod}) \mathrm{Pd}\left(2,4,6-\mathrm{F}_{3} \mathrm{Ph}\right)_{2}(\mathrm{P} 2)$}

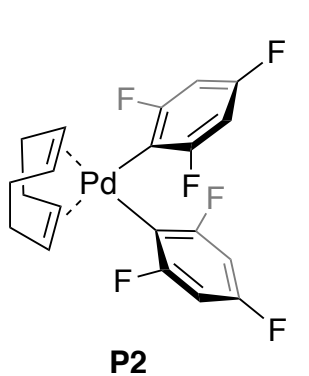

P2 was synthesized according to a modified literature procedure. ${ }^{15}$ An oven-dried $24 \mathrm{~mL}$ reaction tube was equipped, while still hot, with a magnetic stir bar and septum cap. The cap was pierced with a needle connected to a dual manifold via a rubber hose. The tube was allowed to cool to room temperature under vacuum before backfilling with argon. The tube was charged with 1-bromo-2,4,6-trifluorobenzene $(295 \mu \mathrm{L}, 2.50$ mmol, 2.50 equiv), followed by $\mathrm{Et}_{2} \mathrm{O}$ (anhydrous, $5 \mathrm{~mL}$ ) via syringe. The colorless solution was cooled to $0{ }^{\circ} \mathrm{C}$ by submerging the reaction tube in an ice/water bath. After stirring at $0{ }^{\circ} \mathrm{C}$ for $15 \mathrm{~min}$, $i$-propylmagnesium chloride (2.0 M in THF, $1.20 \mathrm{~mL}, 2.40 \mathrm{mmol}, 2.40$ equiv) was added via syringe over the course of $1 \mathrm{~min}$. Upon complete addition, the cooling bath was removed and the solution was allowed to stir for $2 \mathrm{~h}$ at room temperature. At this time, the cap was quickly removed and (cod) $\mathrm{PdCl}_{2}(286$ $\mathrm{mg}, 1.00 \mathrm{mmol}, 1.00$ equiv) was added as a solid. The tube was quickly recapped and the resulting slurry was stirred at room temperature overnight $(\sim 18 \mathrm{~h})$, during which time the yellow-colored slurry became a white-colored slurry. At this time, the cap was removed and the slurry was diluted with $\mathrm{Et}_{2} \mathrm{O}$ (stabilized, $5 \mathrm{~mL}$ ) and quenched with $\mathrm{H}_{2} \mathrm{O}(200 \mu \mathrm{L})$. The slurry was concentrated to dryness in vacuo with the assistance of a rotary evaporator. The resulting dark brown-colored solid was suspended in $\mathrm{CH}_{2} \mathrm{Cl}_{2}(10 \mathrm{~mL})$ and filtered through a $1 \mathrm{~cm}$ plug of Celite ${ }^{\circledR}$ and washed with $\mathrm{CH}_{2} \mathrm{Cl}_{2}(25 \mathrm{~mL})$ to afford a bright yellow-colored solution. The solution was concentrated to dryness in vacuo with the assistance of a rotary evaporator. The crude residue was suspended in isopropanol $(25 \mathrm{~mL})$, and the suspension was triturated with the assistance of sonication to yield a white powder. The powder was collected on a fritted funnel and washed with isopropanol $(\sim 25$ $\mathrm{mL})$ to yield $\mathbf{P 2}(300 \mathrm{mg}, 63 \%)$ as a white powder. The ${ }^{1} \mathrm{H} \mathrm{NMR},{ }^{13} \mathrm{C} \mathrm{NMR}$, and ${ }^{19} \mathrm{~F}$ NMR spectroscopic data matched that reported in the literature. ${ }^{15}$

${ }^{1} \mathbf{H}$ NMR $\left(600 \mathrm{MHz}, \mathrm{CDCl}_{3}\right) \delta 6.40-6.35(\mathrm{~m}, 1 \mathrm{H}), 5.78(\mathrm{~s}, 1 \mathrm{H}), 2.70(\mathrm{~s}, 1 \mathrm{H})$.

${ }^{13} \mathrm{C}$ NMR $\left(151 \mathrm{MHz}, \mathrm{CDCl}_{3}\right) \delta 165.1-164.6(\mathrm{~m}), 163.5-163.1(\mathrm{~m}), 162.5-162.2(\mathrm{~m}), 160.9-$ $160.6(\mathrm{~m}), 116.3,112.3-111.3(\mathrm{~m}), 99.3-98.6(\mathrm{~m}), 29.5$. 
${ }^{19}$ F NMR $\left(377 \mathrm{MHz}, \mathrm{CDCl}_{3}\right) \delta-86.83,-116.98$.

M.P. $167-168^{\circ} \mathrm{C}$ (dec.); discoloration seen at $123^{\circ} \mathrm{C}$. Lit.: $122^{\circ} \mathrm{C}$

IR (neat): 3098, 3015, 2946, 2927, 2887, 2842, 1679, 1642, 1616, 1587, 1500, 1478, 1452, 1433, $1405,1348,1312,1296,1284,1234,1154,1099,986,832,763,744,704,694,673,602,593,576$ $\mathrm{cm}^{-1}$

\section{Table 1}

\section{Optimization}

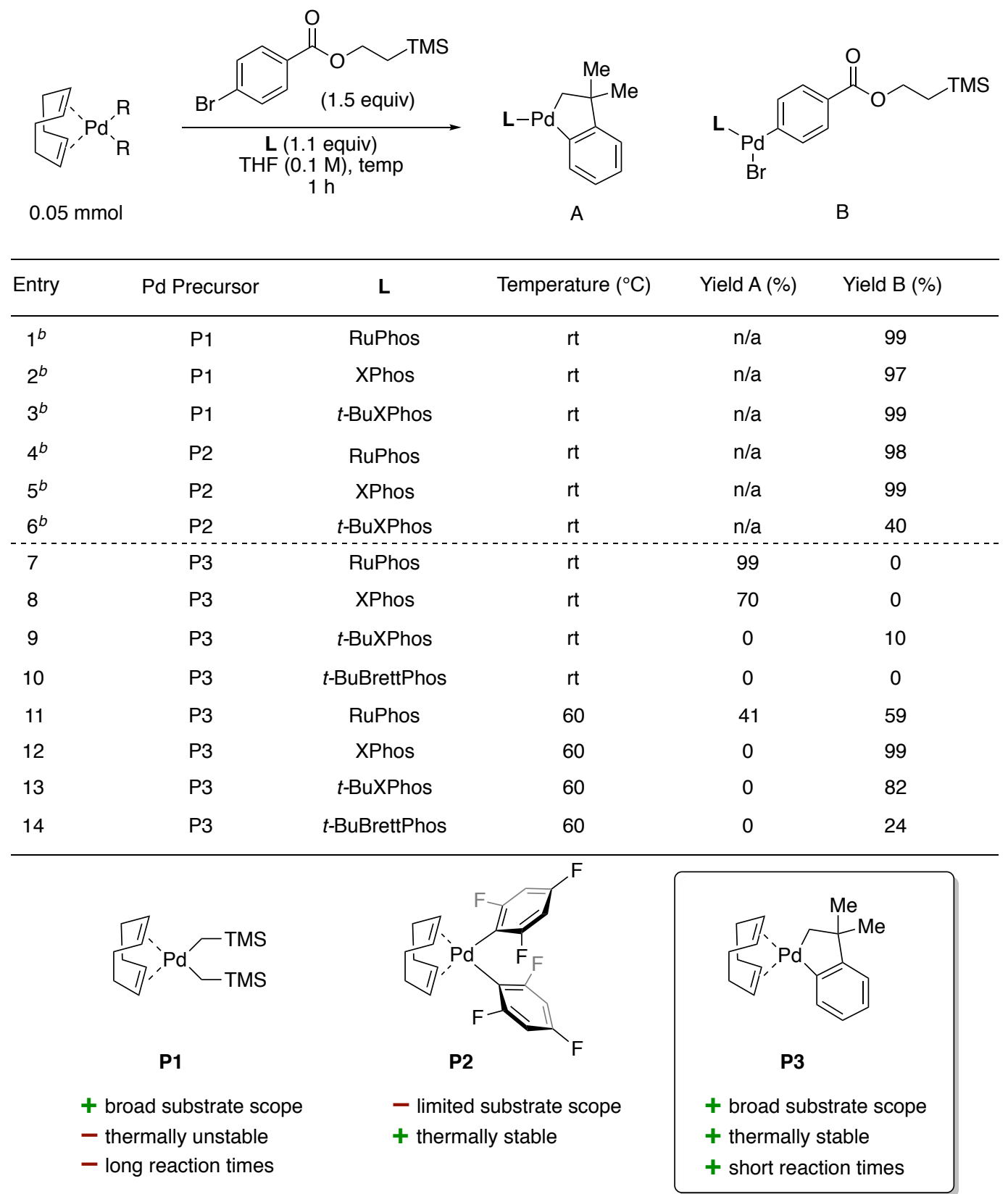

An oven-dried $8 \mathrm{~mL}$ reaction tube was equipped, while still hot, with a magnetic stir bar and septum cap. The flask was allowed to cool to room temperature under vacuum by piercing the septum with a needle connected to a dual manifold by a rubber hose. At this point, the cap was 
removed and the tube was charged with the corresponding Pd complex ( $0.050 \mathrm{mmol}, 1.00$ equiv), ligand ( $0.055 \mathrm{mmol}, 1.10$ equiv), 2-(trimethylsilyl)ethyl 4-bromobenzoate $(23 \mathrm{mg}, 0.075 \mathrm{mmol}$, 1.5 equiv), and 1,3,5-trimethoxybenzene ( $4 \mathrm{mg}, 0.025 \mathrm{mmol}$ ). The tube was recapped, pierced with a needle and reconnected to the Schlenk line via a rubber hose, evacuated and backfilled with nitrogen (the evacuation/backfill process was repeated a total of three times). THF (anhydrous, $500 \mu \mathrm{L}$ ) was added via syringe. The reaction mixture was allowed to stir at room temperature, or $60{ }^{\circ} \mathrm{C}$ (by immersing the reaction tube in a preheated oil bath) for $\sim 20 \mathrm{~h}$ (entries 1-6) or for $1 \mathrm{~h}$ (entries 7-14). At this time, the reaction vessel (if heated) was then removed from the oil bath and the reaction mixture was allowed to cool to room temperature. At room temperature, the reaction tube was opened to air, and the solution was transferred to a $20 \mathrm{~mL}$ scintillation vial and the original tube was washed with THF (stabilized, $1.5 \mathrm{~mL}$ ). The solution was concentrated in vacuo with the assistance of a rotary evaporator. The resulting residue was dissolved in $\mathrm{CDCl}_{3}(750 \mu \mathrm{L})$ and transferred to an NMR tube. The yield was determined by quantitative ${ }^{1} \mathrm{H}$ NMR spectroscopic analysis by integrating against 1,3,5-trimethoxybenzene.

\section{Synthesis of Oxidative Addition Complexes and Other Pd Complexes from P3}

\section{General Procedure A: Palladacycle, Ligand, and Aryl Halide all added at once}

An oven-dried $24 \mathrm{~mL}$ reaction tube was equipped, while still hot, with a magnetic stir bar and septum cap. The tube was allowed to cool to room temperature under vacuum by piercing the septum with a needle connected to a dual manifold by a rubber hose. At this point, the cap was removed and the tube was charged with palladacycle $\mathbf{P 3}(0.500 \mathrm{mmol}, 1.0$ equiv), ligand $(0.525$ mmol, 1.05 equiv), and aryl halide, if solid ( $0.600 \mathrm{mmol}, 1.2$ equiv). The tube was recapped, pierced with a needle and reconnected to the Schlenk line by a rubber hose, evacuated and backfilled with nitrogen (the evacuation/backfill process was repeated a total of three times). Under nitrogen, the aryl halide, if liquid $(0.600 \mathrm{mmol}, 1.2$ equiv) was added via syringe followed by $n$-hexane $(5.0 \mathrm{~mL}$ ). The tube was then placed in a pre-heated oil bath (bath temperature $=60$ ${ }^{\circ} \mathrm{C}$ ). The reaction mixture was allowed to stir at $60{ }^{\circ} \mathrm{C}$ for $2 \mathrm{~h}$. Over the course of the reaction, the mixture became homogeneous and subsequently the desired OAC precipitated. After $2 \mathrm{~h}$, the reaction vessel was removed from the oil bath and the mixture was allowed to cool to room temperature. The reaction tube was opened to air and the reaction mixture was diluted with pentane $(10 \mathrm{~mL})$ to assist with precipitation. The precipitate was collected on a fritted funnel and washed with pentane $(15 \mathrm{~mL})$ and dried in vacuo on a Schlenk line overnight $(\sim 16 \mathrm{~h})$ to afford the desired complex. 


\section{General Procedure B: Preformation of $\mathrm{Pd}^{0}$ Complexes $(\mathrm{L} \cdot \mathrm{Pd})_{2}(\operatorname{cod})$ Prior to Aryl Halide Addition}

An oven-dried $24 \mathrm{~mL}$ reaction tube was equipped, while still hot, with a magnetic stir bar and septum cap. The tube was allowed to cool to room temperature under vacuum by piercing the septum with a needle connected to a dual manifold by a rubber hose. At this point, the cap was removed and the tube was charged with palladacycle P3 $(0.600 \mathrm{mmol}, 1.20$ equiv) and ligand $(0.700-0.720 \mathrm{mmol}, 1.40-1.44$ equiv $)$. The tube was recapped, pierced with a needle and reconnected to the Schlenk line by a rubber hose, evacuated and backfilled with argon (the evacuation/backfill process was repeated a total of three times). Under argon, 2-MeTHF (2.5 mL) was added via syringe. The tube was then placed in a pre-heated oil bath (bath temperature $=60$ ${ }^{\circ} \mathrm{C}$ ). The reaction mixture was allowed to stir at $60^{\circ} \mathrm{C}$ for $30 \mathrm{~min}$. Over the course of the reaction, the mixture became homogeneous and subsequently the corresponding $(\mathrm{L} \cdot \mathrm{Pd})_{2}(\mathrm{cod})$ complex precipitated. After $30 \mathrm{~min}$, the reaction tube was removed from the oil bath and the mixture was allowed to cool to room temperature under argon for $10 \mathrm{~min}$. At this point, the cap was quickly removed and the aryl halide $(0.500 \mathrm{mmol}, 1.0$ equiv $)$ was added as a solid. The tube was recapped and the reaction mixture was further charged with THF (anhydrous, $5.0 \mathrm{~mL}$ ) via syringe by piercing the septum. The reaction mixture was allowed to stir at room temperature under argon for $1 \mathrm{~h}$. After $1 \mathrm{~h}$, the tube was opened to air, and the reaction mixture was filtered through a $\sim 1 \mathrm{~cm}$ plug of Celite ${ }^{\circledR}$ into a $50 \mathrm{~mL}$ round-bottom flask. The Celite ${ }^{\circledR}$ plug was further washed with THF (stabilized, $10 \mathrm{~mL}$ ). The combined filtrate solution was concentrated in vacuo with the assistance of a rotary evaporator. The resulting residue was dissolved in THF (stabilized, $5 \mathrm{~mL}$ ) and pipetted into a $100 \mathrm{~mL}$ beaker containing pentane $(50 \mathrm{~mL})$ to form a precipitate. The precipitate was collected on a fritted funnel and washed with pentane $(25 \mathrm{~mL})$ and dried in vacuo on a Schlenk line overnight $(\sim 16 \mathrm{~h})$ to afford the desired complex.

\section{General Procedure C: Synthesis of $\mathrm{Pd}^{0}$ Complexes $(\mathrm{L} \cdot \mathrm{Pd})_{2}(\operatorname{cod})$}

Note: this entire operation can be performed in a glovebox if it is equipped with a heating mantle. An oven-dried $24 \mathrm{~mL}$ reaction tube was equipped, while still hot, with a magnetic stir bar and solid cap. The tube was charged with palladacycle $\mathbf{P 3}(0.500 \mathrm{mmol}, 1.0$ equiv) and ligand $(0.525 \mathrm{mmol}$, 1.05 equiv). The tube was brought into a nitrogen-filled glovebox and cyclohexane $(5.0 \mathrm{~mL}) \mathrm{was}$ added via syringe. The tube was capped and the cap was sealed with electrical tape. The tube was removed from the glovebox and placed in a pre-heated oil bath (bath temperature $=60{ }^{\circ} \mathrm{C}$ ). The reaction mixture was allowed to stir at $60{ }^{\circ} \mathrm{C}$ for $2 \mathrm{~h}$. Over the course of the reaction, the mixture became homogeneous and subsequently the corresponding $(\mathrm{L} \cdot \mathrm{Pd})_{2}(\mathrm{cod})$ complex precipitated. After $2 \mathrm{~h}$, the reaction tube was removed from the oil bath and the mixture was allowed to cool to room temperature. At room temperature, the tube was returned to the glovebox. The cap was removed and the reaction mixture was diluted with pentane $(5 \mathrm{~mL})$. The precipitate was collected on a fritted funnel and washed with pentane $(15 \mathrm{~mL})$ and dried in vacuo in the glovebox for $1 \mathrm{~h}$ to obtain the desired complex. 


\section{(t-BuXPhos)Pd[(4-CO2TMSE)Ph]Br (1)}

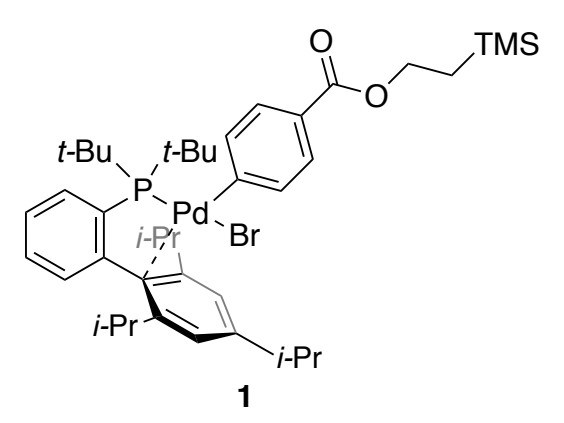

Compound 1 was prepared according to the General Procedure A using P3 (173 mg, $0.500 \mathrm{mmol}, 1$ equiv), $t$ BuXPhos (223 mg, $0.525 \mathrm{mmol}, 1.05$ equiv), 2(trimethylsilyl)ethyl 4-bromobenzoate $(181 \mathrm{mg}, 0.600$ mmol, 1.2 equiv), and $n$-hexane $(5.0 \mathrm{~mL})$. The precipitate was collected on a fritted funnel to yield $\mathbf{1}(376 \mathrm{mg}, 90 \%)$ as a pale-yellow powder.

Using $\mathbf{P 3}$ aged 1 year in air at room temperature, 1 was obtained as a pale-yellow powder (332 $\mathrm{mg}, 80 \%)$.

${ }^{1} \mathbf{H}$ NMR $\left(600 \mathrm{MHz}, \mathrm{CD}_{2} \mathrm{Cl}_{2}\right) \delta 8.01-7.96(\mathrm{~m}, 1 \mathrm{H}), 7.43(\mathrm{~d}, J=8.3 \mathrm{~Hz}, 2 \mathrm{H}), 7.42-7.39(\mathrm{~m}, 2 \mathrm{H})$, $7.25(\mathrm{~d}, J=6.8 \mathrm{~Hz}, 2 \mathrm{H}), 7.10(\mathrm{~s}, 2 \mathrm{H}), 6.79-6.72(\mathrm{~m}, 1 \mathrm{H}), 4.34-4.29(\mathrm{~m}, 2 \mathrm{H}), 3.07$ (hept, $J=6.9$ $\mathrm{Hz}, 1 \mathrm{H}$ ), 2.54 (hept, $J=6.8 \mathrm{~Hz}, 2 \mathrm{H}), 1.58$ (d, $J=6.8 \mathrm{~Hz}, 6 \mathrm{H}), 1.39$ (s, 9H), 1.38 (app s, 6H), 1.37 (s, 9H), $1.10-1.05(\mathrm{~m}, 2 \mathrm{H}), 0.90(\mathrm{~d}, J=6.7 \mathrm{~Hz}, 6 \mathrm{H}), 0.06(\mathrm{~s}, 9 \mathrm{H})$.

${ }^{13}$ C NMR $\left(151 \mathrm{MHz}, \mathrm{CD}_{2} \mathrm{Cl}_{2}\right) \delta 167.5,157.8,152.8,147.5,147.3,143.0,142.9,139.5,139.4$, $136.4,136.2,135.8,134.9,134.9,130.6,130.5,126.2,126.2,126.1,125.8,125.3,125.1,125.1$, $62.9,40.0,39.9,34.8,31.8,31.8,31.7,25.6,24.9,24.8,17.7,-1.4$.

${ }^{31} \mathbf{P}$ NMR $\left(203 \mathrm{MHz}, \mathrm{CD}_{2} \mathrm{Cl}_{2}\right) \delta 51.44$.

M.P. $185-187^{\circ} \mathrm{C}($ dec. $)$

IR (neat): 2958, 2920, 2899, 2861, 1703, 1604, 1571, 1476, 1462, 1374, 1272, 1263, 1246, 1170 , $1106,1047,1033,1007,964,931,874,848,837,784,749,732,694,661,600 \mathrm{~cm}^{-1}$

EA Calcd. for $\mathrm{C}_{41} \mathrm{H}_{62} \mathrm{BrO}_{2} \mathrm{PPdSi}$ : C, 59.17; H, 7.51. Found: C, 59.22; H, 7.58.

\section{(XPhos)Pd[(4-CO 2 TMSE)Ph]Br (2)}

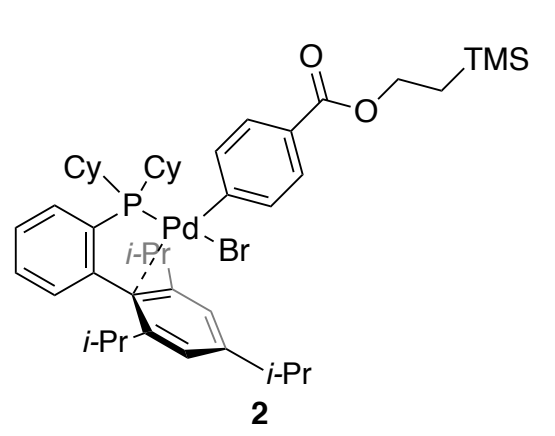

Compound $\mathbf{2}$ was prepared according to a modified General Procedure A using P3 (173 mg, $0.500 \mathrm{mmol}, 1$ equiv), XPhos (250 mg, 0.525 mmol, 1.05 equiv), 2(trimethylsilyl)ethyl 4-bromobenzoate (181 $\mathrm{mg}, 0.600$ mmol, 1.2 equiv), and $n$-hexane $(5.0 \mathrm{~mL})$. After $2 \mathrm{~h}$, no precipitate was observed. Therefore, the reaction mixture was allowed to cool to room temperature and transferred to a $25 \mathrm{~mL}$ round bottom flask with the assistance of pentane $(5.0 \mathrm{~mL})$. The combined solution was concentrated in vacuo with the assistance of a rotary evaporator to yield an orangecolored viscous oil. The oil was layered with pentane $(2.0 \mathrm{~mL})$ and triturated with the assistance of sonication to form a homogenous solution. The flask was gently scratched with a glass stir rod, sealed with a rubber septum, and placed in a freezer at $-25{ }^{\circ} \mathrm{C}$ for $\sim 22 \mathrm{~h}$. Over this period, a crystalline precipitate formed. The mixture is triturated with the assistance of sonication for $\sim 1$ min and returned to the $-25^{\circ} \mathrm{C}$ freezer for $1 \mathrm{~h}$. Over this period, a fine white precipitate had formed. The precipitate was collected on a fritted funnel and washed with cold pentane $\left(\sim-25^{\circ} \mathrm{C}, 10 \mathrm{~mL}\right)$ and dried in vacuo on the Schlenk line overnight $(\sim 16 \mathrm{~h})$ to yield $2(299 \mathrm{mg}, 68 \%)$ as a white powder. 
Note: Some additional peaks between 6.75 and $7.50 \mathrm{ppm}$ were observed by ${ }^{1} \mathrm{H}$ NMR spectroscopy in more concentrated samples of 2 . Thus, a more dilute ${ }^{1} \mathrm{H}$ NMR spectrum is also provided $(\sim 10$ $\mathrm{mg} / \mathrm{mL})$.

${ }^{1} \mathbf{H}$ NMR $\left(600 \mathrm{MHz}, \mathrm{CD}_{2} \mathrm{Cl}_{2}\right) \delta 7.72-7.68(\mathrm{~m}, 1 \mathrm{H}), 7.56(\mathrm{~d}, J=8.1 \mathrm{~Hz}, 2 \mathrm{H}), 7.47-7.43(\mathrm{~m}$, $2 \mathrm{H}), 7.19(\mathrm{dd}, J=8.4,1.9 \mathrm{~Hz}, 2 \mathrm{H}), 7.14(\mathrm{~s}, 2 \mathrm{H}), 6.84(\mathrm{dt}, J=5.2,3.2 \mathrm{~Hz}, 1 \mathrm{H}), 4.37-4.33$ (m, 2H), 3.10 (hept, $J=6.9 \mathrm{~Hz}, 1 \mathrm{H}$ ), 2.47 (hept, $J=6.8 \mathrm{~Hz}, 2 \mathrm{H}), 2.23$ (dtd, $J=12.7,9.8,2.6 \mathrm{~Hz}$, $2 \mathrm{H}), 1.94(\mathrm{~m}, 2 \mathrm{H}), 1.80(\mathrm{~d}, J=12.4 \mathrm{~Hz}, 2 \mathrm{H}), 1.76-1.62(\mathrm{~m}, 8 \mathrm{H}), 1.60(\mathrm{~d}, J=6.8 \mathrm{~Hz}, 6 \mathrm{H}), 1.39$ $(\mathrm{d}, J=7.0 \mathrm{~Hz}, 6 \mathrm{H}), 1.30-1.03(\mathrm{~m}, 10 \mathrm{H}), 0.90(\mathrm{~d}, J=6.7 \mathrm{~Hz}, 6 \mathrm{H}), 0.66(\mathrm{qt}, J=12.9,3.6 \mathrm{~Hz}$, $2 \mathrm{H}), 0.07(\mathrm{~s}, 9 \mathrm{H})$.

${ }^{13}$ C NMR $\left(151 \mathrm{MHz}, \mathrm{CD}_{2} \mathrm{Cl}_{2}\right) \delta 167.5,156.7,150.3,147.6,147.5,145.8,137.4,137.4,134.5$, $134.3,133.9,133.8,132.4,131.1,131.0,127.3,127.3,126.4,125.6,125.6,125.1,63.0,35.7$, $35.6,34.7,31.9,28.8,28.1,27.9,27.8,27.6,27.5,26.3,25.5,24.8,24.6,24.3,17.6,-1.4$.

${ }^{31} \mathbf{P}$ NMR $\left(203 \mathrm{MHz}, \mathrm{CD}_{2} \mathrm{Cl}_{2}\right) \delta 27.46$.

M.P. $199-201{ }^{\circ} \mathrm{C}$ (dec.)

IR (neat): 3060, 2953, 2920, 2854, 1708, 1606, 1571, 1464, 1450, 1443, 1419, 1381, 1357, 1317 , $1301,1267,1256,1201,1170,1102,1083,1050,1033,1007,998,964,936,917,877,834,780$, $754,735,713,692,666,649,623 \mathrm{~cm}^{-1}$

EA Calcd. for $\mathrm{C}_{45} \mathrm{H}_{66} \mathrm{BrO}_{2} \mathrm{PPdSi}$ : C, 61.11; H, 7.52. Found: C, 60.83; H, 7.55.

(t-BuBrettPhos)Pd[(4-CO2TMSE)Ph]Br (3)
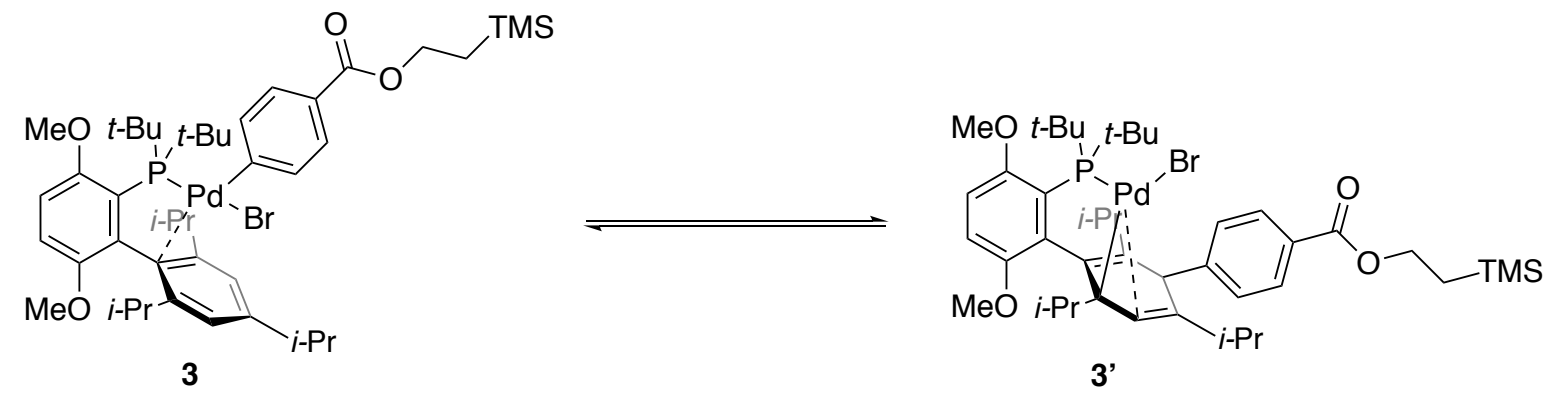

Compound 3 was prepared according to a modified General Procedure A using P3 (173 mg, 0.500 mmol, 1 equiv), $t$-BuBrettPhos (255 mg, $0.525 \mathrm{mmol}, 1.05$ equiv), 2-(trimethylsilyl)ethyl 4bromobenzoate $(181 \mathrm{mg}, 0.600 \mathrm{mmol}, 1.2$ equiv), and cyclohexane $(5.0 \mathrm{~mL})$. The reaction was allowed to stir at $80{ }^{\circ} \mathrm{C}$ for $2 \mathrm{~h}$. The precipitate was collected on a fritted funnel to yield 3 (334 $\mathrm{mg}, 74 \%$ ) as a pale-yellow powder. The ${ }^{1} \mathrm{H},{ }^{13} \mathrm{C}$, and ${ }^{31} \mathrm{P}$ NMR and IR spectroscopic data matched that reported in the literature. ${ }^{4}$

${ }^{1} \mathbf{H}$ NMR $\left(600 \mathrm{MHz}, \mathrm{CD}_{2} \mathrm{Cl}_{2}\right) \delta 7.38(\mathrm{~d}, J=8.2 \mathrm{~Hz}, 2 \mathrm{H}), 7.24(\mathrm{~d}, J=6.9 \mathrm{~Hz}, 2 \mathrm{H}), 7.05(\mathrm{~s}, 2 \mathrm{H})$, $6.94(\mathrm{dd}, J=8.9,2.6 \mathrm{~Hz}, 1 \mathrm{H}), 6.87(\mathrm{~d}, J=8.9 \mathrm{~Hz}, 1 \mathrm{H}), 4.34-4.28(\mathrm{~m}, 2 \mathrm{H}), 3.79(\mathrm{~s}, 3 \mathrm{H}), 3.33$ (s, 3H), 3.04 (hept, $J=6.9 \mathrm{~Hz}, 1 \mathrm{H}), 2.60$ (hept, $J=6.8 \mathrm{~Hz}, 2 \mathrm{H}), 1.60$ (d, $J=6.8 \mathrm{~Hz}, 6 \mathrm{H}), 1.40$ (s, $9 \mathrm{H}), 1.37(\mathrm{~s}, 9 \mathrm{H}), 1.36(\mathrm{~d}, J=7.1 \mathrm{~Hz}, 6 \mathrm{H}), 1.10-1.05(\mathrm{~m}, 2 \mathrm{H}), 0.82(\mathrm{~d}, J=6.7 \mathrm{~Hz}, 6 \mathrm{H}), 0.06$ (s, 9H).

${ }^{13}$ C NMR $\left(151 \mathrm{MHz}, \mathrm{CD}_{2} \mathrm{Cl}_{2}\right) \delta 167.8,154.8,154.4,154.3,152.2,152.1,146.4,146.3,140.4$, $140.4,127.8,127.6,125.4,125.2$, 125.0, 116.7, 116.6, 113.8, 110.9, 110.9, 62.7, 54.7, 41.6, 41.5, $34.9,32.7,32.7,31.7,25.9,24.9,24.6,17.7,-1.4$.

${ }^{31} \mathbf{P}$ NMR $\left(203 \mathrm{MHz}, \mathrm{CD}_{2} \mathrm{Cl}_{2}\right) \delta 82.86,72.00$. 
M.P. $188-190{ }^{\circ} \mathrm{C}($ dec.)

IR (neat): 2953, 2899, 2863, 2828, 1694, 1606, 1571, 1455, 1419, 1381, 1357, 1302, 1260, 1249 , $1173,1159,1104,1092,1066,1050,1007,955,938,865,839,815,754,716,699 \mathrm{~cm}^{-1}$

(RuPhos)Pd[(4-CO2TMSE)Ph]Br (4)

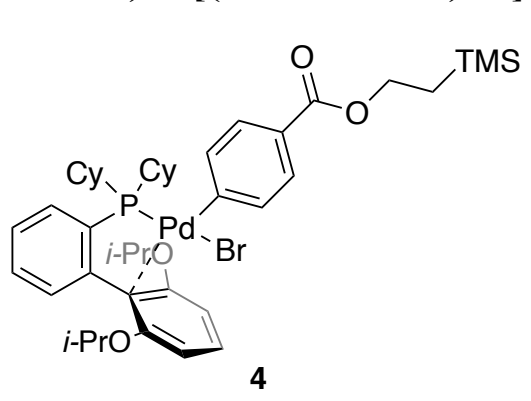

Compound 4 was prepared according to the General Procedure A using P3 (173 mg, $0.500 \mathrm{mmol}, 1$ equiv), RuPhos (245 mg, 0.525 mmol, 1.05 equiv), 2(trimethylsilyl)ethyl 4-bromobenzoate (181 mg, 0.600 mmol, 1.2 equiv), and $n$-hexane $(5.0 \mathrm{~mL})$. The precipitate was collected on a fritted funnel to yield $4(354 \mathrm{mg}, 81 \%)$ as a white powder.

${ }^{1} \mathbf{H}$ NMR $\left(500 \mathrm{MHz}, \mathrm{CD}_{2} \mathrm{Cl}_{2}\right) \delta 7.62(\mathrm{q}, J=7.8,7.3 \mathrm{~Hz}, 2 \mathrm{H})$, $7.54(\mathrm{~d}, J=7.7 \mathrm{~Hz}, 2 \mathrm{H}), 7.45(\mathrm{tt}, J=7.5,1.5 \mathrm{~Hz}, 1 \mathrm{H}), 7.39(\mathrm{tt}, J=7.3,1.4 \mathrm{~Hz}, 1 \mathrm{H}), 7.23(\mathrm{dd}, J=$ $8.5,2.0 \mathrm{~Hz}, 2 \mathrm{H}$ ), 6.87 (ddd, $J=7.7,3.2,1.4 \mathrm{~Hz}, 1 \mathrm{H}), 6.66$ (d, $J=8.4 \mathrm{~Hz}, 2 \mathrm{H}), 4.62$ (hept, $J=6.1$ $\mathrm{Hz}, 2 \mathrm{H}), 4.37-4.31(\mathrm{~m}, 2 \mathrm{H}), 2.13(\mathrm{dtd}, J=12.5,9.6,2.7 \mathrm{~Hz}, 2 \mathrm{H}), 1.76(\mathrm{~d}, J=11.8 \mathrm{~Hz}, 6 \mathrm{H}), 1.70$ $-1.54(\mathrm{~m}, 6 \mathrm{H}), 1.39(\mathrm{~d}, J=6.0 \mathrm{~Hz}, 6 \mathrm{H}), 1.27-1.05(\mathrm{~m}, 9 \mathrm{H}), 1.02(\mathrm{~d}, J=6.0 \mathrm{~Hz}, 6 \mathrm{H}), 0.78(\mathrm{qt}, J$ $=12.8,3.9 \mathrm{~Hz}, 2 \mathrm{H}), 0.07(\mathrm{~s}, 9 \mathrm{H})$.

${ }^{13} \mathrm{C}$ NMR $\left(126 \mathrm{MHz}, \mathrm{CD}_{2} \mathrm{Cl}_{2}\right) \delta 167.6,159.6,147.4,145.1,144.9,138.0,138.0,135.1,133.4$, $133.1,133.0,132.9,131.4,131.1,131.0,127.1,127.0,126.8,126.8,126.2,111.7,111.6,107.9$, 71.5, 62.9, 34.3, 34.1, 28.6, 28.1, 28.1, 27.5, 27.4, 27.2, 27.1, 26.4, 22.3, 21.7, 17.7, -1.4.

${ }^{31} \mathbf{P}$ NMR $\left(203 \mathrm{MHz}, \mathrm{CD}_{2} \mathrm{Cl}_{2}\right) \delta 31.51$.

M.P. $201-203{ }^{\circ} \mathrm{C}$ (dec.)

IR (neat): 2972, 2932, 2849, 1710, 1587, 1573, 1447, 1384, 1372, 1270, 1246, 1168, 1111, 1095 , 1085, 1066, 1012, 943, 865, 848, 837, 766, 754, 735, $692 \mathrm{~cm}^{-1}$

EA Calcd. for $\mathrm{C}_{42} \mathrm{H}_{60} \mathrm{BrO}_{4} \mathrm{PPdSi}$ : C, 57.70; H, 6.92. Found: C, 57.82; H, 7.10.

(AdCyBrettPhos)Pd[(4-CO2TMSE)Ph]Br (5)

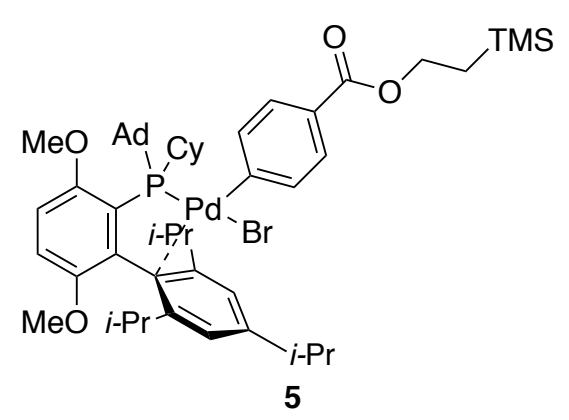

Compound 5 was prepared according to the General Procedure A using $\mathbf{P 3}$ (173 mg, $0.500 \mathrm{mmol}, 1$ equiv), AdCyBrettPhos (309 mg, $0.525 \mathrm{mmol}, 1.05$ equiv), 2(trimethylsilyl)ethyl 4-bromobenzoate (181 mg, 0.600 mmol, 1.2 equiv), and $n$-hexane $(5.0 \mathrm{~mL})$. The precipitate was collected on a fritted funnel to yield 5 (461 $\mathrm{mg}, 92 \%)$ as a pale-yellow powder.

${ }^{1} \mathbf{H}$ NMR $\left(600 \mathrm{MHz}, \mathrm{CD}_{2} \mathrm{Cl}_{2}\right) \delta 7.51-7.48(\mathrm{~m}, 1 \mathrm{H}), 7.44(\mathrm{~d}, J=7.7 \mathrm{~Hz}, 1 \mathrm{H}), 7.39(\mathrm{~d}, J=8.5$ $\mathrm{Hz}, 1 \mathrm{H}), 7.24(\mathrm{~d}, J=8.3 \mathrm{~Hz}, 1 \mathrm{H}), 7.09$ (d, $J=1.7 \mathrm{~Hz}, 1 \mathrm{H}), 7.06(\mathrm{~d}, J=1.8 \mathrm{~Hz}, 1 \mathrm{H}), 6.92(\mathrm{dd}, J=$ 9.0, 2.7 Hz, 1H), $6.86(\mathrm{~d}, J=8.9 \mathrm{~Hz}, 1 \mathrm{H}), 4.37-4.30(\mathrm{~m}, 2 \mathrm{H}), 3.82(\mathrm{~s}, 3 \mathrm{H}), 3.35(\mathrm{~s}, 3 \mathrm{H}), 3.05$ (hept, $J=6.9 \mathrm{~Hz}, 1 \mathrm{H}), 2.87$ (q, $J=11.7 \mathrm{~Hz}, 1 \mathrm{H}$ ), 2.64 (hept, $J=6.7 \mathrm{~Hz}, 1 \mathrm{H}$ ), 2.55 (hept, $J=6.7$ $\mathrm{Hz}, 1 \mathrm{H}), 2.14(\mathrm{~d}, J=11.2 \mathrm{~Hz}, 3 \mathrm{H}), 2.00-1.86(\mathrm{~m}, 8 \mathrm{H}), 1.81-1.74(\mathrm{~m}, 2 \mathrm{H}), 1.69(\mathrm{q}, J=12.8$ $\mathrm{Hz}, 8 \mathrm{H}), 1.60(\mathrm{dd}, J=13.0,6.8 \mathrm{~Hz}, 7 \mathrm{H}), 1.37(\mathrm{dd}, J=6.9,4.3 \mathrm{~Hz}, 6 \mathrm{H}), 1.33-1.24(\mathrm{~m}, 1 \mathrm{H})$, 
$1.23-1.13(\mathrm{~m}, 2 \mathrm{H}), 1.12-1.06(\mathrm{~m}, 2 \mathrm{H}), 0.97(\mathrm{~d}, J=6.7 \mathrm{~Hz}, 3 \mathrm{H}), 0.66$ (d, $J=6.7 \mathrm{~Hz}, 3 \mathrm{H}), 0.07$ (s, 9H).

${ }^{13}$ C NMR $\left(151 \mathrm{MHz}, \mathrm{CD}_{2} \mathrm{Cl}_{2}\right) \delta 167.7,157.8,155.1,155.0,154.5,153.8,152.4,152.3,147.9$, 141.3, 138.3, 138.2, 137.3, 126.6, 125.7, 125.5, 125.5, 125.4, 125.0, 124.8, 116.7, 116.7, 113.7, 113.7, 110.8, 110.8, 62.8, 54.8, 54.8, 44.1, 44.0, 41.3, 37.0, 36.9, 36.7, 34.8, 32.1, 31.5, 31.1, $30.4,29.9,29.8,28.5,28.4,28.1,28.0,26.7,26.6,25.0,24.9,24.8,24.6,24.5,17.6,-1.4$.

${ }^{31} \mathbf{P}$ NMR $\left(162 \mathrm{MHz}, \mathrm{CD}_{2} \mathrm{Cl}_{2}\right) \delta 49.86$.

M.P. $208-211^{\circ} \mathrm{C}$ (dec.)

IR (neat): 3060, 2951, 2923, 2904, 2847, 1710, 1606, 1573, 1549, 1457, 1421, 1383, 1357, 1341, $1301,1258,1170,1102,1085,1052,1009,964,936,886,836,818,784,754,730,713,694,661$, $649,616 \mathrm{~cm}^{-1}$

EA Calcd. for $\mathrm{C}_{51} \mathrm{H}_{74} \mathrm{BrO}_{4} \mathrm{PPdSi}$ : C, 61.47; H, 7.49. Found: C, 61.52; H, 7.59.

(GPhos)Pd[(4-CO 2 TMSE)Ph]Br (6)

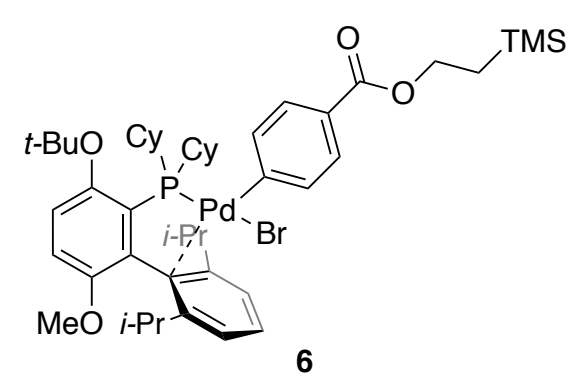

Compound 6 was prepared according to the General Procedure A using $\mathbf{P 3}$ (173 mg, $0.500 \mathrm{mmol}, 1$ equiv), GPhos (282 mg, $0.525 \mathrm{mmol}, 1.05$ equiv), 2(trimethylsilyl)ethyl 4-bromobenzoate $(181 \mathrm{mg}, 0.600$ mmol, 1.2 equiv), and $n$-hexane $(5.0 \mathrm{~mL})$. The precipitate was collected on a fritted funnel to yield 6 (433 $\mathrm{mg}, 92 \%)$ as an off-white powder. The ${ }^{1} \mathrm{H},{ }^{13} \mathrm{C}$, and ${ }^{31} \mathrm{P}$ NMR and IR spectroscopic data matched that reported in the literature. ${ }^{10}$

${ }^{1} \mathbf{H}$ NMR $\left(600 \mathrm{MHz}, \mathrm{CDCl}_{3}\right) \delta 7.80(\mathrm{t}, J=7.8 \mathrm{~Hz}, 1 \mathrm{H}), 7.52(\mathrm{~d}, J=8.2 \mathrm{~Hz}, 2 \mathrm{H}), 7.26-7.21(\mathrm{~m}$, $4 \mathrm{H}), 7.09(\mathrm{dd}, J=9.1,3.1 \mathrm{~Hz}, 1 \mathrm{H}), 6.79(\mathrm{~d}, J=9.1 \mathrm{~Hz}, 1 \mathrm{H}), 4.36-4.30(\mathrm{~m}, 2 \mathrm{H}), 3.37(\mathrm{~s}, 3 \mathrm{H})$, $3.02-2.93(\mathrm{~m}, 2 \mathrm{H}), 2.51$ (hept, $J=6.7 \mathrm{~Hz}, 2 \mathrm{H}), 1.85-1.65(\mathrm{~m}, 12 \mathrm{H}), 1.63(\mathrm{~s}, 9 \mathrm{H}), 1.59$ (d, $J=$ $6.8 \mathrm{~Hz}, 6 \mathrm{H}), 1.21-1.12(\mathrm{~m}, 6 \mathrm{H}), 1.09-1.04(\mathrm{~m}, 2 \mathrm{H}), 0.98-0.88(\mathrm{~m}, 2 \mathrm{H}), 0.84(\mathrm{~d}, J=6.7 \mathrm{~Hz}$, $6 \mathrm{H}), 0.05$ (s, 9H).

${ }^{13}$ C NMR $\left(151 \mathrm{MHz}, \mathrm{CDCl}_{3}\right) \delta 167.9,152.1,151.9,151.9,150.9,150.8,145.7,145.7,138.5$, $138.5,137.8,137.7,134.2,126.6,126.2,125.9,125.7,125.5,120.3,120.3,115.7,115.7,113.0$, 113.0, 79.7, 62.6, 54.6, 36.3, 36.1, 31.4, 29.6, 29.3, 29.3, 27.8, 27.7, 27.7, 27.6, 26.0, 25.5, 24.7, 17.4, -1.3 .

${ }^{31} \mathbf{P}$ NMR $\left(203 \mathrm{MHz}, \mathrm{CD}_{2} \mathrm{Cl}_{2}\right) \delta 36.90$.

M.P. $185-186^{\circ} \mathrm{C}($ dec. $)$

IR (neat): 2958, 2927, 2849, 1706, 1573, 1450, 1421, 1398, 1379, 1265, 1208, 1156, 1111, 1099, $1087,1052,1009,936,860,848,832,813,792,749,735,720,692,619 \mathrm{~cm}^{-1}$ 


\section{(AlPhos)Pd[(4-CF 3$) P h] B r(7)$}

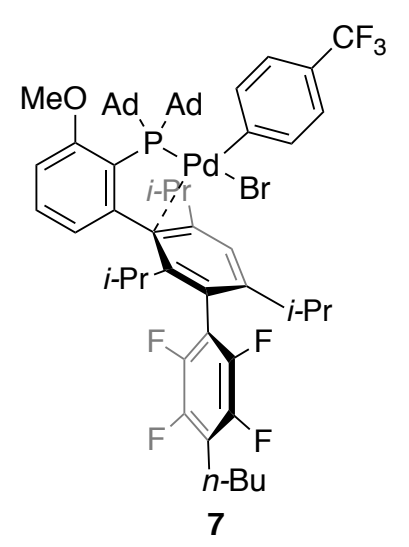

Compound 7 was prepared according to the General Procedure A using P3 (173 mg, $0.500 \mathrm{mmol}, 1$ equiv), AlPhos (428 mg, 0.525 mmol, 1.05 equiv), 4-Bromobenzotrifluoride ( $84 \mu \mathrm{L}, 0.600 \mathrm{mmol}, 1.2$ equiv), and $n$-hexane $(5.0 \mathrm{~mL})$. The precipitate was collected on a fritted funnel to yield 7 (308 mg, 54\%) as a yellow powder. The ${ }^{1} \mathrm{H}$, ${ }^{13} \mathrm{C},{ }^{19} \mathrm{~F}$, and ${ }^{31} \mathrm{P}$ NMR and IR spectroscopic data matched that reported in the literature. ${ }^{4}$

${ }^{1}$ H NMR $\left(600 \mathrm{MHz}, \mathrm{CD}_{2} \mathrm{Cl}_{2}\right) \delta 7.46-7.35(\mathrm{~m}, 3 \mathrm{H}), 7.32(\mathrm{~s}, 1 \mathrm{H})$, $7.08(\mathrm{t}, J=6.8 \mathrm{~Hz}, 2 \mathrm{H}), 6.94(\mathrm{~d}, J=8.2 \mathrm{~Hz}, 1 \mathrm{H}), 6.31$ (dd, $J=7.8$, $2.7 \mathrm{~Hz}, 1 \mathrm{H}), 3.90$ (s, 3H), 3.24 (hept, $J=7.1 \mathrm{~Hz}, 1 \mathrm{H}), 2.82$ (t, $J=7.7$ $\mathrm{Hz}, 2 \mathrm{H}), 2.48-2.33$ (m, 8H), $2.13-2.08$ (m, 3H), 2.04 (s, 3H), 1.85 $-1.72(\mathrm{~m}, 12 \mathrm{H}), 1.71-1.59(\mathrm{~m}, 8 \mathrm{H}), 1.58-1.51(\mathrm{~m}, 3 \mathrm{H}), 1.43(\mathrm{dq}$, $J=14.8,7.4 \mathrm{~Hz}, 2 \mathrm{H}), 1.32(\mathrm{~d}, J=6.8 \mathrm{~Hz}, 3 \mathrm{H}), 1.26(\mathrm{~d}, J=7.1 \mathrm{~Hz}, 3 \mathrm{H}), 1.12(\mathrm{~d}, J=6.8 \mathrm{~Hz}, 3 \mathrm{H})$, 0.98 (t, $J=7.4 \mathrm{~Hz}, 3 \mathrm{H}), 0.84$ (dd, $J=7.1,2.0 \mathrm{~Hz}, 3 \mathrm{H}), 0.79$ (d, $J=6.6 \mathrm{~Hz}, 3 \mathrm{H})$.

${ }^{13}$ C NMR $\left(151 \mathrm{MHz}, \mathrm{CD}_{2} \mathrm{Cl}_{2}\right) \delta 161.4,161.4,157.0,153.0,151.0,150.1,150.0,142.3,142.3$, $141.1,138.5,132.1,132.0,128.5,128.5,128.1,128.0,126.9,126.3,126.1,124.9,124.7,124.5$, 124.4, 124.2, 123.5, 123.4, 121.8, 121.6, 121.2, 121.1, 121.0, 117.8, 117.6, 117.5, 109.8, 109.8, $48.1,48.0,47.0,46.9,42.9,42.9,40.5,36.8,36.5,33.5,31.8,31.5,31.5,30.1,30.0,29.8,29.8$, $26.4,25.4,24.9,24.4,24.0,23.1,22.8,22.3,22.3,14.0$.

${ }^{19} \mathbf{F}$ NMR $\left(565 \mathrm{MHz}, \mathrm{CD}_{2} \mathrm{Cl}_{2}\right) \delta-62.50,-132.55(\mathrm{dd}, J=23.3,12.5 \mathrm{~Hz}),-138.02(\mathrm{dd}, J=23.7$, $12.2 \mathrm{~Hz}),-146.16$ (dd, $J=23.4,12.4 \mathrm{~Hz}),-147.08$ (dd, $J=23.4,12.4 \mathrm{~Hz})$.

${ }^{31} \mathbf{P}$ NMR $\left(162 \mathrm{MHz}, \mathrm{CD}_{2} \mathrm{Cl}_{2}\right) \delta 65.50$.

M.P. $192-195{ }^{\circ} \mathrm{C}$ (dec.)

IR (neat): 2958, 2904, 2847, 1582, 1563, 1473, 1466, 1450, 1383, 1362, 1322, 1301, 1279, 1267 , $1154,1099,1064,1040,1005,976,915,827,808,803,765,742,720,635 \mathrm{~cm}^{-1}$

\section{(AlPhos)Pd[(4-CF 3$) P h] O T f(8)$}



Compound $\mathbf{8}$ was prepared according to a modified General Procedure A. An oven-dried $24 \mathrm{~mL}$ reaction tube was equipped, while still hot, with a magnetic stir bar and septum cap. The tube was allowed to cool to room temperature under vacuum by piercing the septum with a needle connected to a dual manifold by a rubber hose. The reaction tube was opened to air and charged with $\mathbf{P 3}$ (173 $\mathrm{mg}, 0.500 \mathrm{mmol}, 1$ equiv) and AlPhos (428 mg, $0.525 \mathrm{mmol}, 1.05$ equiv). The tube was brought into a $\mathrm{N}_{2}$-filled glovebox and charged with 4(trifluoromethyl)phenyl trifluoromethanesulfonate (294 mg, 1.000 mmol, 2.0 equiv), and cyclohexane $(10.0 \mathrm{~mL})$. The reaction tube was capped, removed from the glove box, and placed under $\mathrm{N}_{2}$ by piercing the septum with a needled connected to a dual manifold by a rubber hose. The reaction mixture was allowed to stir at $60{ }^{\circ} \mathrm{C}$ for $\sim 22 \mathrm{~h}$.

Over this period, an orange-colored precipitate formed. At this time, the reaction tube was removed from the oil bath and the reaction mixture was allowed to cool to room temperature. The tube was then opened to air and the reaction mixture was diluted with pentane $(10 \mathrm{~mL})$. The orange-colored precipitate was collected on a fritted funnel and washed with pentane $(15 \mathrm{~mL})$. By ${ }^{1} \mathrm{H}$ and ${ }^{19} \mathrm{~F}$ 
NMR spectroscopic analysis, the precipitate contained $\sim 10 \%$ unreacted triflate. The precipitate was transferred to a $50 \mathrm{~mL}$ round-bottom flask and suspended in pentane $(25 \mathrm{~mL})$. The suspension was triturated with the assistance of sonication and the resulting orange powder was collected on a fritted funnel and washed with pentane $(25 \mathrm{~mL})$ and dried in vacuo on the Schlenk line overnight $(\sim 16 \mathrm{~h})$ to yield $\mathbf{8}(340 \mathrm{mg}, 56 \%)$ as an orange powder.

Note: there are significant differences in chemical shifts of peaks in the ${ }^{1} \mathrm{H}$ NMR spectra between samples prepared in $\mathrm{CD}_{2} \mathrm{Cl}_{2}$ and $\mathrm{C}_{6} \mathrm{D}_{6}$. Thus, a copy of the ${ }^{1} \mathrm{H} \mathrm{NMR}$ spectrum in $\mathrm{C}_{6} \mathrm{D}_{6}$ is also provided, which matches that reported in the literature. ${ }^{4}$

${ }^{1} \mathbf{H}$ NMR $\left(500 \mathrm{MHz}, \mathrm{CD}_{2} \mathrm{Cl}_{2}\right) \delta 7.84-7.79(\mathrm{~m}, 1 \mathrm{H}), 7.60-7.54(\mathrm{~m}, 2 \mathrm{H}), 7.37(\mathrm{~s}, 1 \mathrm{H}), 7.32-$ $7.29(\mathrm{~m}, 1 \mathrm{H}), 7.27(\mathrm{~d}, J=8.4 \mathrm{~Hz}, 3 \mathrm{H}), 4.03(\mathrm{~s}, 3 \mathrm{H}), 2.84(\mathrm{t}, J=7.7 \mathrm{~Hz}, 2 \mathrm{H}), 2.72$ (dhept, $J=$ $14.0,6.9 \mathrm{~Hz}, 2 \mathrm{H}), 2.24-2.10(\mathrm{~m}, 7 \mathrm{H}), 2.02(\mathrm{~d}, J=28.6 \mathrm{~Hz}, 12 \mathrm{H}), 1.79-1.68(\mathrm{~m}, 15 \mathrm{H}), 1.68-$ $1.61(\mathrm{~m}, 2 \mathrm{H}), 1.41(\mathrm{~h}, J=7.4 \mathrm{~Hz}, 2 \mathrm{H}), 1.22(\mathrm{~d}, J=6.7 \mathrm{~Hz}, 3 \mathrm{H}), 1.03(\mathrm{dd}, J=10.1,6.9 \mathrm{~Hz}, 6 \mathrm{H})$, $0.96(\mathrm{t}, J=7.3 \mathrm{~Hz}, 3 \mathrm{H}), 0.82(\mathrm{~d}, J=6.9 \mathrm{~Hz}, 3 \mathrm{H}), 0.19(\mathrm{~d}, J=6.8 \mathrm{~Hz}, 3 \mathrm{H})$.

${ }^{13}$ C NMR $\left(126 \mathrm{MHz}, \mathrm{CD}_{2} \mathrm{Cl}_{2}\right) \delta 161.5,155.5(\mathrm{~d}, J=2.7 \mathrm{~Hz}), 154.4,148.9,146.9,146.6,146.5$ $(\mathrm{d}, J=16.5 \mathrm{~Hz}), 144.9$ (d, $J=14.6 \mathrm{~Hz}), 144.6,144.4(\mathrm{~d}, J=10.7 \mathrm{~Hz}), 142.9(\mathrm{dd}, J=33.3,15.1$ $\mathrm{Hz}), 139.9$ (t, $J=2.7 \mathrm{~Hz}), 135.3(\mathrm{~d}, J=2.1 \mathrm{~Hz}), 129.3$ (q, $J=32.4 \mathrm{~Hz}), 125.6,124.5$ (d, $J=9.3$ $\mathrm{Hz}), 124.1(\mathrm{q}, J=3.8 \mathrm{~Hz}), 123.9,123.7,123.5,123.4,123.3,122.8,113.2(\mathrm{t}, J=19.5 \mathrm{~Hz}), 112.9$ $(\mathrm{d}, J=3.9 \mathrm{~Hz}), 112.3(\mathrm{~d}, J=2.6 \mathrm{~Hz}), 55.4,48.4(\mathrm{dd}, J=24.1,10.2 \mathrm{~Hz}), 41.9(\mathrm{~d}, J=16.2 \mathrm{~Hz})$, 36.2 (dd, $J=7.8,1.7 \mathrm{~Hz}$ ), 33.1, 32.4, 31.9, 31.6, 29.5 (dd, $J=14.2,10.1 \mathrm{~Hz}), 26.2,24.3,24.1$ (d, $J=3.7 \mathrm{~Hz}), 23.2,22.7,22.4,21.5,21.2,13.8$.

${ }^{19}$ F NMR $\left(471 \mathrm{MHz}, \mathrm{CD}_{2} \mathrm{Cl}_{2}\right) \delta-61.66,-77.85,-134.83--135.21(\mathrm{~m}),-136.30--136.84(\mathrm{~m})$, $-141.21--141.65(\mathrm{~m}),-141.80--142.24(\mathrm{~m})$.

${ }^{31} \mathbf{P}$ NMR $\left(203 \mathrm{MHz}, \mathrm{CD}_{2} \mathrm{Cl}_{2}\right) \delta 118.87$.

M.P. $227-229^{\circ} \mathrm{C}$ (dec.)

IR (neat): 3079. 2972, 2930, 2908, 2854, 1585, 1563, 1478, 1466, 1393, 1381, 1369, 1324, 1301, 1263, 1222, 1192, 1161, 1149, 1121, 1099, 1071, 1028, 1005, 974, 938, 919, 896, 836, 820, 803, $770,751,723,675,635 \mathrm{~cm}^{-1}$

\section{(BrettPhos)Pd[(4-n-Butyl)Ph]Br (9)}

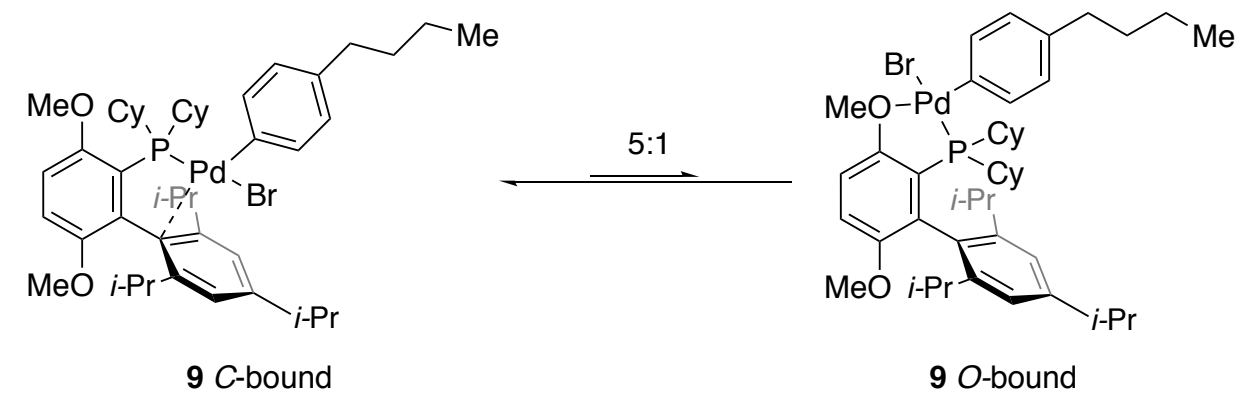

Compound 9 was prepared according to the General Procedure A using P3 (173 mg, $0.500 \mathrm{mmol}$, 1 equiv), BrettPhos ( $282 \mathrm{mg}, 0.525 \mathrm{mmol}, 1.05$ equiv), 4-bromo- $n$-butylbenzene (106 $\mu \mathrm{L}, 0.600$ mmol, 1.2 equiv), and $n$-hexane $(5.0 \mathrm{~mL})$. The precipitate was collected on a fritted funnel to yield $9(387 \mathrm{mg}, 90 \%)$ as a pale-yellow powder. Compound 9 was determined to be a 5:1 mixture of $C$ Bound to $O$-Bound isomer. The ${ }^{1} \mathrm{H},{ }^{13} \mathrm{C}$, and ${ }^{31} \mathrm{P}$ NMR and IR spectroscopic data matched that reported in the literature. ${ }^{16}$ 
${ }^{1} \mathrm{H}$ NMR $\left(500 \mathrm{MHz}, \mathrm{CD}_{2} \mathrm{Cl}_{2}\right.$ ) Complex spectrum, see below.

${ }^{13} \mathbf{C}$ NMR $\left(126 \mathrm{MHz}, \mathrm{CD}_{2} \mathrm{Cl}_{2}\right) \delta 157.0,156.9,156.9,155.3,155.3,154.3,154.2,152.4,152.3$, $152.1,149.4,147.3,138.6,138.4,138.2,138.2,137.7,137.7,137.6,133.5,133.5,133.4,133.4$, $131.1,130.9,127.2,127.2,127.0,127.0,125.3,125.1,124.6,121.9,119.1,118.9,118.0,118.0$, 113.7, 113.7, 111.7, 111.7, 111.4, 111.4, 62.2, 55.2, 55.1, 54.7, 36.8, 36.6, 35.4, 35.2, 35.1, 34.7, 34.6, 34.5, 32.0, 31.8, 31.3, 31.2, 31.1, 29.7, 29.7, 29.3, 29.3, 28.3, 28.2, 28.1, 28.1, 27.1, 27.0, $26.9,26.8,26.4,26.4,25.6,25.6,25.4,24.9,24.9,24.6,24.6,24.1,23.5,23.1,22.5,22.5,14.3$, $14.2,14.1$.

${ }^{31}$ P NMR $\left(203 \mathrm{MHz}, \mathrm{CD}_{2} \mathrm{Cl}_{2}\right) \delta 45.38,37.90$.

M.P. $202-204{ }^{\circ} \mathrm{C}(\mathrm{dec}$. $)$

IR (neat): 2958, 2918, 2849, 1604, 1575, 1476, 1455, 1424, 1379, 1357, 1298, 1256, 1185, 1173 , $1159,1121,1083,1054,1005,945,917,886,870,851,818,787,780,766,754,737,709,647$, $621 \mathrm{~cm}^{-1}$

\section{(CPhos)Pd[(4-OMe)Ph]Cl (10)}

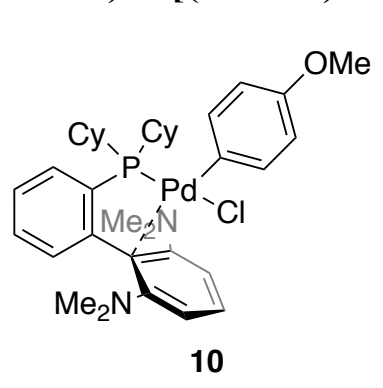

Compound $\mathbf{1 0}$ was prepared according to the General Procedure A using P3 (173 mg, $0.500 \mathrm{mmol}, 1$ equiv), CPhos (229 mg, 0.525 mmol, 1.05 equiv), 4-chloroanisole ( $75 \mu \mathrm{L}, 0.600 \mathrm{mmol}, 1.2$ equiv), and $n$-hexane $(5.0 \mathrm{~mL})$. The precipitate was collected on a fritted funnel to yield $10(296 \mathrm{mg}, 86 \%)$ as a pale-tan powder.

Note: Compound 10 contains $\sim 10 \% n$-hexane as determined by ${ }^{1} \mathrm{H}$ and ${ }^{13} \mathrm{C}$ NMR spectroscopy. The $n$-hexane could not be removed even after heating $\left(60^{\circ} \mathrm{C}\right)$ under high vacuum $(<50 \mathrm{mTorr})$ for $24 \mathrm{~h}$. The ppm by one proton in the ${ }^{1} \mathrm{H}$ NMR spectrum.

presence of this solvent causes over-integration of peaks at 0.9 and 1.2

${ }^{1} \mathbf{H}$ NMR $\left(500 \mathrm{MHz}, \mathrm{CD}_{2} \mathrm{Cl}_{2}\right) \delta 7.71(\mathrm{t}, J=7.3 \mathrm{~Hz}, 1 \mathrm{H}), 7.57(\mathrm{t}, J=8.1 \mathrm{~Hz}, 1 \mathrm{H}), 7.45(\mathrm{t}, J=7.5$ $\mathrm{Hz}, 1 \mathrm{H}), 7.36(\mathrm{t}, J=7.6 \mathrm{~Hz}, 1 \mathrm{H}), 7.11-7.06(\mathrm{~m}, 1 \mathrm{H}), 6.92(\mathrm{dd}, J=23.3,7.4 \mathrm{~Hz}, 4 \mathrm{H}), 6.61(\mathrm{~d}, J$ $=8.2 \mathrm{~Hz}, 2 \mathrm{H}), 3.70(\mathrm{~s}, 3 \mathrm{H}), 2.59(\mathrm{~s}, 12 \mathrm{H}), 2.29(\mathrm{q}, J=12.0 \mathrm{~Hz}, 2 \mathrm{H}), 2.02-1.95(\mathrm{~m}, 2 \mathrm{H}), 1.80-$ $1.68(\mathrm{~m}, 6 \mathrm{H}), 1.65(\mathrm{~d}, J=13.6 \mathrm{~Hz}, 2 \mathrm{H}), 1.55-1.46(\mathrm{~m}, 2 \mathrm{H}), 1.34-1.05(\mathrm{~m}, 7 \mathrm{H}), 0.96-0.84$ $(\mathrm{m}, 3 \mathrm{H})$.

${ }^{13} \mathbf{C}$ NMR $\left(126 \mathrm{MHz}, \mathrm{CD}_{2} \mathrm{Cl}_{2}\right) \delta 157.3,156.2,147.2,147.0,136.5,135.2,134.9,134.5,134.3$, 134.2, 133.6, 130.8, 126.4, 126.2, 118.8, 116.2, 113.5, 55.5, 45.3, 37.0, 36.8, 29.5, 29.4, 28.1, 28.0, 27.9, 27.8, 26.3.

${ }^{31}$ P NMR $\left(203 \mathrm{MHz}, \mathrm{CD}_{2} \mathrm{Cl}_{2}\right) \delta 31.93$.

M.P. $176-179^{\circ} \mathrm{C}(\mathrm{dec}$.)

IR (neat): 2932, 2845, 2821, 2778, 1566, 1476, 1459, 1447, 1294, 1268, 1234, 1175, 1109, 1099 , $1061,1040,1026,1005,931,893,848,806,794,744,590 \mathrm{~cm}^{-1}$

EA Calcd. for $\mathrm{C}_{35} \mathrm{H}_{48} \mathrm{ClN}_{2} \mathrm{OPPd}$ : C, 61.31; H, 7.06. Found: C, 61.59; H, 7.28. 
$(t$-BuXPhos)Pd(X4)Br (11)

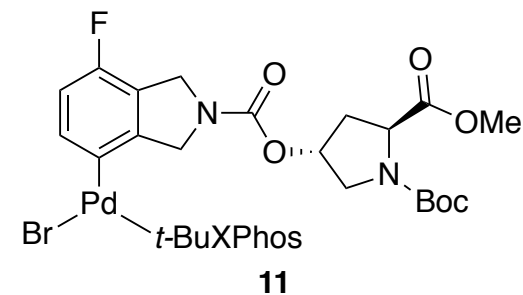

Compound 11 was prepared according to a modified General Procedure A using $\mathbf{P 3}$ (208 mg, $0.600 \mathrm{mmol}, 1.20$ equiv), $t$ BuXPhos (306 mg, $0.725 \mathrm{mmol}, 1.44$ equiv), X4 (244 mg, $0.500 \mathrm{mmol}, 1.00$ equiv), and THF (5.0 mL, anhydrous). After $2 \mathrm{~h}$, the reaction was allowed to cool to room temperature, opened to air, and filtered through a $\sim 1 \mathrm{~cm}$ plug of Celite ${ }^{\circledR}$ into a $100 \mathrm{~mL}$ round bottom flask. The Celite ${ }^{\circledR}$ plug was

further washed with THF ( $25 \mathrm{~mL}$, stabilized). The filtrate was collected and concentrated in vacuo with the assistance of a rotary evaporator to yield a teal-colored viscous oil. The oil was layered with pentane $(50 \mathrm{~mL})$ and triturated with the assistance of sonication for $\sim 5 \mathrm{~min}$ to form a teal precipitate. The precipitate was collected on a fritted funnel and washed with pentane $(50 \mathrm{~mL})$ to yield 11 (323 mg, 63\%) as a teal powder. The ${ }^{1} \mathrm{H}$ NMR, ${ }^{13} \mathrm{C}$ NMR, ${ }^{19} \mathrm{~F}$ NMR, ${ }^{31} \mathrm{P}$ NMR and IR spectroscopic data and melting point matched that reported in the literature. ${ }^{17}$ This complex is a mixture of diastereomers and rotamers.

${ }^{1} \mathbf{H}$ NMR $\left(600 \mathrm{MHz}, \mathrm{CD}_{2} \mathrm{Cl}_{2}\right) \delta 8.02(\mathrm{tt}, J=6.0,2.8 \mathrm{~Hz}, 1 \mathrm{H}), 7.44-7.37(\mathrm{~m}, 2 \mathrm{H}), 7.11-7.04$ $(\mathrm{m}, 2 \mathrm{H}), 7.01-6.95(\mathrm{~m}, 1 \mathrm{H}), 6.74-6.69(\mathrm{~m}, 1 \mathrm{H}), 6.63(\mathrm{td}, J=8.9,4.1 \mathrm{~Hz}, 1 \mathrm{H}), 5.28-5.17(\mathrm{~m}$, $1 \mathrm{H}), 4.80-4.66(\mathrm{~m}, 2 \mathrm{H}), 4.61(\mathrm{~s}, 2 \mathrm{H}), 4.42-4.30(\mathrm{~m}, 1 \mathrm{H}), 3.76-3.51(\mathrm{~m}, 5 \mathrm{H}), 3.12-3.04(\mathrm{~m}$, $1 \mathrm{H}), 2.60-2.50(\mathrm{~m}, 2 \mathrm{H}), 2.49-2.40(\mathrm{~m}, 1 \mathrm{H}), 2.27-2.15(\mathrm{~m}, 1 \mathrm{H}), 1.63(\mathrm{dd}, J=6.9,3.4 \mathrm{~Hz}$, $3 \mathrm{H}), 1.53(\mathrm{dd}, J=14.1,4.7 \mathrm{~Hz}, 8 \mathrm{H}), 1.50-1.46(\mathrm{~m}, 4 \mathrm{H}), 1.45-1.42(\mathrm{~m}, 3 \mathrm{H}), 1.42-1.35(\mathrm{~m}$, $12 \mathrm{H}), 1.32(\mathrm{dd}, J=14.1,5.8 \mathrm{~Hz}, 9 \mathrm{H}), 0.94-0.89(\mathrm{~m}, 3 \mathrm{H}), 0.83(\mathrm{t}, J=6.7 \mathrm{~Hz}, 3 \mathrm{H})$.

${ }^{13} \mathrm{C}$ NMR $\left(151 \mathrm{MHz}, \mathrm{CD}_{2} \mathrm{Cl}_{2}\right) \delta 173.6,173.6,173.3,173.3,173.2,158.9,158.8,158.8,157.6$, 157.5, 156.0, 155.9, 154.6, 154.4, 154.4, 154.3, 154.2, 154.2, 153.9, 153.9, 153.9, 153.8, 153.7, 153.7, 153.6, 153.6, 153.6, 147.3, 147.3, 147.2, 147.1, 146.4, 146.1, 137.6, 136.4, 136.4, 136.2, $136.2,135.7,135.3,135.2,130.6,130.6,126.2,126.2,126.2,126.1,125.5,125.5,125.4,125.1$, $125.0,125.0,125.0,124.6,124.6,124.5,124.5,124.5,121.8,121.7,121.6,121.6,121.5,121.4$, $120.8,120.7,120.2,118.1,117.9,112.1,112.0,111.8,80.5,80.5,80.4,80.4,80.4,80.30,80.3$, 73.8, 73.8, 73.7, 73.2, 73.1, 73.1, 73.1, 58.7, 58.4, 58.4, 58.3, 58.2, 58.1, 58.1, 58.0, 53.2, 52.8, 52.7, 52.6, 52.4, 52.4, 50.4, 50.4, 50.0, 50.0, 39.7, 39.7, 39.6, 39.6, 39.3, 39.2, 37.4, 37.3, 37.3, $36.4,36.3,36.3,36.1,34.8,32.3,32.3,32.1,32.1,32.1,31.6,30.9,30.9,30.9,30.9,30.8,30.8$, $28.5,28.5,28.4,28.4,28.3,28.1,27.1,26.5,25.8,25.8,25.7,25.7,25.7,25.0,25.0,24.9,24.9$, $24.5,24.2,23.8,23.7,23.7,23.7,23.6$.

${ }^{19}$ F NMR $\left(565 \mathrm{MHz}, \mathrm{CD}_{2} \mathrm{Cl}_{2}\right) \delta-129.98,-130.03,-130.19,-130.27$.

${ }^{31} \mathbf{P}$ NMR $\left(203 \mathrm{MHz}, \mathrm{CD}_{2} \mathrm{Cl}_{2}\right) \delta 53.77,53.63-52.98(\mathrm{~m})$.

M.P. $150-155^{\circ} \mathrm{C}$ (dec.). Lit.: $150-155^{\circ} \mathrm{C}$ (dec.)

IR (neat): 2965, 2930, 2868, 1751, 1706, 1604, 1462, 1414, 1391, 1362, 1331, 1315, 1244, 1199, $1175,1159,1099,1071,1019,995,938,924,898,870,803,768,758,732,694,680 \mathrm{~cm}^{-1}$

\section{(t-BuXPhos)Pd(Rivaroxaban)Cl (12)}

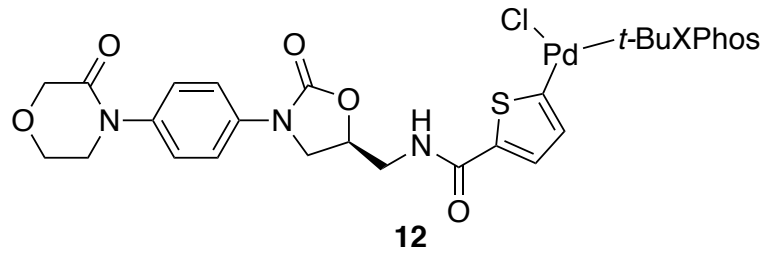

Compound 12 was prepared according to the General Procedure B using P3 (208 mg, 0.600 mmol, 1.20 equiv), $t$-BuXPhos (297 mg, 0.700 mmol, 1.40 equiv), Rivaroxaban (218 mg, $0.500 \mathrm{mmol}, 1.00$ equiv), 2-MeTHF (2.5 mL), and THF (5.0 $\mathrm{mL}$, anhydrous). The precipitate 
was collected on a fritted funnel to yield $12(388 \mathrm{mg}, 80 \%)$ as a pale-green powder. The ${ }^{1} \mathrm{H} \mathrm{NMR}$, ${ }^{13} \mathrm{C} \mathrm{NMR},{ }^{31} \mathrm{P} \mathrm{NMR}$ and IR spectroscopic data and melting point matched that reported in the literature. ${ }^{17}$

${ }^{1} \mathbf{H}$ NMR $\left(600 \mathrm{MHz}, \mathrm{CD}_{2} \mathrm{Cl}_{2}\right) \delta 7.95(\mathrm{td}, J=6.6,2.3 \mathrm{~Hz}, 1 \mathrm{H}), 7.58(\mathrm{~d}, J=8.6 \mathrm{~Hz}, 2 \mathrm{H}), 7.47-$ $7.39(\mathrm{~m}, 2 \mathrm{H}), 7.20-7.11(\mathrm{~m}, 5 \mathrm{H}), 6.76(\mathrm{dt}, J=6.4,2.9 \mathrm{~Hz}, 1 \mathrm{H}), 6.59(\mathrm{~d}, J=3.8 \mathrm{~Hz}, 1 \mathrm{H}), 4.81-$ $4.74(\mathrm{~m}, 1 \mathrm{H}), 4.26-4.16(\mathrm{~m}, 3 \mathrm{H}), 4.00-3.87(\mathrm{~m}, 3 \mathrm{H}), 3.78(\mathrm{dd}, J=9.5,5.9 \mathrm{~Hz}, 1 \mathrm{H}), 3.58$ (br s, 2H), 3.31 (br s, 1H), 3.04 (hept, $J=6.9 \mathrm{~Hz}, 1 \mathrm{H}), 2.67$ (br s, 1H), 2.42 (br s, 1H), 1.67 - 1.62 (m, $4 \mathrm{H}), 1.57-1.45(\mathrm{~m}, 12 \mathrm{H}), 1.41-1.29(\mathrm{~m}, 15 \mathrm{H}), 0.90(\mathrm{t}, J=6.3 \mathrm{~Hz}, 6 \mathrm{H})$.

${ }^{13} \mathrm{C}$ NMR $\left(151 \mathrm{MHz}, \mathrm{CD}_{2} \mathrm{Cl}_{2}\right) \delta 166.8,162.3,159.7,154.9,154.5,147.4,147.3,141.1,137.4$, $136.6,136.5,135.3,135.2,134.8,134.7,131.0,131.0,129.0,126.5,126.5,126.2,125.7,125.3$, $122.2,122.2,119.2,118.9,71.9,68.9,64.5,49.9,41.9,40.2,40.1,35.2,32.2,31.8,31.3,31.3$, $30.7,25.6,25.6,24.9,24.8,24.8,24.6$.

${ }^{31} \mathbf{P}$ NMR $\left(203 \mathrm{MHz}, \mathrm{CD}_{2} \mathrm{Cl}_{2}\right) \delta 58.74$.

M.P. $165-168^{\circ} \mathrm{C}$ (dec.). Lit.: $165-168^{\circ} \mathrm{C}$ (dec.)

IR (neat): 2965, 2923, 2868, 1753, 1665, 1642, 1601, 1511, 1473, 1464, 1421, 1393, 1341, 1310, $1284,1220,1163,1125,1069,1054,1024,993,938,919,874,827,803,773,747,732,709,683$, $640,619,600 \mathrm{~cm}^{-1}$

\section{(t-BuXPhos)Pd(Gefitinib)Cl (13)}

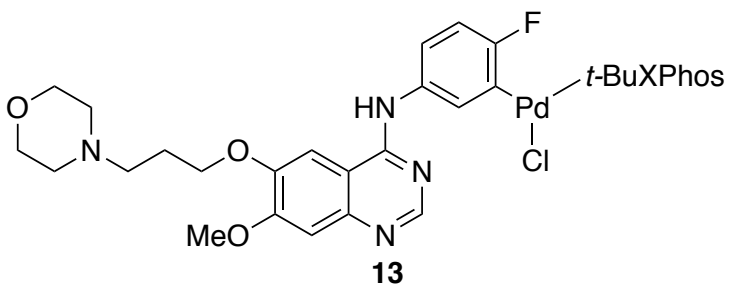

Compound 13 was prepared according to the General Procedure B using P3 (208 mg, 0.600 mmol, 1.20 equiv), $t$-BuXPhos (297 $\mathrm{mg}, 0.700$ mmol, 1.40 equiv), Gefitinib (224 mg, 0.500 mmol, 1.00 equiv), 2-MeTHF (2.5 mL), and THF (5.0 $\mathrm{mL}$, anhydrous). The precipitate was collected on a fritted funnel to yield $13(429 \mathrm{mg}$, $88 \%$ ) as a pale-red powder. The ${ }^{1} \mathrm{H}$ NMR, ${ }^{13} \mathrm{C}$ NMR, ${ }^{19} \mathrm{~F}$ NMR, ${ }^{31} \mathrm{P}$ NMR and IR spectroscopic data and melting point matched that reported in the literature. ${ }^{17}$

${ }^{1} \mathbf{H}$ NMR $\left(500 \mathrm{MHz}, \mathrm{CD}_{2} \mathrm{Cl}_{2}\right.$ ) Complex spectrum, see below.

${ }^{13} \mathrm{C}$ NMR $\left(151 \mathrm{MHz}, \mathrm{CD}_{2} \mathrm{Cl}_{2}\right) \delta 160.9,159.1,156.8,155.2,154.3,153.8,153.8,149.2,147.7$, $147.6,137.0,136.8,135.6,134.9,134.8,133.6,133.2,130.7,126.2$, 126.2, 125.2, 125.1, 124.7, 123.0, 119.4, 113.5, 113.3, 109.3, 108.2, 101.4, 68.0, 67.4, 67.4, 67.3, 56.5, 55.5, 54.2, 39.7, $39.6,39.6,39.5,35.1,32.5,32.1,32.1,32.0,32.0,31.9,31.1,31.1,26.7,25.8,25.8,24.9,24.8$, 24.7, 23.6, 23.5.

${ }^{19}$ F NMR $\left(471 \mathrm{MHz}, \mathrm{CD}_{2} \mathrm{Cl}_{2}\right) \delta-91.84$.

${ }^{31} \mathbf{P}$ NMR $\left(203 \mathrm{MHz}, \mathrm{CD}_{2} \mathrm{Cl}_{2}\right) \delta 55.69,55.66$.

M.P. $190-192{ }^{\circ} \mathrm{C}($ dec. $)$; turns black $175^{\circ} \mathrm{C}$. Lit.: $170-175^{\circ} \mathrm{C}($ dec. $)$

IR (neat): 2958, 2930, 2866, 1620, 1604, 1566, 1500, 1466, 1424, 1383, 1360, 1334, 1249, 1206, $1166,1137,1118,1066,1033,1009,936,917,855,810,768,754,747,735,661 \mathrm{~cm}^{-1}$ 
(XPhos)Pd(Rivaroxaban)Cl (14)

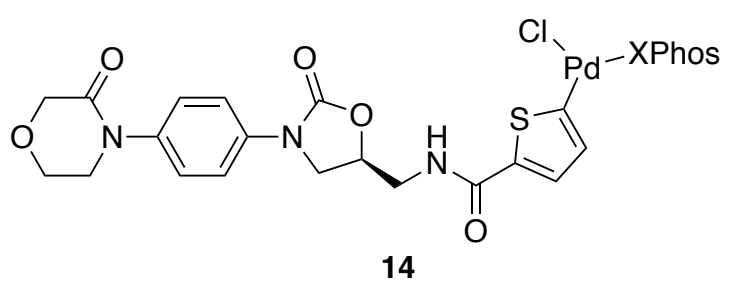

Compound $\mathbf{1 4}$ was prepared according to a modified General Procedure B using P3 (208 mg, $0.600 \mathrm{mmol}, 1.20$ equiv), XPhos ( $387 \mathrm{mg}, 0.720$ mmol, 1.44 equiv), Rivaroxaban ( $218 \mathrm{mg}, 0.500$ mmol, 1.00 equiv), 2-MeTHF ( $2.5 \mathrm{~mL})$, and THF (5.0 $\mathrm{mL}$, anhydrous). Following the addition of THF, the reaction mixture was allowed to stir for

$24 \mathrm{~h}$ at room temperature. The precipitate was collected on a fritted funnel to yield $\mathbf{1 4}$ (368 $\mathrm{mg}$, $72 \%)$ as a pale-tan powder.

${ }^{1}$ H NMR $\left(500 \mathrm{MHz}, \mathrm{CD}_{2} \mathrm{Cl}_{2}\right.$ ) Complex spectrum, see below.

${ }^{13}$ C NMR $\left(151 \mathrm{MHz}, \mathrm{CD}_{2} \mathrm{Cl}_{2}\right) \delta 167.1,167.0,166.9,166.9,162.6,162.5,161.9,158.5,155.0$, 155.0, 154.8, 154.7, 154.7, 151.9, 151.8, 149.5, 149.4, 149.4, 146.6, 146.5, 142.4, 141.5, 141.4, $140.9,140.0,137.7,137.7,137.6,137.6,137.4,137.3,137.3,137.2,135.7,134.8,134.6,134.4$, $134.4,133.8,133.7,132.7,132.5,132.1,131.8,131.5,130.9,130.1,129.5,129.3,128.8,128.6$, $128.3,128.2,127.7,127.5,127.5,126.6,126.6,126.4,126.4,126.0,125.9,125.3,125.2,121.6$, $120.4,119.2,119.2,119.1,119.0,72.6,72.4,72.3,72.1,68.9,68.1,64.5,64.5,50.0,50.0,50.0$, $48.5,48.2,48.2,42.8,42.2,36.1,35.9,35.0,34.8,34.5,33.8,32.1,32.1,31.2,30.8,29.0,28.4$, $28.3,28.1,27.9,27.8,27.8,27.5,27.4,27.2,27.1,27.1,27.0,26.9,26.7,26.4,26.3,26.2,26.1$, 26.0, 26.0, 25.8, 25.8, 25.6, 25.5, 25.4, 24.9, 24.8, 24.4, 24.4, 24.3, 24.2, 24.2.

${ }^{31}$ P NMR $\left(203 \mathrm{MHz}, \mathrm{CD}_{2} \mathrm{Cl}_{2}\right) \delta 36.28$.

M.P. $192-196$ (dec.)

IR (neat): 2958, 2925, 2849, 1758, 1661, 1649, 1606, 1514, 1464, 1445, 1400, 1381, 1360, 1341, $1308,1282,1237,1215,1128,1066,1050,998,936,922,879,848,832,806,784,765,739,706$ $\mathrm{cm}^{-1}$

EA Calcd. for $\mathrm{C}_{52} \mathrm{H}_{67} \mathrm{ClN}_{3} \mathrm{O}_{5} \mathrm{PPdS}$ : C, 61.29; H, 6.63. Found: C, 61.02; H, 6.53.

\section{(BrettPhos)Pd(Rivaroxaban)Cl (15)}
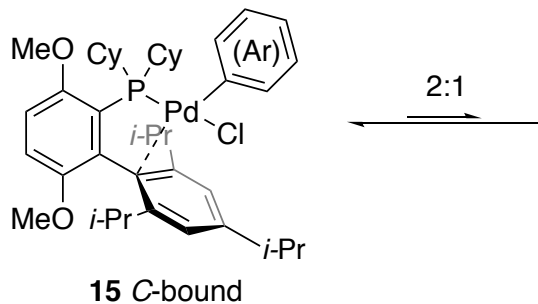
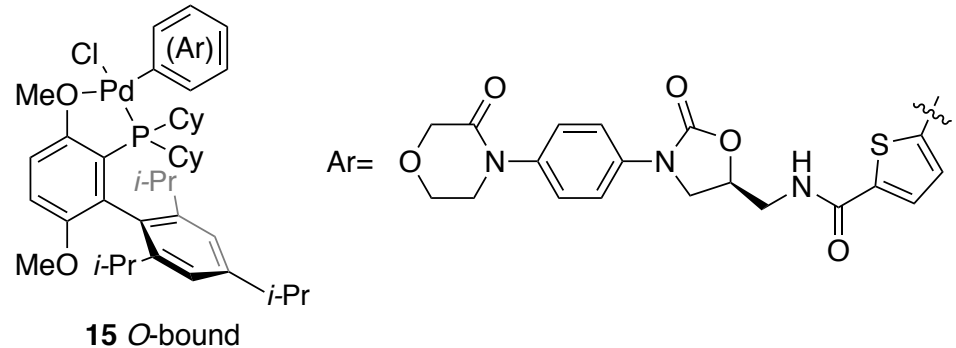

$15 O$-bound

Compound 15 was prepared according to a modified General Procedure B using P3 (208 mg, 0.600 mmol, 1.20 equiv), BrettPhos ( $387 \mathrm{mg}, 0.720 \mathrm{mmol}, 1.44$ equiv), Rivaroxaban ( $218 \mathrm{mg}, 0.500$ mmol, 1.00 equiv), 2-MeTHF ( $2.5 \mathrm{~mL})$, and THF ( $5.0 \mathrm{~mL}$, anhydrous). Following the addition of THF, the reaction mixture was allowed to stir for $2 \mathrm{~h}$ at room temperature. The precipitate was collected on a fritted funnel to yield $\mathbf{1 5}(529 \mathrm{mg}, 98 \%)$ as a pale-lavender powder. Compound $\mathbf{1 5}$ was determined to be a 2:1 mixture of $C$-Bound to $O$-Bound isomer.

${ }^{1} \mathrm{H}$ NMR $\left(500 \mathrm{MHz}, \mathrm{CD}_{2} \mathrm{Cl}_{2}\right.$ ) Complex spectrum, see below. 
${ }^{13} \mathrm{C}$ NMR $\left(126 \mathrm{MHz}, \mathrm{CD}_{2} \mathrm{Cl}_{2}\right) \delta 166.9,166.8,162.7,162.5,159.3,157.1,157.0,155.0,155.0$, $154.9,154.8,154.8,154.8,154.5,154.3,152.3,152.2,149.8,147.3,147.3,146.6,140.4,140.4$, $139.8,139.8,138.8,138.2$, 138.1, 137.6, 137.5, 137.4, 133.8, 133.7, 133.5, 133.5, 132.8, 132.7, $130.5,129.2,128.6,126.4,126.3,125.2,125.0,124.9,124.8,124.1,122.1,122.0,119.9,119.3$, $119.1,118.9,117.5,117.3,114.8,114.7,114.6,114.4,114.4,112.0,112.0,111.9,111.8,72.3$, $72.1,68.9,64.5,62.5,55.4,55.3,54.8,50.0,50.0,48.8,48.5,42.3,42.0,40.9,40.3,36.7,36.6$, $36.5,36.5,36.4,36.3,36.1,35.9,35.1,34.7,34.1,32.3,32.1,32.0,31.4,31.3,31.3,31.3,31.1$, $30.5,30.2,30.0,29.3,28.9,28.4,28.2,28.1,28.1,28.0,28.0,27.9,27.8,27.7,27.7,27.6,27.6$, $27.5,27.4,27.4,27.3,27.3,27.3,27.2,27.0,26.9,26.8,26.7,26.7,26.6,26.6,26.4,26.2,26.2$, $26.0,25.9,25.7,25.6,25.6,25.2,25.2,25.0,24.8,24.6,24.6,24.4,24.4,24.2,24.1,24.1,23.9$, 23.6, 23.5, 23.4 .

${ }^{31} \mathbf{P}$ NMR $\left(203 \mathrm{MHz}, \mathrm{CD}_{2} \mathrm{Cl}_{2}\right) \delta 57.91,46.13$.

M.P. $187-190{ }^{\circ} \mathrm{C}($ dec. $)$

IR (neat): 3323, 2956, 2925, 2854, 1755, 1663, 1646, 1606, 1580, 1514, 1459, 1421, 1393, 1381, $1362,1341,1308,1253,1217,1121,1080,1052,1012,998,941,919,889,877,851,832,803$, $751,709,690,649 \mathrm{~cm}^{-1}$

EA: Calcd. for $\mathrm{C}_{54} \mathrm{H}_{71} \mathrm{ClN}_{3} \mathrm{O}_{7} \mathrm{PPdS}$ : C, 60.11; H, 6.63. Found: C, 60.22; H, 6.89.

\section{(RuPhos)Pd(2-Et-6-Me-3-pyridyl)OTf (16)}

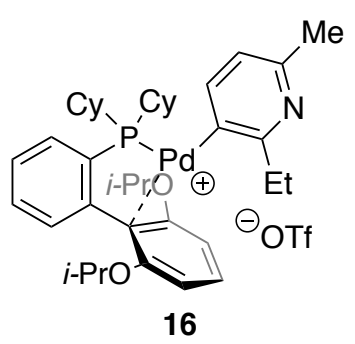

Compound 16 was prepared according to a modified General Procedure A using $\mathbf{P 3}$ (173 mg, $0.500 \mathrm{mmol}, 1$ equiv), RuPhos (223 mg, 0.525 mmol, $\quad 1.05 \quad$ equiv), 2-ethyl-6-methylpyridin-3-yl trifluoromethanesulfonate (202 $\mathrm{mg}, 0.750 \mathrm{mmol}, 1.50$ equiv), and $n$ hexane $(5.0 \mathrm{~mL})$. The precipitate was collected on a fritted funnel to yield 16 (379 $\mathrm{mg}, 90 \%$ ) as a pale-pink powder. The ${ }^{1} \mathrm{H} \mathrm{NMR},{ }^{13} \mathrm{C} \mathrm{NMR}$, ${ }^{19} \mathrm{~F}$ NMR, ${ }^{31} \mathrm{P}$ NMR and IR spectroscopic data matched that reported in the literature. ${ }^{18}$

${ }^{1} \mathbf{H}$ NMR $\left(500 \mathrm{MHz}, \mathrm{CD}_{3} \mathrm{CN}\right) \delta 7.75-7.68(\mathrm{~m}, 2 \mathrm{H}), 7.53(\mathrm{tt}, J=7.5,1.6 \mathrm{~Hz}, 1 \mathrm{H}), 7.47(\mathrm{tt}, J=$ 7.4, $1.7 \mathrm{~Hz}, 1 \mathrm{H}), 6.99(\mathrm{dd}, J=7.9,2.8 \mathrm{~Hz}, 1 \mathrm{H}), 6.85-6.75(\mathrm{~m}, 4 \mathrm{H}), 4.72$ (hept, $J=6.1 \mathrm{~Hz}, 1 \mathrm{H})$, 4.64 (hept, $J=6.0 \mathrm{~Hz}, 1 \mathrm{H}), 3.18$ (hept, $J=7.1 \mathrm{~Hz}, 2 \mathrm{H}), 2.46-2.38(\mathrm{~m}, 2 \mathrm{H}), 2.36$ (s, 3H), $2.16-$ $2.07(\mathrm{~m}, 1 \mathrm{H}), 1.94(\mathrm{dt}, J=4.9,2.5 \mathrm{~Hz}, 2 \mathrm{H}), 1.91-1.77(\mathrm{~m}, 2 \mathrm{H}), 1.76-1.68(\mathrm{~m}, 1 \mathrm{H}), 1.67-$

$1.59(\mathrm{~m}, 1 \mathrm{H}), 1.57-1.43(\mathrm{~m}, 5 \mathrm{H}), 1.41(\mathrm{~d}, J=6.0 \mathrm{~Hz}, 3 \mathrm{H}), 1.35(\mathrm{~d}, J=6.0 \mathrm{~Hz}, 3 \mathrm{H}), 1.30-1.22$ $(\mathrm{m}, 4 \mathrm{H}), 1.22-1.12(\mathrm{~m}, 5 \mathrm{H}), 1.09(\mathrm{~d}, J=6.1 \mathrm{~Hz}, 5 \mathrm{H}), 0.93(\mathrm{~d}, J=6.0 \mathrm{~Hz}, 3 \mathrm{H}), 0.18(\mathrm{qt}, J=$ $12.7,4.2 \mathrm{~Hz}, 1 \mathrm{H})$.

${ }^{13}$ C NMR $\left(126 \mathrm{MHz}, \mathrm{CD}_{3} \mathrm{CN}\right) \delta 164.7,164.4,164.1,154.7,145.6,145.5,143.6,143.5,139.0$, $135.5,135.2,133.1,133.0,132.5,132.4,132.3,130.7,128.1,128.1,122.8,106.9,106.7,73.2$, 72.2, 36.8, 35.7, 35.4, 33.6, 33.4, 31.9, 31.9, 29.4, 29.4, 27.8, 27.7, 27.6, 27.6, 27.4, 27.1, 27.0, 26.9, 26.8, 26.7, 26.6, 26.6, 23.4, 22.4, 22.2, 22.1, 21.8, 13.3.

${ }^{19}$ F NMR $\left(471 \mathrm{MHz}, \mathrm{CD}_{3} \mathrm{CN}\right) \delta-78.65$.

${ }^{31} \mathbf{P}$ NMR $\left(203 \mathrm{MHz}, \mathrm{CD}_{3} \mathrm{CN}\right) \delta 41.30$.

M.P. $194-196^{\circ} \mathrm{C}($ dec. $)$

IR (neat): 2979, 2930, 2920, 2861, 1587, 1568, 1450, 1445, 1421, 1383, 1374, 1310, 1253, 1227 , $1204,1168,1154,1106,1066,1012,995,893,846,818,803,775,761,749,735,628 \mathrm{~cm}^{-1}$ 


\section{4,4'-[(RuPhos)Pd(Br)]2(biphenyl) (17)}

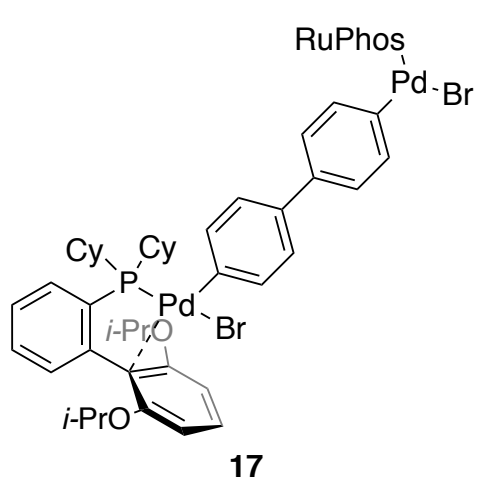

Compound 17 was prepared according to a modified General Procedure A using P3 (208 mg, $0.600 \mathrm{mmol}, 2.40$ equiv), RuPhos (308 mg, 0.660 mmol, 2.64 equiv), 4,4'dibromobiphenyl (78 mg, $0.250 \mathrm{mmol}, 1.00$ equiv), and cyclohexane $(5.0 \mathrm{~mL})$. The reaction mixture was allowed to stir at $60{ }^{\circ} \mathrm{C}$ for $24 \mathrm{~h}$, during which time the mixture became homogeneous. Subsequently a pale-tan powder precipitated (mono-addition) that eventually turned into a medium-grey powder (desired double-addition). The reaction mixture was then removed from the oil bath and allowed to cool to room temperature. The mixture was opened to air and diluted with pentane $(10 \mathrm{~mL})$ to assist with precipitation. The precipitate was collected on a fritted funnel and washed with pentane $(25 \mathrm{~mL})$ to yield $\mathbf{1 7}(340 \mathrm{mg}, 93 \%)$ as a grey powder. The ${ }^{1} \mathrm{H} \mathrm{NMR},{ }^{13} \mathrm{C}$ NMR, ${ }^{31} \mathrm{P}$ NMR and IR spectroscopic data matched that reported in the literature. ${ }^{19}$

${ }^{1} \mathbf{H}$ NMR $\left(600 \mathrm{MHz}, \mathrm{CD}_{2} \mathrm{Cl}_{2}\right) \delta 7.64(\mathrm{t}, J=8.4 \mathrm{~Hz}, 4 \mathrm{H}), 7.44(\mathrm{tt}, J=7.5,1.5 \mathrm{~Hz}, 2 \mathrm{H}), 7.39$ (tt, $J$ $=7.3,1.4 \mathrm{~Hz}, 2 \mathrm{H}), 7.20-7.15(\mathrm{~m}, 4 \mathrm{H}), 7.10(\mathrm{dd}, J=8.4,2.2 \mathrm{~Hz}, 4 \mathrm{H}), 6.87$ (ddd, $J=7.8,3.1$, $1.4 \mathrm{~Hz}, 2 \mathrm{H}), 6.66(\mathrm{~d}, J=8.4 \mathrm{~Hz}, 4 \mathrm{H}), 4.63$ (hept, $J=6.0 \mathrm{~Hz}, 4 \mathrm{H}), 2.23-2.14$ (m, 4H), $1.83-$ $1.73(\mathrm{~m}, 12 \mathrm{H}), 1.70-1.55(\mathrm{~m}, 13 \mathrm{H}), 1.45-1.39(\mathrm{~m}, 14 \mathrm{H}), 1.29-1.17(\mathrm{~m}, 9 \mathrm{H}), 1.17-1.07(\mathrm{~m}$, $4 \mathrm{H}), 1.03(\mathrm{~d}, J=6.1 \mathrm{~Hz}, 12 \mathrm{H}), 0.79$ (qt, $J=13.1,3.8 \mathrm{~Hz}, 4 \mathrm{H})$.

${ }^{13}$ C NMR $\left(151 \mathrm{MHz}, \mathrm{CD}_{2} \mathrm{Cl}_{2}\right) \delta 159.4,145.2,145.1,138.0,137.9,136.6,134.8,134.8,134.0$, $133.8,132.8,132.8,132.0,131.4,130.9,130.8,128.7,126.6,126.6,125.2,111.8,111.8,107.7$, $107.6,71.3,71.2,34.1,34.0,28.5,28.0,27.9,27.6,27.5,27.3,27.2,27.1,26.4,22.2,21.8$.

${ }^{31} \mathbf{P}$ NMR $\left(203 \mathrm{MHz}, \mathrm{CD}_{2} \mathrm{Cl}_{2}\right) \delta 31.80$.

M.P. $230-233{ }^{\circ} \mathrm{C}(\mathrm{dec}$.

IR (neat): $3053,3008,2979,2918,2849,1585,1575,1450,1440,1383,1369,1341,1331,1282$, 1253, 1206, 1194, 1182, 1173, 1135, 1106, 1061, 993, 943, 929, 915, 893, 848, 815, 808, 796, $784,775,768,758,747,737,728,711,702,675,654,628,609,602 \mathrm{~cm}^{-1}$

\section{(BrettPhos)Pd(4-I-Ph)I (18)}

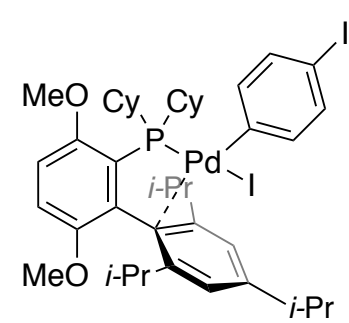

$18 C$-bound

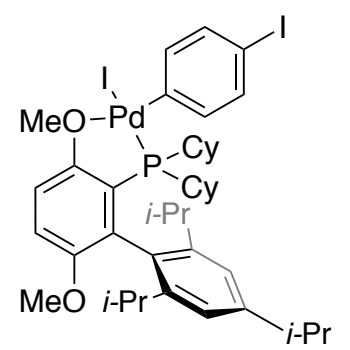

18 O-bound

Compound 18 was prepared according to a modified General Procedure B. An oven-dried $24 \mathrm{~mL}$ reaction tube, $\mathbf{A}$, was equipped, while still hot, with a magnetic stir bar and septum cap and was allowed to cool to room temperature under vacuum by piercing the septum with a needle connected to a dual manifold by a rubber hose. The cap was removed and the tube was charged with P3 (173 $\mathrm{mg}, 0.500 \mathrm{mmol}, 1.00$ equiv) and BrettPhos (322 $\mathrm{mg}, 0.600 \mathrm{mmol}, 1.20$ equiv). The tube was 
recapped, pierced with a needle and reconnected to the dual manifold, evacuated and backfilled with argon (the evacuation/backfill process was repeated a total of three times). Under argon, the tube was charged with 2-MeTHF $(2.5 \mathrm{~mL})$ via syringe. The tube was then placed in a pre-heated oil bath (bath temperature $=60^{\circ} \mathrm{C}$ ). The reaction mixture was allowed to stir at $60{ }^{\circ} \mathrm{C}$ for $30 \mathrm{~min}$. Over this period, the mixture became homogeneous and subsequently [(BrettPhos)Pd $]_{2}(\mathrm{cod})$ precipitated. The reaction tube was then removed from the oil bath and the mixture was allowed to cool to room temperature under argon for $10 \mathrm{~min}$. A second oven-dried $8 \mathrm{~mL}$ reaction tube, $\mathbf{B}$, was equipped, while still hot, with a septum cap and allowed to cool to room temperature under vacuum. The cap was then removed and the tube was charged with diiodobenzene (495 $\mathrm{mg}, 1.50$ mmol, 3.00 equiv). The tube was recapped, pierced with a needle connected to the dual manifold, evacuated and backfilled with argon (the evacuation/backfill process was repeated a total of three times). Under argon, 2-MeTHF (2.5 mL) was added to $\mathbf{B}$ via syringe. The contents of tube $\mathbf{B}$ were transferred to tube $\mathbf{A}$ via syringe. Upon complete addition of the aryl halide solution to $\mathbf{A}$, the mixture was allowed to stir at room temperature under argon for $1 \mathrm{~h}$, during which time a yellow precipitate formed. The reaction mixture was opened to air and diluted with pentane $(10 \mathrm{~mL})$ to assist with precipitation. The precipitate was collected on a fritted funnel and washed with pentane $(15 \mathrm{~mL})$ and dried in vacuo on the Schlenk line overnight $(\sim 16 \mathrm{~h})$ to yield $\mathbf{1 8}(387 \mathrm{mg}, 80 \%)$ as a shimmery pale-yellow powder. Compound $\mathbf{1 8}$ was determined to be a 5:1 mixture of $C$-Bound to $O$-Bound isomer. The ${ }^{1} \mathrm{H}$ NMR, ${ }^{13} \mathrm{C} \mathrm{NMR},{ }^{31} \mathrm{P} \mathrm{NMR}$ and IR spectroscopic data matched that reported in the literature. ${ }^{20}$

${ }^{1} \mathbf{H}$ NMR (600 MHz, $\left.\mathrm{CDCl}_{3}\right)$ Complex spectrum, see below.

${ }^{13} \mathrm{C}$ NMR $\left(151 \mathrm{MHz}, \mathrm{CDCl}_{3}\right) \delta 157.3,156.7,156.6,154.8,154.8,154.0,153.9,152.3,152.0$, $151.9,149.2,146.8,141.1,141.1,140.9,140.9,138.1,138.0,134.8,134.7,133.5,133.5,131.6$, 131.6, 130.3, 125.3, 124.7, 124.6, 121.5, 118.9, 118.7, 116.9, 116.9, 113.3, 113.3, 113.1, 111.6, $111.5,111.1,111.1,88.2,88.0,64.9,55.1,55.1,54.8,54.5,36.7,36.5,35.3,35.1,34.4,34.4$, $34.3,34.2,31.6,31.0,30.7,30.7,29.4,29.4,29.1,29.1,28.0,28.0,27.9,27.9,26.9,26.8,26.6$, $26.5,26.2,25.6,25.5,25.4,24.9,24.6,24.1,23.5,22.5,14.2$.

${ }^{31} \mathbf{P}$ NMR $\left(203 \mathrm{MHz}, \mathrm{CDCl}_{3}\right) \delta 41.67,31.82$.

M.P. $187-190{ }^{\circ} \mathrm{C}$ (dec.)

IR (neat): 2951, 2923, 2852, 1604, 1573, 1537, 1459, 1447, 1419, 1383, 1360, 1239, 1201, 1185, $1161,1061,1040,990,874,851,820,796,765,749,713 \mathrm{~cm}^{-1}$

\section{(SPhos)Pd[4-CH2 $\left.\mathrm{CH}_{2} \mathrm{C}(\mathrm{O})(\mathrm{NHS}) \mathrm{Ph}\right] \mathrm{Br}$ (19)}

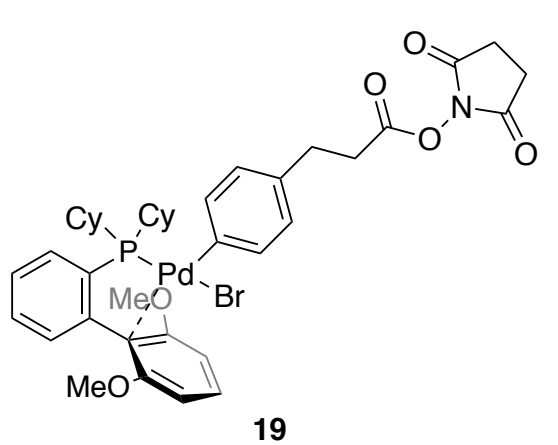

Compound 19 was prepared according to a modified General Procedure A using P3 (182 mg, $0.525 \mathrm{mmol}, 1.05$ equiv), SPhos (226 mg, $0.550 \mathrm{mmol}, 1.10$ equiv), 2,5dioxopyrrolidin-1-yl 3-(4-bromophenyl)propanoate (163 $\mathrm{mg}, 0.500 \mathrm{mmol}, 1.00$ equiv), and cyclohexane $(5.0 \mathrm{~mL})$. The reaction mixture was allowed to stir at $60^{\circ} \mathrm{C}$ for $16 \mathrm{~h}$. The precipitate was collected on a fritted funnel to yield 19 (408 $\mathrm{mg}, 97 \%$ ) as a pale-tan powder. The ${ }^{1} \mathrm{H} \mathrm{NMR},{ }^{13} \mathrm{C}$ NMR, ${ }^{31} \mathrm{P}$ NMR and IR spectroscopic data matched that reported in the literature. ${ }^{5}$ 
$1 \mathrm{mmol}$ scale: Compound $\mathbf{1 9}$ was prepared according to a modified General Procedure A using a flame-dried $50 \mathrm{~mL}$ round-bottom flask, $\mathbf{P 3}$ (364 mg, $1.05 \mathrm{mmol}, 1.05$ equiv), SPhos (452 mg, 1.10 mmol, 1.10 equiv), 2,5-dioxopyrrolidin-1-yl 3-(4-bromophenyl)propanoate (326 mg, $1.00 \mathrm{mmol}$, 1.00 equiv), and cyclohexane $(10.0 \mathrm{~mL})$. The reaction mixture was allowed to stir at $60{ }^{\circ} \mathrm{C}$ for 16 $\mathrm{h}$. After allowing the reaction mixture to cool to room temperature, the flask was opened to air and the reaction solution was diluted with pentane $(20 \mathrm{~mL})$ to assist with precipitation. The precipitate was collected on a fritted funnel and washed with pentane $(30 \mathrm{~mL})$ and dried in vacuo on the Schlenk line overnight $(\sim 16 \mathrm{~h})$ to yield $19(803 \mathrm{mg}, 95 \%)$ as a pale-tan powder.

10 mmol scale: Compound $\mathbf{1 9}$ was prepared according to a modified General Procedure $\mathbf{A}$ using a flame-dried $500 \mathrm{~mL}$ round-bottom flask, P3 (3.642 g, $10.5 \mathrm{mmol}, 1.05$ equiv), SPhos (4.52 g, 11.0 mmol, 1.10 equiv), 2,5-dioxopyrrolidin-1-yl 3-(4-bromophenyl)propanoate (3.26 g, $10.0 \mathrm{mmol}$, 1.00 equiv), and cyclohexane $(100 \mathrm{~mL})$. The reaction mixture was allowed to stir at $60{ }^{\circ} \mathrm{C}$ for 16 $\mathrm{h}$. After allowing the reaction mixture to cool to room temperature, the flask was opened to air and the reaction solution was diluted with pentane $(200 \mathrm{~mL})$ to assist with precipitation. The precipitate was collected on a fritted funnel and washed with pentane $(300 \mathrm{~mL})$ and dried in vacuo on the Schlenk line overnight $(\sim 16 \mathrm{~h})$ to yield $19(8.34 \mathrm{~g}, 99 \%)$ as a pale-tan powder.

Note: Numerous new peaks in the ${ }^{1} H$ NMR spectrum can be seen in more concentrated samples of 19. Thus, ${ }^{1} H$ NMR spectra of both a dilute and a more concentrated sample of 19 are included.

${ }^{1} \mathbf{H}$ NMR $\left(500 \mathrm{MHz}, \mathrm{CDCl}_{3}\right) \delta 7.79(\mathrm{t}, J=8.4 \mathrm{~Hz}, 1 \mathrm{H}), 7.67-7.60(\mathrm{~m}, 1 \mathrm{H}), 7.47(\mathrm{tt}, J=7.2,1.6$ $\mathrm{Hz}, 1 \mathrm{H}), 7.44-7.37(\mathrm{~m}, 1 \mathrm{H}), 7.03(\mathrm{dd}, J=8.2,2.3 \mathrm{~Hz}, 2 \mathrm{H}), 6.87-6.81(\mathrm{~m}, 3 \mathrm{H}), 6.65(\mathrm{~d}, J=8.4$ $\mathrm{Hz}, 2 \mathrm{H}), 3.78(\mathrm{~s}, 6 \mathrm{H}), 2.92(\mathrm{dd}, J=9.5,6.4 \mathrm{~Hz}, 2 \mathrm{H}), 2.86-2.79(\mathrm{~m}, 6 \mathrm{H}), 2.23-2.12(\mathrm{~m}, 2 \mathrm{H})$, $1.87(\mathrm{~d}, J=13.7 \mathrm{~Hz}, 2 \mathrm{H}), 1.75(\mathrm{t}, J=13.7 \mathrm{~Hz}, 4 \mathrm{H}), 1.68-1.52(\mathrm{~m}, 9 \mathrm{H}), 1.31-1.03(\mathrm{~m}, 7 \mathrm{H})$, $0.56(\mathrm{q}, J=13.1 \mathrm{~Hz}, 2 \mathrm{H})$.

${ }^{13} \mathrm{C}$ NMR $\left(151 \mathrm{MHz}, \mathrm{CDCl}_{3}\right) \delta 169.1,169.1,168.1,168.0,160.4,144.0,143.9,139.3,137.2$, $137.1,135.7,135.5,135.2,134.2,133.7,132.7,132.3,131.7,131.6,131.3,131.2,131.2,129.2$, $128.6,127.0,126.7,126.7,126.0,125.7,125.6,108.0,107.9,106.1,55.9,34.1,33.9,33.1,33.1$, $30.4,29.9,29.8,27.9,27.9,27.5,27.4,27.3,27.3,27.0,27.0,26.8,26.6,25.8,25.5$.

${ }^{31} \mathbf{P}$ NMR $\left(162 \mathrm{MHz}, \mathrm{CD}_{2} \mathrm{Cl}_{2}\right) \delta 33.20$.

M.P. $163-165^{\circ} \mathrm{C}$ (dec.). Lit.: $163{ }^{\circ} \mathrm{C}$ (dec.)

IR (neat): 2930, 2847, 1812, 1786, 1739, 1587, 1471, 1447, 1428, 1362, 1282, 1244, 1199, 1106, $1059,1009,998,893,848,808,780,756,728,645 \mathrm{~cm}^{-1}$

(sSPhos)Pd[(4-C $\left.\left.\mathrm{O}_{2} \mathrm{C}_{6} \mathrm{~F}_{5}\right) \mathrm{Ph}\right] \mathrm{Br}(20)$

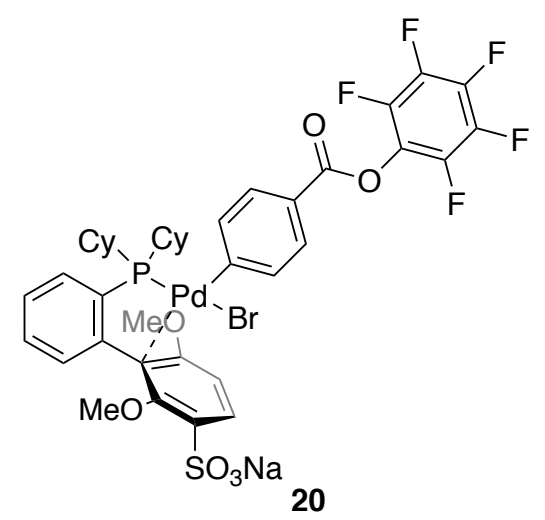

Compound $\mathbf{2 0}$ was prepared according to a modified General Procedure A. An oven-dried $24 \mathrm{~mL}$ reaction tube was equipped, while still hot, with a magnetic stir bar and a septum cap and was allowed to cool to room temperature under vacuum by piercing the septum with a needle connected to a dual manifold by a rubber hose. The cap was removed and the tube was charged with $\mathbf{P 3}$ (173 $\mathrm{mg}, 0.500 \mathrm{mmol}, 1$ equiv), sSPhos (269 mg, $0.525 \mathrm{mmol}, 1.05$ equiv), and perfluorophenyl 4-bromobenzoate (220 mg, $0.600 \mathrm{mmol}, 1.2$ equiv). The tube was brought into a $\mathrm{N}_{2}$-filled glovebox and 2MeTHF $(5.0 \mathrm{~mL})$ was added via syringe. The reaction tube 
was recapped, removed from the glove box, and placed under $\mathrm{N}_{2}$ by piercing the septum with a needle connected to a double manifold by a rubber hose. The reaction mixture was allowed to stir at $60{ }^{\circ} \mathrm{C}$ for $24 \mathrm{~h}$. At this time, the reaction tube was removed from the oil bath and the reaction mixture was allowed to cool to room temperature. The tube was opened to air and the reaction mixture was diluted with pentane $(15 \mathrm{~mL})$ to form an off-white precipitate. The precipitate was collected on a fritted funnel and washed with pentane $(25 \mathrm{~mL})$ and dried in vacuo on the Schlenk line overnight $(\sim 16 \mathrm{~h})$ to yield $\mathbf{2 0}(410 \mathrm{mg}, 83 \%)$ as an off-white powder.

Note: The use of dry 2-MeTHF is essential to minimize hydrolysis of the ester. Due to significant broadening of peaks in the ${ }^{1} \mathrm{H}$ and ${ }^{13} \mathrm{C}$ NMR spectra characteristic of SSPhos OACs, ${ }^{21}$ an LC trace is also provided.

${ }^{1} \mathbf{H}$ NMR $\left(600 \mathrm{MHz}, \mathrm{CD}_{2} \mathrm{Cl}_{2}\right)$ Complex spectrum, see below.

${ }^{13} \mathbf{C}$ NMR (151 MHz, $\mathrm{CD}_{2} \mathrm{Cl}_{2}$ ) Complex spectrum, see below.

${ }^{19}$ F NMR (565 MHz, $\left.\mathrm{CD}_{2} \mathrm{Cl}_{2}\right) \delta-152.46--155.88(\mathrm{~m}),-160.70,-164.62$.

${ }^{31} \mathbf{P}$ NMR $\left(202 \mathrm{MHz}, \mathrm{CD}_{2} \mathrm{Cl}_{2}\right) \delta 37.74,33.97$.

M.P. $207-210^{\circ} \mathrm{C}$ (dec.)

IR (neat): 3053, 2932, 2852, 1751, 1573, 1516, 1464, 1452, 1395, 1237, 1175, 1149, 1099, 1083, $1050,1035,1009,915,889,848,806,758,744,690,654,630,621,590 \mathrm{~cm}^{-1}$

EA: Calcd. for $\mathrm{C}_{39} \mathrm{H}_{38} \mathrm{BrF}_{5} \mathrm{NaO}_{7} \mathrm{PPdS}$ : C, 47.50; H, 3.88. Found: C, 47.23; H, 4.08.

\section{$\left(t\right.$-BuXPhos) Pd[ $\left[\left(4-\mathrm{CO}_{2} \mathrm{C}_{6} \mathrm{~F}_{5}\right) \mathrm{Ph}\right] \mathrm{Br}(21)$}

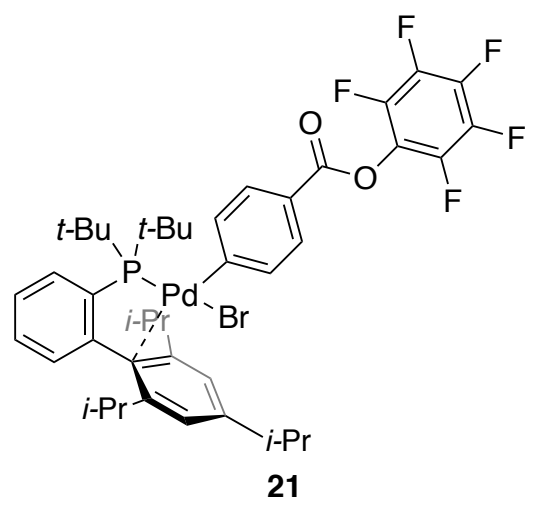

Compound 21 was prepared according to the General Procedure A using P3 (173 mg, $0.500 \mathrm{mmol}, 1$ equiv), $t$ BuXPhos (223 mg, 0.525 mmol, 1.05 equiv), perfluorophenyl 4-bromobenzoate $(220 \mathrm{mg}, 0.600 \mathrm{mmol}$, 1.2 equiv), and $n$-hexane $(5.0 \mathrm{~mL})$. The precipitate was collected on a fritted funnel to yield 21 (413 $\mathrm{mg}, 92 \%)$ as a pale-yellow powder.

${ }^{1} \mathbf{H}$ NMR $\left(600 \mathrm{MHz}, \mathrm{CD}_{2} \mathrm{Cl}_{2}\right) \delta 8.04-7.98(\mathrm{~m}, 1 \mathrm{H}), 7.60$ $(\mathrm{d}, J=8.4 \mathrm{~Hz}, 2 \mathrm{H}), 7.45-7.40(\mathrm{~m}, 4 \mathrm{H}), 7.13(\mathrm{~s}, 2 \mathrm{H}), 6.79$ $-6.74(\mathrm{~m}, 1 \mathrm{H}), 3.08$ (hept, $J=6.9 \mathrm{~Hz}, 1 \mathrm{H}), 2.57$ (hept, $J=$ $6.8 \mathrm{~Hz}, 2 \mathrm{H}), 1.60(\mathrm{~d}, J=6.8 \mathrm{~Hz}, 6 \mathrm{H}), 1.44(\mathrm{~s}, 9 \mathrm{H}), 1.42(\mathrm{~s}$, 9H), $1.39(\mathrm{~d}, J=6.9 \mathrm{~Hz}, 6 \mathrm{H}), 0.92(\mathrm{~d}, J=6.7 \mathrm{~Hz}, 6 \mathrm{H})$.

${ }^{13}$ C NMR $\left(151 \mathrm{MHz}, \mathrm{CD}_{2} \mathrm{Cl}_{2}\right) \delta 163.7,158.2,153.2,148.3,148.3,147.4,147.2,142.8,142.7$, 141.2 , 141.1, 141.0, 140.5, 140.3, 140.3, 139.2, 138.8, 137.6, 137.5, 137.4, 136.6, 136.3, 136.2, $135.7,135.0,134.9,130.7,130.7,126.8,126.3,126.3,125.4,124.6,124.6,121.6,40.2,40.1$, $34.8,31.9,31.8,31.8,25.6,25.0,24.8$.

${ }^{19}$ F NMR $\left(565 \mathrm{MHz}, \mathrm{CD}_{2} \mathrm{Cl}_{2}\right) \delta-154.04(\mathrm{~d}, J=17.3 \mathrm{~Hz}),-160.65--160.85(\mathrm{~m}),-164.46--$ $164.87(\mathrm{~m})$.

${ }^{31} \mathbf{P}$ NMR $\left(203 \mathrm{MHz}, \mathrm{CD}_{2} \mathrm{Cl}_{2}\right) \delta 52.14$.

M.P. $203-204{ }^{\circ} \mathrm{C}$ (dec.)

IR (neat): 2958, 2925, 2899, 2868, 1736, 1601, 1571, 1521, 1473, 1462, 1426, 1383, 1367, 1357 , $1312,1256,1173,1135,1050,1040,1005,992,974,884,829,765,758,742,732,619,595,574$ $\mathrm{cm}^{-1}$

EA: Calcd. for $\mathrm{C}_{42} \mathrm{H}_{49} \mathrm{BrF}_{5} \mathrm{O}_{2} \mathrm{PPd}$ : C, 56.17; H, 5.50. Found: C, 56.44; H, 5.71. 
$[(t-B u X P h o s) P d]_{2}(\operatorname{cod})(22)$

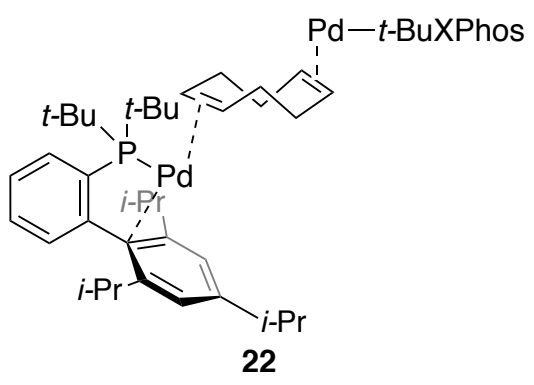

Compound 22 was prepared according to the General Procedure C using $\mathbf{P 3}$ (173 mg, $0.500 \mathrm{mmol}, 1$ equiv), $t$ BuXPhos $(223 \mathrm{mg}, 0.525 \mathrm{mmol}, 1.05$ equiv), and cyclohexane $(5.0 \mathrm{~mL})$. The precipitate was collected on a fritted funnel to yield $22(236 \mathrm{mg}, 81 \%)$ as a pale-green powder. The IR spectroscopic data matched that reported in the literature. ${ }^{22}$

Note: Compound 22 is insoluble in organic solvents and decomposes in chloroform, making direct solution-phase NMR spectroscopic characterization impossible. Previous publications only report IR spectroscopic characterization. ${ }^{22}$

M.P. $180-185^{\circ} \mathrm{C}($ dec. $)$

IR (neat): 2960, 2930, 2866, 1599, 1459, 1421, 1376, 1357, 1230, 1175, 1047, 924, 867, 851, 765, $747 \mathrm{~cm}^{-1}$

$\left[(\text { BrettPhos)Pd }]_{2}\right.$ (cod) (23)

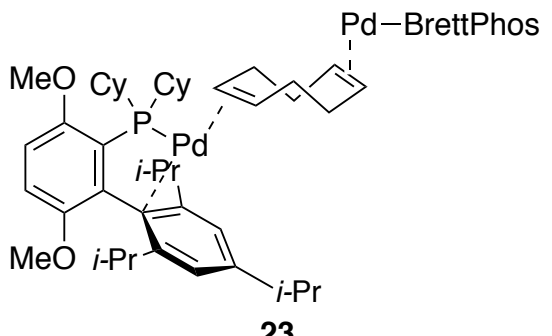

23
Compound 23 was prepared according to the General Procedure C using P3 (173 mg, $0.500 \mathrm{mmol}, 1$ equiv), BrettPhos (282 mg, $0.525 \mathrm{mmol}, 1.05$ equiv), and cyclohexane $(5.0 \mathrm{~mL})$. The precipitate was collected on a fritted funnel to yield $\mathbf{2 3}(281 \mathrm{mg}, 81 \%)$ as a yellow powder. Note: Compound $\mathbf{2 3}$ was determined to be $\left[(\text { BrettPhos)Pd }]_{2}(\mathrm{cod})\right.$ and not (BrettPhos)Pd(cod) by allowing 23 to react with 4-bromotoluene in THF- $\mathrm{d}_{8}$ and by allowing 23 to react with $\mathrm{CDCl}_{3}$; the ratio of $1,4-$

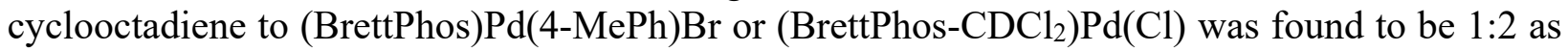
determined by ${ }^{1} \mathrm{H}$ NMR spectroscopy. These spectra are provided below.

Note: Compound $\mathbf{2 3}$ is insoluble in organic solvents and decomposes in chloroform, making direct solution-phase NMR spectroscopic characterization impossible. Previous publications only report IR spectroscopic characterization. ${ }^{22}$

M.P. 192-194 (dec.)

IR (neat): 2951, 2925, 2844, 2826, 1582, 1455, 1447, 1419, 1372, 1355, 1301, 1246, 1168, 1151, $1123,1085,1059,1012,926,889,867,846,794,761,739,709,664,614 \mathrm{~cm}^{-1}$

$\left[\left(\mathbf{X P h o s}_{)}\right) \mathrm{Pd}\right]_{2}(\mathbf{c o d})(\mathbf{2 4})$

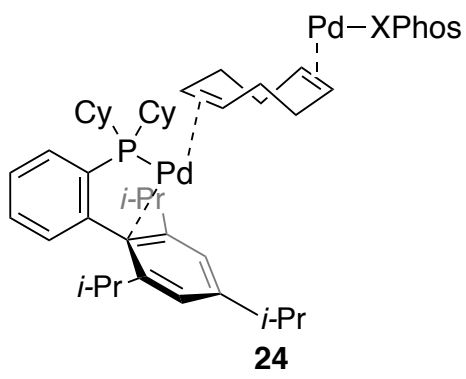

Compound $\mathbf{2 4}$ was prepared according to the General Procedure C using P3 (173 mg, $0.500 \mathrm{mmol}, 1$ equiv), XPhos (250 mg, $0.525 \mathrm{mmol}, 1.05$ equiv), and cyclohexane $(5.0 \mathrm{~mL})$. The precipitate was collected on a fritted funnel to yield $\mathbf{2 3}(279 \mathrm{mg}$, $88 \%)$ as a pale green powder.

Note: Compound $\mathbf{2 4}$ was determined to be $[(\mathrm{XPhos}) \mathrm{Pd}]_{2}(\operatorname{cod})$ and not (XPhos)Pd(cod) by allowing 24 to react with $4-$ bromotoluene in toluene- $\mathrm{d}_{8}$ and by allowing $\mathbf{2 4}$ to react with 
$\mathrm{CDCl}_{3}$; the ratio of 1,4 -cyclooctadiene to (XPhos) $\mathrm{Pd}(4-\mathrm{MePh}) \mathrm{Br}$ or $\left(\mathrm{XPhos}-\mathrm{CDCl}_{2}\right) \mathrm{Pd}(\mathrm{Cl})$ was found to be 1:2 as determined by ${ }^{1} \mathrm{H}$ NMR spectroscopy. These spectra are provided below.

Note: Compound 24 is insoluble in organic solvents and decomposes in chloroform, making direct solution-phase NMR spectroscopic characterization impossible.

M.P. $179-184^{\circ} \mathrm{C}($ dec. $)$

IR (neat): 2953, 2923, 2847, 1597, 1459, 1452, 1440, 1426, 1381, 1353, 1305, 1265, 1232, 1194 , $1099,1071,1050,993,929,879,870,851,761,747,730,718,668 \mathrm{~cm}^{-1}$

EA: Calcd. for $\mathrm{C}_{74} \mathrm{H}_{110} \mathrm{P}_{2} \mathrm{Pd}_{2}$ : C, 69.74; H, 8.70. Found: C, 70.03; H, 8.84.

\section{(XPhos)Pd( $\left.\mathrm{CH}_{2} \mathrm{CMe}_{2} \mathrm{C}_{6} \mathrm{H}_{4}\right)(25)$}

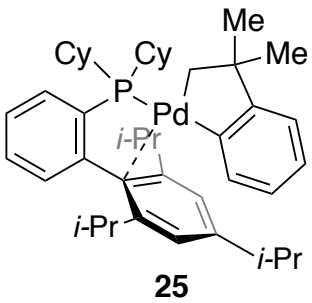

powder.

A $24 \mathrm{~mL}$ reaction tube equipped with a magnetic stir bar was charged with P3 (173 mg, $0.500 \mathrm{mmol}, 1.00$ equiv) and XPhos (239 mg, $0.500 \mathrm{mmol}$, 1.00 equiv). In air, $\mathrm{Et}_{2} \mathrm{O}(5.0 \mathrm{~mL})$ was added to the tube via syringe. The tube was capped and the mixture was allowed to stir in air for $1 \mathrm{~h}$. At this time, the cap was removed and the mixture was diluted with pentane (5 $\mathrm{mL})$ to assist with precipitation. The precipitate was collected on a fritted funnel and washed with pentane $(15 \mathrm{~mL})$ and dried in vacuo on the Schlenk line overnight $(\sim 16 \mathrm{~h})$ to yield $25(216 \mathrm{mg}, 60 \%)$ as an off-white

${ }^{1} \mathbf{H}$ NMR $\left(600 \mathrm{MHz}, \mathrm{CD}_{2} \mathrm{Cl}_{2}\right) \delta 7.75(\mathrm{ddd}, J=7.3,5.1,1.6 \mathrm{~Hz}, 1 \mathrm{H}), 7.42(\mathrm{td}, J=7.4,1.6 \mathrm{~Hz}$, $1 \mathrm{H}), 7.41-7.36(\mathrm{~m}, 1 \mathrm{H}), 7.15(\mathrm{~s}, 2 \mathrm{H}), 7.09$ (ddd, $J=7.4,4.0,1.4 \mathrm{~Hz}, 1 \mathrm{H}), 6.88$ (ddd, $J=7.6$, $3.5,1.6 \mathrm{~Hz}, 1 \mathrm{H}), 6.78(\mathrm{td}, J=7.3,1.4 \mathrm{~Hz}, 1 \mathrm{H}), 6.73(\mathrm{tt}, J=7.3,1.4 \mathrm{~Hz}, 1 \mathrm{H}), 6.62(\mathrm{dt}, J=7.3$, $1.4 \mathrm{~Hz}, 1 \mathrm{H}), 2.89$ (hept, $J=6.9 \mathrm{~Hz}, 1 \mathrm{H}), 2.47(\mathrm{~h}, J=6.8 \mathrm{~Hz}, 2 \mathrm{H}), 2.32-2.23(\mathrm{~m}, 2 \mathrm{H}), 2.18-$ $2.11(\mathrm{~m}, 2 \mathrm{H}), 1.86-1.76(\mathrm{~m}, 6 \mathrm{H}), 1.70(\mathrm{~d}, J=14.9 \mathrm{~Hz}, 4 \mathrm{H}), 1.60(\mathrm{qt}, J=13.2,4.0 \mathrm{~Hz}, 2 \mathrm{H}), 1.48$ $(\mathrm{d}, J=6.8 \mathrm{~Hz}, 6 \mathrm{H}), 1.36-1.23(\mathrm{~m}, 7 \mathrm{H}), 1.21(\mathrm{~m}, 7 \mathrm{H}), 1.14(\mathrm{~s}, 6 \mathrm{H}), 0.90(\mathrm{~d}, J=6.7 \mathrm{~Hz}, 6 \mathrm{H})$.

${ }^{13} \mathrm{C}$ NMR $\left(151 \mathrm{MHz}, \mathrm{CD}_{2} \mathrm{Cl}_{2}\right) \delta 166.7,151.1,148.4,148.2,146.8,137.1,137.0,135.7,135.6$, 133.6, 133.4, 133.3, 129.7, 129.7, 127.0, 127.0, 124.0, 123.6, 123.6, 123.1, 121.5, 120.7, 66.1, $49.3,35.5,35.4,34.6,33.0,33.0,31.6,30.4,30.4,29.1,28.0,28.0,27.9,27.9,26.7,25.8,24.5$, 24.1 .

${ }^{31} \mathbf{P}$ NMR $\left(162 \mathrm{MHz}, \mathrm{CD}_{2} \mathrm{Cl}_{2}\right) \delta 19.86$.

M.P. $126-129{ }^{\circ} \mathrm{C}$ (dec.)

IR (neat): 3055, 3043, 2946, 2930, 2918, 2849, 1608, 1582, 1568, 1554, 1464, 1443, 1424, 1381, 1360, 1348, 1324, 1312, 1293, 1267, 1258, 1241, 1196, 1182, 1170, 1161, 1123, 1102, 1085, 1078, 1073, 1052, 1043, 1016, 998, 936, 919, 886, 848, 815, 775, 765, 751, 742, 723, 647, $623 \mathrm{~cm}^{-1}$

EA: Calcd. for $\mathrm{C}_{43} \mathrm{H}_{61} \mathrm{PPd}$ : C, 72.20; H, 8.60. Found: C, 72.42; H, 8.72.

\section{(RuPhos)Pd( $\left.\mathrm{CH}_{2} \mathrm{CMe}_{2} \mathrm{C}_{6} \mathrm{H}_{4}\right)(26)$}

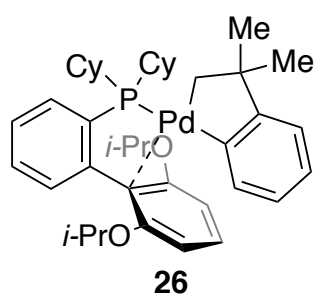

A $24 \mathrm{~mL}$ reaction tube was equipped with a magnetic stir bar and was charged with $\mathbf{P 3}$ (173 mg, $0.500 \mathrm{mmol}, 1.00$ equiv) and RuPhos (234 mg, $0.500 \mathrm{mmol}, 1.00$ equiv). In air, pentane $(5.0 \mathrm{~mL})$ was added to the tube via syringe. The tube was capped and the mixture was allowed to stir in air for $1 \mathrm{~h}$, over which time the white slurry dissolved to become an orange-colored translucent solution. After $1 \mathrm{~h}$, the cap was removed and the side of the tube was gently scratched with a glass stir rod, which 
caused the rapid formation of a white precipitate. The tube was recapped was returned and the slurry was allowed to stir for $5 \mathrm{~min}$. The cap was removed, and the precipitate was collected on a fritted funnel and washed with cold pentane $\left(0^{\circ} \mathrm{C}, 10 \mathrm{~mL}\right)$ and dried in vacuo on the Schlenk line overnight $(\sim 16 \mathrm{~h})$ to yield $\mathbf{2 6}(280 \mathrm{mg}, 80 \%)$ as a white powder.

${ }^{1} \mathbf{H}$ NMR $\left(500 \mathrm{MHz}, \mathrm{CD}_{2} \mathrm{Cl}_{2}\right) \delta 7.60(\mathrm{ddt}, J=7.7,5.4,2.2 \mathrm{~Hz}, 1 \mathrm{H}), 7.54(\mathrm{td}, J=6.1,2.4 \mathrm{~Hz}$, $1 \mathrm{H}), 7.43-7.35(\mathrm{~m}, 2 \mathrm{H}), 7.21-7.13(\mathrm{~m}, 2 \mathrm{H}), 6.94-6.85(\mathrm{~m}, 2 \mathrm{H}), 6.80-6.72(\mathrm{~m}, 3 \mathrm{H}), 4.55$ (hept, $J=6.0 \mathrm{~Hz}, 2 \mathrm{H}), 2.15-2.06(\mathrm{~m}, 4 \mathrm{H}), 1.80-1.70(\mathrm{~m}, 6 \mathrm{H}), 1.66(\mathrm{~d}, J=11.5 \mathrm{~Hz}, 4 \mathrm{H}), 1.51$ $-1.30(\mathrm{~m}, 6 \mathrm{H}), 1.28(\mathrm{~d}, J=6.0 \mathrm{~Hz}, 7 \mathrm{H}), 1.15(\mathrm{app} \mathrm{s}, 7 \mathrm{H}), 1.04(\mathrm{~d}, J=6.0 \mathrm{~Hz}, 6 \mathrm{H}), 0.90(\mathrm{t}, J=$ $7.1 \mathrm{~Hz}, 2 \mathrm{H})$.

${ }^{13} \mathrm{C}$ NMR $\left(126 \mathrm{MHz}, \mathrm{CD}_{2} \mathrm{Cl}_{2}\right) \delta 170.8,170.0,164.9,164.9,154.6,142.5,142.3,135.5,135.5$, $134.8,134.7,132.1,129.4,129.2,129.0,128.7,128.6,128.6,126.6,126.6,124.1,124.0,123.2$, $121.6,121.6,111.4,73.0,50.8,50.7,41.5,33.2,32.7,32.6,28.9,28.2,28.1,27.9,27.8,27.4$, 27.4, 26.7, 22.7, 21.6.

${ }^{31} \mathbf{P}$ NMR $\left(203 \mathrm{MHz}, \mathrm{CD}_{2} \mathrm{Cl}_{2}\right) \delta 19.54$.

M.P. $126-127^{\circ} \mathrm{C}$ (dec.)

IR (neat): 3046, 2970, 2923, 2913, 2847, 2795, 1594, 1578, 1462, 1447, 1426, 1381, 1367, 1346, 1327, 1291, 1265, 1227, 1194, 1180, 1168, 1137, 1116, 1104, 1087, 1050, 1019, 1002, 950, 936, $919,890,848,810,782,756,742,732,716,666,638 \mathrm{~cm}^{-1}$

HRMS (ESI) Calcd. For $\mathrm{C}_{40} \mathrm{H}_{56} \mathrm{O}_{2} \mathrm{PPd}(\mathrm{M}+\mathrm{H})^{+}$: 705.3047. Found: 705.3054 . 


\section{P3 as a Precatalyst for Pd-Catalyzed Cross-Coupling Reactions}

While $\mathrm{P} 3$ was used to access OACs previously utilized as precatalysts, we wanted to determine if P3 itself could act as a precatalyst for Pd catalysis. As P3 is bound to a labile ancillary ligand, it could function as a general precatalyst for cross-coupling reactions without requiring phosphine pre-ligation. Furthermore, its thermal activation eliminates the need for exogenous additives, and the nonpolar benzocyclobutene side product is easily removable by chromatography. The $\mathrm{C}-\mathrm{N}$ cross-coupling reaction of 3-bromoquinoline and morpholine with P3 as a precatalyst (Scheme $\mathrm{S} 1 \mathrm{~A}$ ) was used as a model reaction. In the presence of $1 \mathrm{~mol} \% \mathrm{P} 3$ and 2 mol\% RuPhos, 4-(quinolin3 -yl)morpholine 27 was quantitatively formed. While the use of the bulkier ligands BrettPhos, $t$ BuXPhos, and $t$-BuBrettPhos lead to diminished yield, the observation of product in each case proves that $\mathrm{P} 3$ is viable precatalyst in the presence of a variety of phosphine ligands. Product 27 was isolated in $92 \%$ yield when the reaction was performed on 1 mmol scale. Notably, utilizing the RuPhos pre-ligated complex 26 allowed for the synthesis of 27 in identical isolated yield (Scheme S1B). The use of a sample of $\mathbf{P 3}$ stored under ambient conditions for one year afforded 27 in similar yield.

\section{Scheme S1}

A. $^{a}$<smiles>Brc1cnc2ccccc2c1</smiles><smiles></smiles>
$+$<smiles>C1COCCN1</smiles><smiles>CC1(C)C[Pb](c2ccccc2)(P2C3CC4CC(C4)C2C3)c2ccccc21</smiles>

P3 (1 mol\%) 1.2 equiv

B. ${ }^{b}$<smiles>O=C(O)C1CCNCC1</smiles>
L (2 mol\%) $\mathrm{NaOt}$-Bu (1.2 equiv)<smiles>c1ccc2ncc(N3CCOCC3)cc2c1</smiles>

27 (NMR Yield) (Isolated Yield)

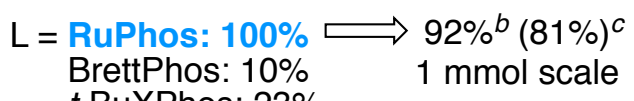
$t$-BuXPhos: 23\% $t$-BuBrettPhos: $6 \%$

1.2 equiv<smiles>CC1(C)C[Pb]([OH2+])c2ccccc21</smiles>

We investigated the use of $\mathbf{P 3}$ as a precatalyst by allowing it (1 mol\%) to react with 3bromoquinoline and morpholine in the presence of a variety of biarylphosphine ligands and $\mathrm{NaO}$ $\mathrm{Bu}$. An oven-dried $8 \mathrm{~mL}$ reaction tube (A) was equipped, while still hot, with a magnetic stir bar 
and septum cap and was allowed to cool to room temperature under vacuum by piercing the septum with a needle attached to a dual manifold by a rubber hose. The cap was removed and the tube was charged with $\mathrm{NaO} t$ - $\mathrm{Bu}$ (58 mg, $0.600 \mathrm{mmol}, 1.2$ equiv), 1,3,5-trimethoxybenzene (42 mg, 0.250 mmol, 0.5 equiv), and ligand ( $0.010 \mathrm{mmol}, 0.02$ equiv, $2 \mathrm{~mol} \%)$. A separate oven-dried $8 \mathrm{~mL}$ reaction tube (B, stock solution) was equipped, while still hot, with a magnetic stir bar and septum cap and was allowed to cool to room temperature under vacuum before being charged with P3 (17 $\mathrm{mg}, 0.050 \mathrm{mmol})$. The reaction tubes were brought into a nitrogen-filled glovebox. A was charged with 3-bromoquinoline $(67.9 \mu \mathrm{L}, 0.500 \mathrm{mmol}, 1.0$ equiv), morpholine (52.5 $\mu \mathrm{L}, 0.600 \mathrm{mmol}, 1.2$ equiv), and THF $(900 \mu \mathrm{L})$ via micropipettor. B was charged with THF $(1.00 \mathrm{~mL})$ via syringe. 100 $\mu \mathrm{L}$ of $\mathbf{B}(0.005 \mathrm{mmol} \mathbf{P 3}, 0.01$ equiv, $1 \mathrm{~mol} \%)$ was transferred to each vial $\mathbf{A}$ via syringe. $\mathbf{A}$ was capped with an unpunctured septum cap and removed from the glovebox. The top of the tube was covered with parafilm and the tube was placed in a pre-heated oil bath (bath temperature $=80{ }^{\circ} \mathrm{C}$ ). The reaction mixture was allowed to stir for $\sim 16 \mathrm{~h}$ at $80^{\circ} \mathrm{C}$. At this time the vials were removed from the oil bath and the reaction mixtures were allowed to cool to room temperature while remaining sealed. At this point, the reaction mixture in $\mathbf{A}$ was opened to air, diluted with $\mathrm{CH}_{2} \mathrm{Cl}_{2}$ ( $1 \mathrm{~mL}$ ), and filtered through a $\sim 2 \mathrm{~cm}$ Celite ${ }^{\circledR}$ plug in a Pasteur pipette. The Celite ${ }^{\circledR}$ plug was further washed with $\mathrm{CH}_{2} \mathrm{Cl}_{2}(5 \mathrm{~mL})$. The filtrate was concentrated in vacuo with the assistance of a rotary evaporator. The resulting residue was taken up in $\mathrm{CDCl}_{3}(1 \mathrm{~mL})$ and yield was determined by quantitative ${ }^{1} \mathrm{H}$ NMR spectroscopy against 1,3,5-trimethoxybenzene as an internal standard.

\section{4-(quinolin-3-yl)morpholine (27)}

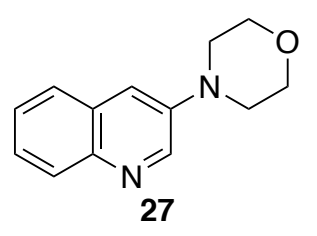

From P3: An oven-dried $16 \mathrm{~mL}$ reaction tube was equipped, while still hot, with a magnetic stir bar and septum cap and was allowed to cool to room temperature under vacuum by piercing the septum with a needle attached to a dual manifold by a rubber hose. The cap was removed and the tube was charged with $\mathrm{NaO} t$ - $\mathrm{Bu}$ (115 mg, $1.20 \mathrm{mmol}, 1.2$ equiv), RuPhos (9 mg, 0.02 mmol, 0.02 equiv, 2 mol\%), and $\mathbf{P 3}$ (3.5 mg, 0.01 mmol, 0.01 equiv, $1 \mathrm{~mol} \%)$. The tube was brought into a nitrogen-filled glovebox and charged with 3-bromoquinoline $(135.7 \mu \mathrm{L}, 1.00 \mathrm{mmol}, 1.0$ equiv), morpholine (105 $\mu \mathrm{L}, 1.20 \mathrm{mmol}, 1.2$ equiv), and THF (2.00 mL) via micropipettor. The tube was capped with an unpunctured septum cap and removed from the glovebox. The top of the tube was covered with parafilm and the tube was placed on a pre-heated oil bath (bath temperature $=80^{\circ} \mathrm{C}$ ). The reaction mixture was allowed to stir for $\sim 16 \mathrm{~h}$ at $80{ }^{\circ} \mathrm{C}$. At this time the vial was removed from the oil bath and the reaction mixture was allowed to cool to room temperature while remaining sealed. At this point, the vial was opened to air, diluted with $\mathrm{CH}_{2} \mathrm{Cl}_{2}(2 \mathrm{~mL})$, and filtered through a $\sim 1 \mathrm{~cm} \mathrm{Celite}{ }^{\circledR}$ plug in a 15 $\mathrm{mL}$ fritted funnel. The Celite ${ }^{\circledR}$ plug was further washed with $\mathrm{CH}_{2} \mathrm{Cl}_{2}(20 \mathrm{~mL})$. The filtrate was concentrated in vacuo with the assistance of a rotary evaporator. The crude material was purified by column chromatography (125 $\mathrm{mL} \mathrm{SiO}_{2}, 1: 3$ to 1:6 Hexane:EtOAc, 18 x $150 \mathrm{~mm}$ fractions, UV) and dried in vacuo on the Schlenk line overnight ( $16 \mathrm{~h})$ to yield $27(197 \mathrm{mg}, 92 \%)$ as a yellow powder. The ${ }^{1} \mathrm{H}$ NMR and ${ }^{13} \mathrm{C}$ NMR spectroscopic data matched that reported in the literature. ${ }^{23}$ Using P3 that was aged for 1 year in air at room temperature, 27 was obtained as a yellow powder (173 mg, 81\%).

Note: M.P. did not match literature reported value. However, 27 was confirmed to be pure by EA. 
${ }^{1}$ H NMR $\left(600 \mathrm{MHz}, \mathrm{CDCl}_{3}\right) \delta 8.78(\mathrm{~d}, J=2.9 \mathrm{~Hz}, 1 \mathrm{H}), 7.99(\mathrm{~d}, J=8.3 \mathrm{~Hz}, 1 \mathrm{H}), 7.67(\mathrm{~d}, J=8.1$ $\mathrm{Hz}, 1 \mathrm{H}), 7.54-7.49(\mathrm{~m}, 1 \mathrm{H}), 7.49-7.44(\mathrm{~m}, 1 \mathrm{H}), 7.33$ (d, $J=2.9 \mathrm{~Hz}, 1 \mathrm{H}), 3.94-3.90$ (m, 3H), $3.29-3.25(\mathrm{~m}, 3 \mathrm{H})$.

${ }^{13}$ C NMR $\left(151 \mathrm{MHz}, \mathrm{CDCl}_{3}\right) \delta 144.8,144.7,143.3,129.1,128.8,127.1,126.7,126.7,116.8$, $66.8,49.5$.

M.P. $94-95{ }^{\circ} \mathrm{C}$. Lit.: $84-86{ }^{\circ} \mathrm{C}^{23}$

EA: Calcd. for $\mathrm{C}_{13} \mathrm{H}_{14} \mathrm{~N}_{2} \mathrm{O}$ : C, 72.87; H, 6.59. Found: C, 72.90; H, 6.60 .

From 26: An oven-dried $16 \mathrm{~mL}$ reaction tube was equipped, while still hot, with a magnetic stir bar and septum cap and was allowed to cool to room temperature under vacuum by piercing the septum with a needle attached to a dual manifold by a rubber hose. The cap was removed and the tube was charged with $\mathrm{NaO} t-\mathrm{Bu}(115 \mathrm{mg}, 1.20 \mathrm{mmol}, 1.2$ equiv), and 26 ( $7.0 \mathrm{mg}, 0.01 \mathrm{mmol}, 0.01$ equiv, $1 \mathrm{~mol} \%$ ). The tube was brought into a $\mathrm{N}_{2}$-filled glovebox and charged with 3bromoquinoline (135.7 $\mu \mathrm{L}, 1.00 \mathrm{mmol}, 1.0$ equiv), morpholine (105 $\mu \mathrm{L}, 1.20 \mathrm{mmol}, 1.2$ equiv), and THF $(2.00 \mathrm{~mL})$ via micropipettor. The tube was capped with an unpunctured septum cap and removed from the glovebox. The top of the tube was covered with parafilm and the tube was placed in a pre-heated oil bath (bath temperature $=80^{\circ} \mathrm{C}$ ). The reaction mixture was allowed to stir for $\sim$ $16 \mathrm{~h}$ at $80^{\circ} \mathrm{C}$. At this time the vial was removed from the oil bath and the reaction mixture was allowed to cool to room temperature while still sealed. At this point, the tube was opened to air, the reaction mixture was diluted with $\mathrm{CH}_{2} \mathrm{Cl}_{2}(2 \mathrm{~mL})$, and filtered through a $\sim 1 \mathrm{~cm}$ Celite ${ }^{\circledR}$ plug in a $15 \mathrm{~mL}$ fritted funnel. The Celite ${ }^{\circledR}$ plug was further washed with $\mathrm{CH}_{2} \mathrm{Cl}_{2}(20 \mathrm{~mL})$. The filtrate was concentrated in vacuo with the assistance of a rotary evaporator. The crude material was purified by column chromatography $\left(125 \mathrm{~mL} \mathrm{SiO}_{2}, 1: 3\right.$ to $1: 6$ Hexane:EtOAc, $18 \times 150 \mathrm{~mm}$ fractions, UV) and dried in vacuo on the Schlenk line overnight ( $16 \mathrm{~h})$ to yield 27 (196 $\mathrm{mg}, 92 \%)$ as a yellow powder.

\section{Stability Studies}

Having demonstrated that $\mathbf{P 3}$ is stable in the solid state, we wanted to investigate if it shows similar stability in solution. To evaluate this stability, solutions of $\mathbf{P 3}$ were prepared in nine different deuterated solvents. P3 was not only found to be soluble in DMF- $\mathrm{d}_{7}$, dioxane-d8, THF$\mathrm{d}_{8}$, toluene- $\mathrm{d}_{8}$, chloroform- $\mathrm{d}$, and acetone- $\mathrm{d}_{6}$, it showed no evidence of decomposition after 24 hours in each solution. Although $\mathbf{P 3}$ was only sparingly soluble in methanol- $\mathrm{d}_{4}$, it again showed no signs of decomposition in solution. While $\mathbf{P 3}$ was found to be soluble in both DMSO- $\mathrm{d}_{6}$ and acetonitrile- $\mathrm{d}_{3}$, these solvents rapidly underwent reversible ligand exchange with the ligated cod, each favoring the solvate complex in $65 \%$ and $60 \%$, respectively. ${ }^{24}$ This equilibrium was reached within 15 minutes.

Finally, we analyzed the thermal stability of $\mathbf{P 3}$ in solution. When a solution of $\mathbf{P 3}$ in THF was heated to $60{ }^{\circ} \mathrm{C}$ for 2 hours, no spectroscopic evidence of decomposition was observed and only minimal decomposition, 7\%, was observed after 24 hours. In comparison, P1 immediately discolored upon heating to $60{ }^{\circ} \mathrm{C}$ and showed $42 \%$ decomposition after 2 hours. We found that both in the solid state and in the solution state, $\mathbf{P 3}$ is significantly more stable than $\mathbf{P 1}$ and allows for long-term storage and manipulation without decomposition. 


\section{Comparison of thermal stability of P3 vs. P1}

Two oven-dried $8 \mathrm{~mL}$ reaction tubes were equipped, while still hot, with magnetic stir bars and septum caps and was allowed to cool to room temperature under vacuum by piercing the septa with needles attached to a dual manifold by a rubber hose. The tubes were charged with 1,3,5trimethoxybenzene ( $8 \mathrm{mg}, 0.050 \mathrm{mmol}, 0.5$ equiv) and either $\mathbf{P 3}$ (35 mg, $0.100 \mathrm{mmol}, 1.0$ equiv) or P1 (39 mg, $0.100 \mathrm{mmol}, 1.0$ equiv). The tubes were capped, pierced with a needle connected to a Schlenk line, evacuated and backfilled with nitrogen (the evacuation/backfill process was repeated a total of three times). Under nitrogen, THF (anhydrous, $1.0 \mathrm{~mL}$ ) was added to each via syringe. The tubes were then placed in a preheated oil bath (bath temperature $=60{ }^{\circ} \mathrm{C}$ ). The reaction mixture was allowed to stir at $60^{\circ} \mathrm{C}$ for $2 \mathrm{~h}$. Within $1 \mathrm{~min}$ of being immersed in the oil bath, the solution containing P1 turned black in color. Over $2 \mathrm{~h}$, the solution containing P3 turned black in color, while significant mirroring (with, presumably, a Pd mirror) of the flask containing P1 was observed. After $2 \mathrm{~h}$, the flasks were removed from the oil bath and allowed to cool to room temperature under nitrogen. At room temperature, the reaction mixtures were transferred to $20 \mathrm{~mL}$ scintillation vials and the original flasks were washed with THF (stabilized, $1.5 \mathrm{~mL}$ ). The reaction mixtures were concentrated in vacuo with the assistance of a rotary evaporator. The resulting residues were taken up in $\mathrm{CDCl}_{3}(1 \mathrm{~mL})$, and the amount of decomposition was determined by quantitative ${ }^{1} \mathrm{H}$ NMR spectroscopy against 1,3,5-trimethoxybenzene as an internal standard.

P1: $40 \%$ decomposition after $2 \mathrm{~h}$ at $60{ }^{\circ} \mathrm{C}$

P3: $0 \%$ decomposition after $2 \mathrm{~h}$ at $60{ }^{\circ} \mathrm{C} .0 \%$ decomposition after $2 \mathrm{~h}$ at $60{ }^{\circ} \mathrm{C}$ in $n$-hexane. $6 \%$ decomposition after $24 \mathrm{~h}$ at $60{ }^{\circ} \mathrm{C}$ in THF. 


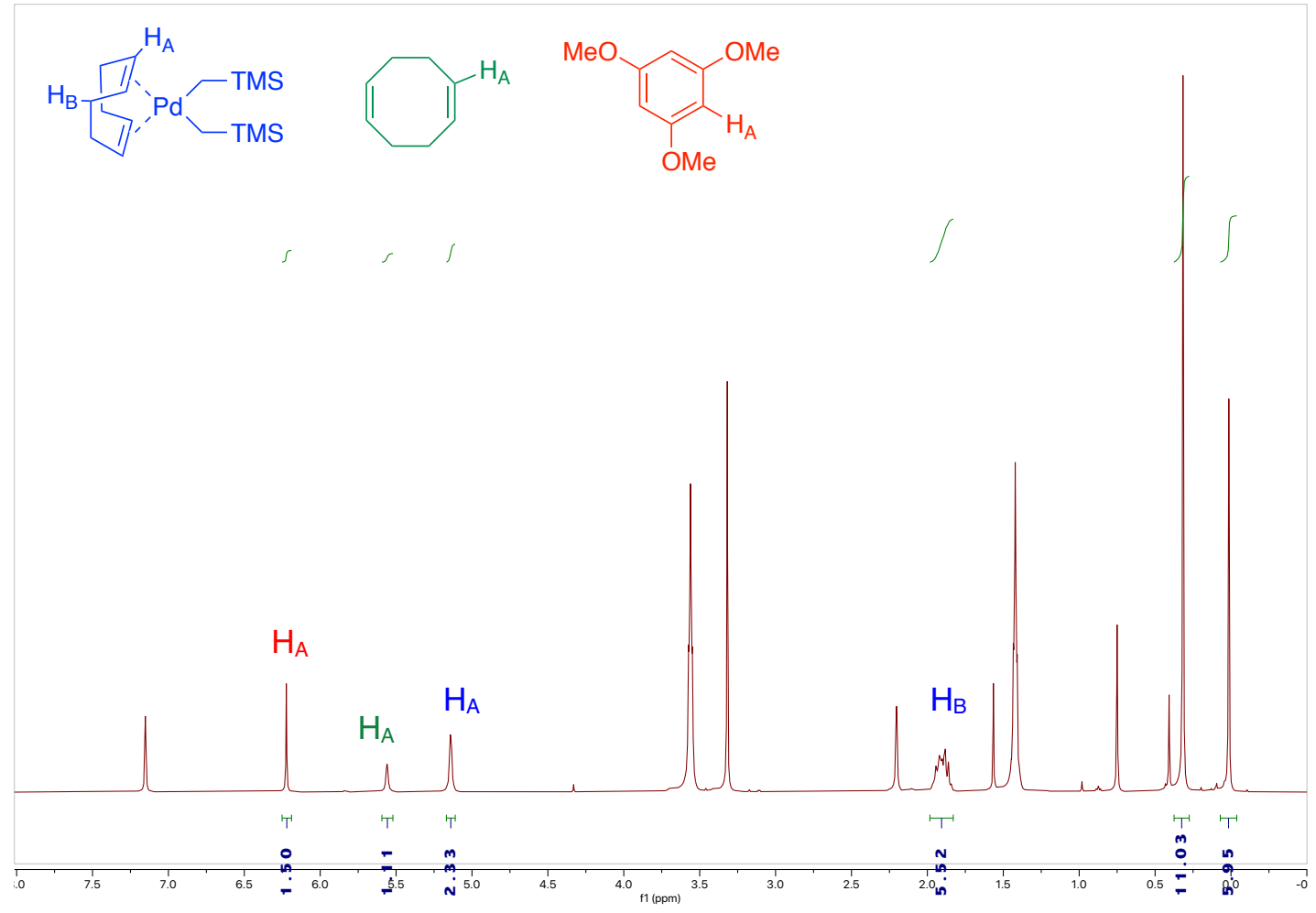

Figure S5: ${ }^{1} \mathrm{H}$ NMR spectrum of $\mathbf{P 1}$ after stirring for $2 \mathrm{~h}$ at $60{ }^{\circ} \mathrm{C}$ 

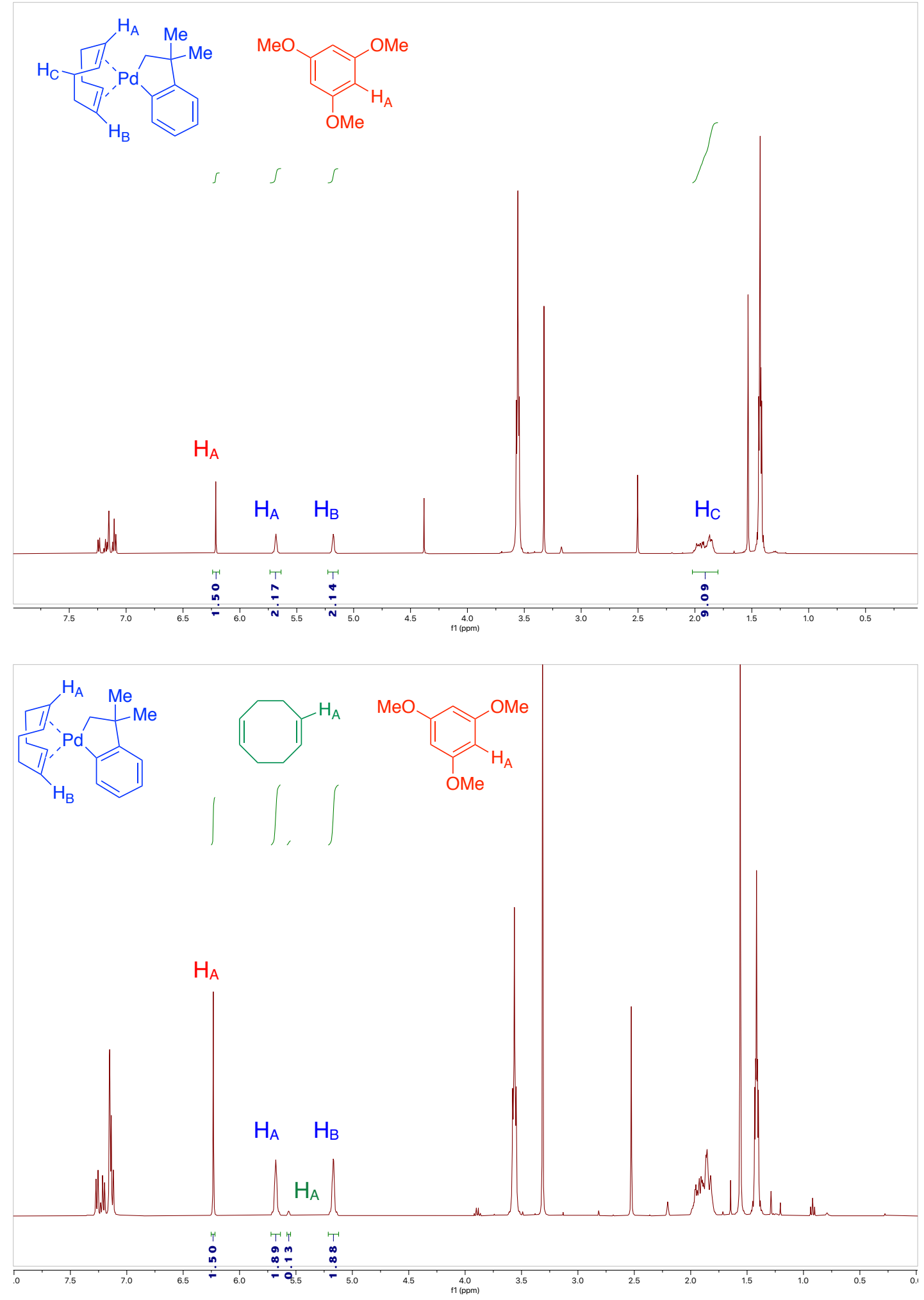

Figure S6: ${ }^{1} \mathrm{H}$ NMR spectra of $\mathbf{P 3}$ after stirring for $2 \mathrm{~h}$ (top) and $24 \mathrm{~h}$ (bottom) at $60{ }^{\circ} \mathrm{C}$ 


\section{Stability of P3 in various deuterated solvents}

Deuterated solvents used in this study and storage conditions:

$\mathrm{CDCl}_{3}$ : stored over activated $3 \AA$ molecular sieves

DMF- $\mathrm{d}_{7}$, DMSO- $\mathrm{d}_{6}$, THF- $\mathrm{d}_{8}$ : from ampules, opened immediately before use

Dioxane- $\mathrm{d}_{8}$, toluene- $\mathrm{d}_{8}$, methanol- $\mathrm{d}_{4}$, acetonitrile- $\mathrm{d}_{3}$, acetone- $\mathrm{d}_{6}$ : used from the bottle without any purification.

Nine different 1 dram vials were charged with $\mathbf{P 3}(17 \mathrm{mg}, 0.050 \mathrm{mmol})$ and 1,3,5trimethoxybenzene $(8 \mathrm{mg}, 0.050 \mathrm{mmol})$. In air, each vial was charged with a different deuterated solvent $(750 \mu \mathrm{L})$ before being transferred to an NMR tube. P3 was completely dissolved in all solvents except for acetonitrile- $\mathrm{d}_{3}$, in which it was moderately soluble, and methanol- $\mathrm{d}_{4}$, in which it was sparingly soluble. Each NMR tube was capped and sealed with electrical tape. Each mixture was studied by ${ }^{1} \mathrm{H}$ NMR spectroscopic analysis at three time points: $0 \mathrm{~h}, 2 \mathrm{~h}$, and $24 \mathrm{~h}$ after the preparation of the solution. In DMSO- $\mathrm{d}_{6}$ and acetonitrile- $\mathrm{d}_{3}$, partial exchange of cod with the solvent is observed. The exchange produces an equilibrium mixture of (cod) $\mathrm{Pd}\left(\mathrm{CH}_{2} \mathrm{CMe}_{2} \mathrm{C}_{6} \mathrm{H}_{4}\right)$ and $(\mathrm{S})_{2} \mathrm{Pd}\left(\mathrm{CH}_{2} \mathrm{CMe}_{2} \mathrm{C}_{6} \mathrm{H}_{4}\right)(\mathrm{S}=$ solvent $)$ that was observed at the $0 \mathrm{~h}$ time point and did not change after $24 \mathrm{~h}$. In all cases, there was no evidence of any decomposition after $24 \mathrm{~h}$ in solution. Shown below are the ${ }^{1} \mathrm{H}$ NMR spectra at $24 \mathrm{~h}$.

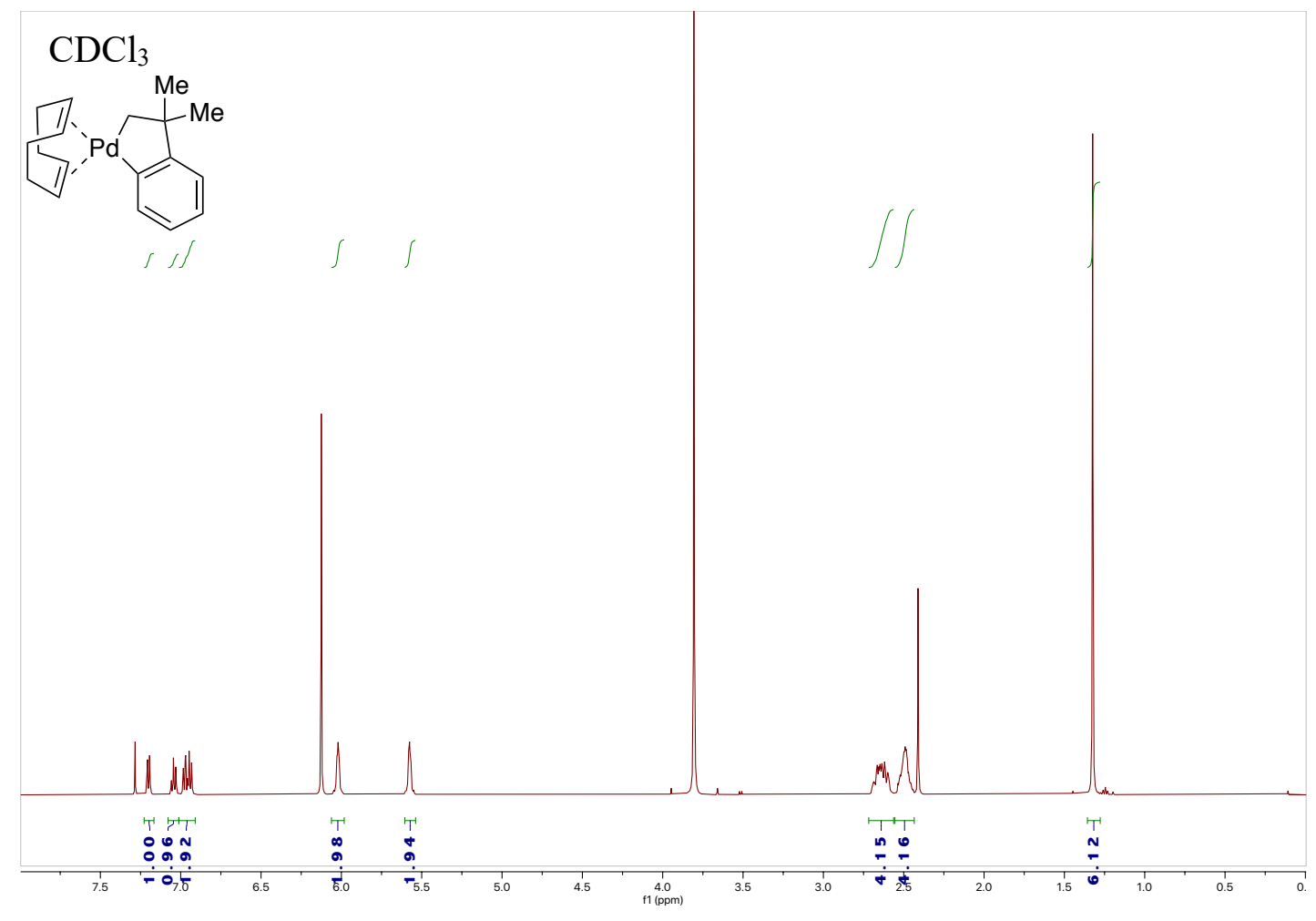




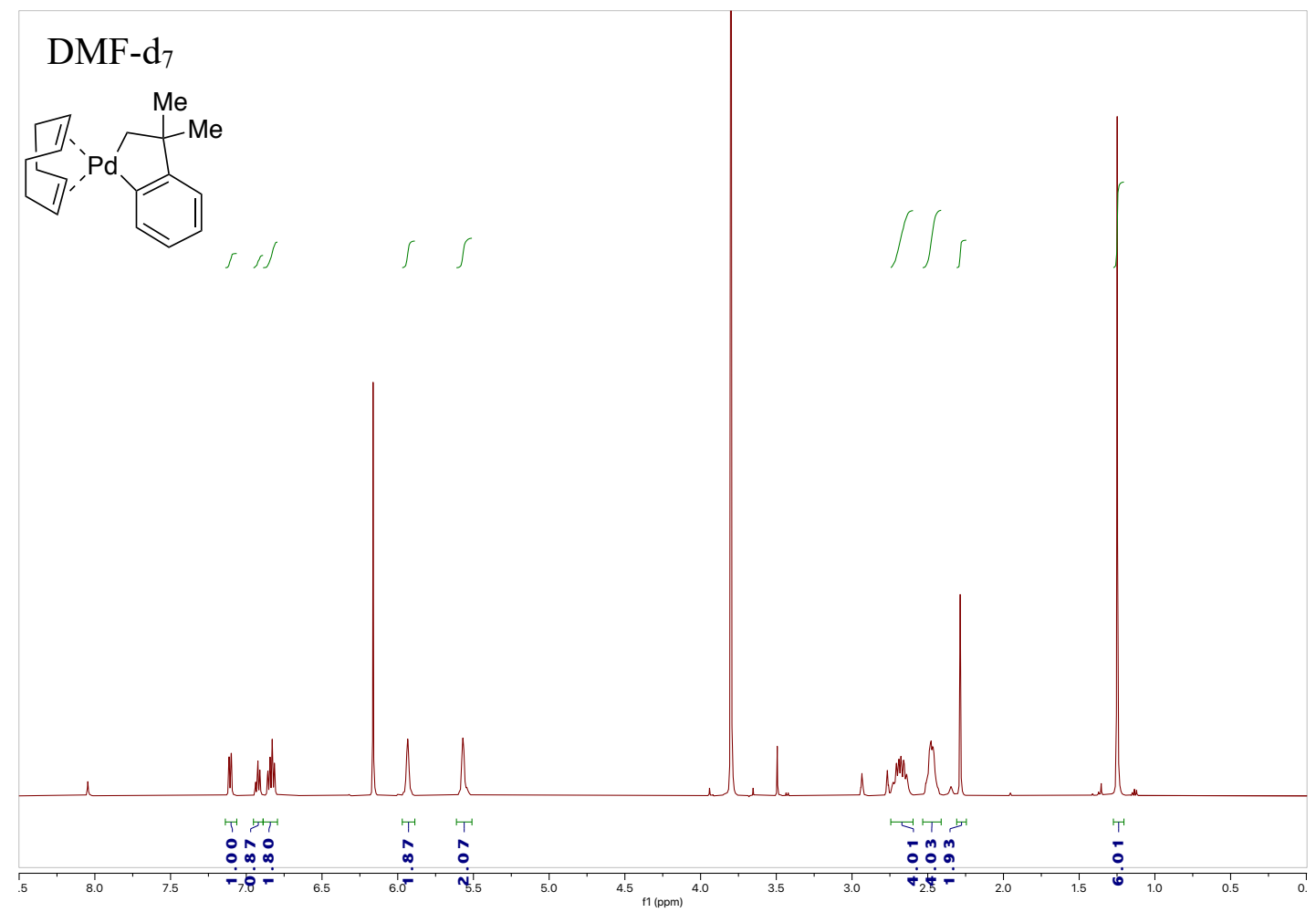

Toluene-d 8

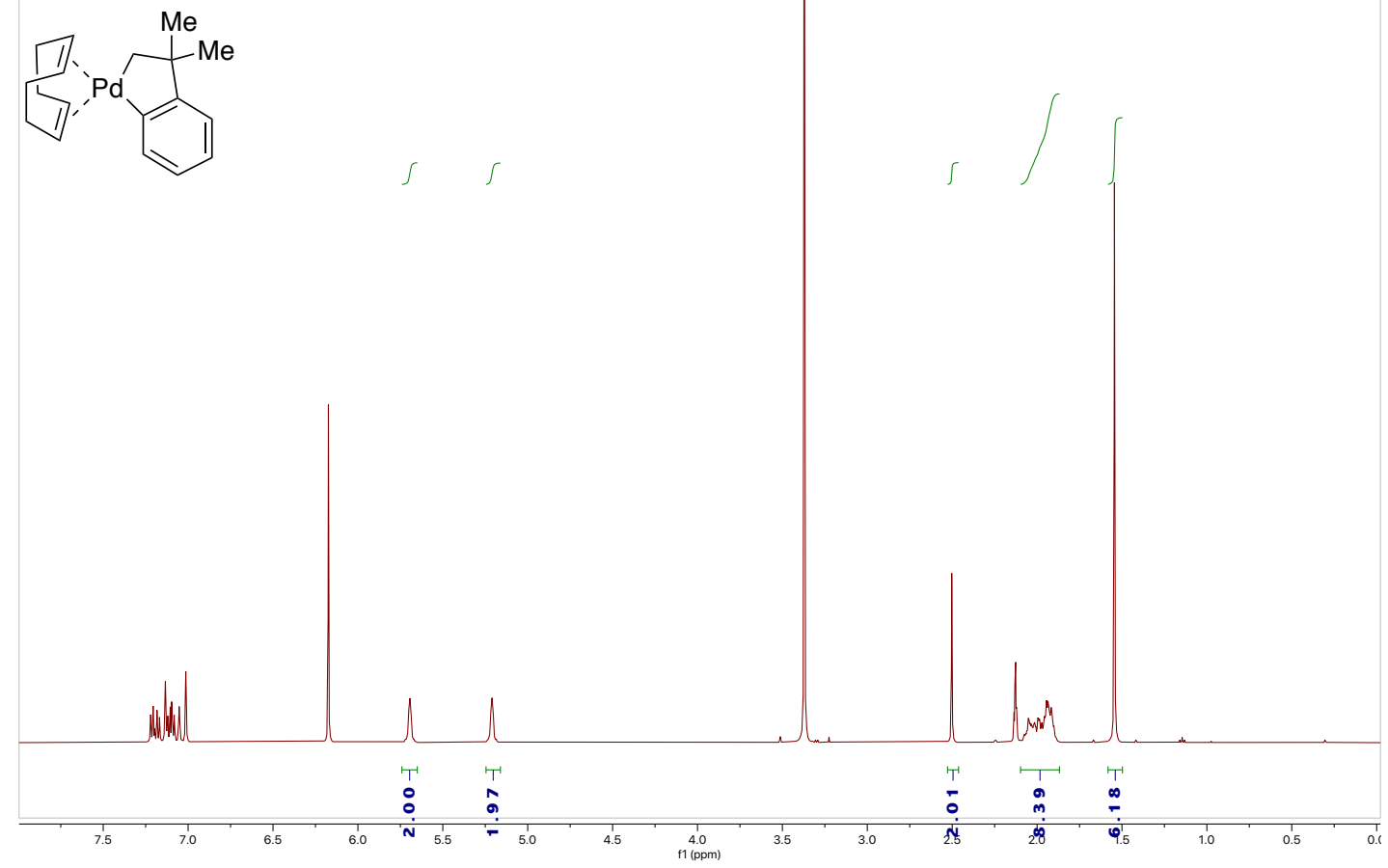



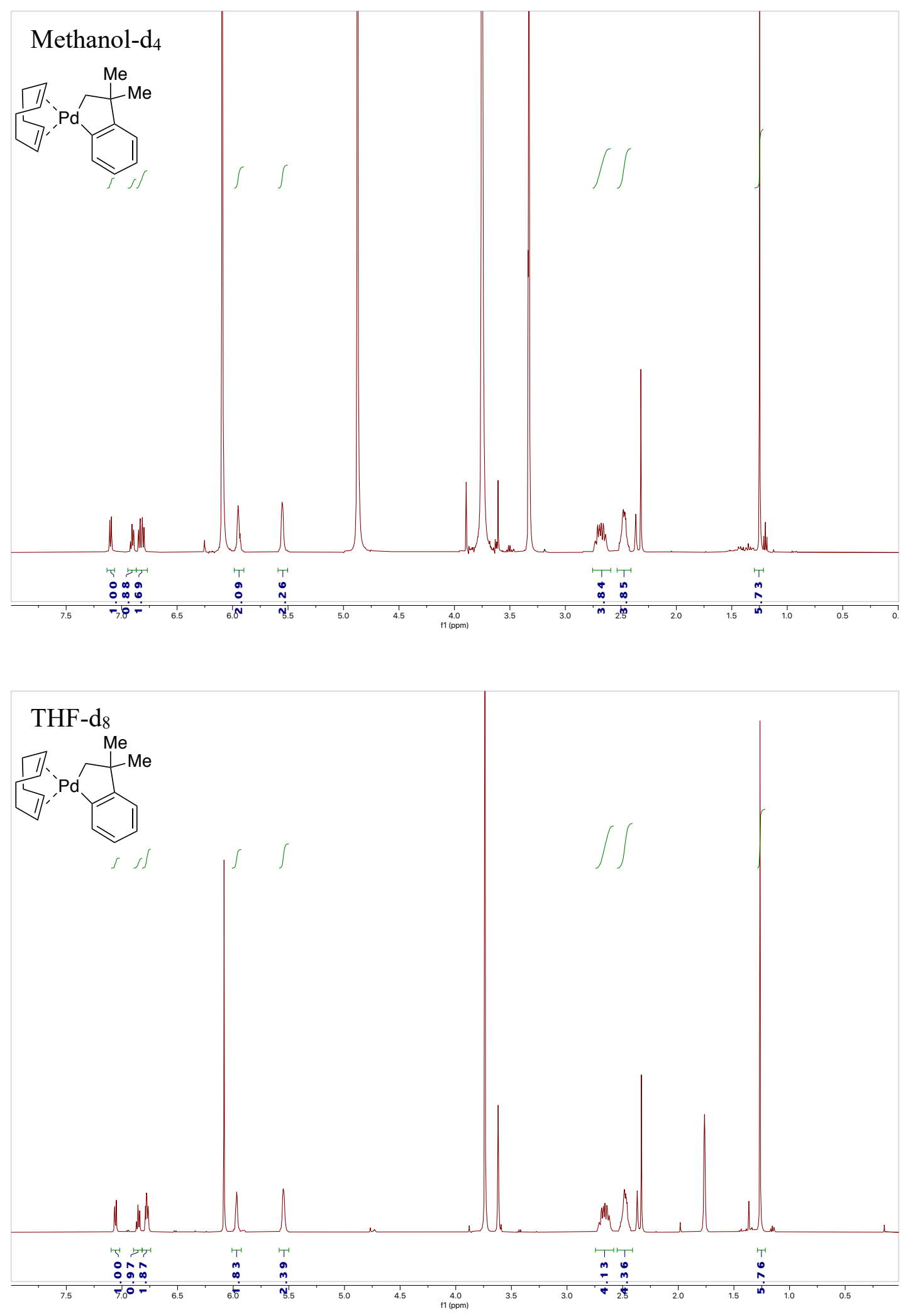

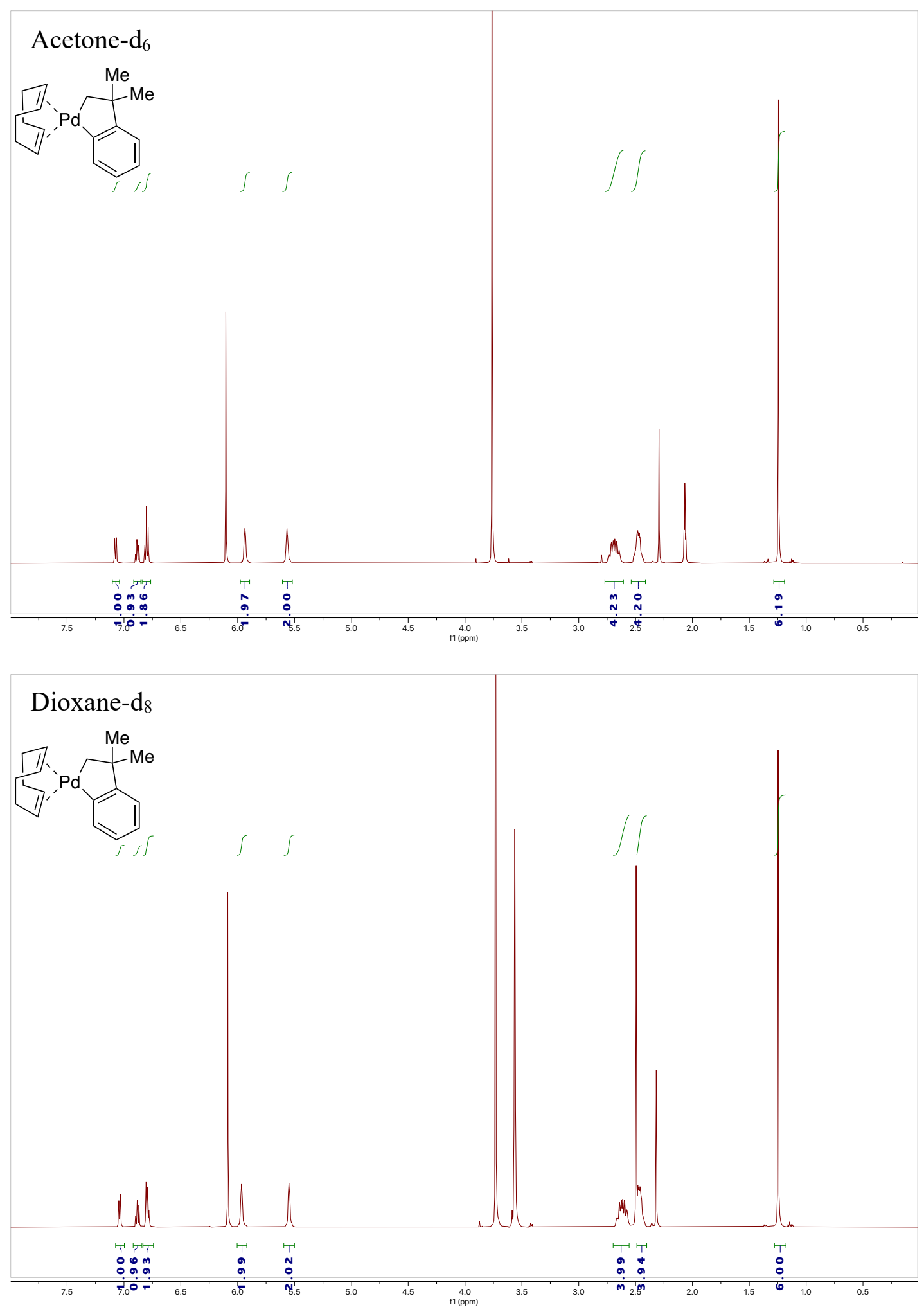

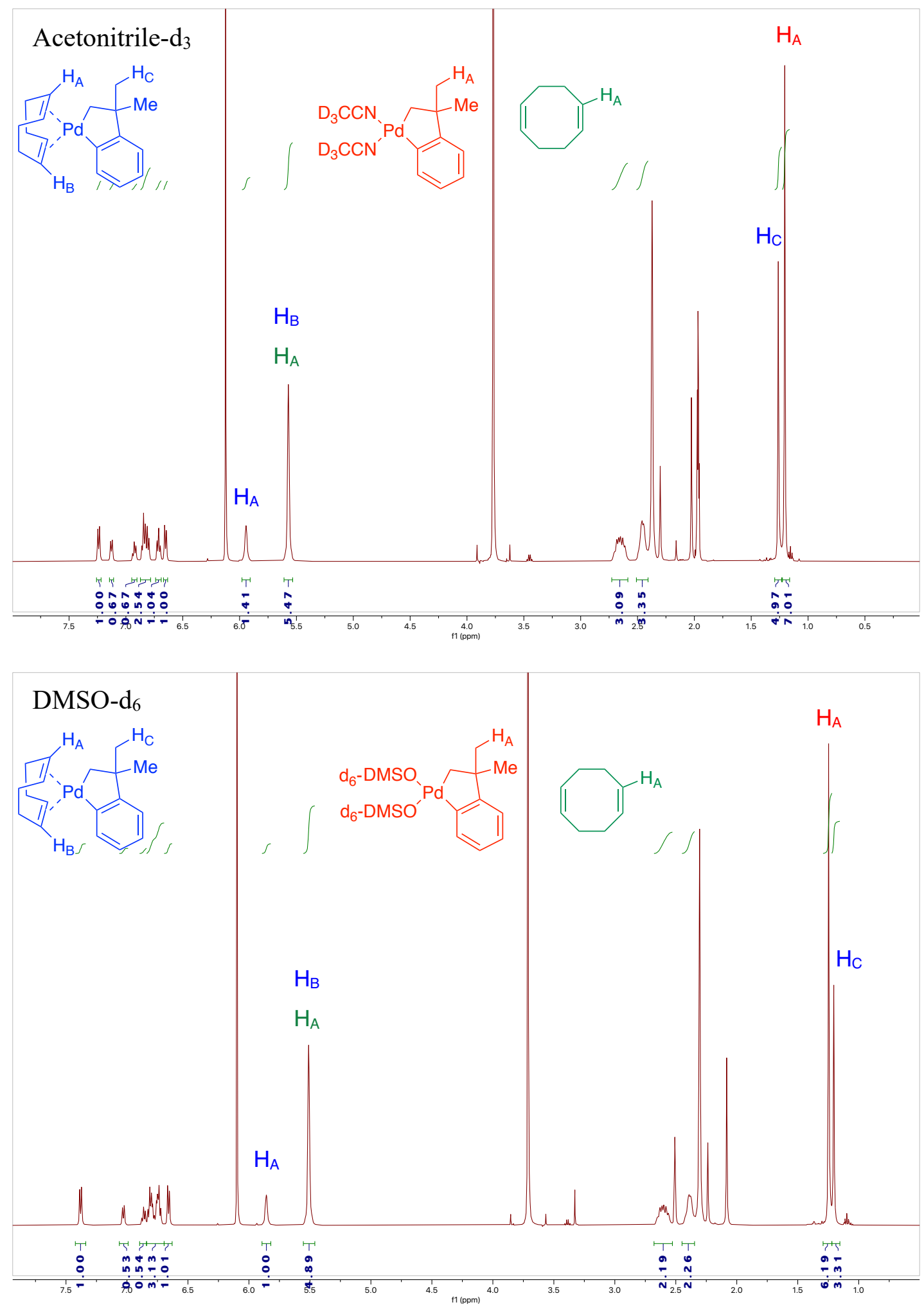

Figure S7: ${ }^{1} \mathrm{H}$ NMR spectra of $\mathbf{P 3}$ in various deuterated solvents, aged $24 \mathrm{~h}$. 


\section{References}

1. Fulmer, G. R.; Miller, A. J. M.; Sherden, M. H.; Gottlieb, H. E.; Nudelman, A.; Stoltz, B. M.; Bercaw, J. E.; Goldberg, K. I. NMR chemical shifts of trace impurities: common laboratory solvents, organics, and gases in deuterated solvents relevant to the organometallic chemist. Organometallics 2010, 29, 2176-2179.

2. Lovinger, G. J.; Aparece, M. D.; Morken, J. P. Pd-Catalyzed Conjunctive Cross-Coupling between Grignard-Derived Boron "Ate" Complexes and $\mathrm{C}\left(\mathrm{sp}^{2}\right)$ Halides or Triflates: NaOTf as a Grignard Activator and Halide Scavenger. J. Am. Chem. Soc. 2017, 139, 3153-3160.

3. Lee, H. G.; Milner, P. J.; Buchwald, S. L. An Improved Catalyst System for the Pd-Catalyzed Fluorination of (Hetero)Aryl Triflates. Org. Lett. 2013, 15, 5602-5605.

4. Ingoglia, B. T.; Buchwald, S. L. Oxidative Addition Complexes as Precatalysts for CrossCoupling Reactions Requiring Extremely Bulky Biarylphosphine Ligands. Org. Lett. 2017, 19, 2853-2856.

5. Dhanjee, H. H.; Buslov, I.; Windsor, I. W.; Raines, R. T.; Pentelute, B. L.; Buchwald, S. L. Palladium-Protein Oxidative Addition Complexes by Amine-Selective Acylation. J. Am. Chem. Soc. 2020, 142, 21237-21242.

6. Bao, Y.-S.; Zhaorigetu, B.; Agula, B.; Baiyan, M.; Jia, M. Aminolysis of Aryl Esters Using Tertiary Amine as Amino Donor via $\mathrm{C}-\mathrm{O}$ and $\mathrm{C}-\mathrm{N}$ Bond Activations. J. Org. Chem. 2014, 79, 803-808.

7. Sather, A. C.; Lee, H. G.; De La Rosa, V. Y.; Yang, Y.; Müller, P.; Buchwald, S. L. A Fluorinated Ligand Enables Room-Temperature and Regioselective Pd-Catalyzed Fluorination of Aryl Triflates and Bromides. J. Am. Chem. Soc. 2015, 137, 13433-13438.

8. Anderson, K. W.; Buchwald, S. L. General Catalysts for the Suzuki-Miyaura and Sonogashira Coupling Reactions of Aryl Chlorides and for the Coupling of Challenging Substrate Combinations in Water. Angew. Chem. Int. Ed. 2005, 44, 6173-6177.

9. Han, C.; Buchwald, S. L. Negishi Coupling of Secondary Alkylzinc Halides with Aryl Bromides and Chlorides. J. Am. Chem. Soc. 2009, 131, 7532-7533.

10. McCann, S. D.; Reichert, E. C.; Arrechea, P. L.; Buchwald, S. L. Development of an Aryl Amination Catalyst with Broad Substrate Scope Guided by Consideration of Catalyst Stability. $J$. Am. Chem. Soc. 2020, 142, 15027-15037.

11. Zhang, H.; Ruiz-Castillo, P.; Buchwald, S. L. Palladium-Catalyzed C-O Cross-Coupling of Primary Alcohols. Org. Lett. 2018, 20, 1580-1583.

12. Peng, K.; Mawamba, V.; Schulz, E.; Löhr, M.; Hagemann, C.; Schatzschneider, U. iClick Reactions of Square-Planar Palladium(II) and Platinum(II) Azido Complexes with Electron-Poor Alkynes: Metal-Dependent Preference for N1 vs N2 Triazolate Coordination and Kinetic Studies with ${ }^{1} \mathrm{H}$ and ${ }^{19} \mathrm{~F}$ NMR Spectroscopy. Inorg. Chem. 2019, 58, 11508-11521.

13. Cámpora, J.; López, J. A.; Palma, P.; del Rio, D.; Carmona, E.; Valerga, P.; Graiff, C.; Tripicchio, A. Synthesis and Insertion of the Cyclometalated Palladium-Alkyl Complexes $\mathrm{Pd}\left(\mathrm{CH}_{2} \mathrm{CMe}_{2}-O-\mathrm{C}_{6} \mathrm{H}_{4}\right) \mathrm{L}_{2}$. Observation of a Pentacoordinated Intermediate in the Insertion of $\mathrm{SO}_{2}$. Inorg. Chem. 2001, 40, 4116-4126.

14. McAtee; J. R.; Martin, S. E. S.; Ahneman, D. T.; Johnson, K. A.; Watson, D. A. Preparation of Allyl and Vinyl Silanes by the Palladium-Catalyzed Silylation of Terminal Olefins: A SilylHeck Reaction. Angew. Chem. Int. Ed. 2012, 51, 3663-3667.

15. López, G.; Garcia, G.; Santana, M. D.; Sánchez, G.; Ruiz, J.; Hermoso, J. A.; Vegas, A.; Martínez-Ripoll, M. Synthesis and structural study of neutral mononuclear and anionic binuclear 
2,4,6-trifluorophenyl derivatives of palladium(II). Crystal structure of $\left[\mathrm{P}\left(\mathrm{CH}_{2} \mathrm{Ph}\right) \mathrm{Ph}_{3}\right]_{2}\left[\left(\mathrm{C}_{6} \mathrm{~F}_{3} \mathrm{H}_{2}\right)_{2} \mathrm{Pd}(\mu-\mathrm{SCN})(\mu-\mathrm{NCS}) \mathrm{Pd}\left(\mathrm{C}_{6} \mathrm{~F}_{3} \mathrm{H}_{2}\right)_{2}\right]$. J. Chem. Soc., Dalton Trans. 1990, $1621-1626$.

16. Cho, E. J.; Senecal, T. D.; Kinzel, T.; Zhang, Y.; Watson, D. A.; Buchwald, S. L. The Palladium-Catalyzed Trifluoromethylation of Aryl Chlorides. Science 2010, 328, 1679-1681.

17. Uehling, M. R.; King, R. P.; Krska, S. W.; Cernak, T.; Buchwald, S. L. Pharmaceutical diversification via palladium oxidative addition complexes. Science 2019, 363, 405-408.

18. Vinogradova, E. V.; Zhang, C.; Spokoyny, A. M.; Pentelute, B. L.; Buchwald, S. L. Organometallic palladium reagents for cysteine bioconjugation. Nature 2015, 526, 687-691.

19. Rojas, A. J.; Zhang, C.; Vinogradova, E. V.; Buchwald, N. H.; Reilly, J.; Pentelute, B. L.; Buchwald, S. L. Divergent unprotected peptide macrocyclisation by palladium-mediated cysteine arylation. Chem. Sci. 2017, 8, 4257-4263.

20. Zhao, W.; Lee, H. G.; Buchwald, S. L.; Hooker, J. M. Direct ${ }^{11} \mathrm{CN}-$ Labeling of Unprotected Peptides via Palladium-Mediated Sequential Cross-Coupling Reactions. J. Am. Chem. Soc. 2017, 139, 7152-7155.

21. Rojas, A. J.; Pentelute, B. L.; Buchwald, S. L. Water-Soluble Palladium Reagents for Cysteine S-Arylation under Ambient Aqueous Conditions. Org. Lett. 2017, 19, 4263-4266.

22. Lee, H. G.; Milner, P. J.; Colvin, M. T.; Andreas, L.; Buchwald, S. L. Structure and reactivity of $\left[(\mathrm{L} \cdot \mathrm{Pd})_{n} \cdot(1,5\right.$-cyclooctadiene $\left.)\right](n=1-2)$ complexes bearing biaryl phosphine ligands. Inorg. Chim. Acta. 2014, 422, 188-192.

23. Malapit, C. A.; Borrell, M.; Milbauer, M. W.; Brigham, C. E.; Sanford, M. S. Nickel-Catalyzed Decarbonylative Amination of Carboxylic Acid Esters. J. Am. Chem. Soc. 2020, 142, 5918-5923. 24. Pd OACs are rarely prepared in acetonitrile or dimethylsulfoxide. However, we determined that both $\mathbf{4}$ and $\mathbf{2 6}$ could be generated in these solvents. 


\section{Copies of NMR Spectra}


(cod)PdCl 2 (S1)

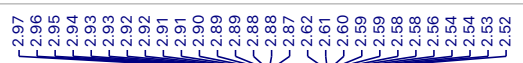

${ }^{1} \mathrm{H}$ NMR (500 MHz, $\left.\mathrm{CDCl}_{3}\right)$

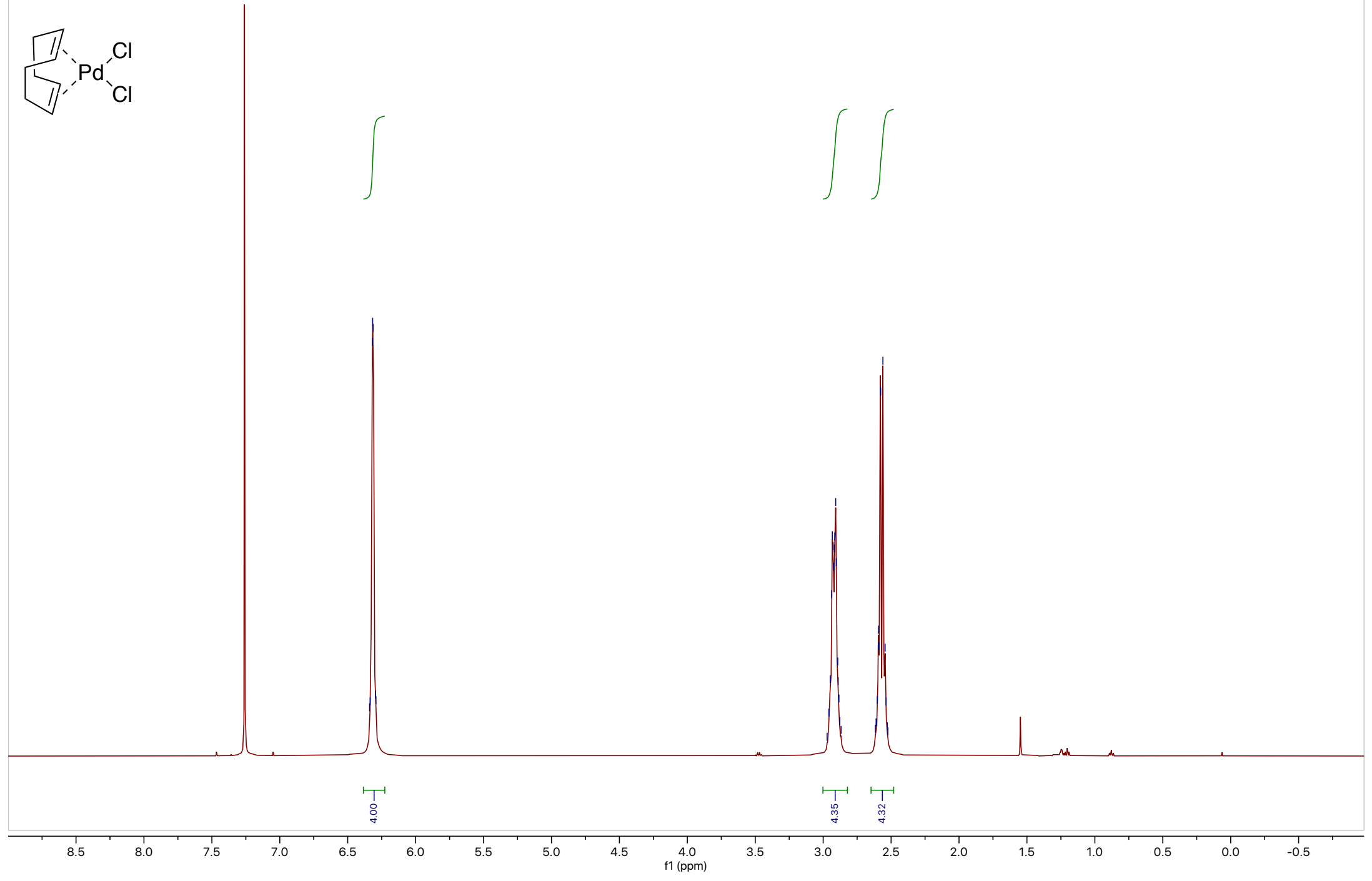


(cod)PdCl $\mathbf{P}_{2}$ (S1)

${ }^{13} \mathrm{C}$ NMR $\left(126 \mathrm{MHz}, \mathrm{CDCl}_{3}\right)$

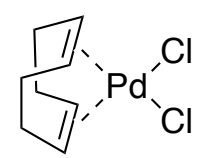

$\stackrel{\infty}{\stackrel{\infty}{\sigma}}$

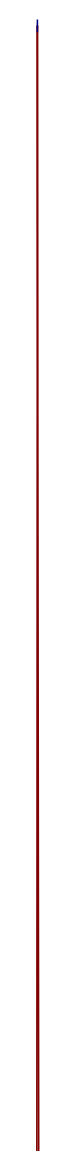

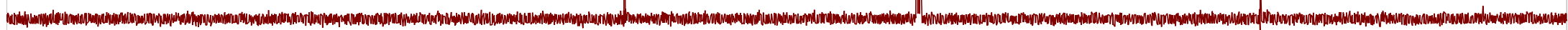

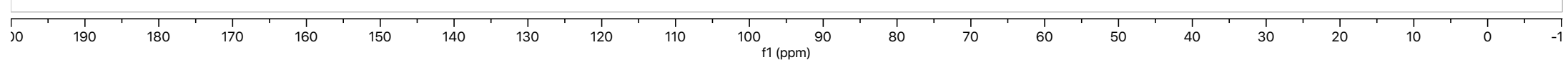




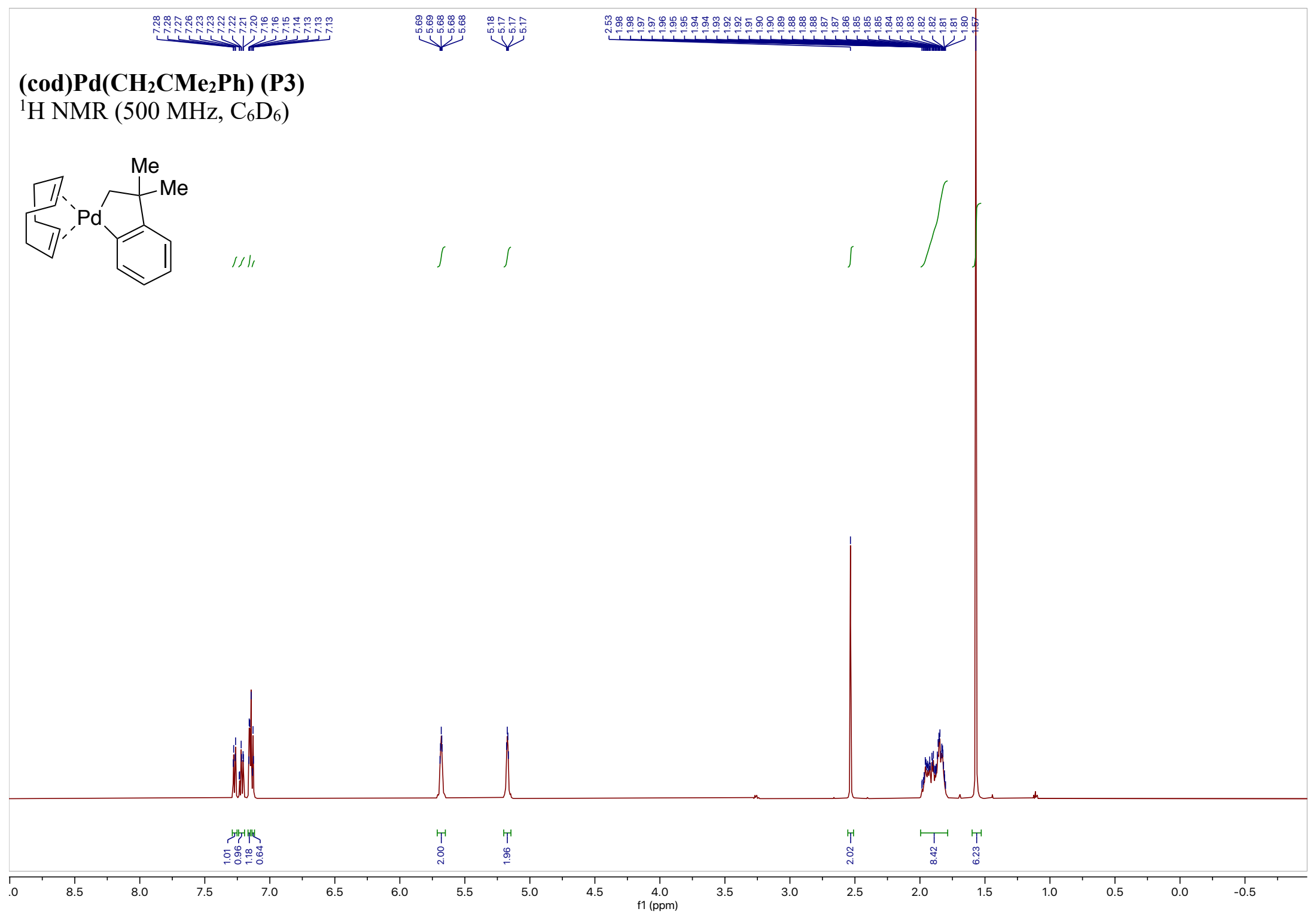




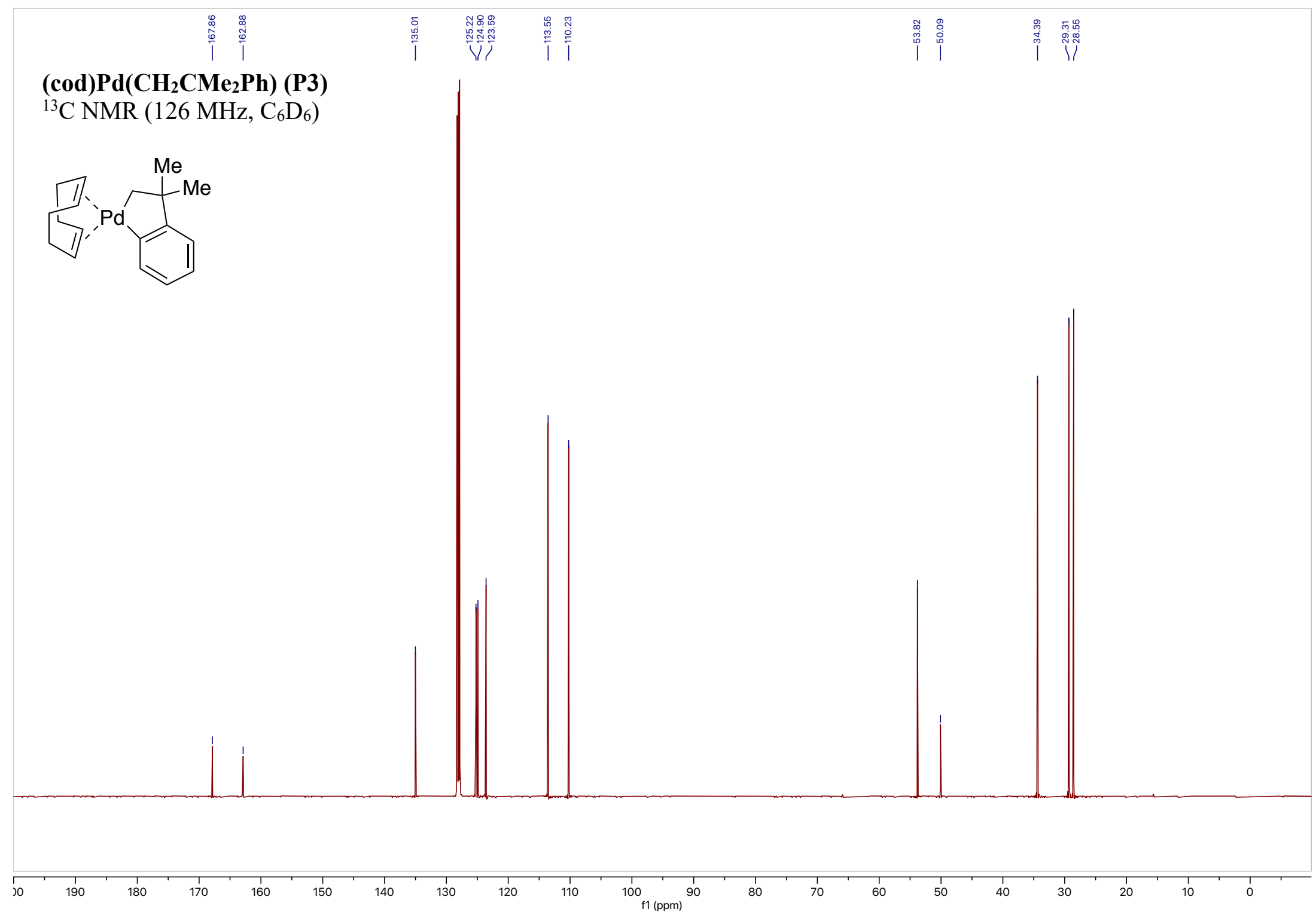


(cod)Pd(CH2 $\mathrm{CHS}_{2}$ (P1)

${ }^{1} \mathrm{H}$ NMR $\left(600 \mathrm{MHz}, \mathrm{C}_{6} \mathrm{D}_{6}\right)$

$$
\text { 1. }
$$

踥

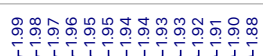
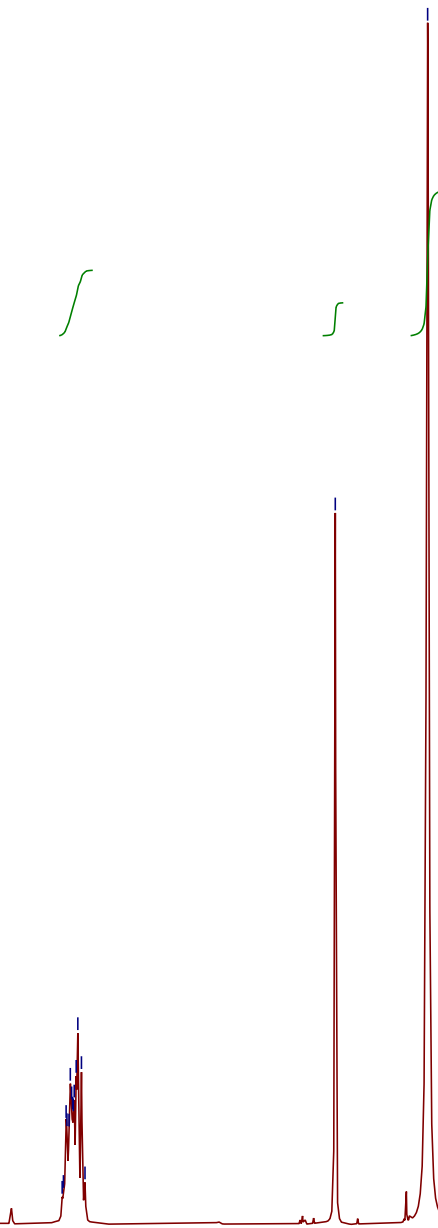

'T

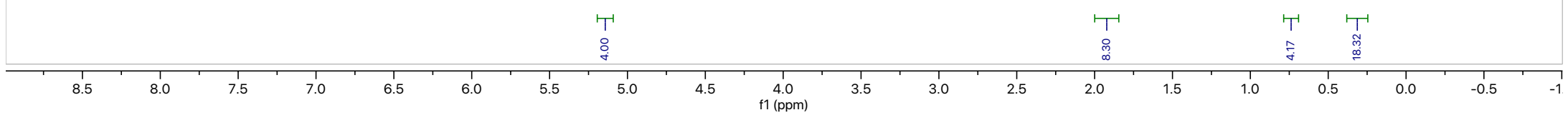


(cod)Pd(CH2TMS) 2 (P1)

${ }^{13} \mathrm{C}$ NMR (151 MHz, $\mathrm{C}_{6} \mathrm{D}_{6}$ )

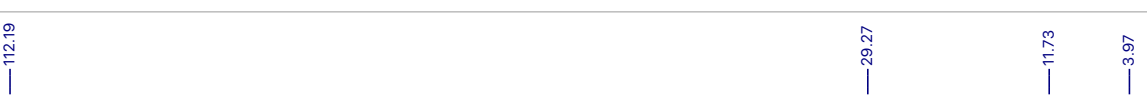

1. ${ }^{-\mathrm{TMS}}$
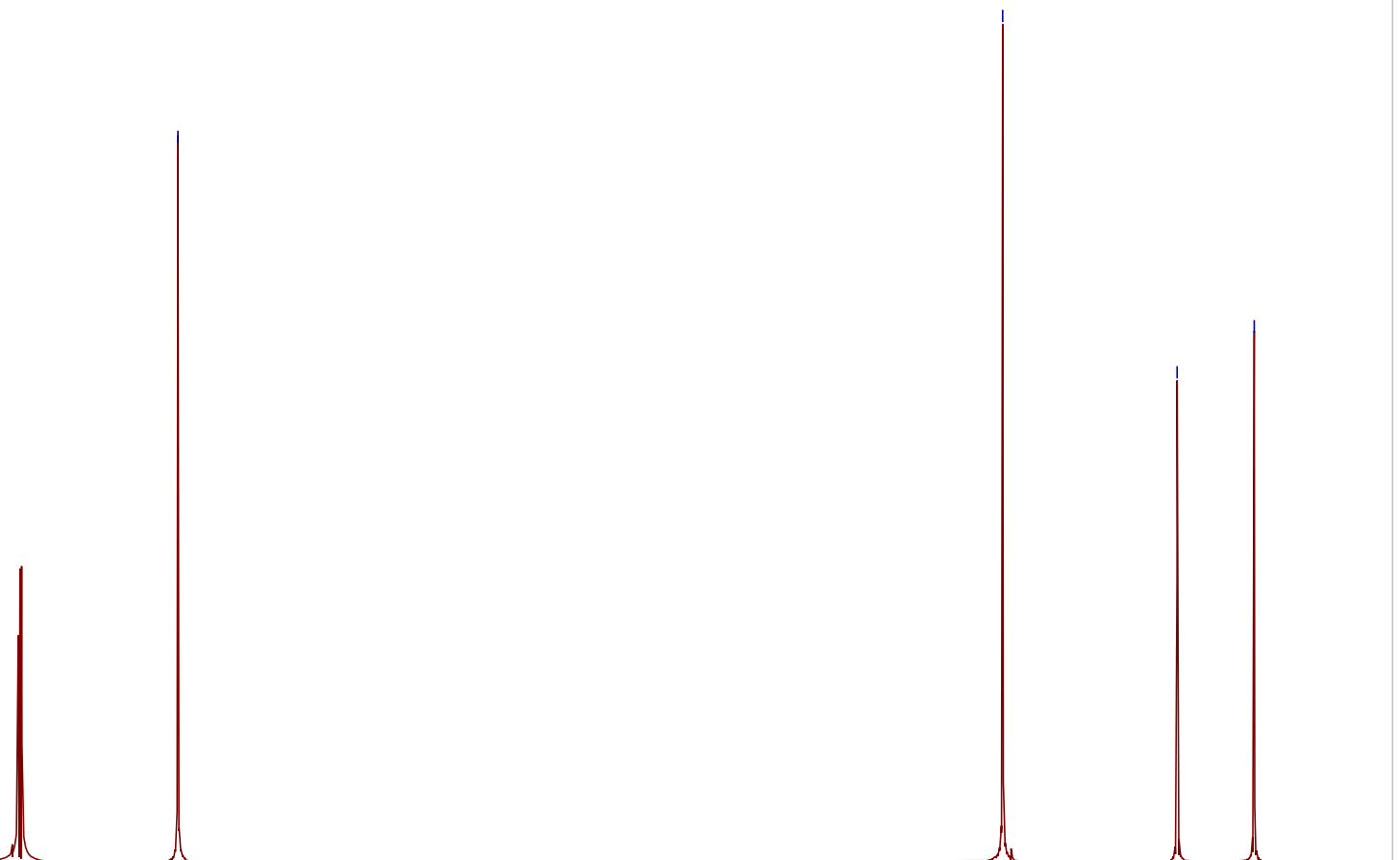

$190+180,170,160$

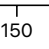

130

$120 \quad 110$

$100 \quad 90$

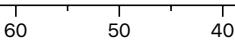

$\quad 30$ 1 
(cod)Pd(2,4,6-F 3 Ph) 2 (P2)

${ }^{1} \mathrm{H}$ NMR $\left(600 \mathrm{MHz}, \mathrm{CDCl}_{3}\right)$
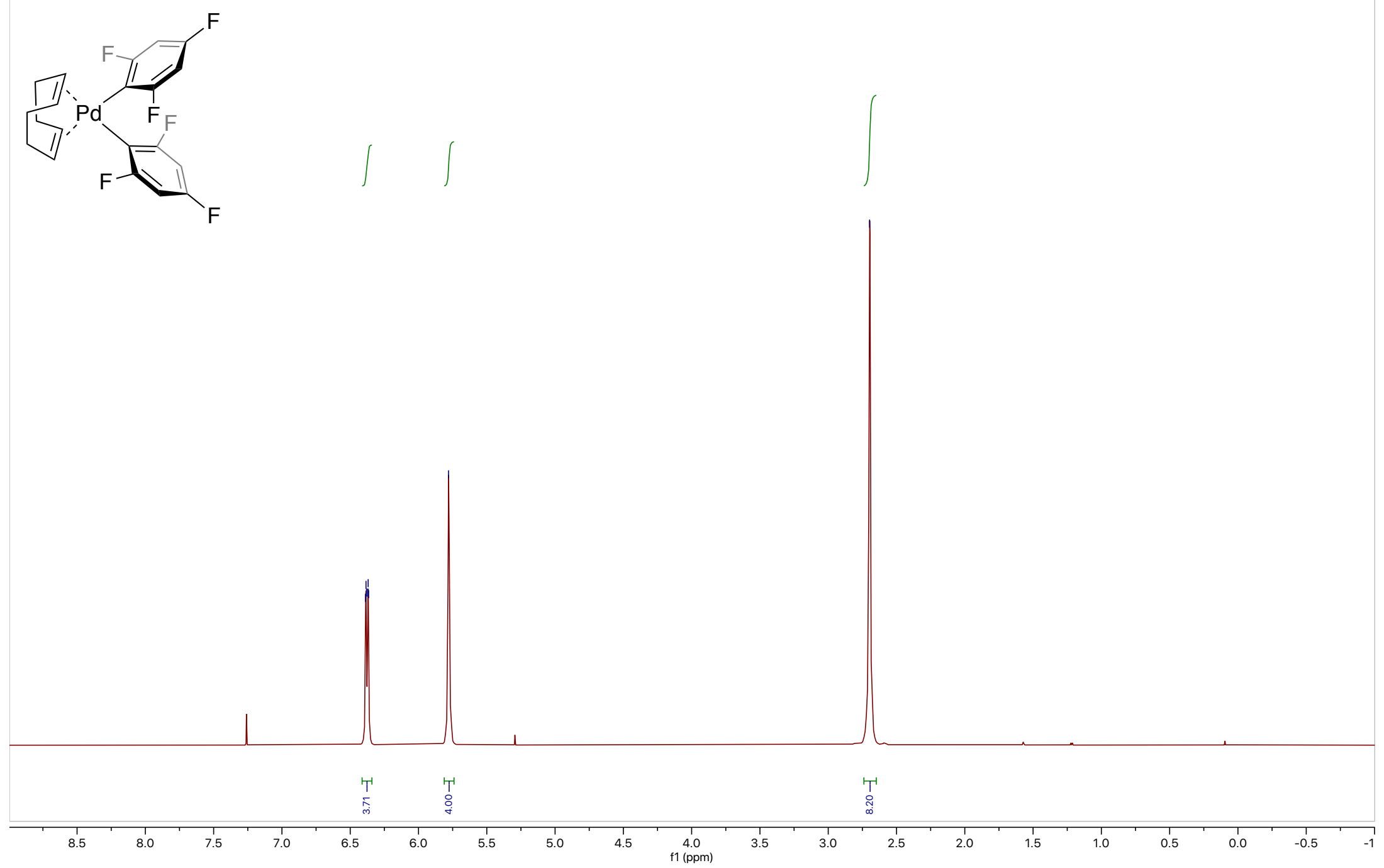


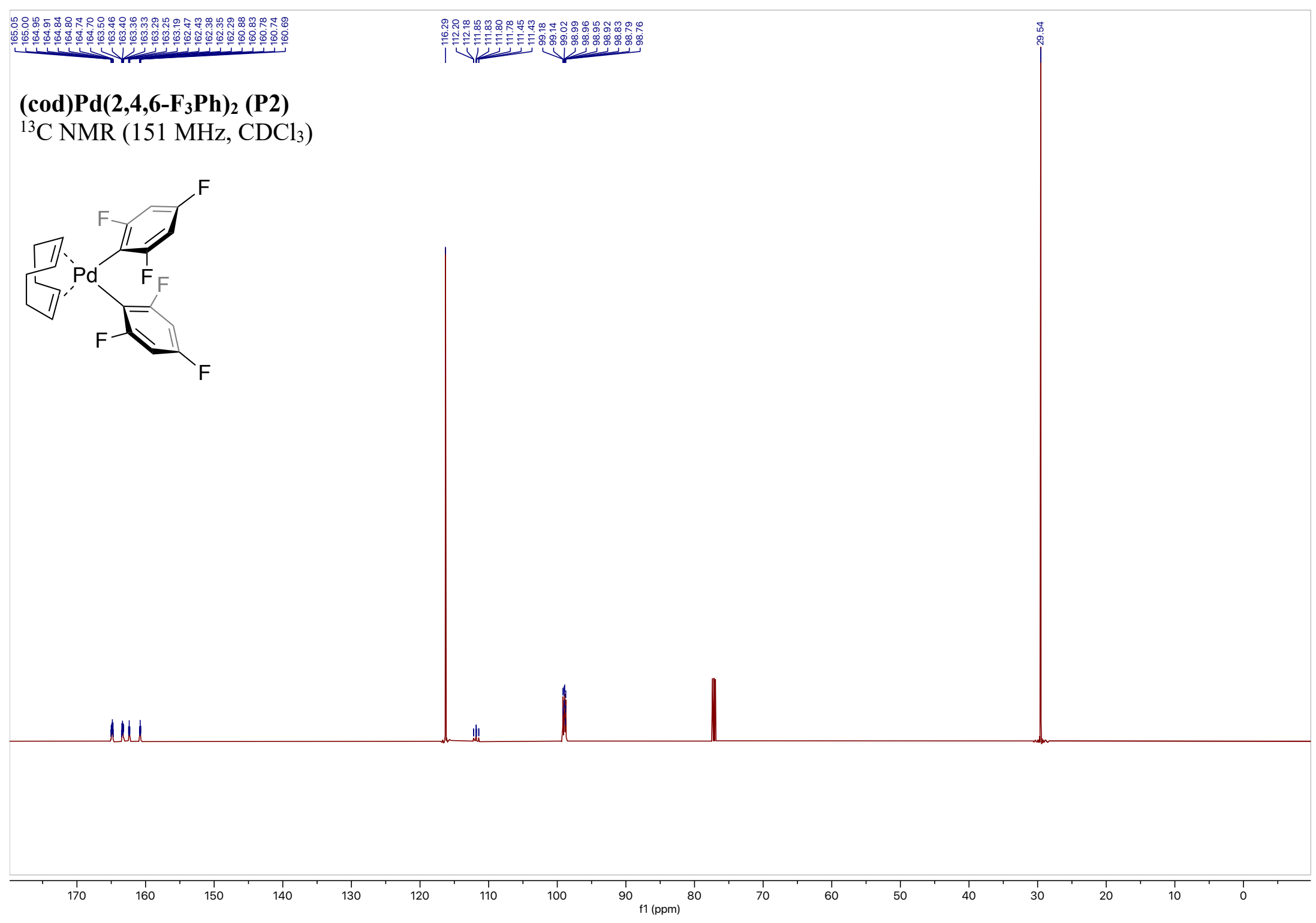


(cod)Pd(2,4,6-F 3 Ph) 2 (P2)

${ }^{19} \mathrm{~F}$ NMR (377 MHz, $\mathrm{CDCl}_{3}$ )

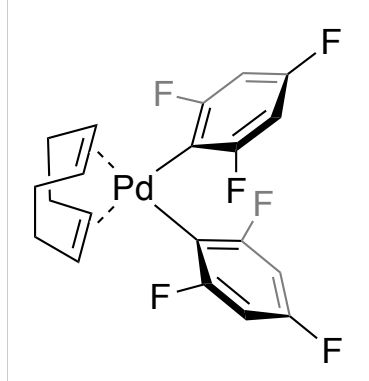

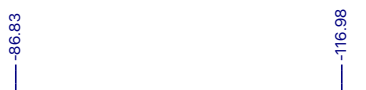

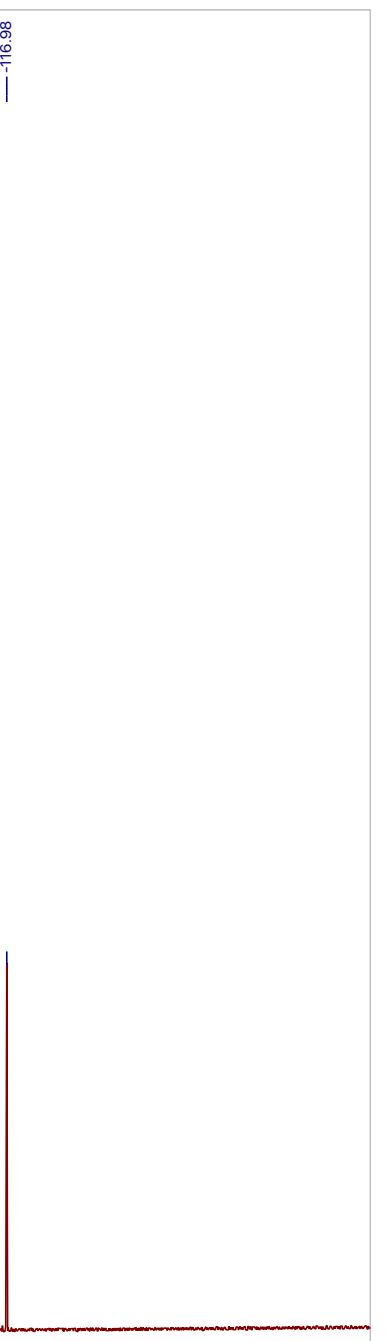

0

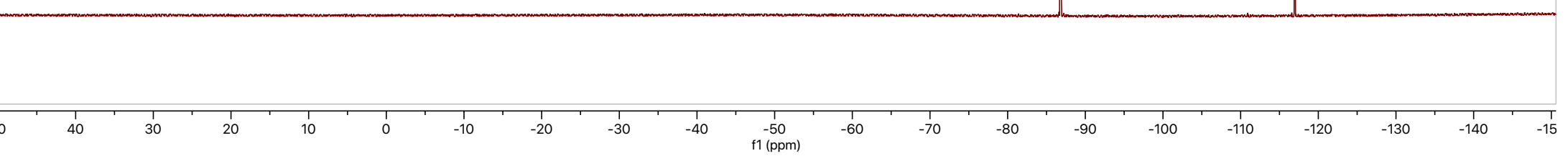

S57 


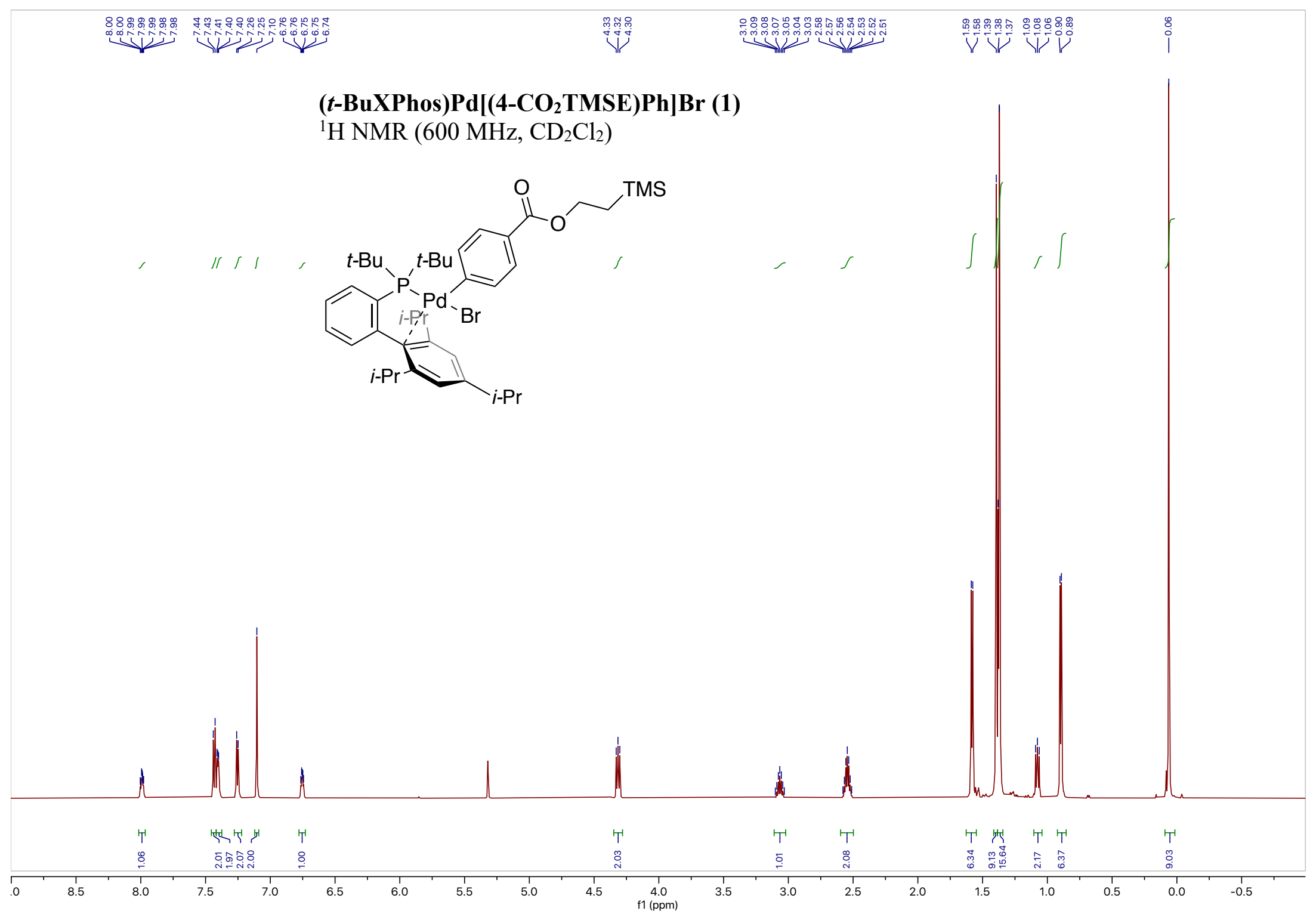









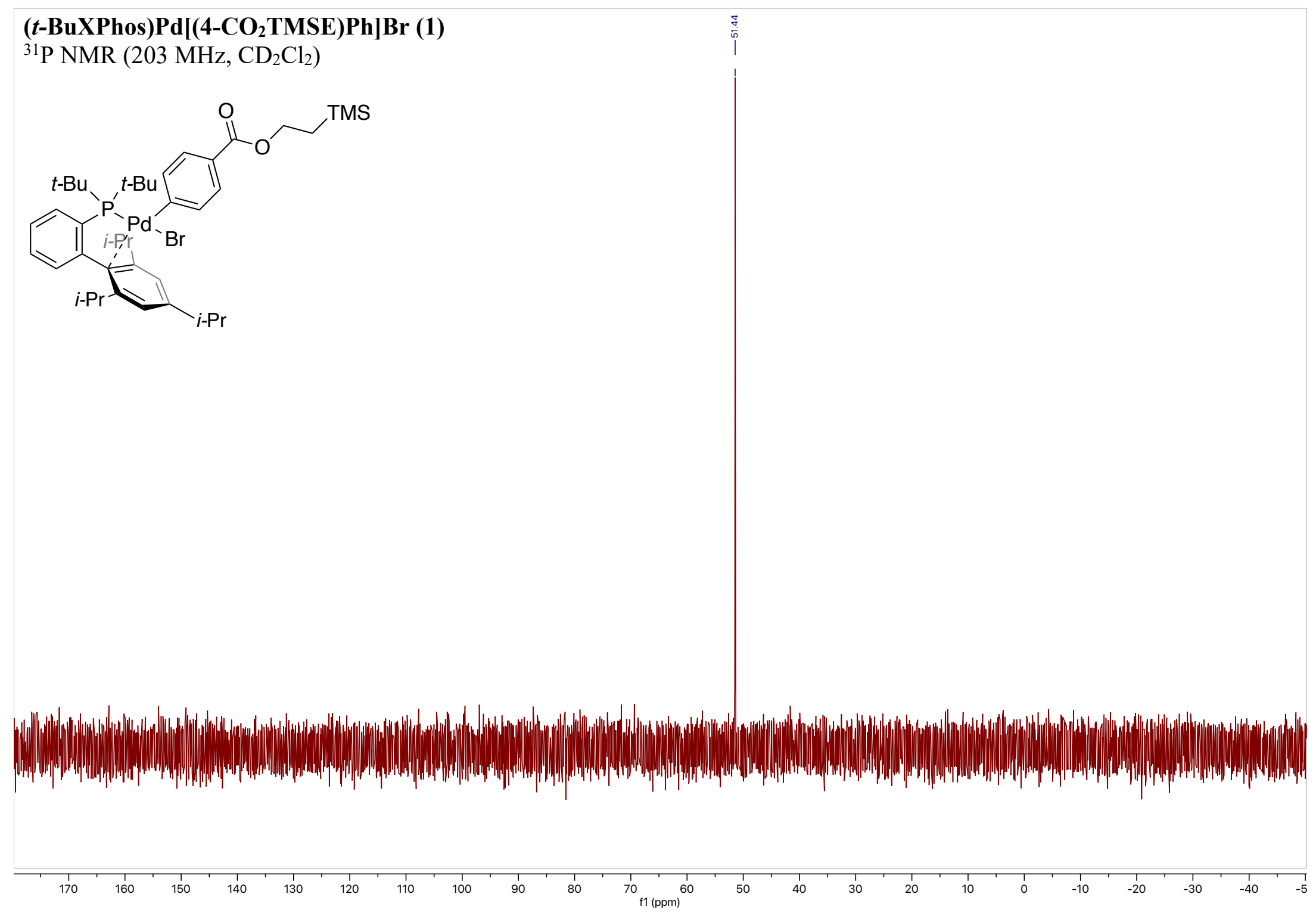




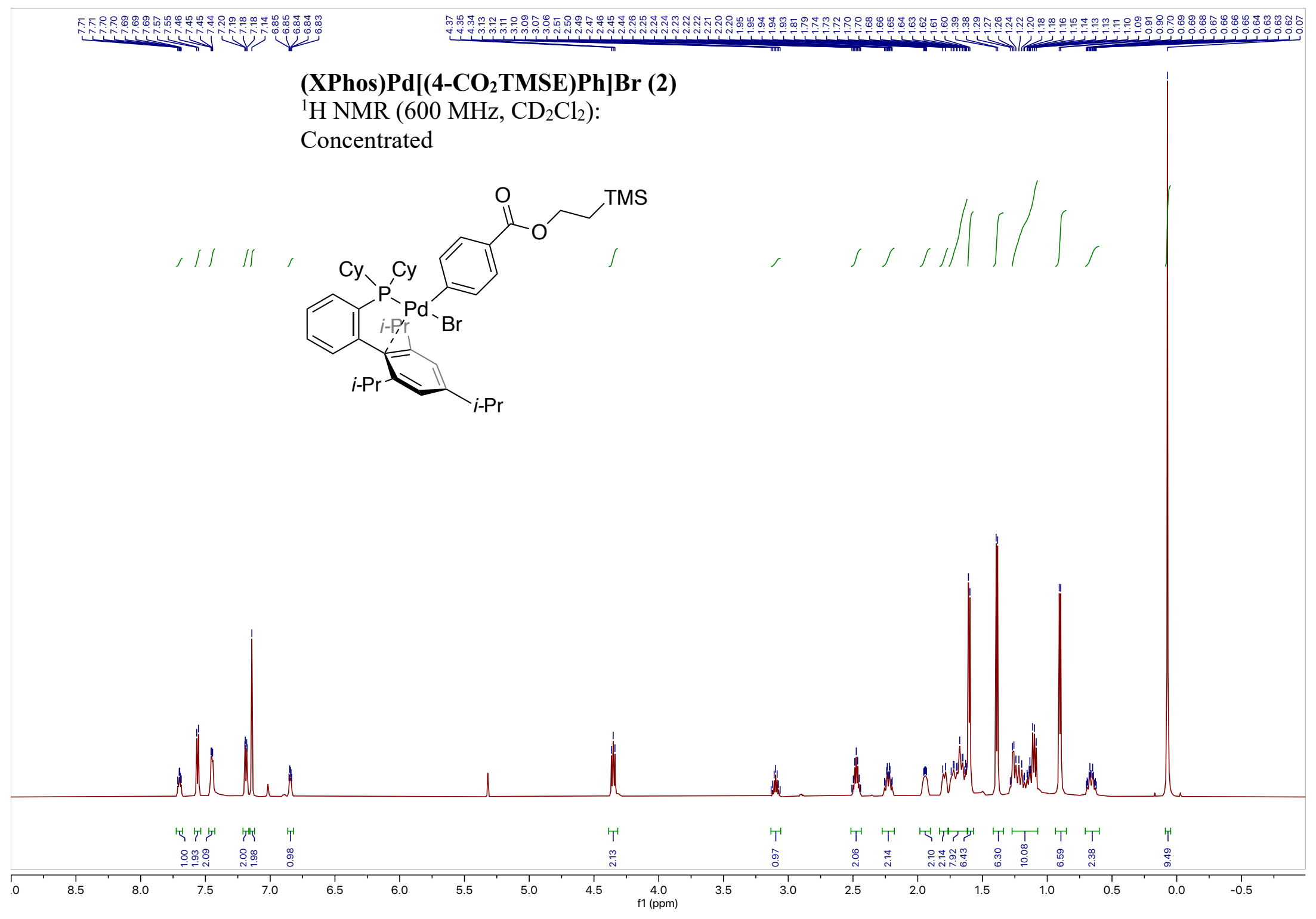




\section{(XPhos)Pd[(4-CO2TMSE)Ph]Br (2)}

${ }^{1} \mathrm{H}$ NMR (600 MHz, $\mathrm{CD}_{2} \mathrm{Cl}_{2}$ ):

Dilute
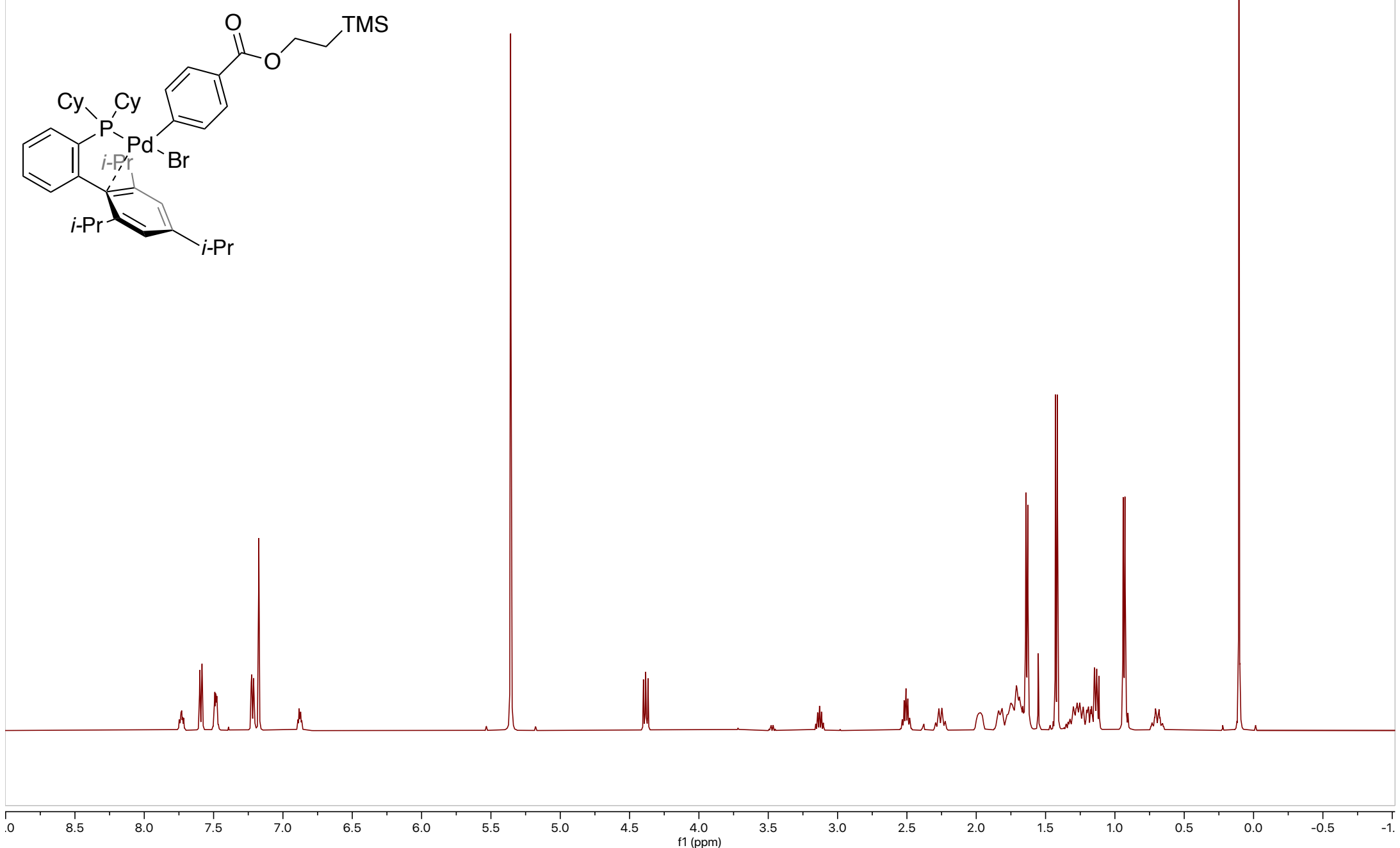


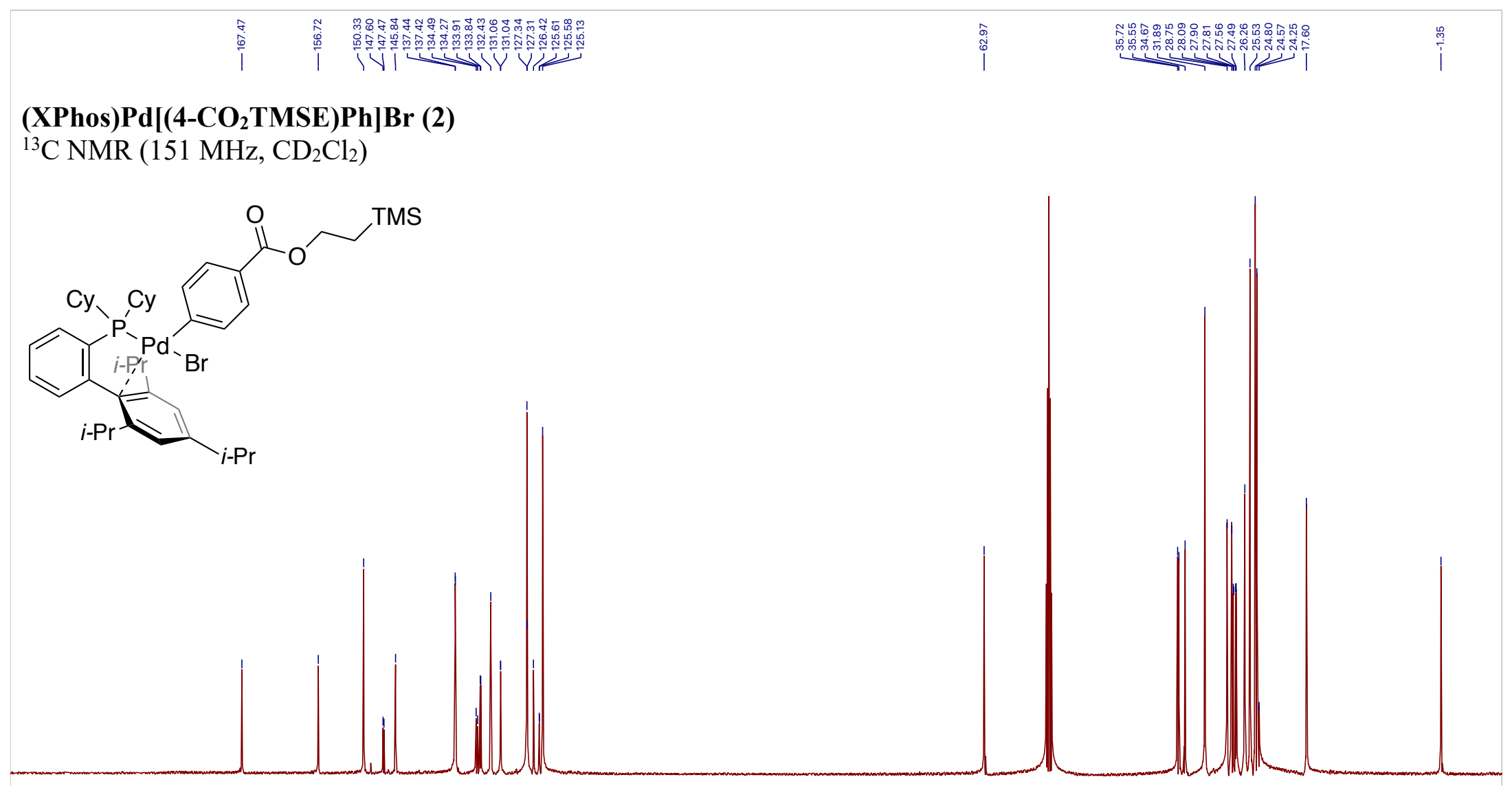


(XPhos)Pd[(4-CO2TMSE)Ph]Br (2)

${ }^{31} \mathrm{P}$ NMR (203 MHz, $\mathrm{CD}_{2} \mathrm{Cl}_{2}$ )
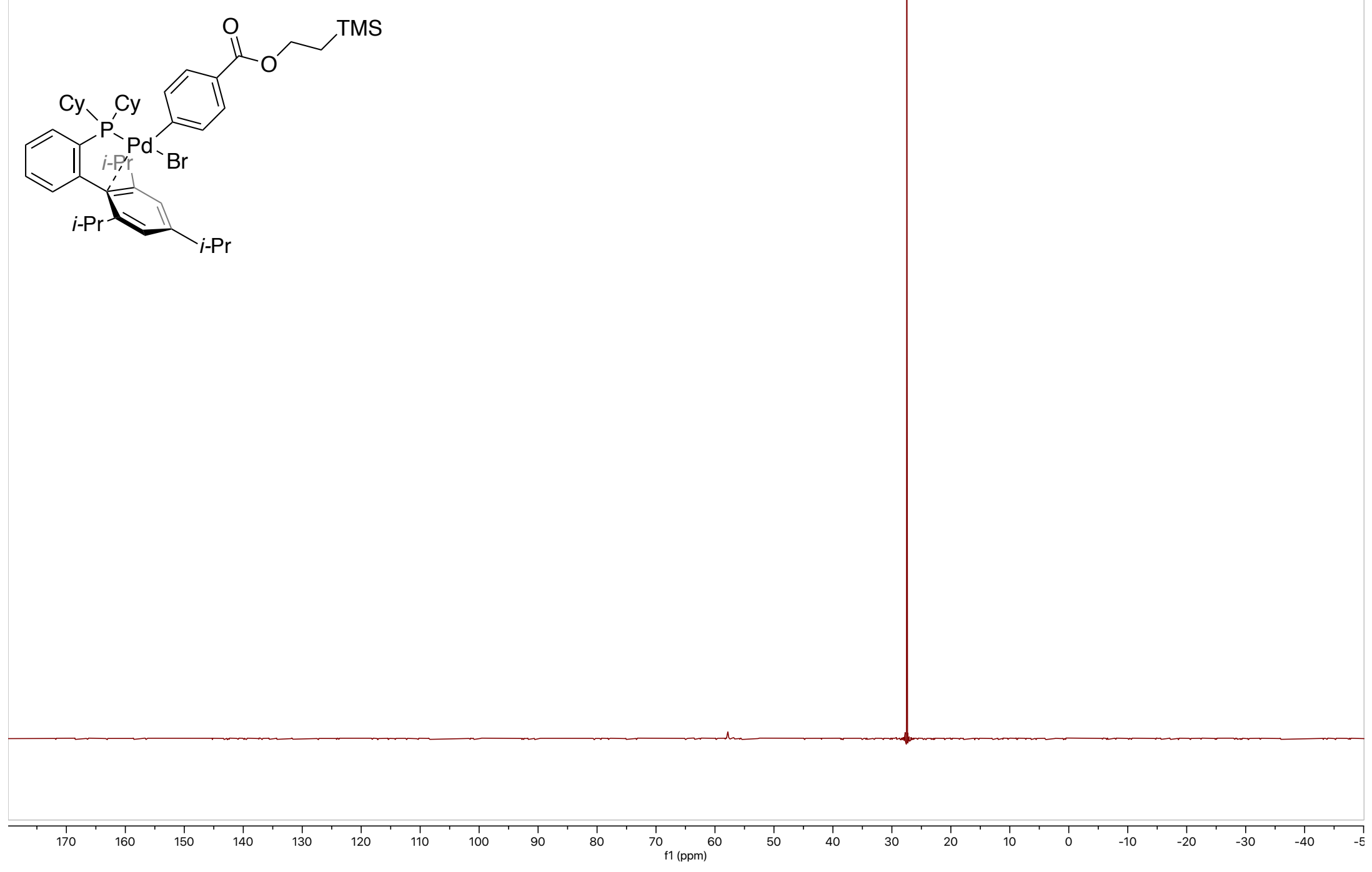







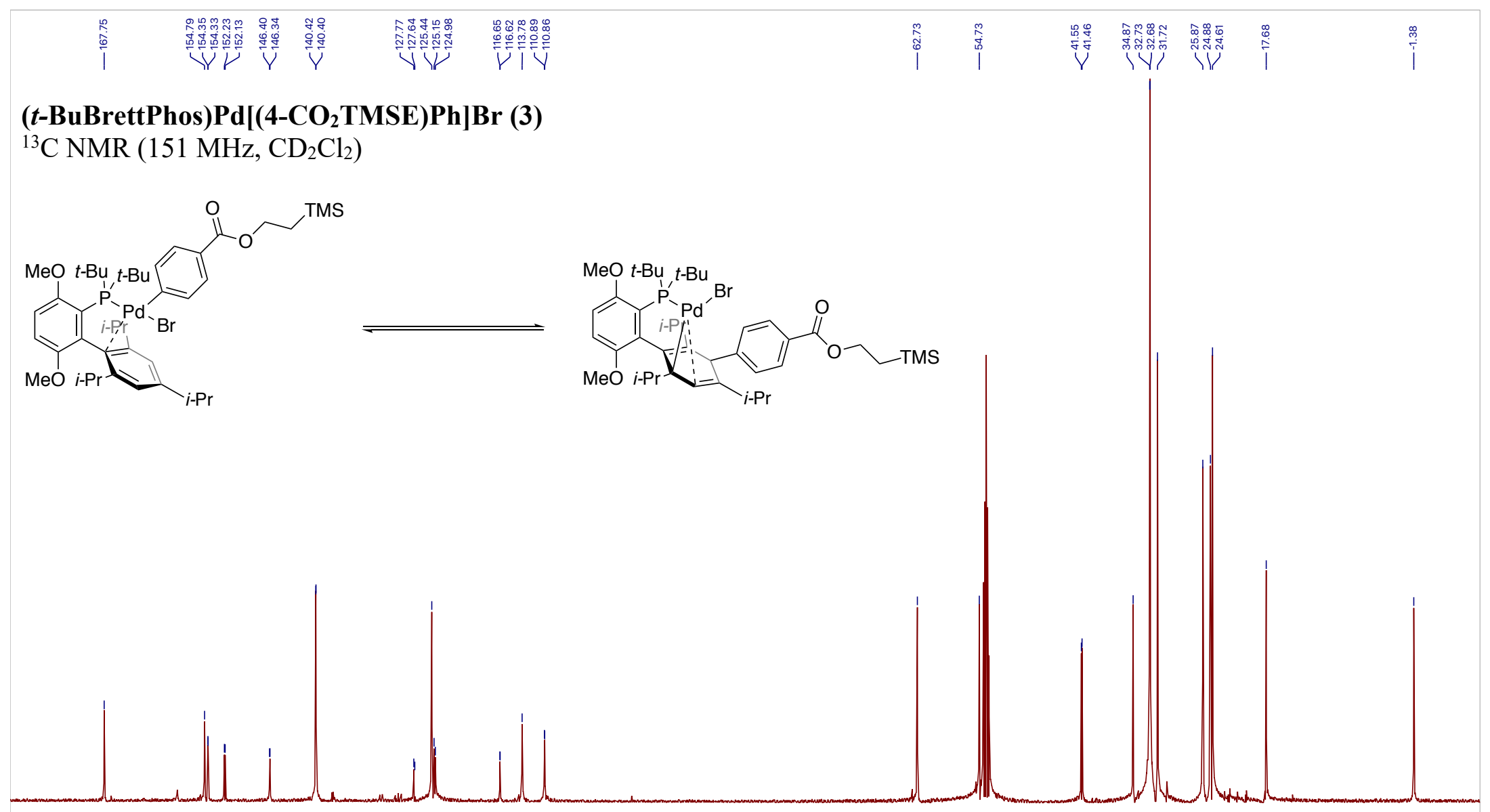




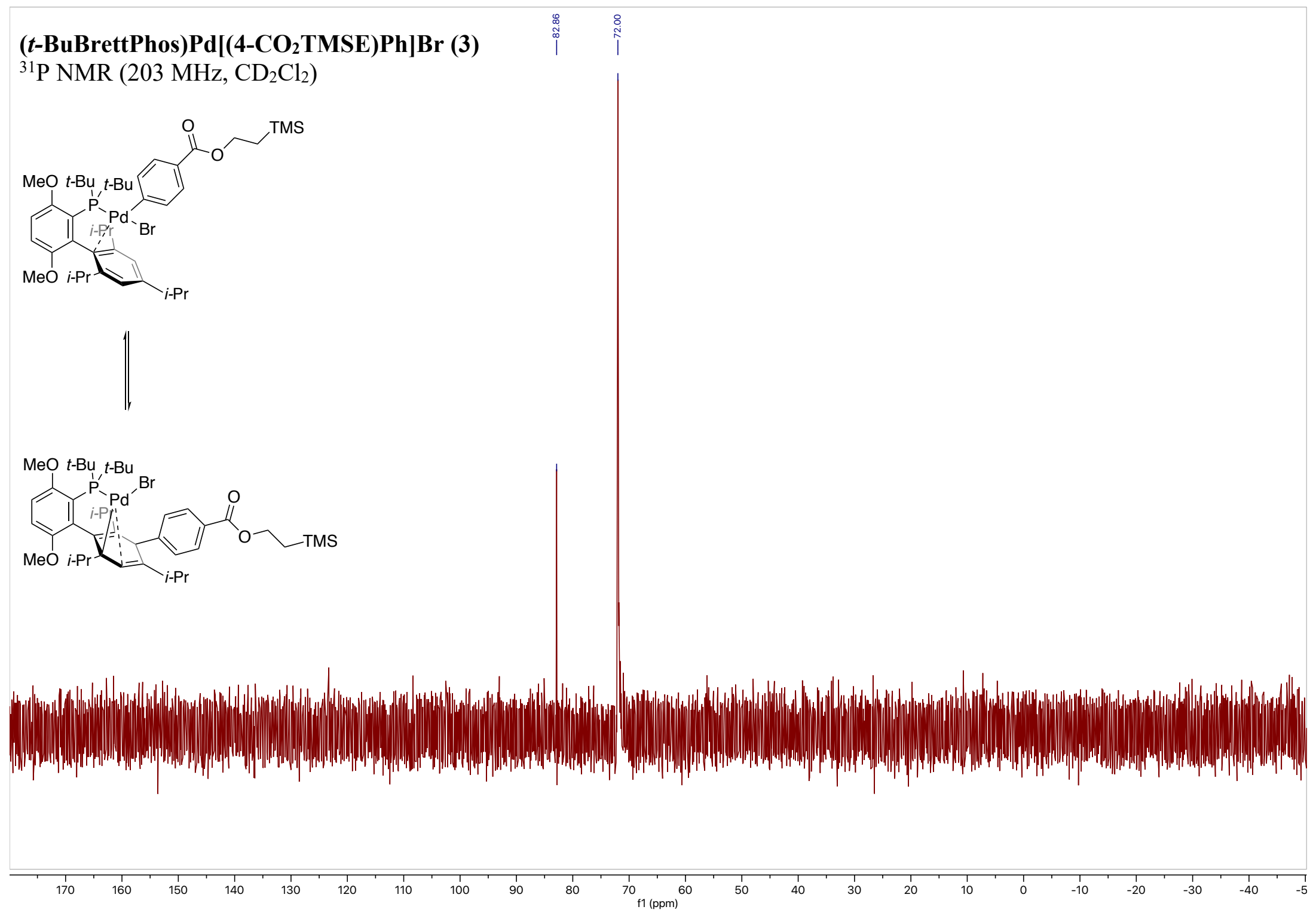




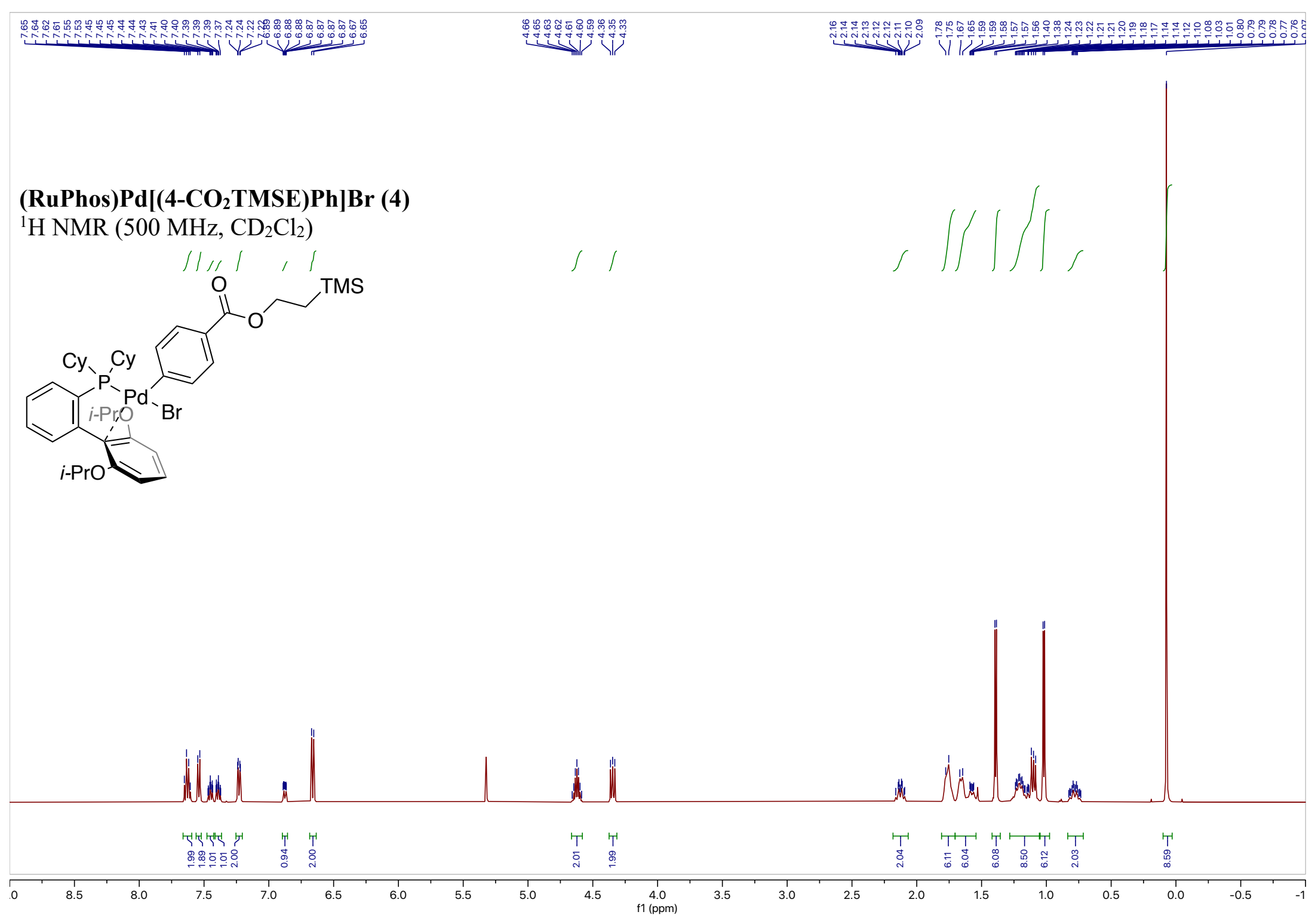




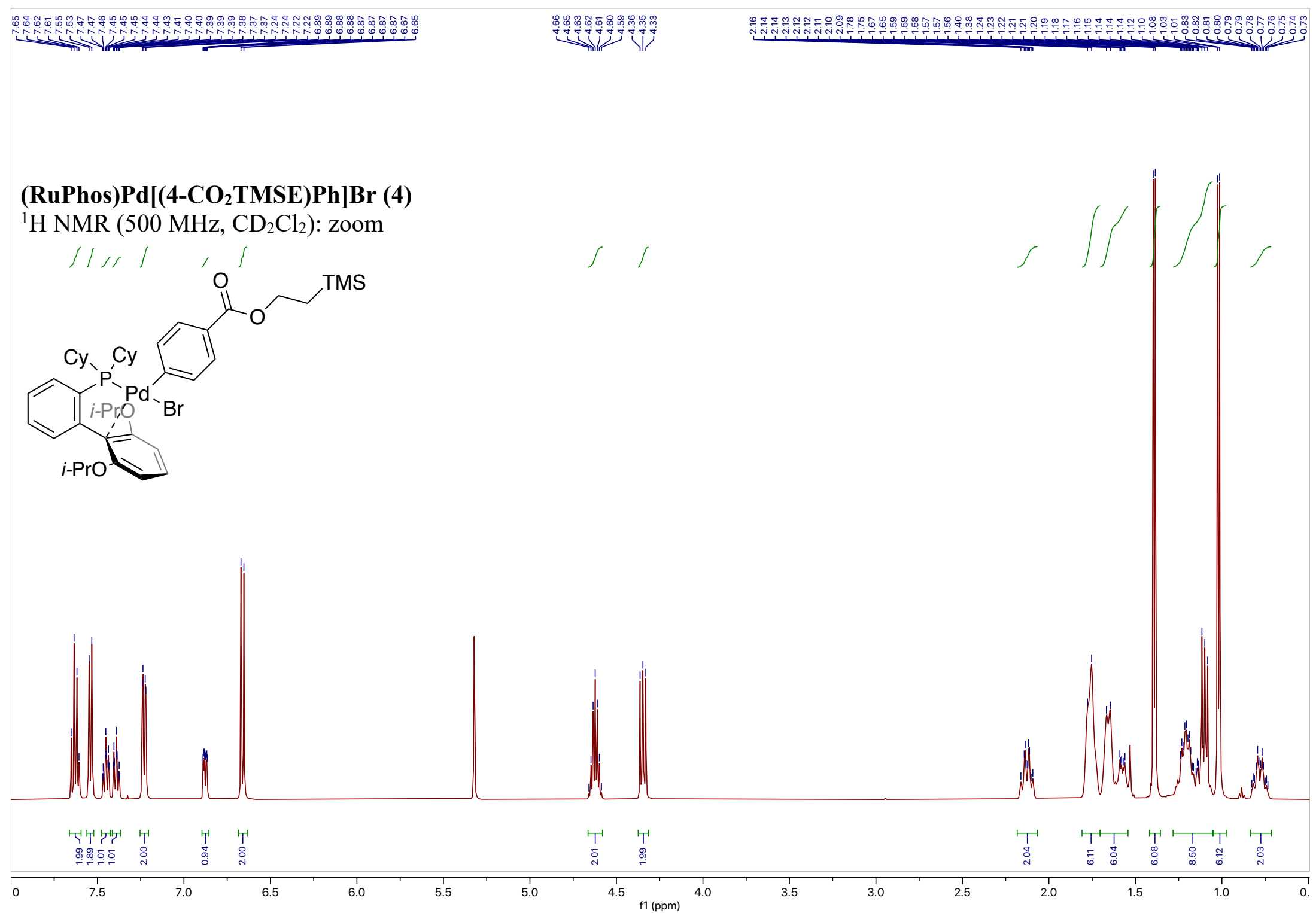




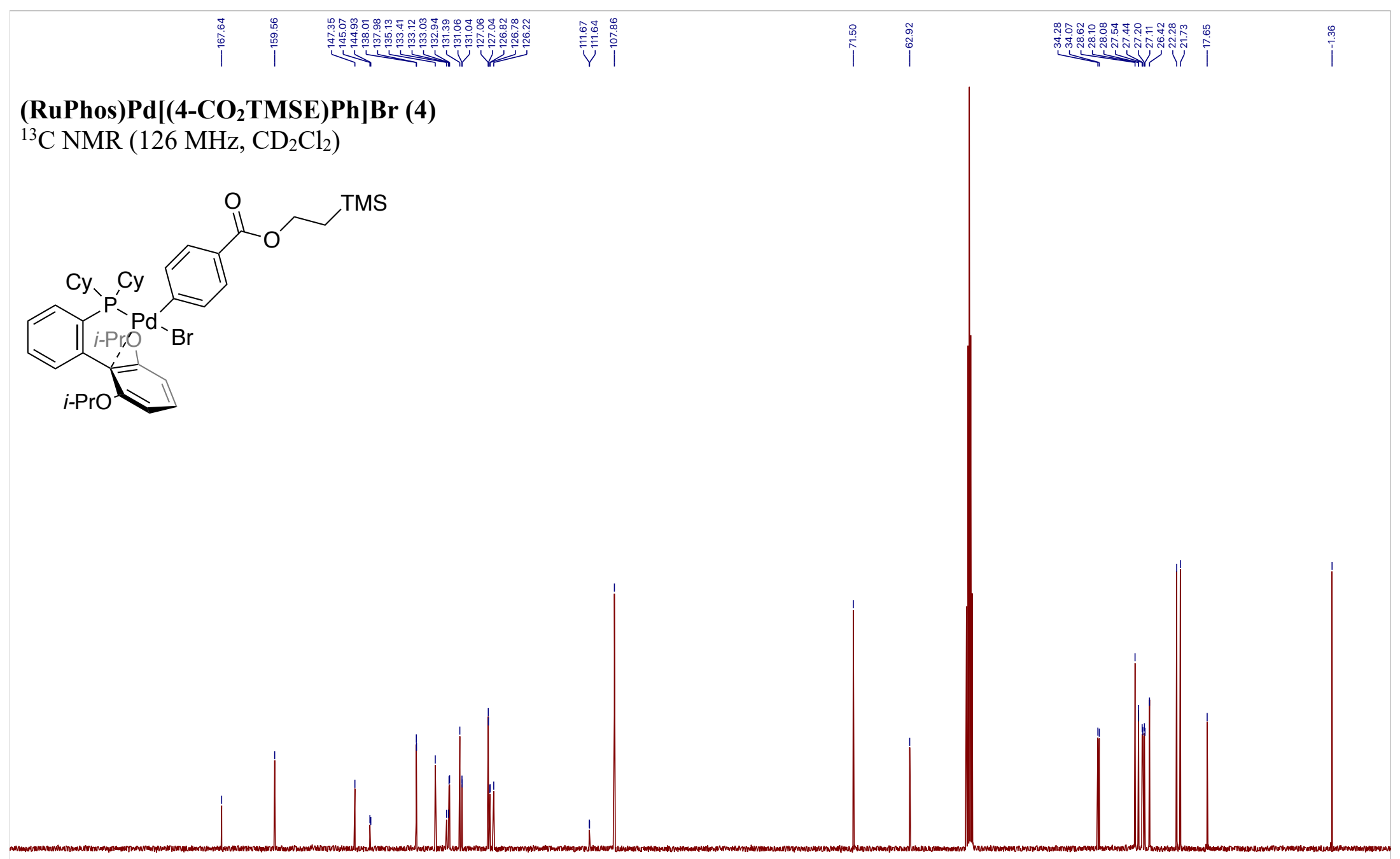


(RuPhos)Pd[(4-CO2 TMSE)Ph]Br (4)

${ }^{31} \mathrm{P}$ NMR (203 MHz, $\mathrm{CD}_{2} \mathrm{Cl}_{2}$ )

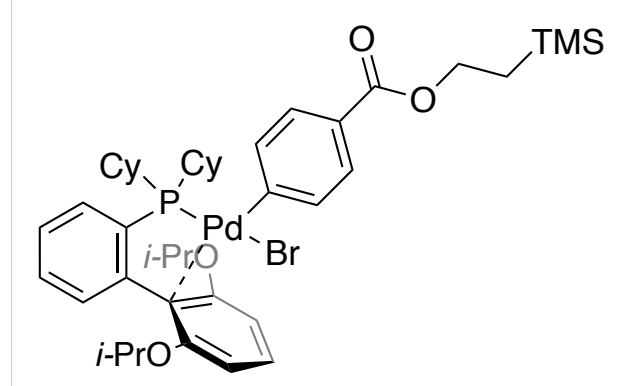









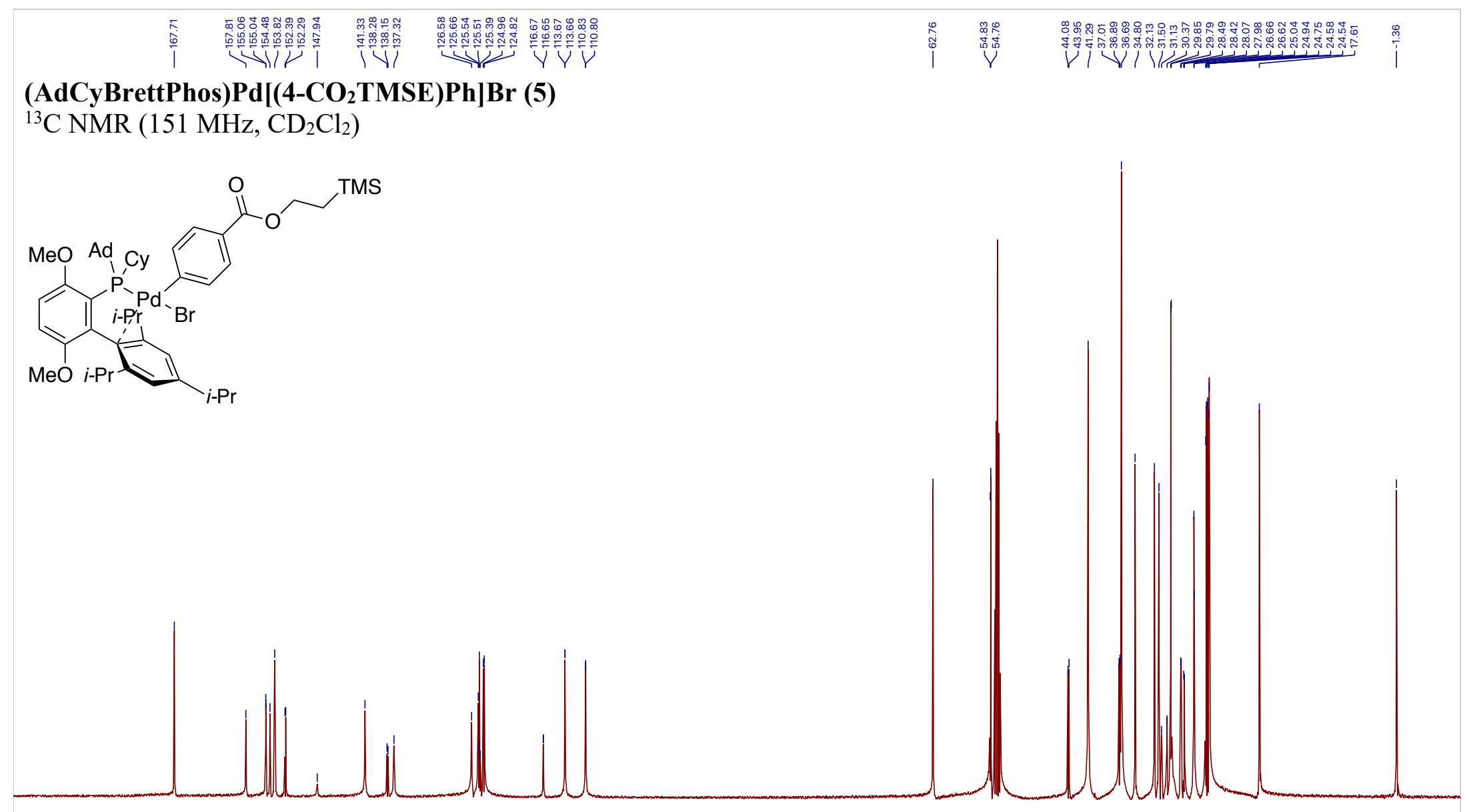


(AdCyBrettPhos)Pd[(4-CO2TMSE)Ph]Br (5)

${ }^{31} \mathrm{P}$ NMR $\left(162 \mathrm{MHz}, \mathrm{CD}_{2} \mathrm{Cl}_{2}\right)$

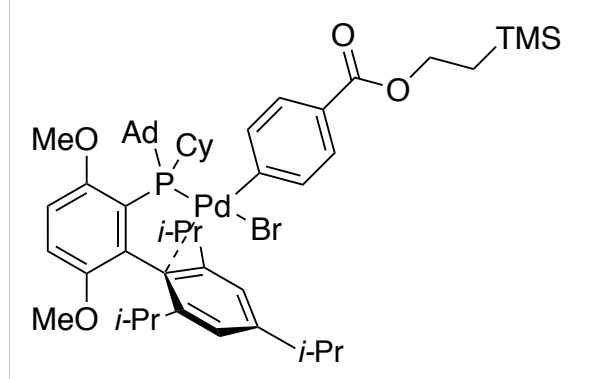

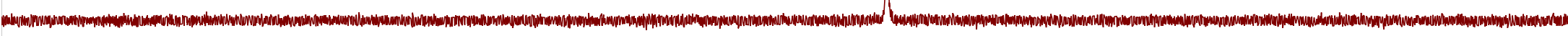




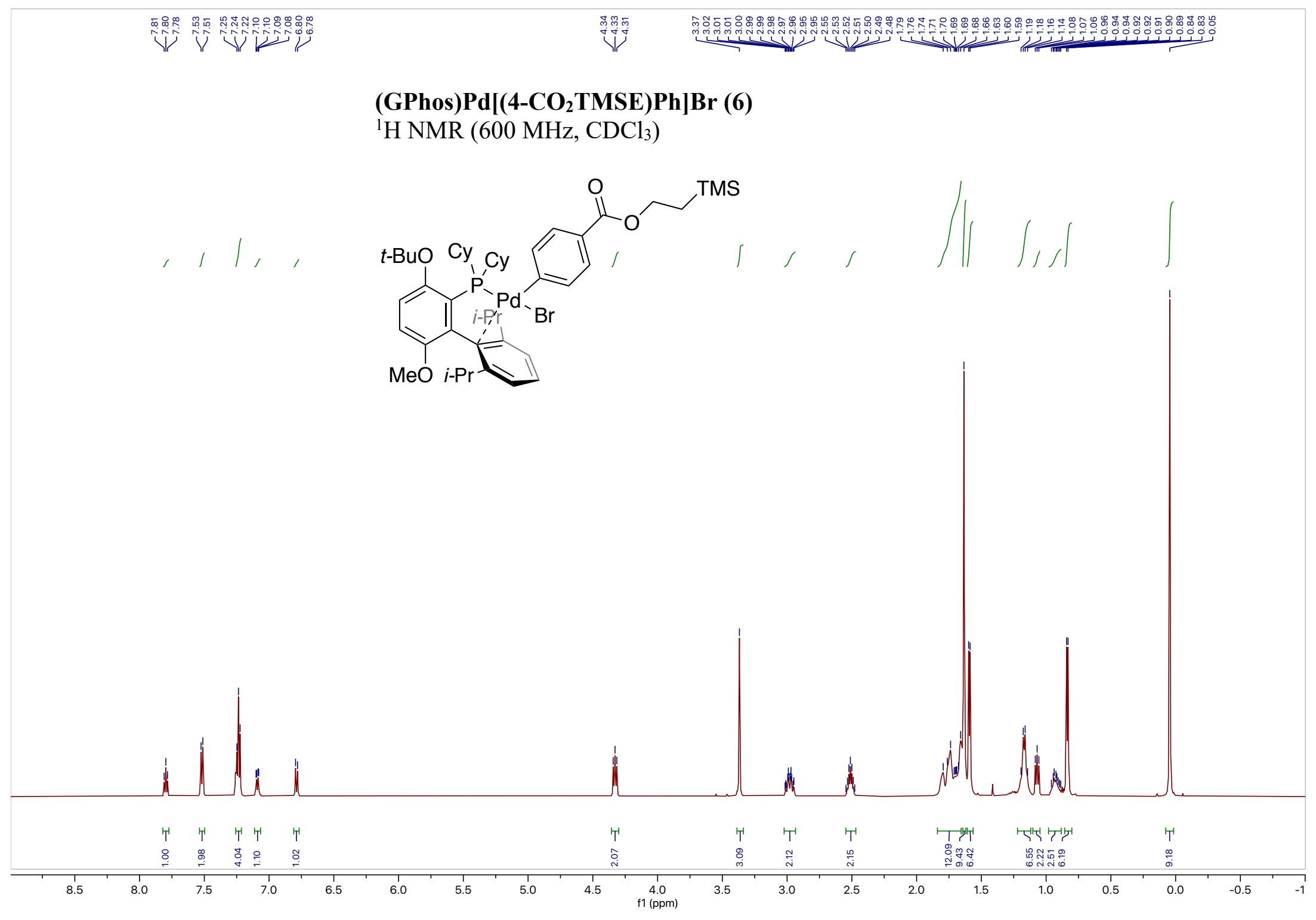




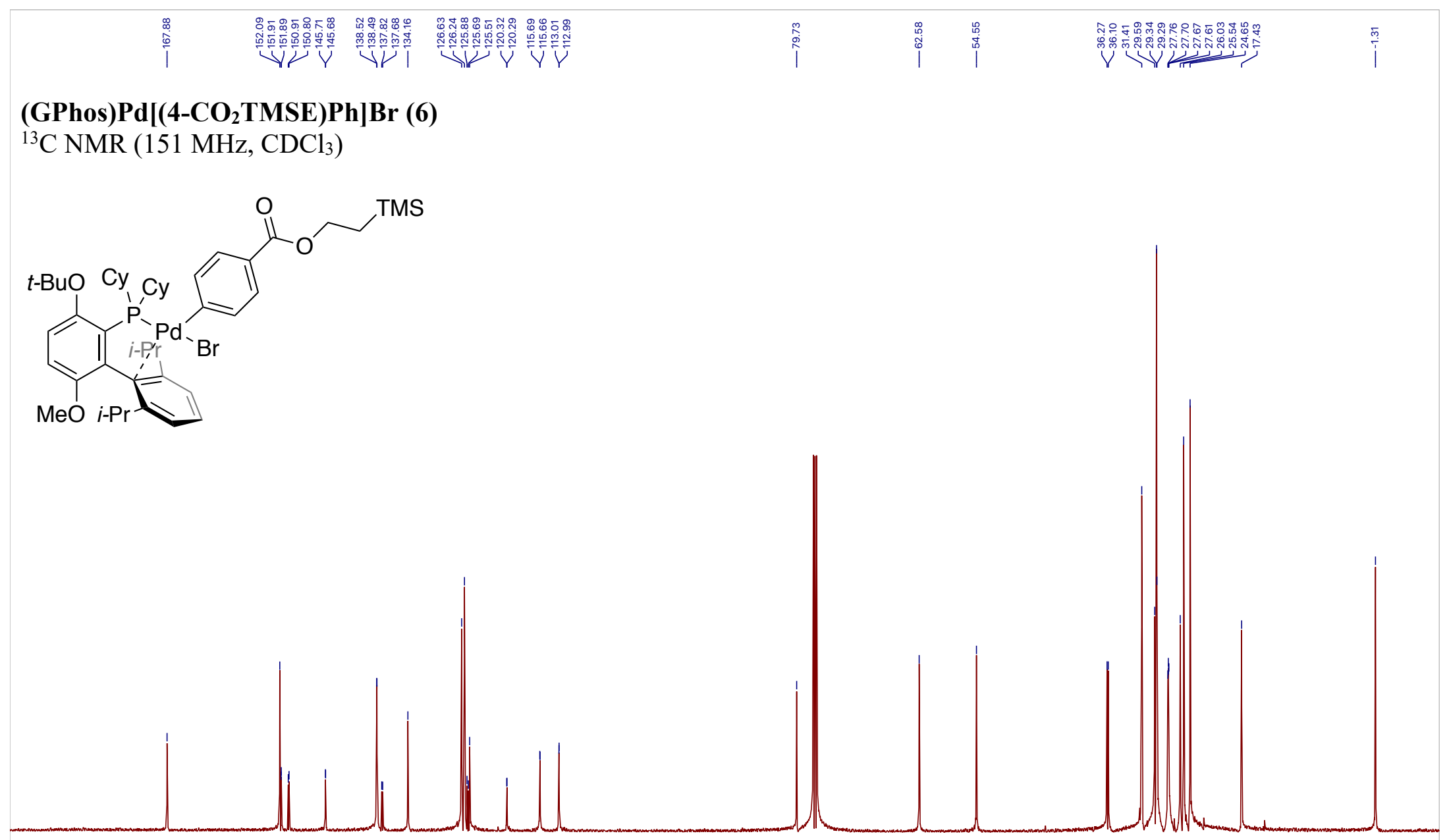


(GPhos)Pd[(4-CO2TMSE)Ph]Br (6)

${ }^{31} \mathrm{P}$ NMR (203 MHz, $\mathrm{CDCl}_{3}$ )



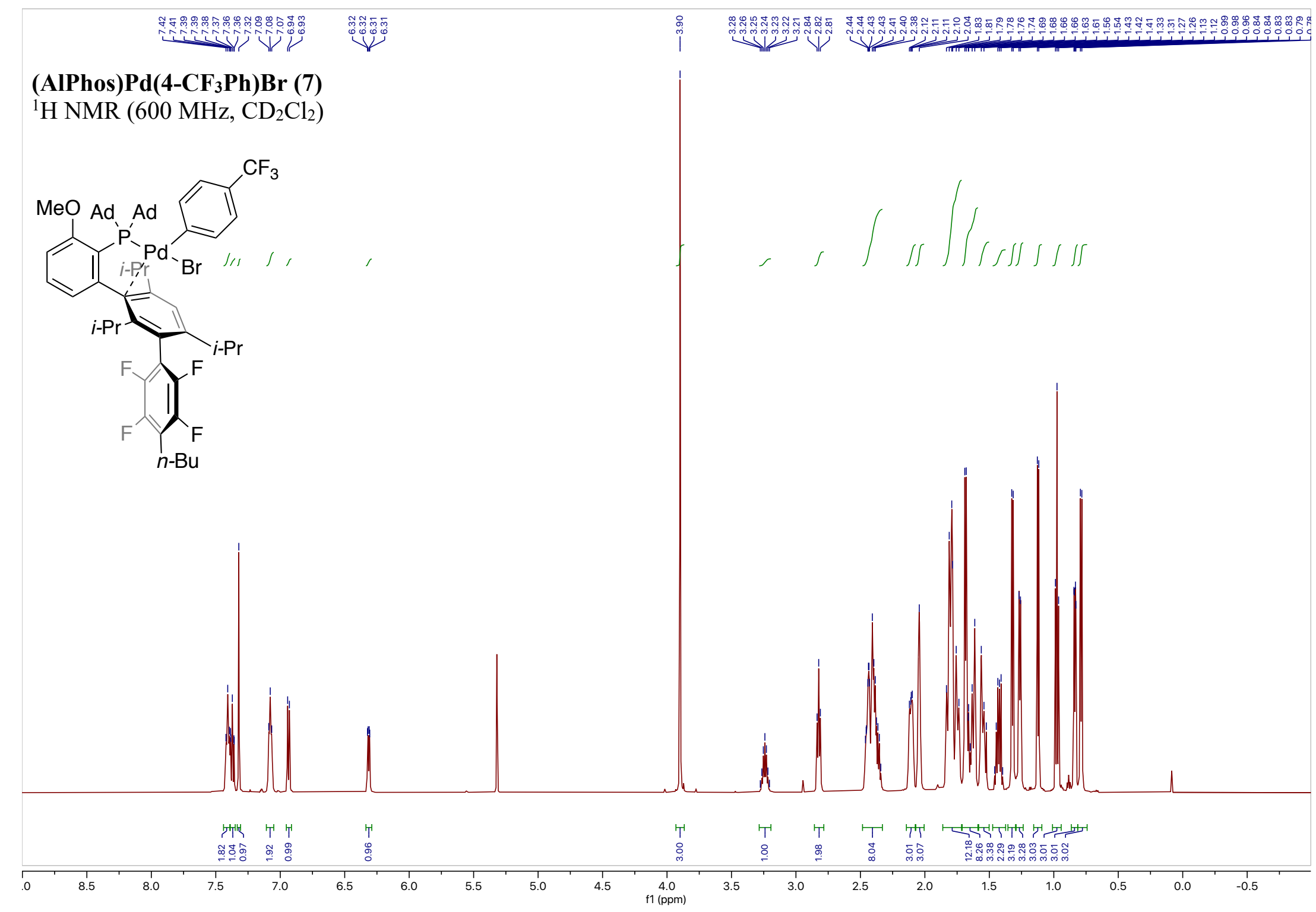




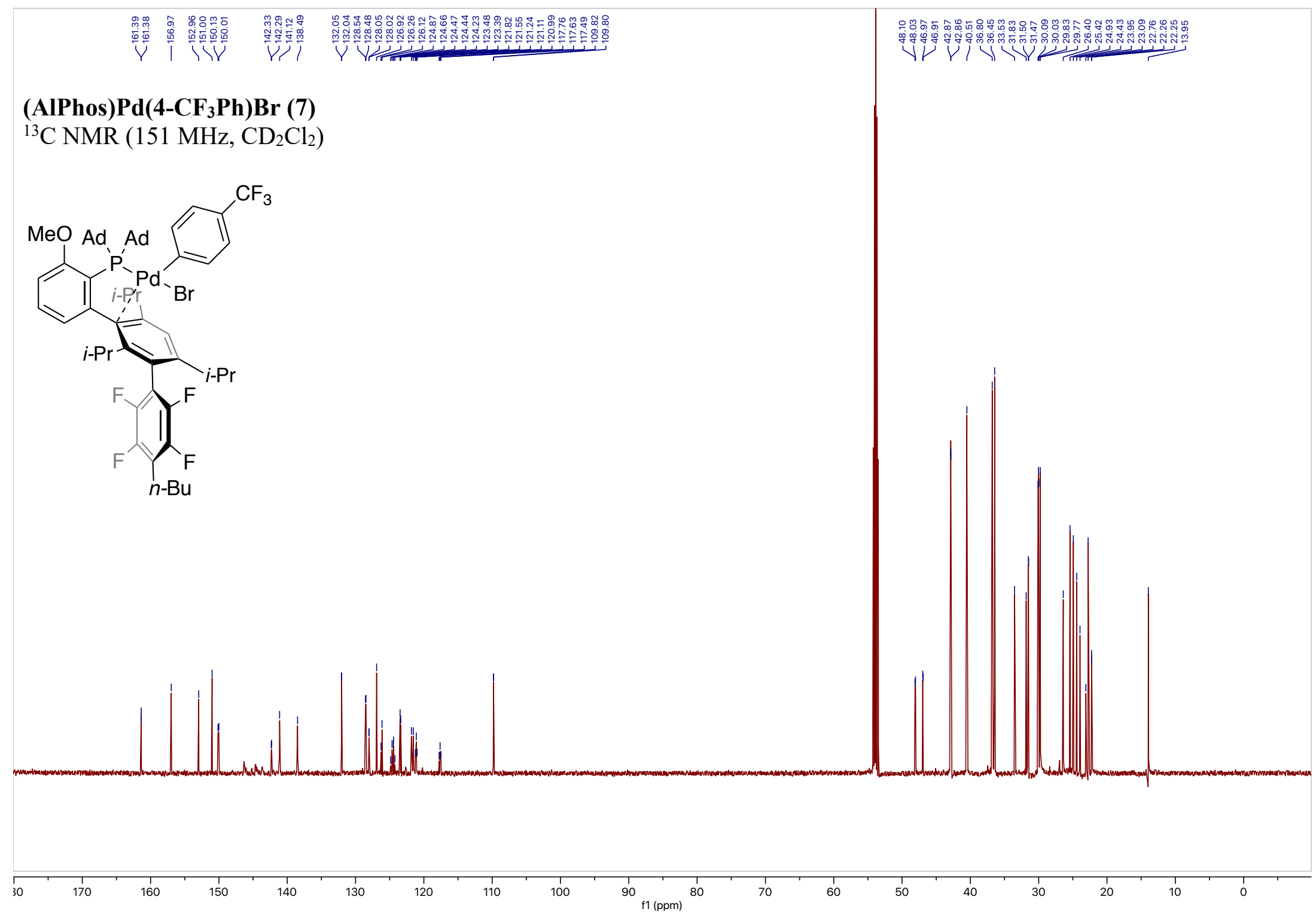









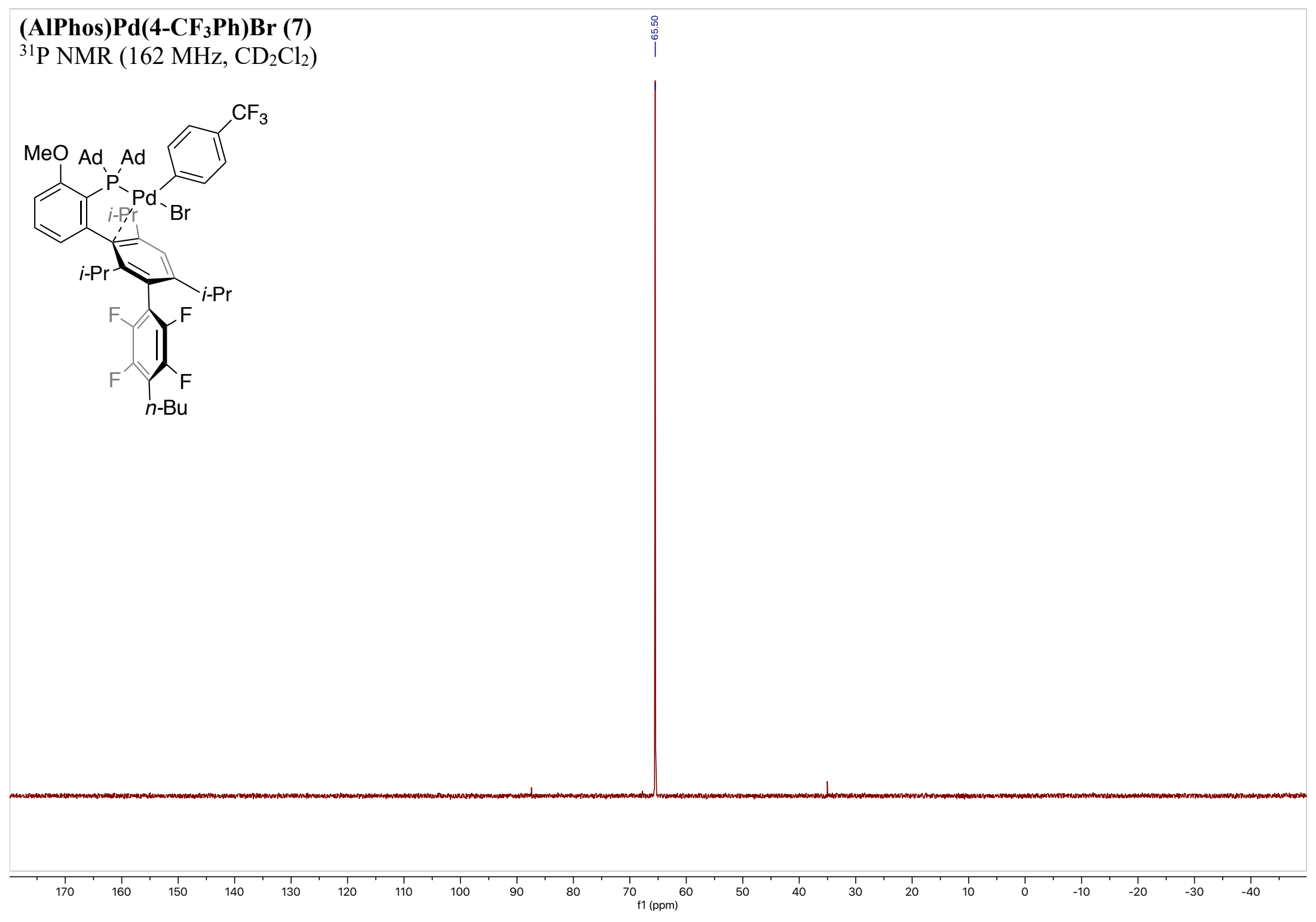




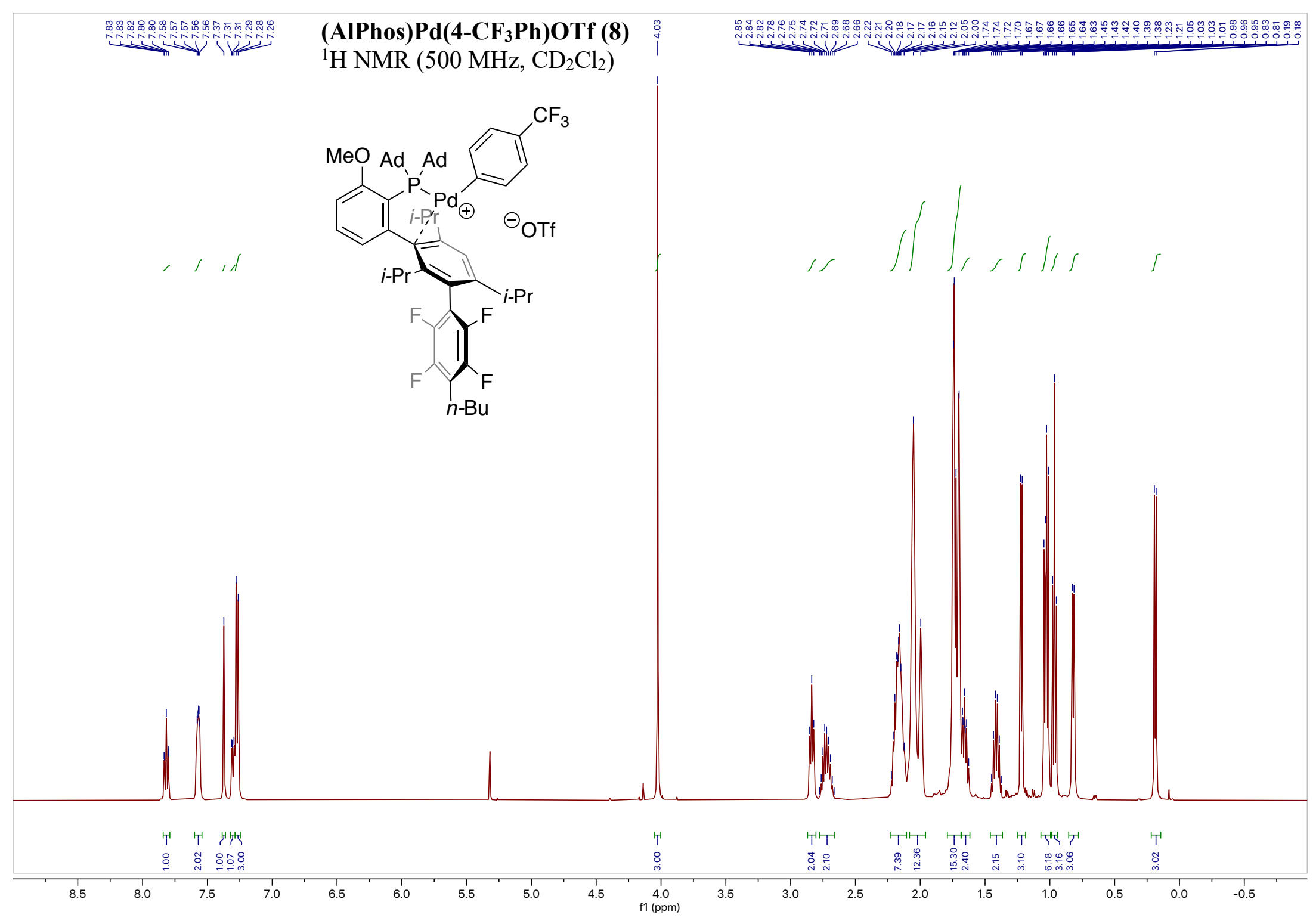




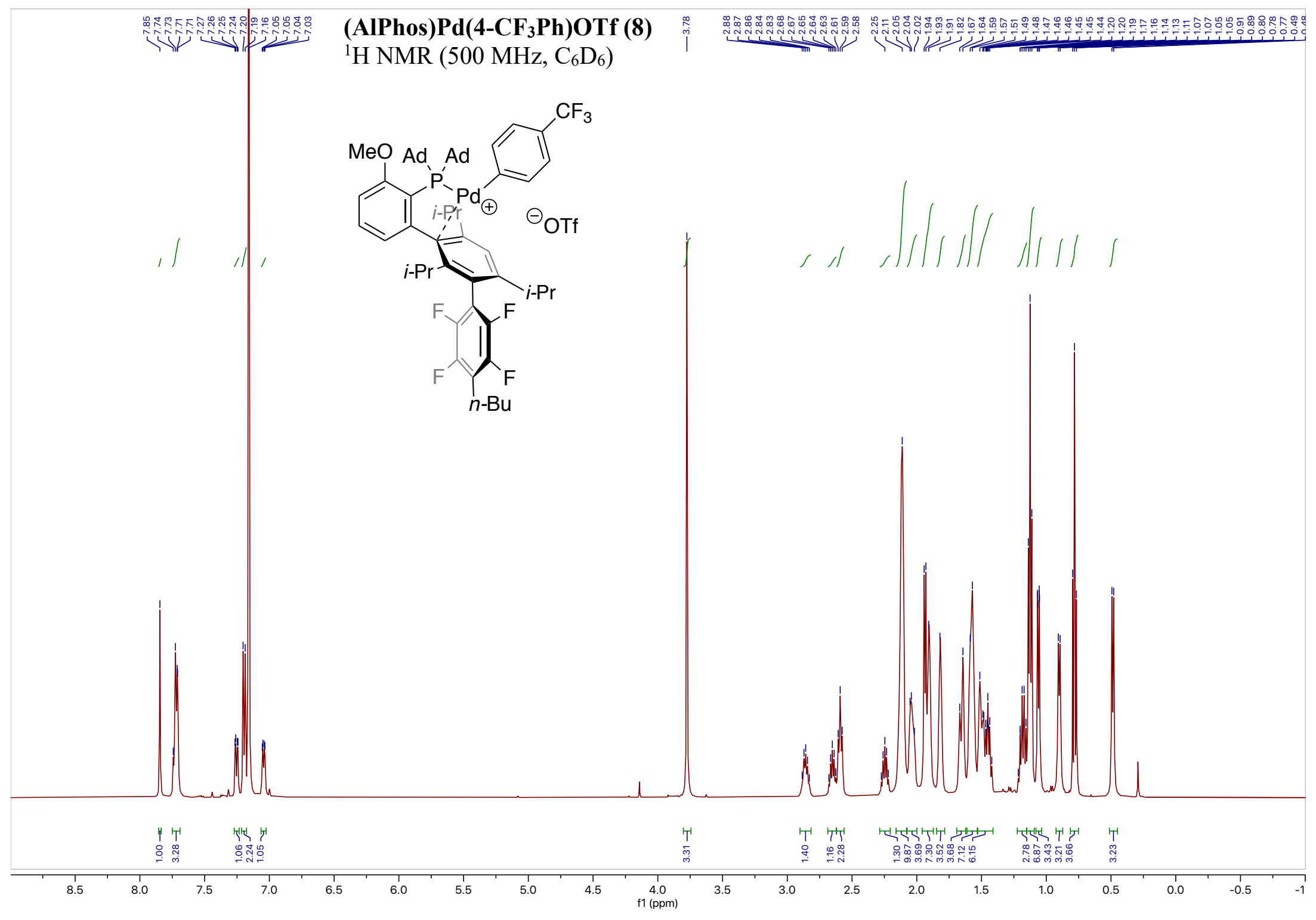




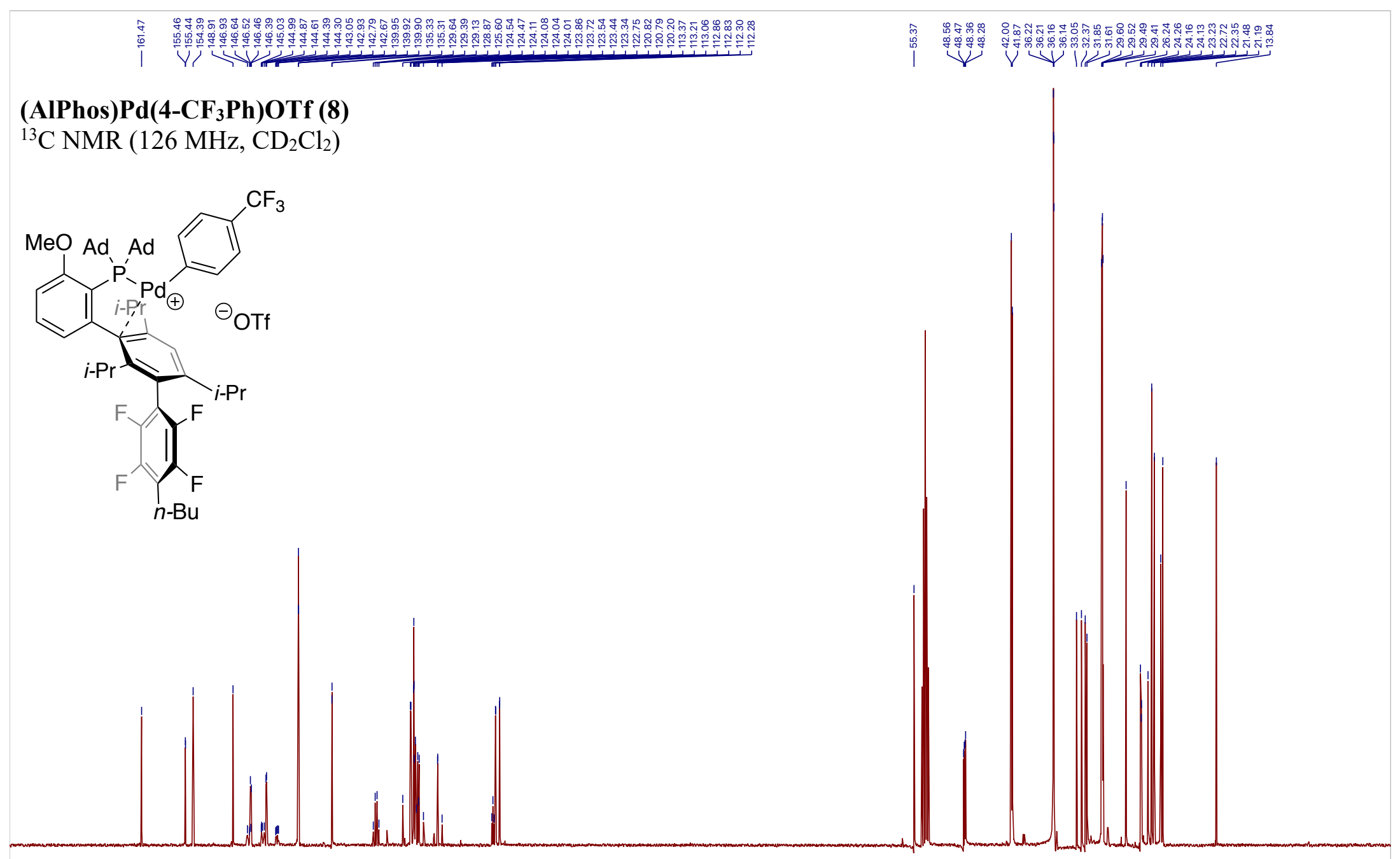




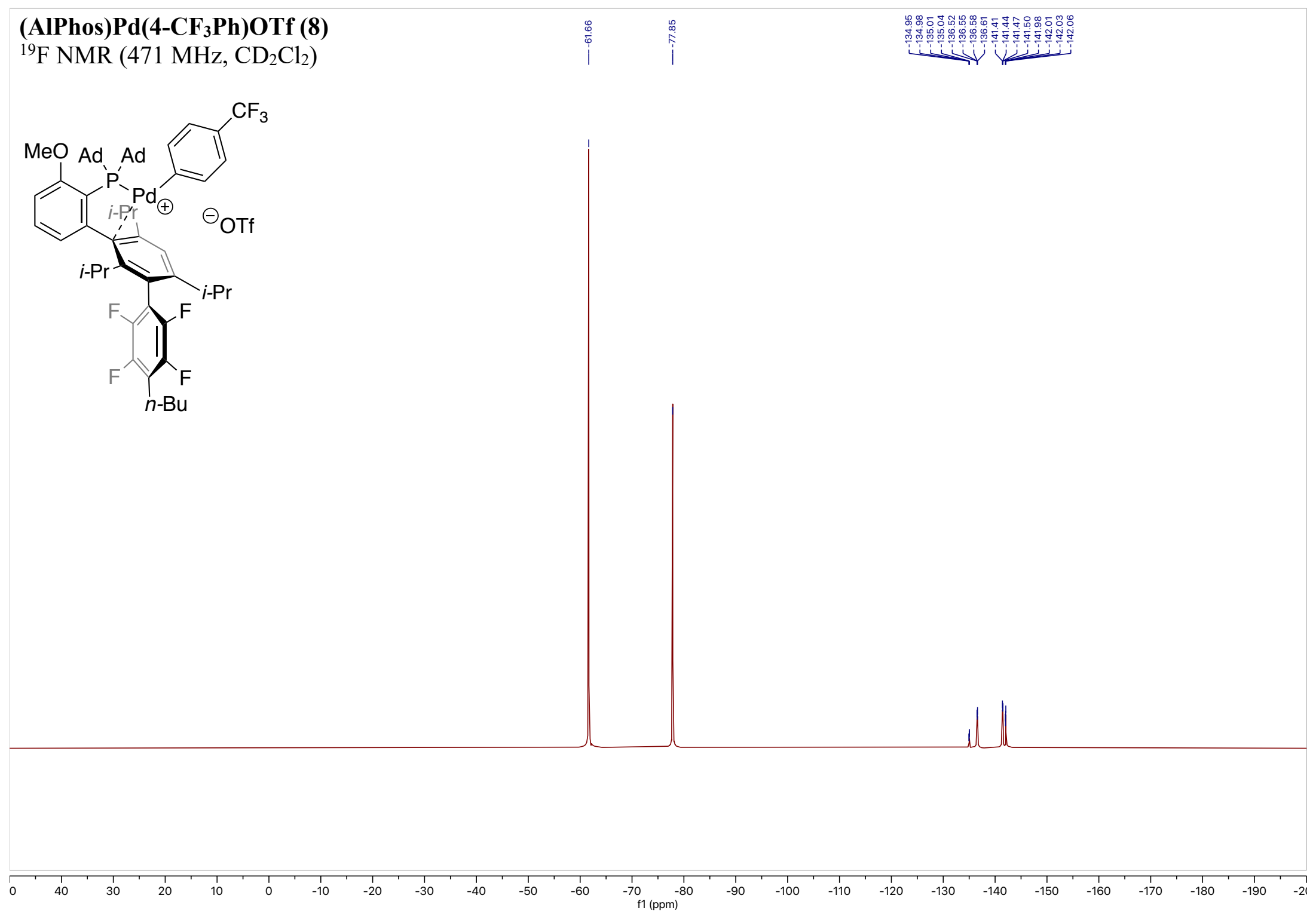




\section{(AIPhos)Pd(4-CF 3 Ph)OTf (8)}

${ }^{31} \mathrm{P}$ NMR (203 MHz, $\mathrm{CD}_{2} \mathrm{Cl}_{2}$ )
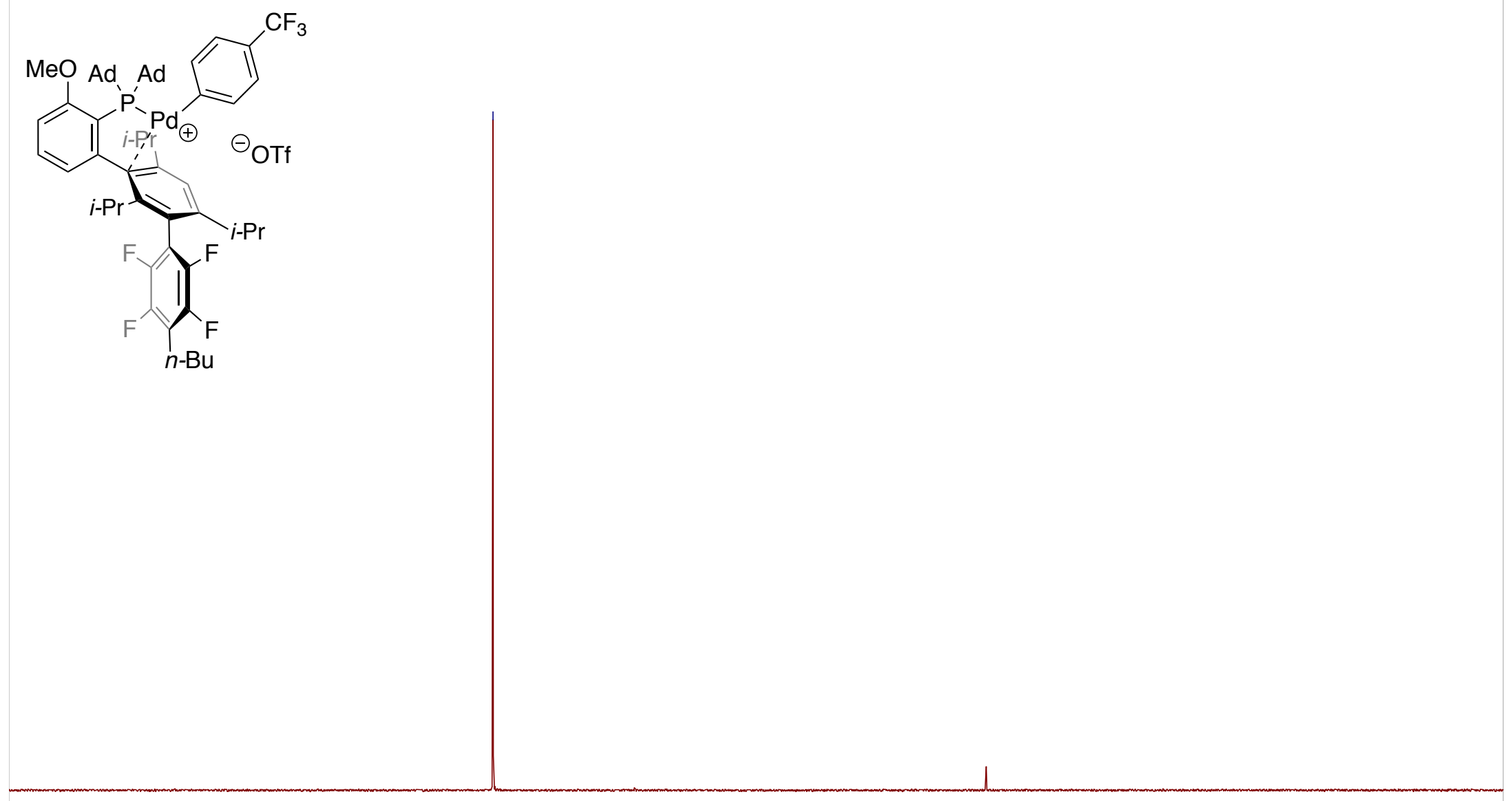


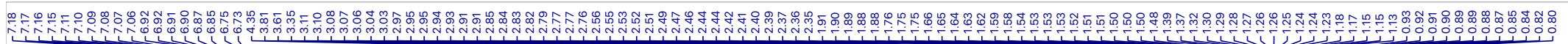

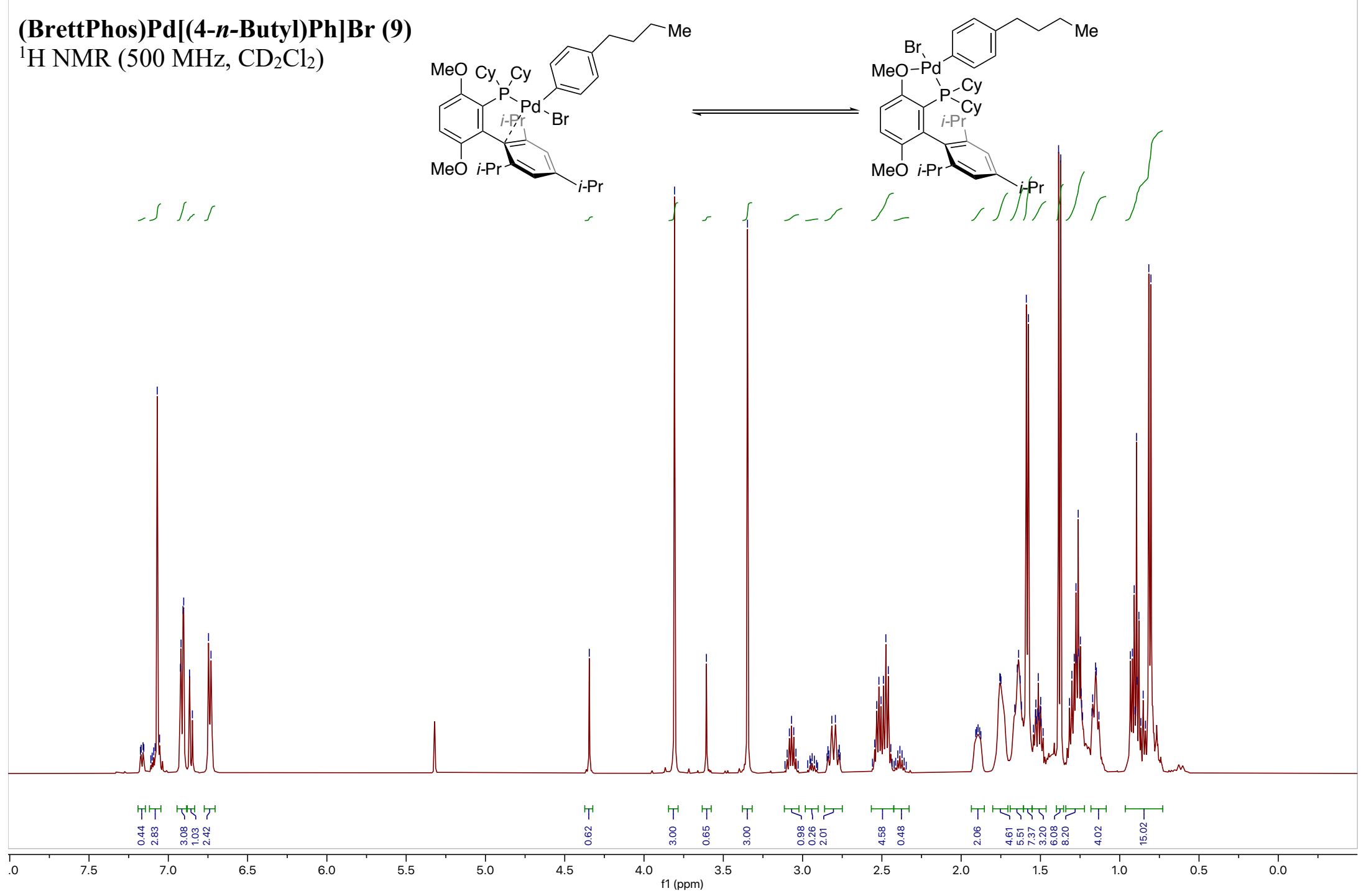









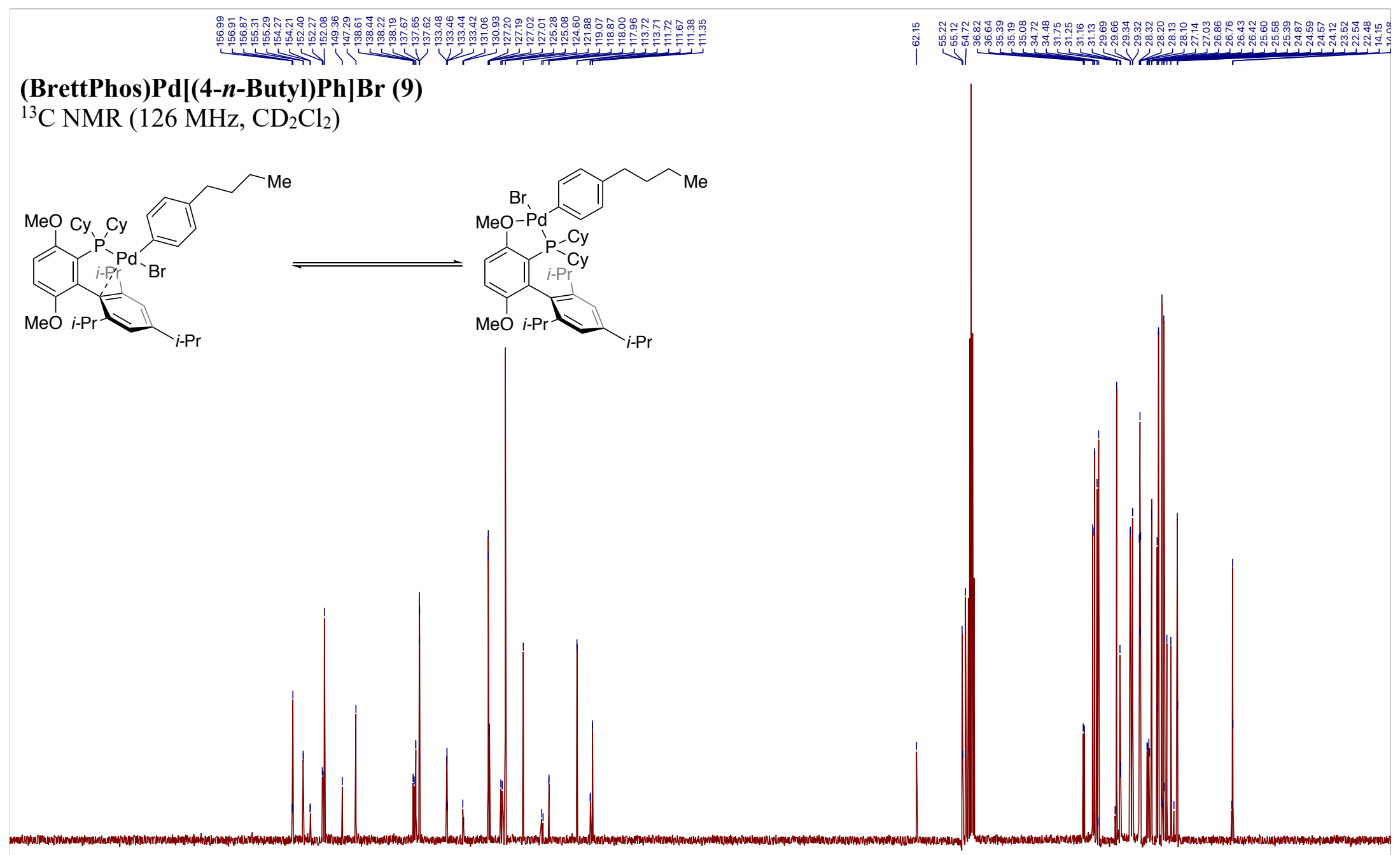




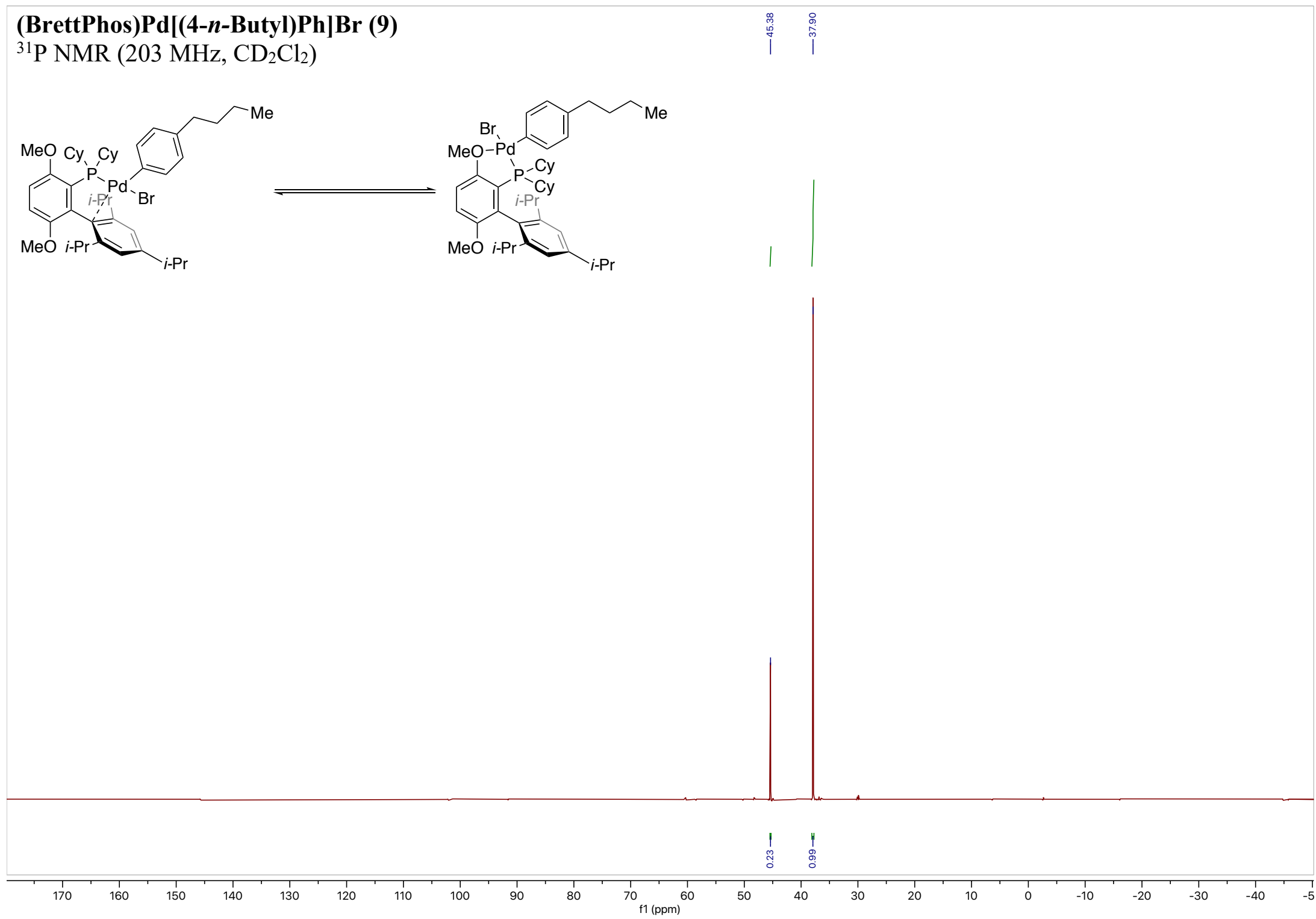




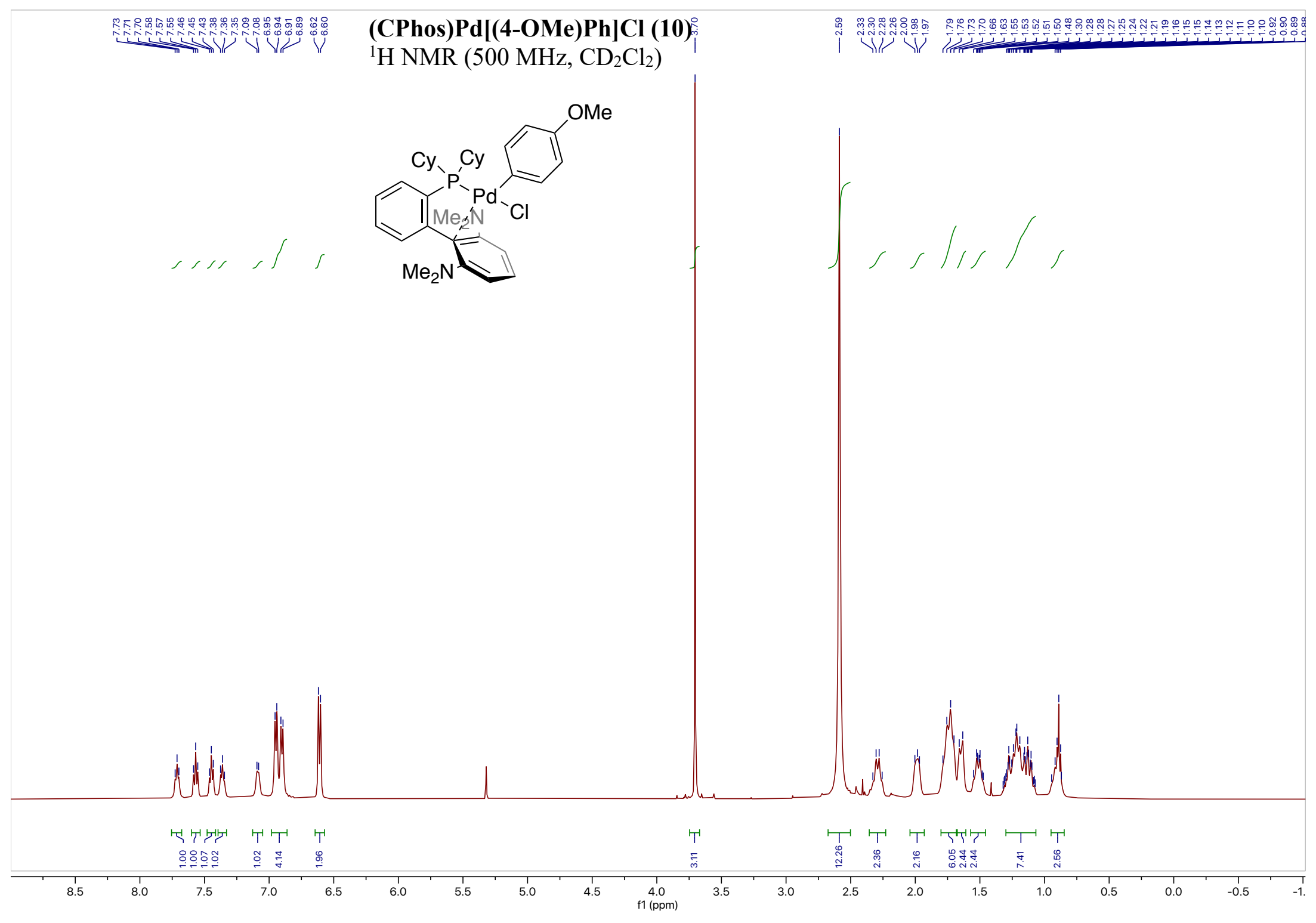




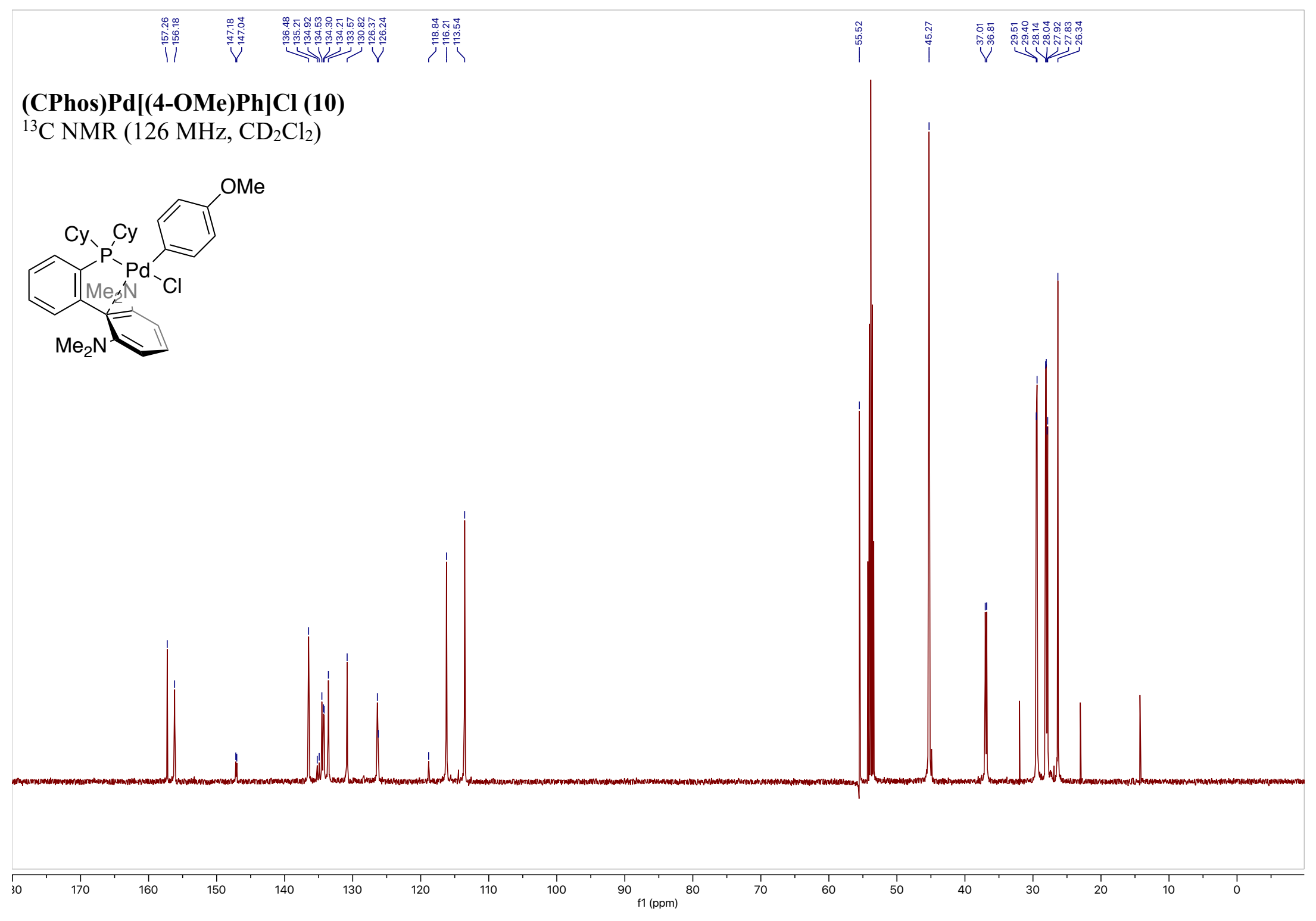


(CPhos)Pd[(4-OMe)Ph]Cl (10)

${ }^{31} \mathrm{P}$ NMR (203 MHz, $\mathrm{CD}_{2} \mathrm{Cl}_{2}$ )

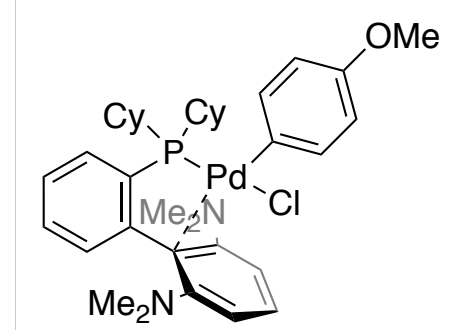




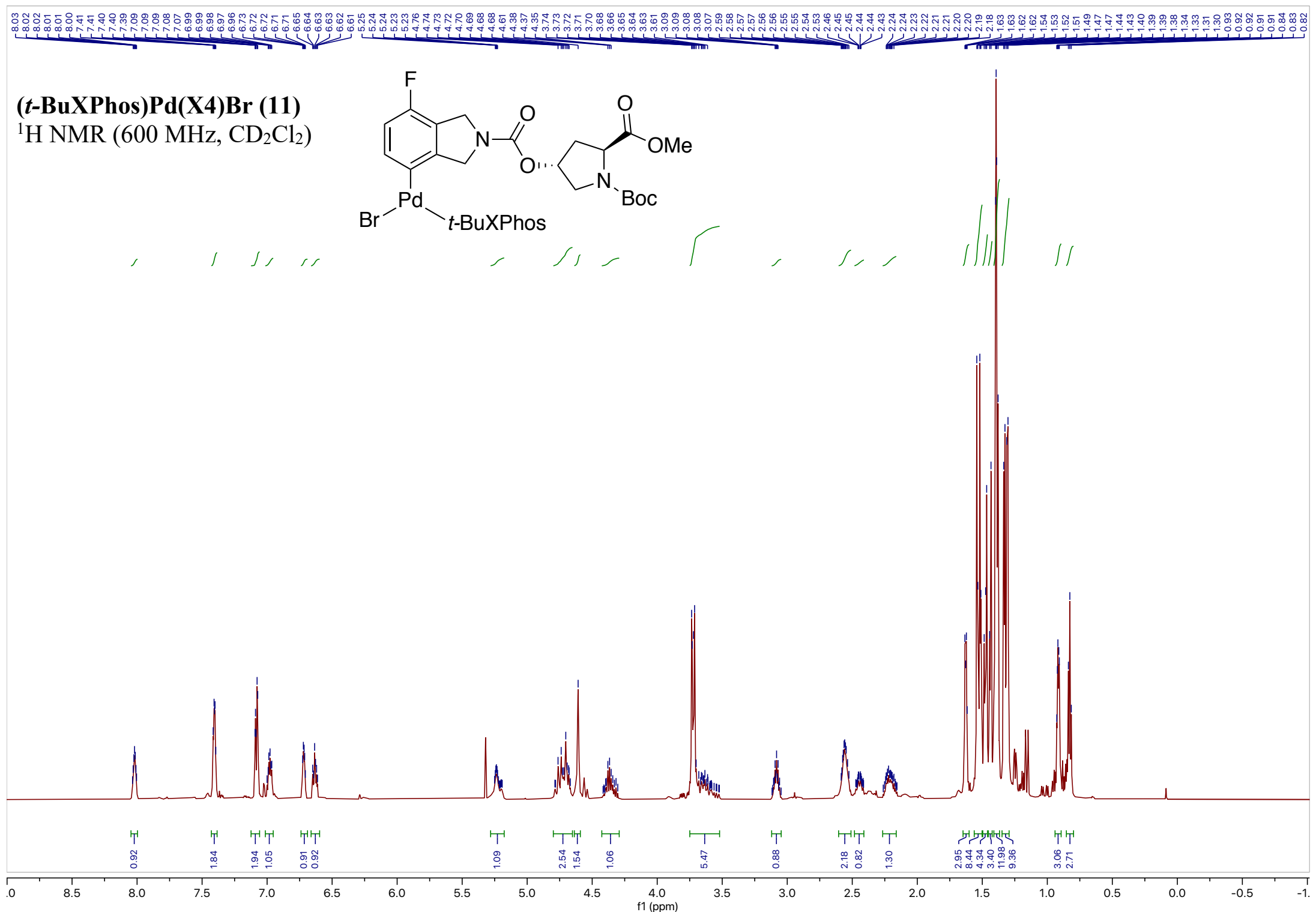




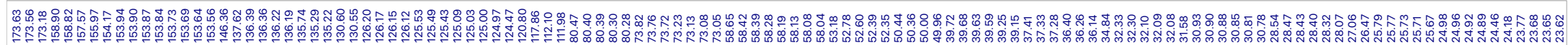

(t-BuXPhos)Pd(X4)Br (11)

${ }^{13} \mathrm{C}$ NMR (151 MHz, $\left.\mathrm{CD}_{2} \mathrm{Cl}_{2}\right)$
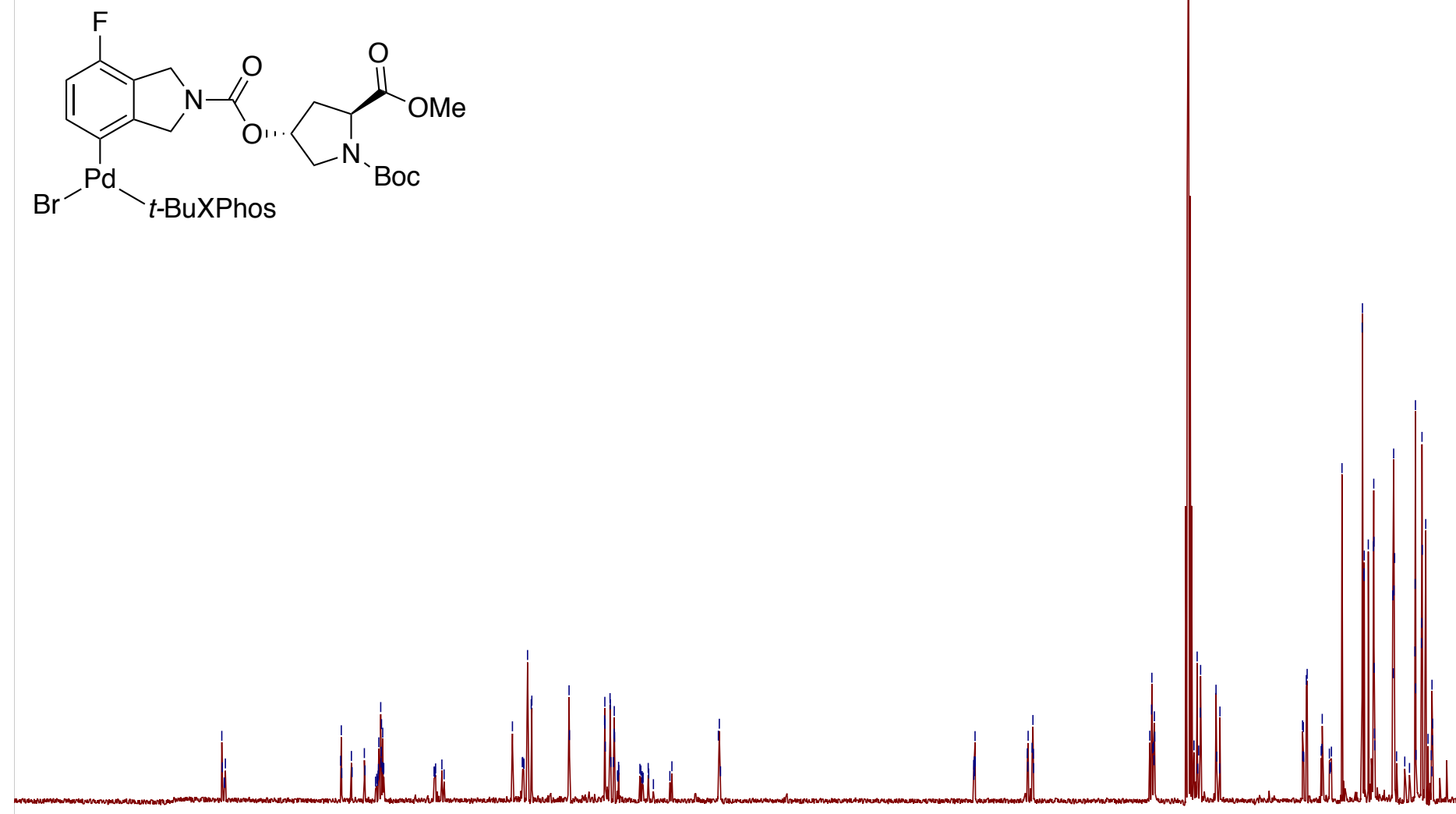
(t-BuXPhos)Pd(X4)Br (11)

${ }^{19} \mathrm{~F}$ NMR (565 MHz, $\left.\mathrm{CD}_{2} \mathrm{Cl}_{2}\right)$
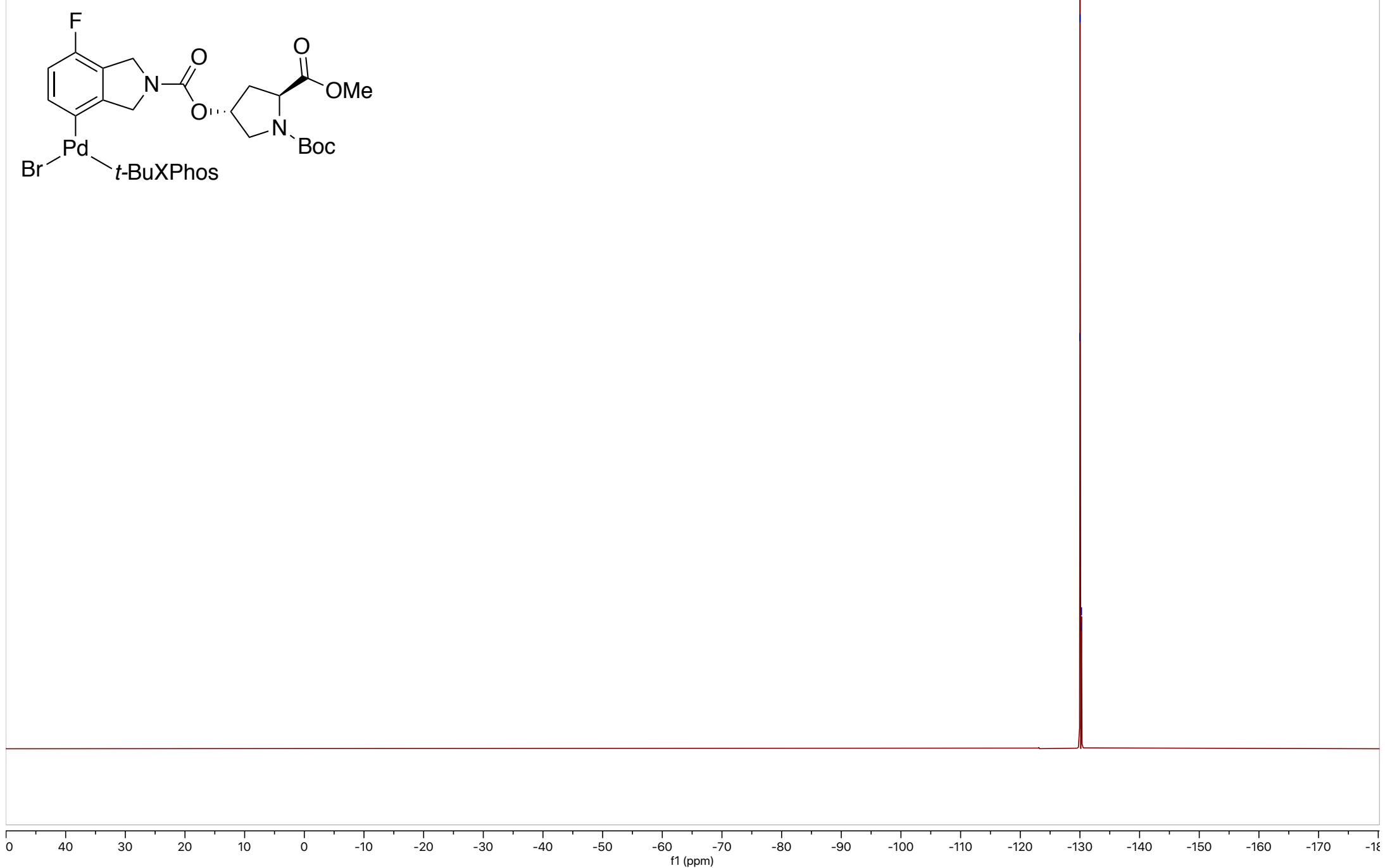


\section{(t-BuXPhos)Pd(X4)Br (11)}

${ }^{31} \mathrm{P}$ NMR (203 MHz, $\mathrm{CD}_{2} \mathrm{Cl}_{2}$ )

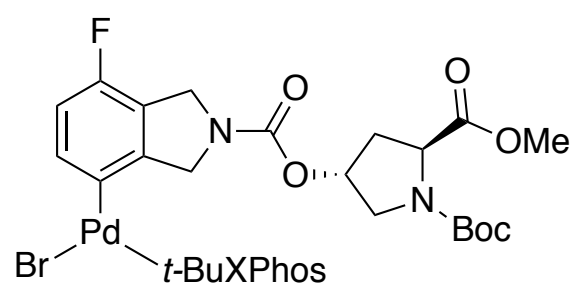

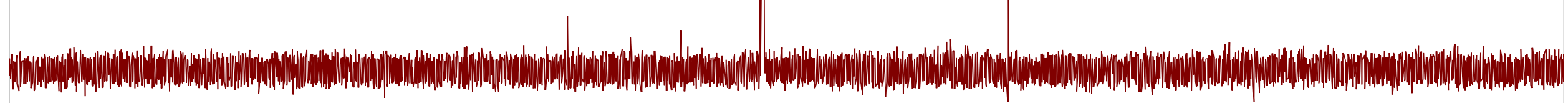

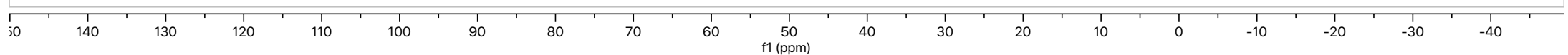




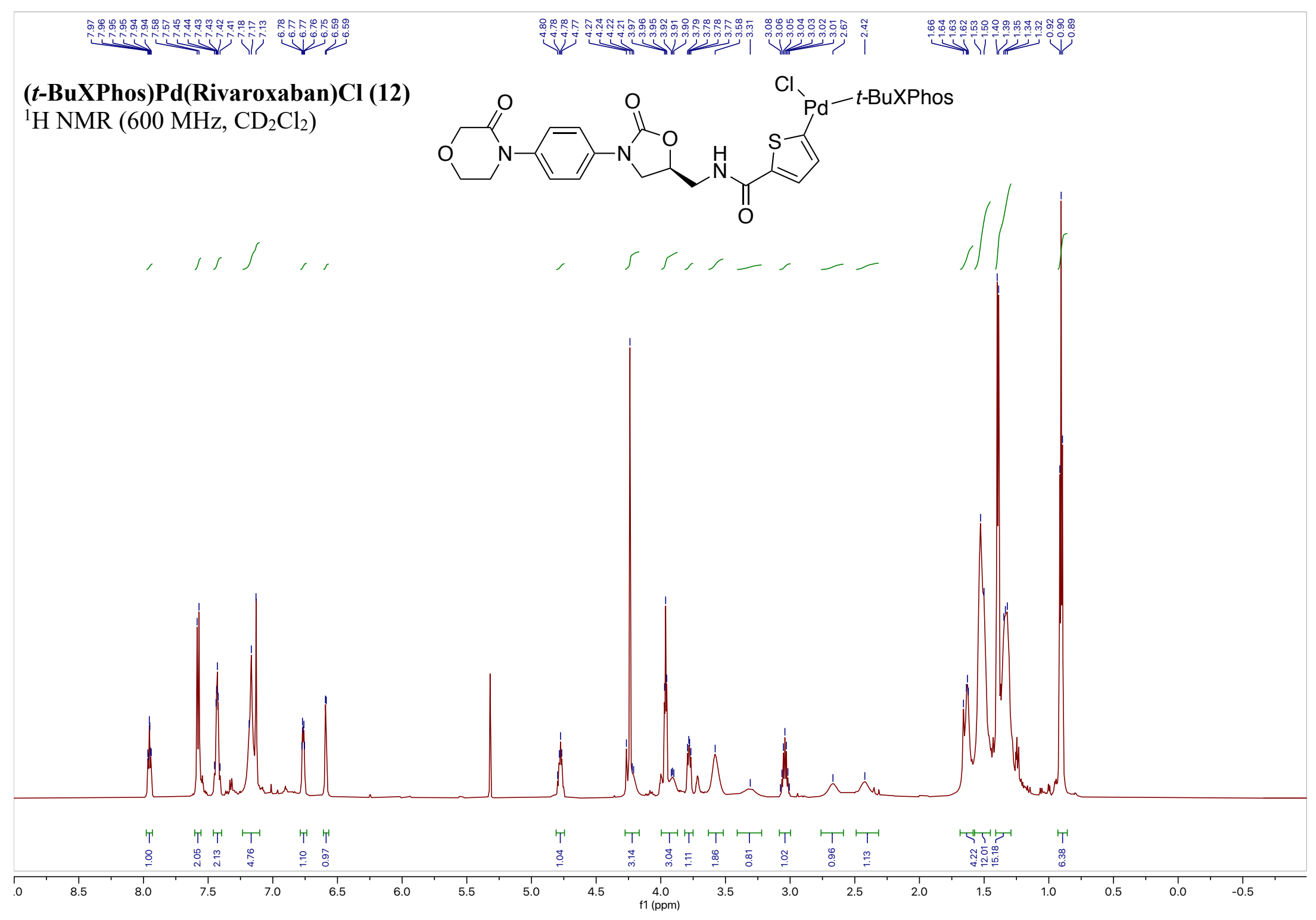




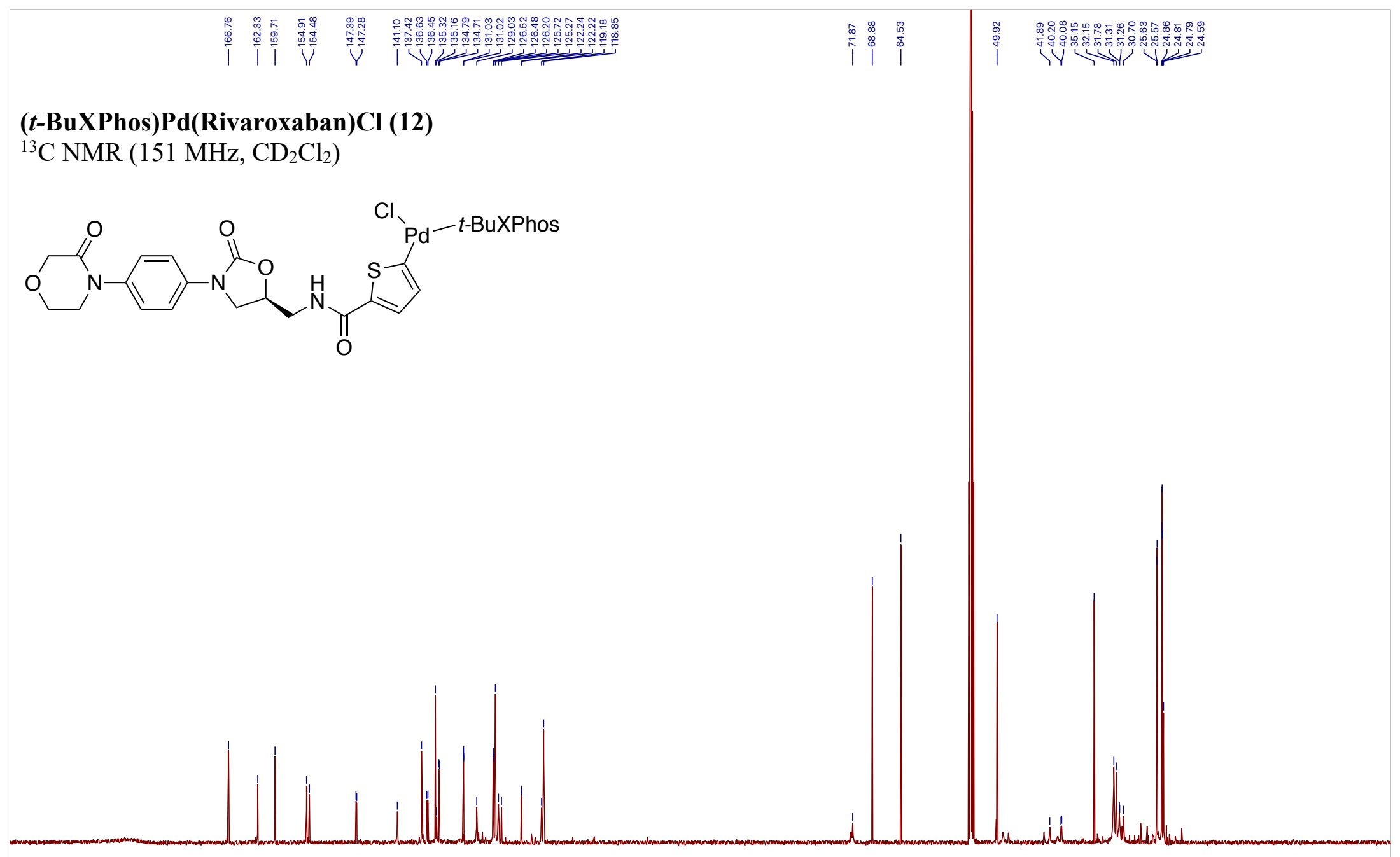




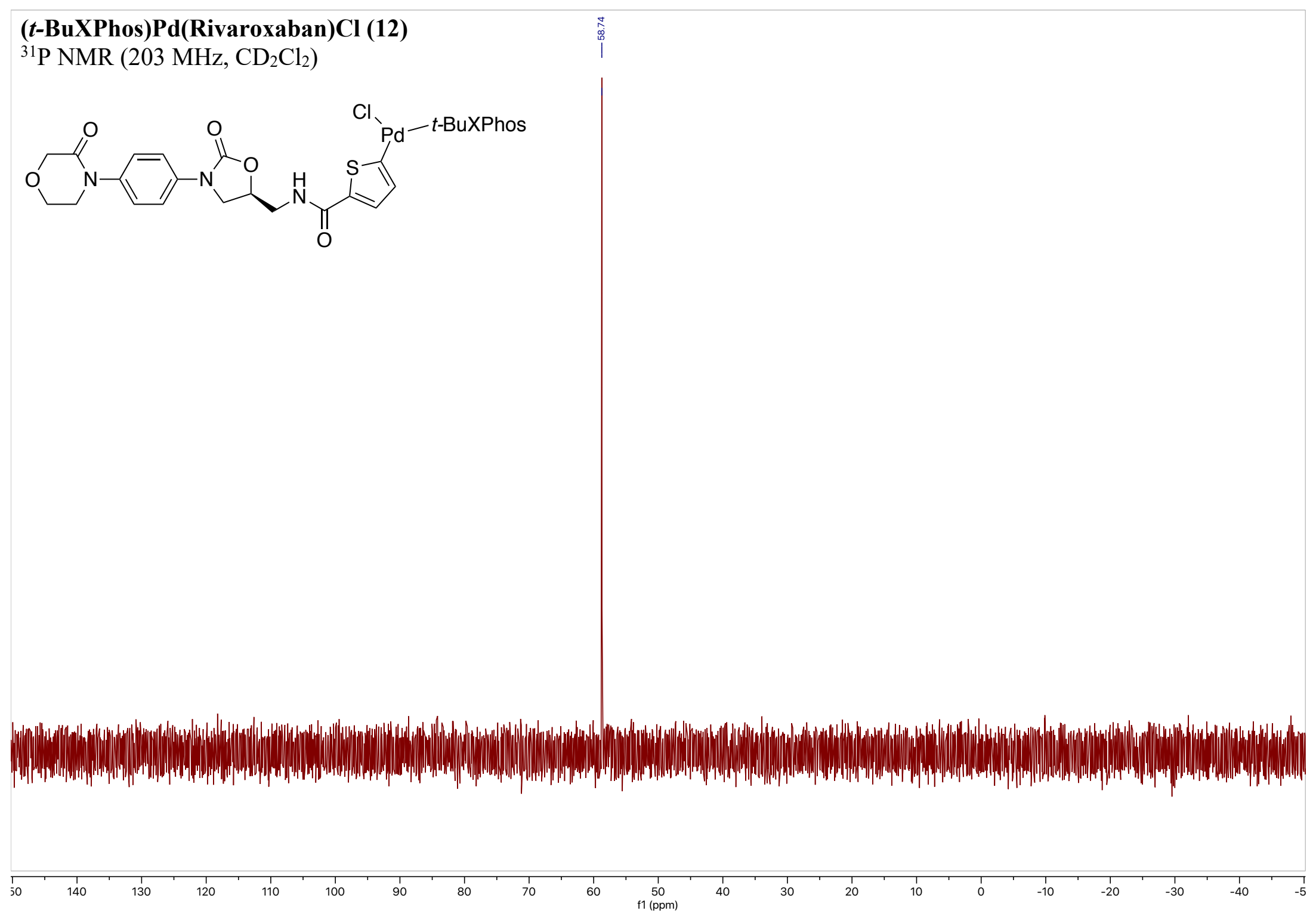




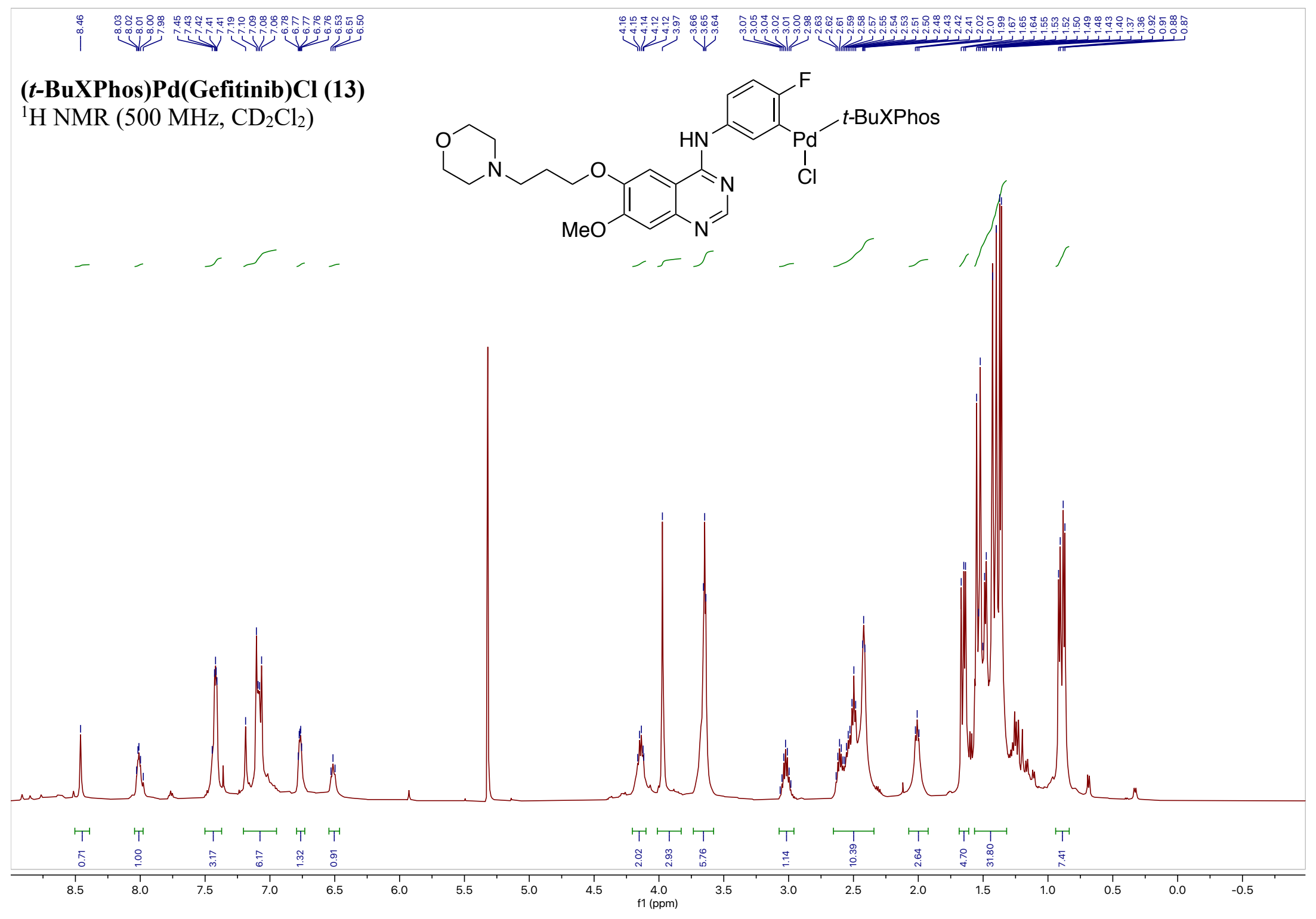




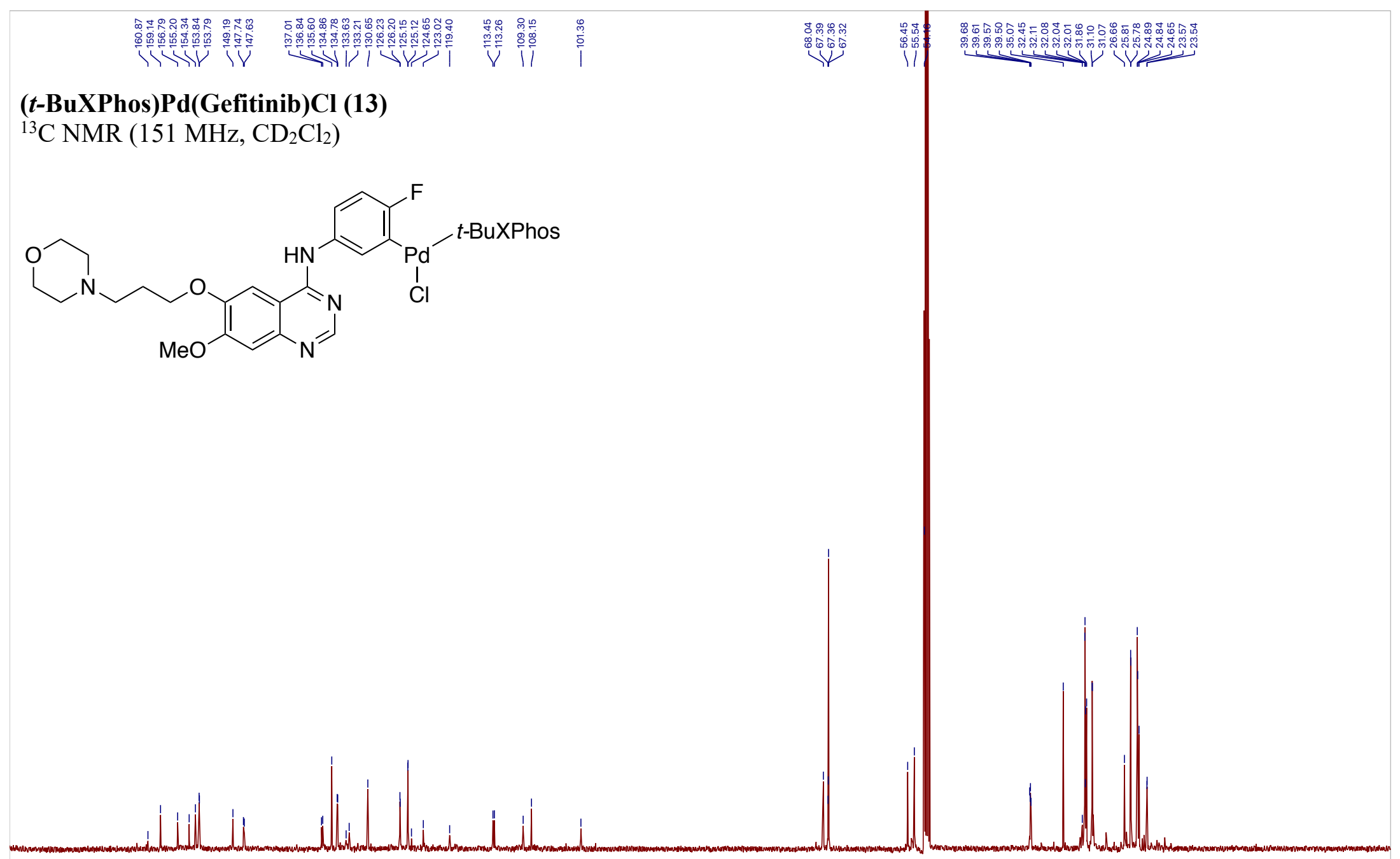









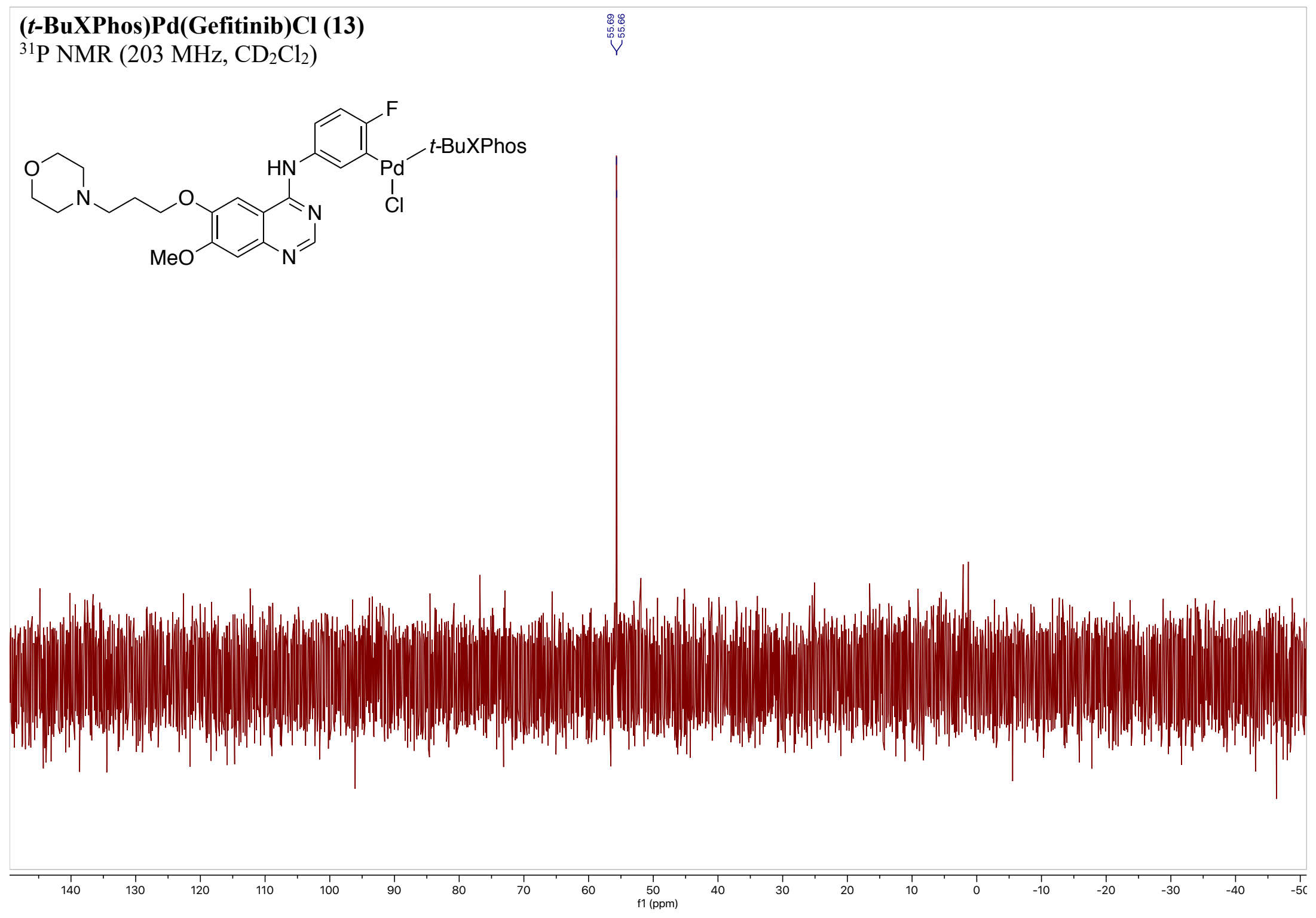




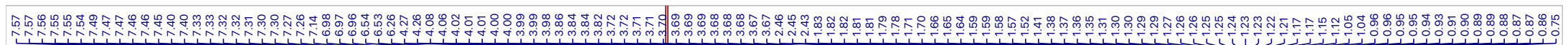

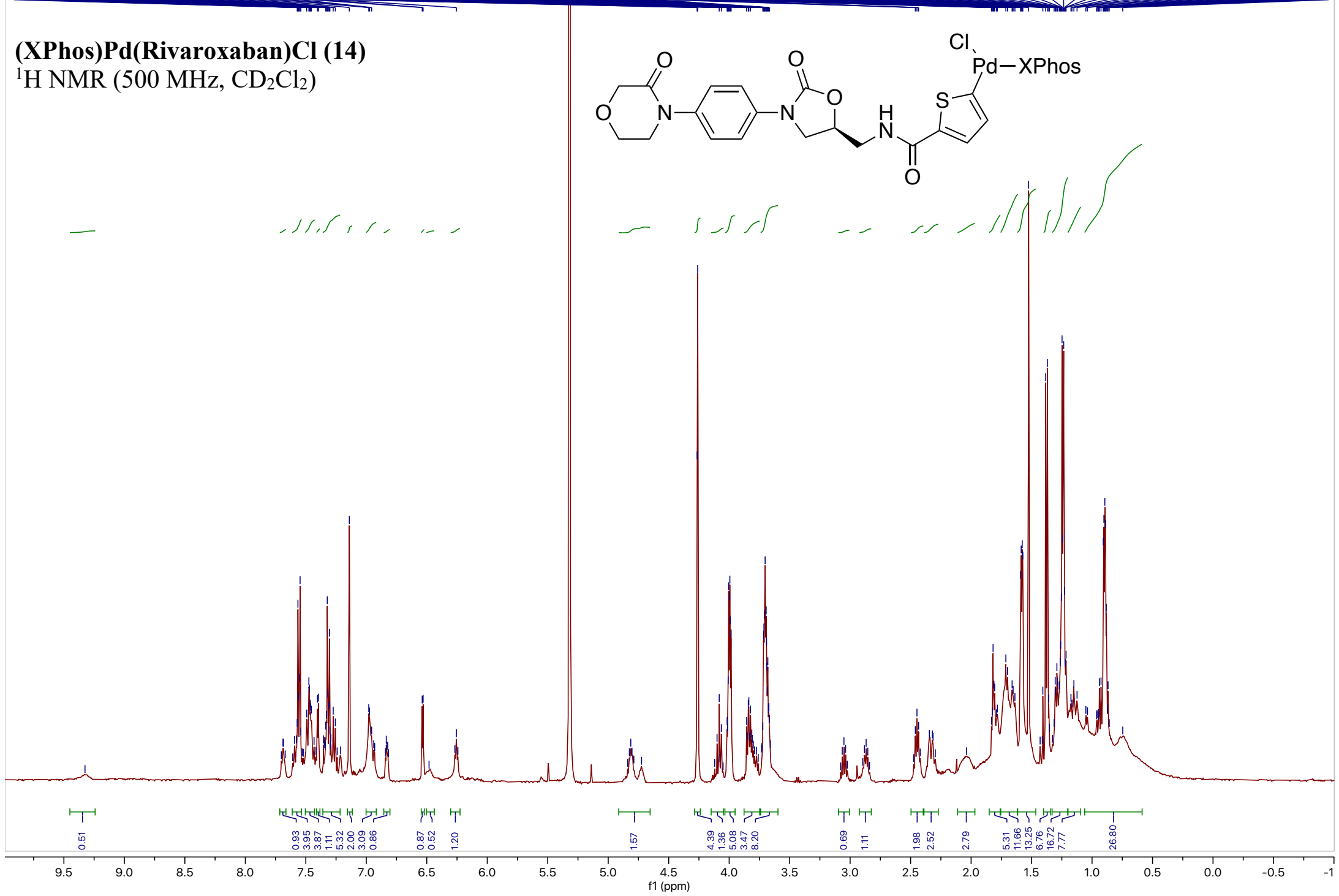




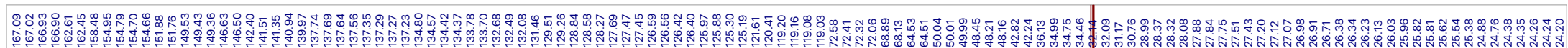
(XPhos)Pd(Rivaroxaban)Cl (14)

${ }^{13} \mathrm{C}$ NMR $\left(151 \mathrm{MHz}, \mathrm{CD}_{2} \mathrm{Cl}_{2}\right)$<smiles>[H][Y10]([H])([H])[R](Cl)c1ccc(C(=O)NC[C@H]2CN(c3ccc(N4CCOCC4=O)cc3)C(=O)O2)s1</smiles>



$190 \quad 180,170,160$
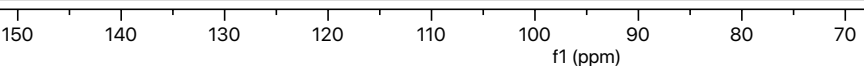

60






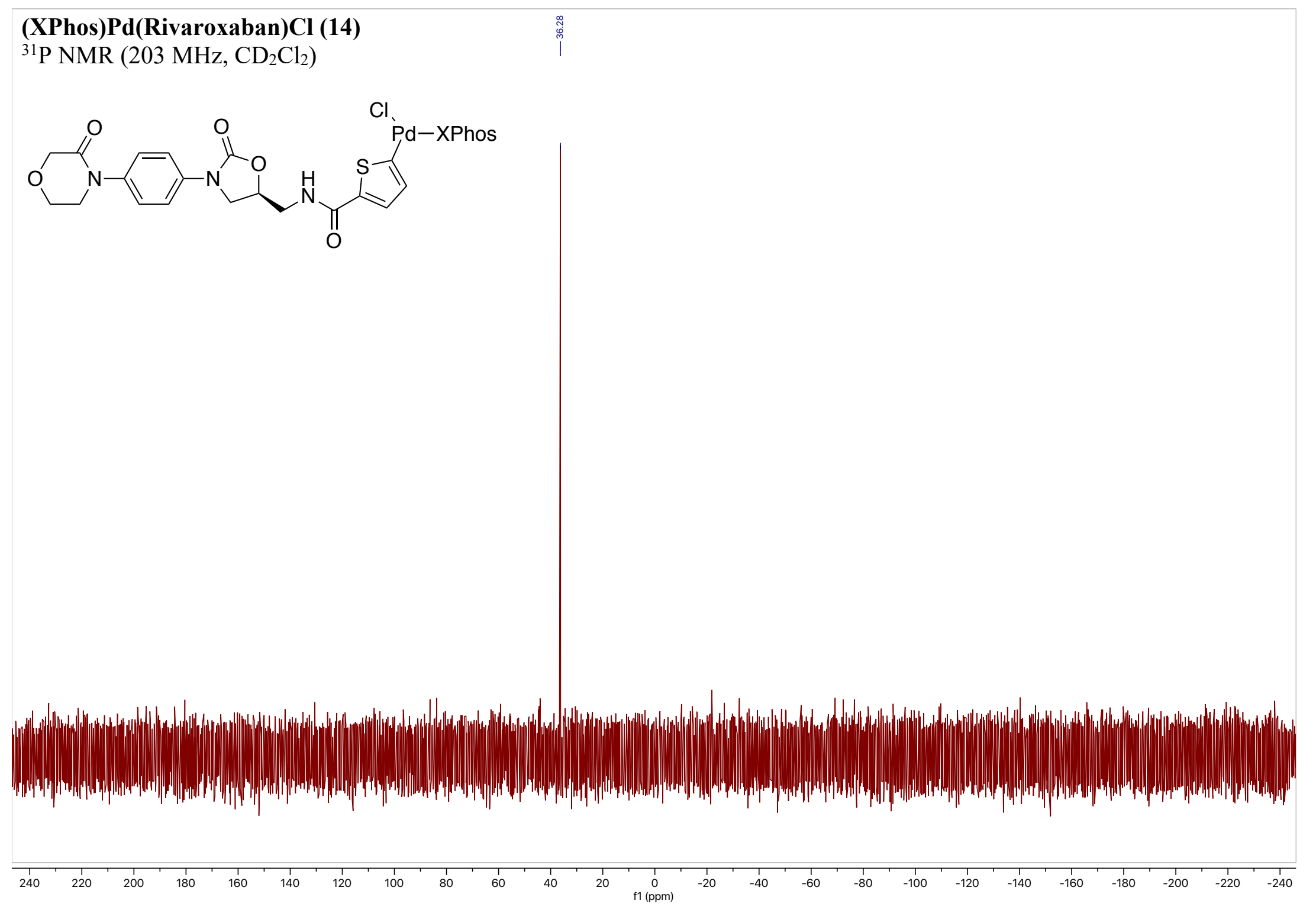




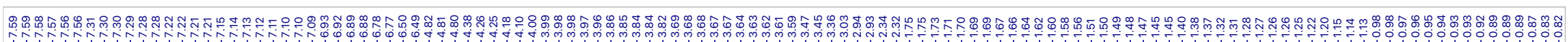

(BrettPhos)Pd(Rivaroxaban)Cl (15)

${ }^{1} \mathrm{H}$ NMR $\left(500 \mathrm{MHz}, \mathrm{CD}_{2} \mathrm{Cl}_{2}\right)$<smiles>O=C(NCC1CN(c2ccc(N3CCOCC3=O)cc2)C(=O)O1)c1ccc(Br)s1</smiles>
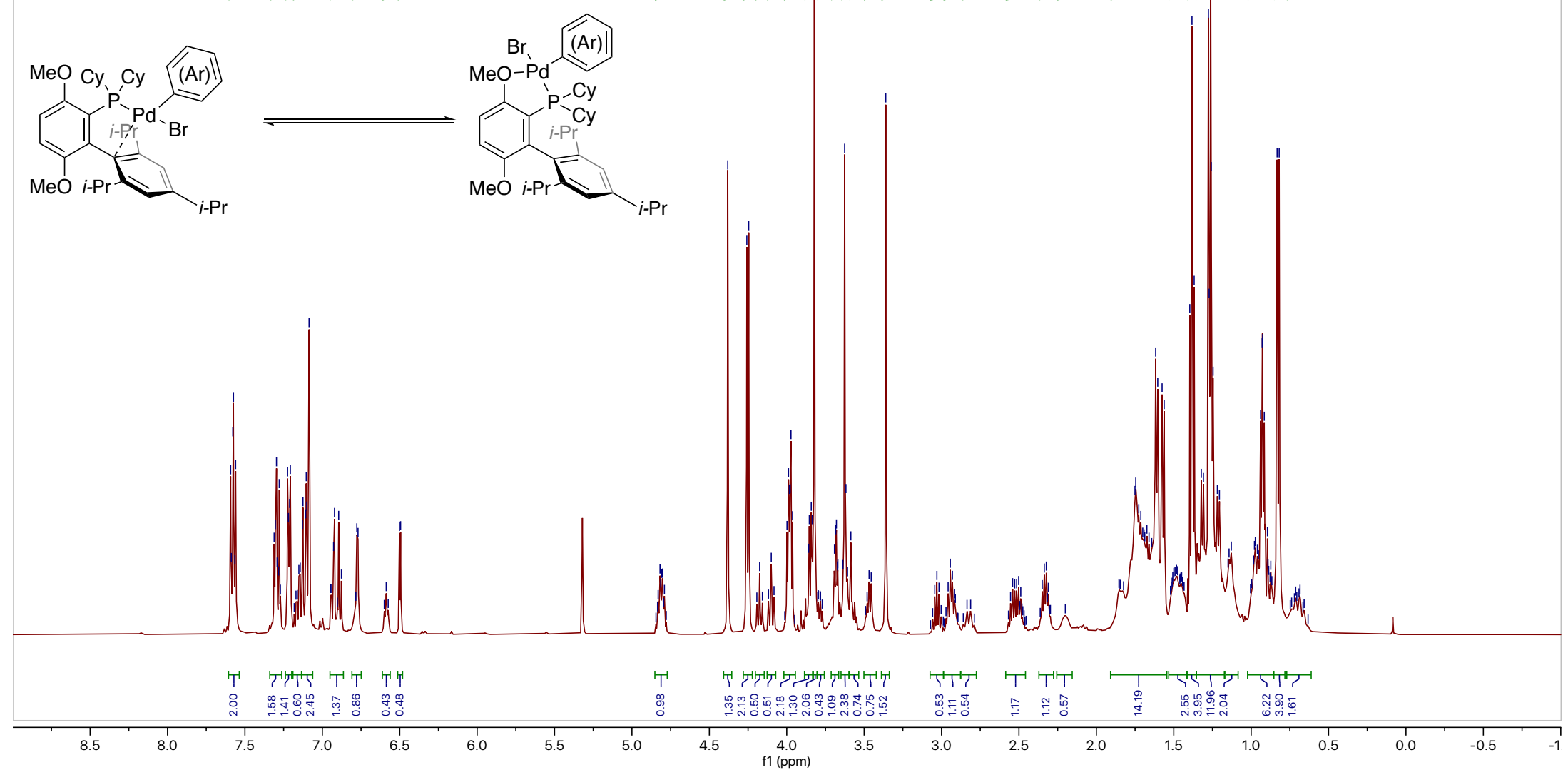


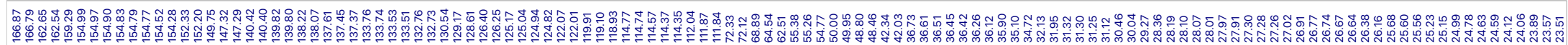
- . - . -

\section{(BrettPhos)Pd(Rivaroxaban)Cl (15)}

${ }^{13} \mathrm{C}$ NMR $\left(126 \mathrm{MHz}, \mathrm{CD}_{2} \mathrm{Cl}_{2}\right)$



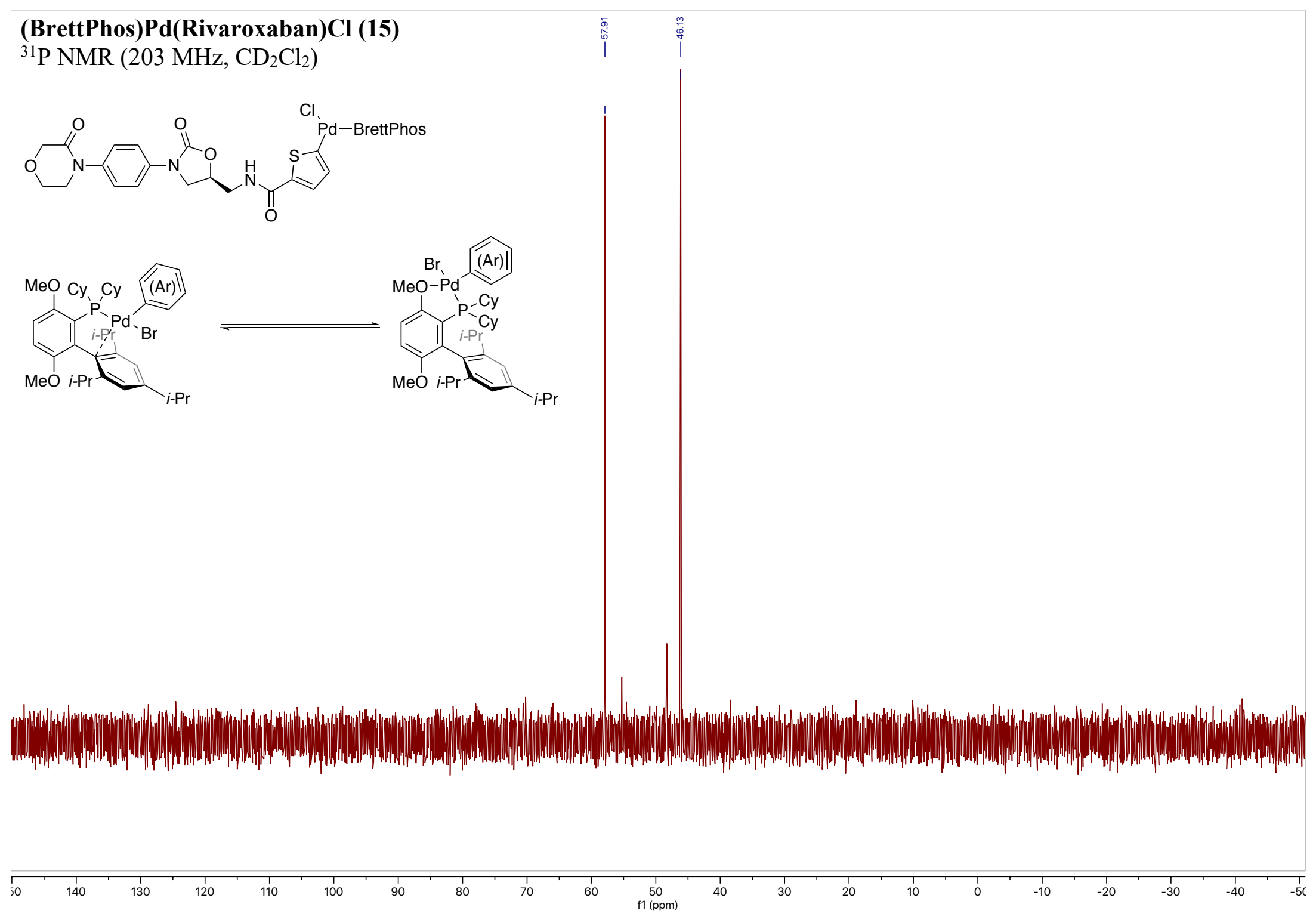


卉

(RuPhos)Pd(2-Et-6-Me-3-pyridyl)OTf (16)

${ }^{1} \mathrm{H}$ NMR (500 MHz, $\left.\mathrm{CD}_{3} \mathrm{CN}\right)$
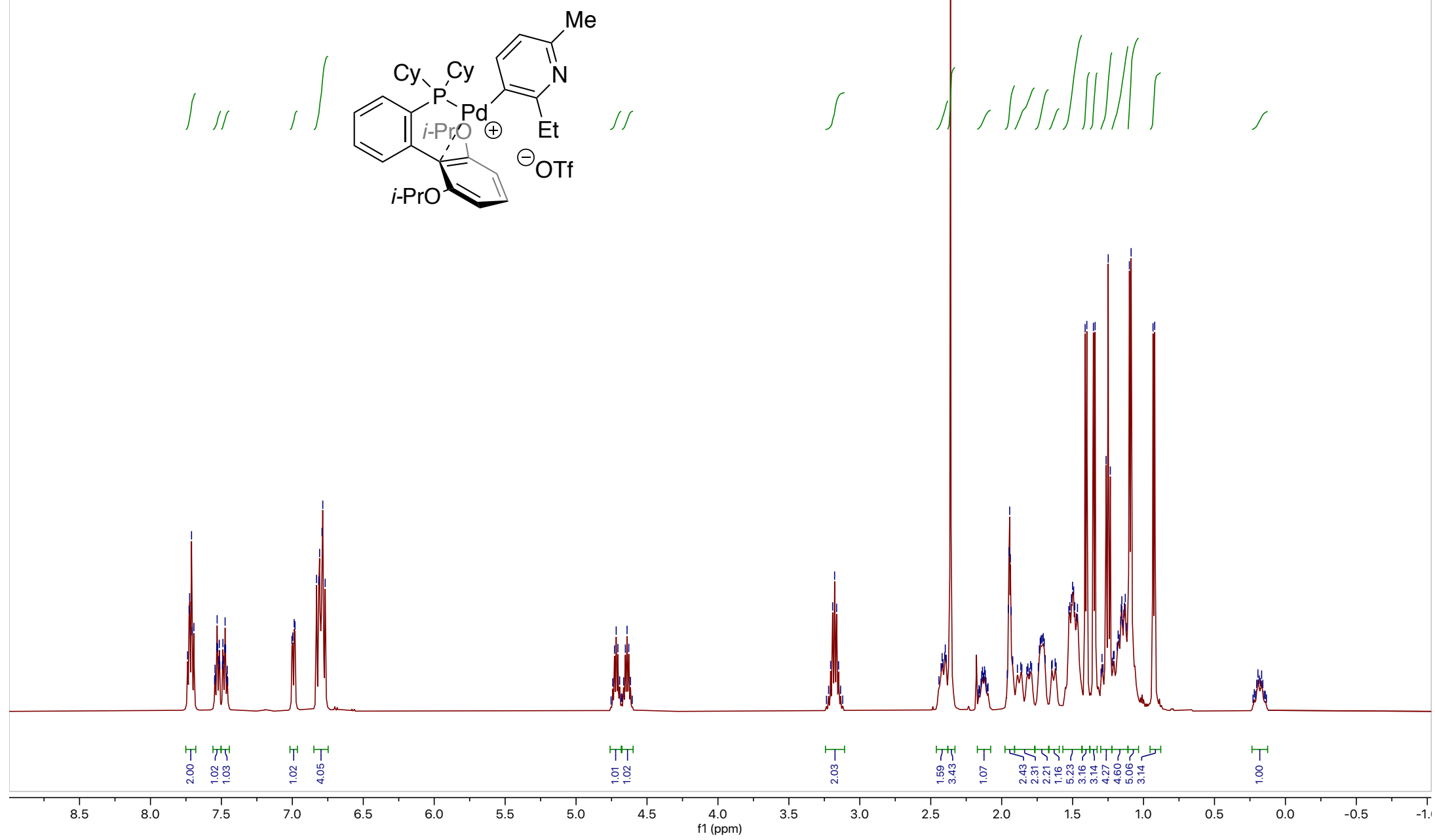


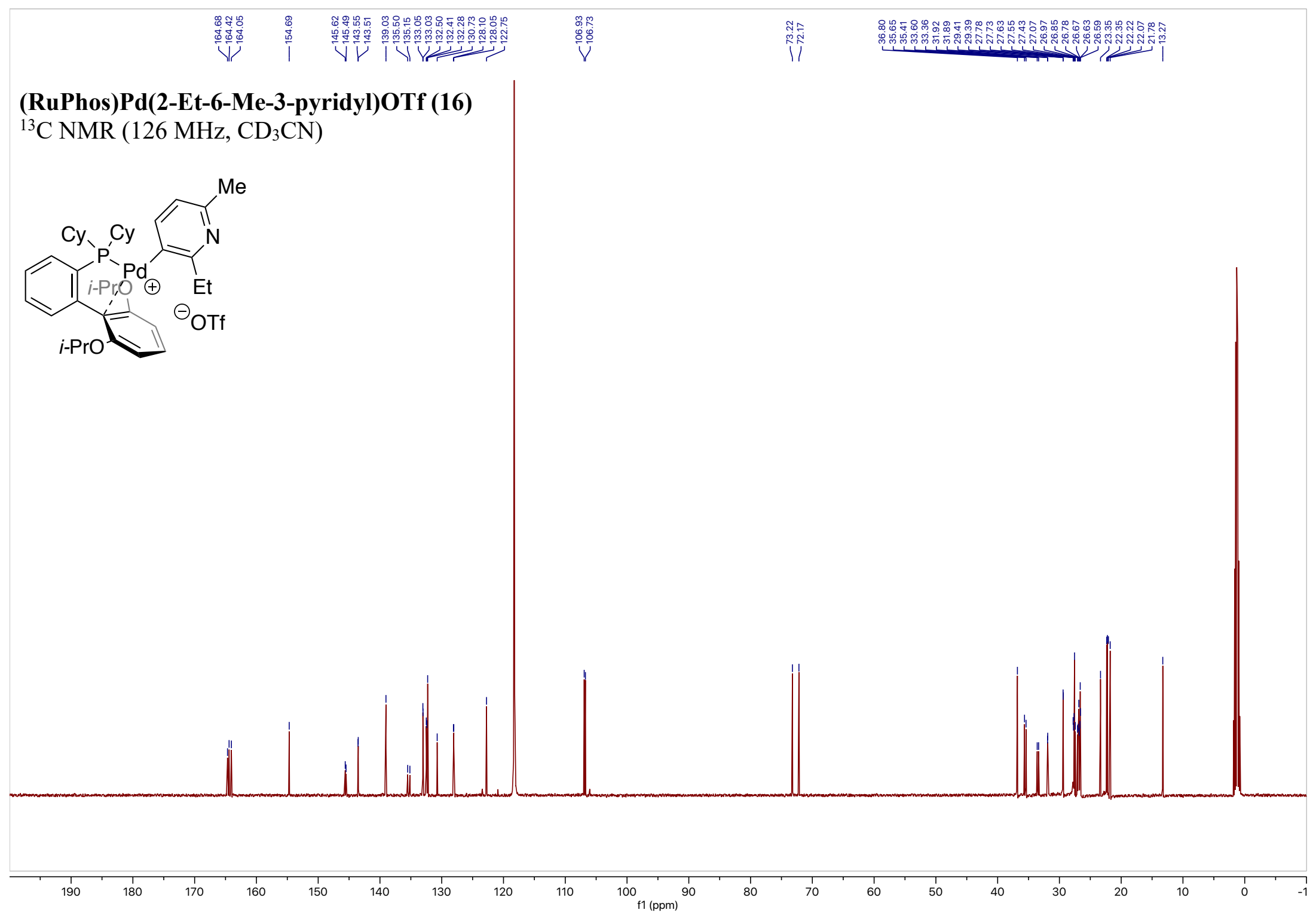




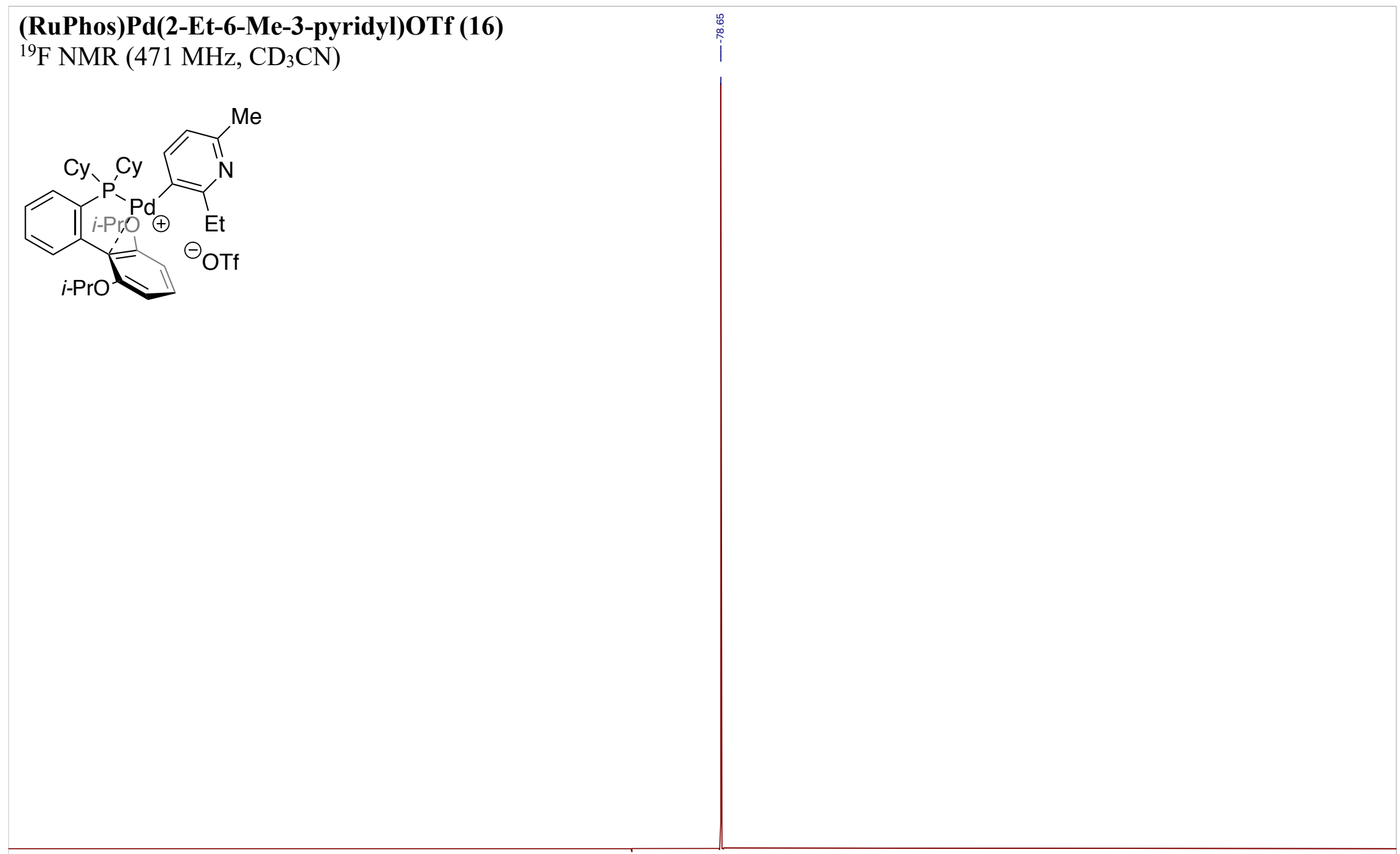

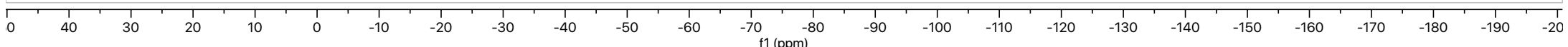


(RuPhos)Pd(2-Et-6-Me-3-pyridyl)OTf (16)

${ }^{31} \mathrm{P}$ NMR (203 MHz, CD 3 CN)

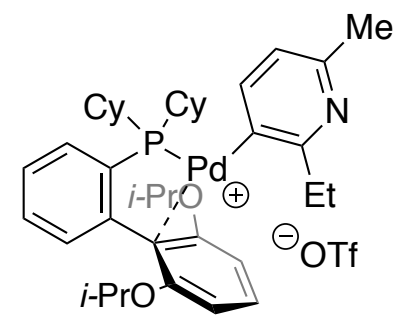


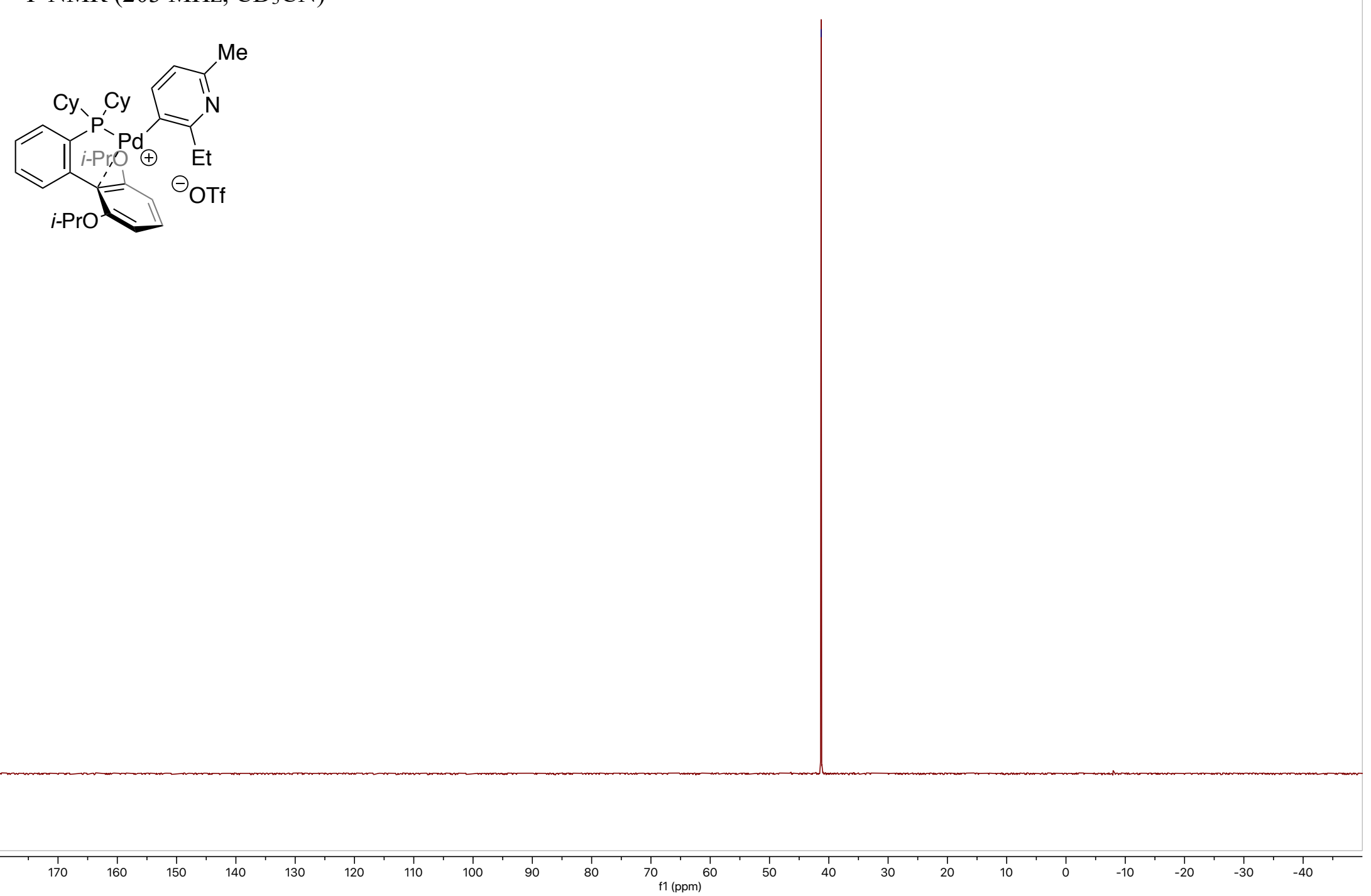

S114 


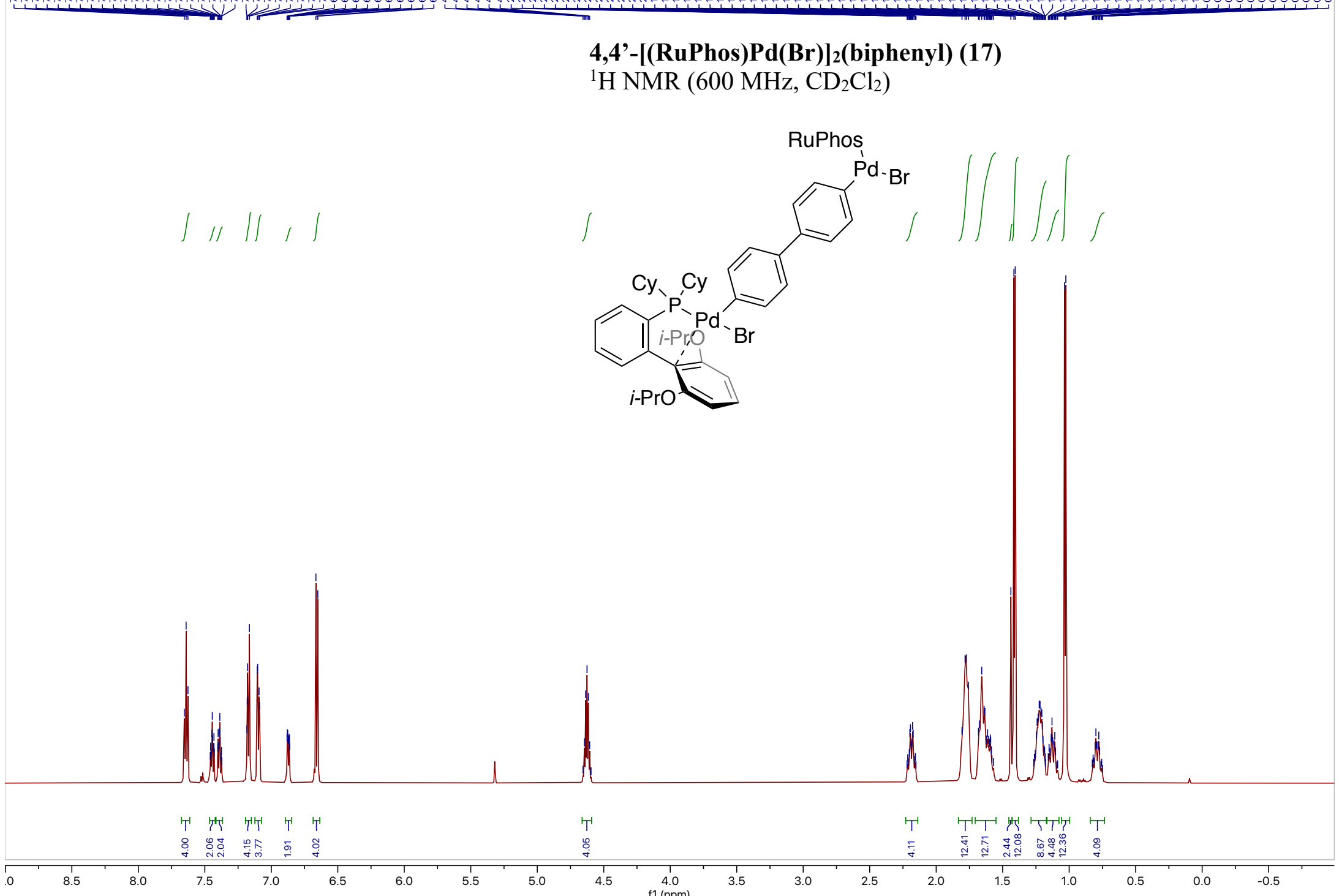




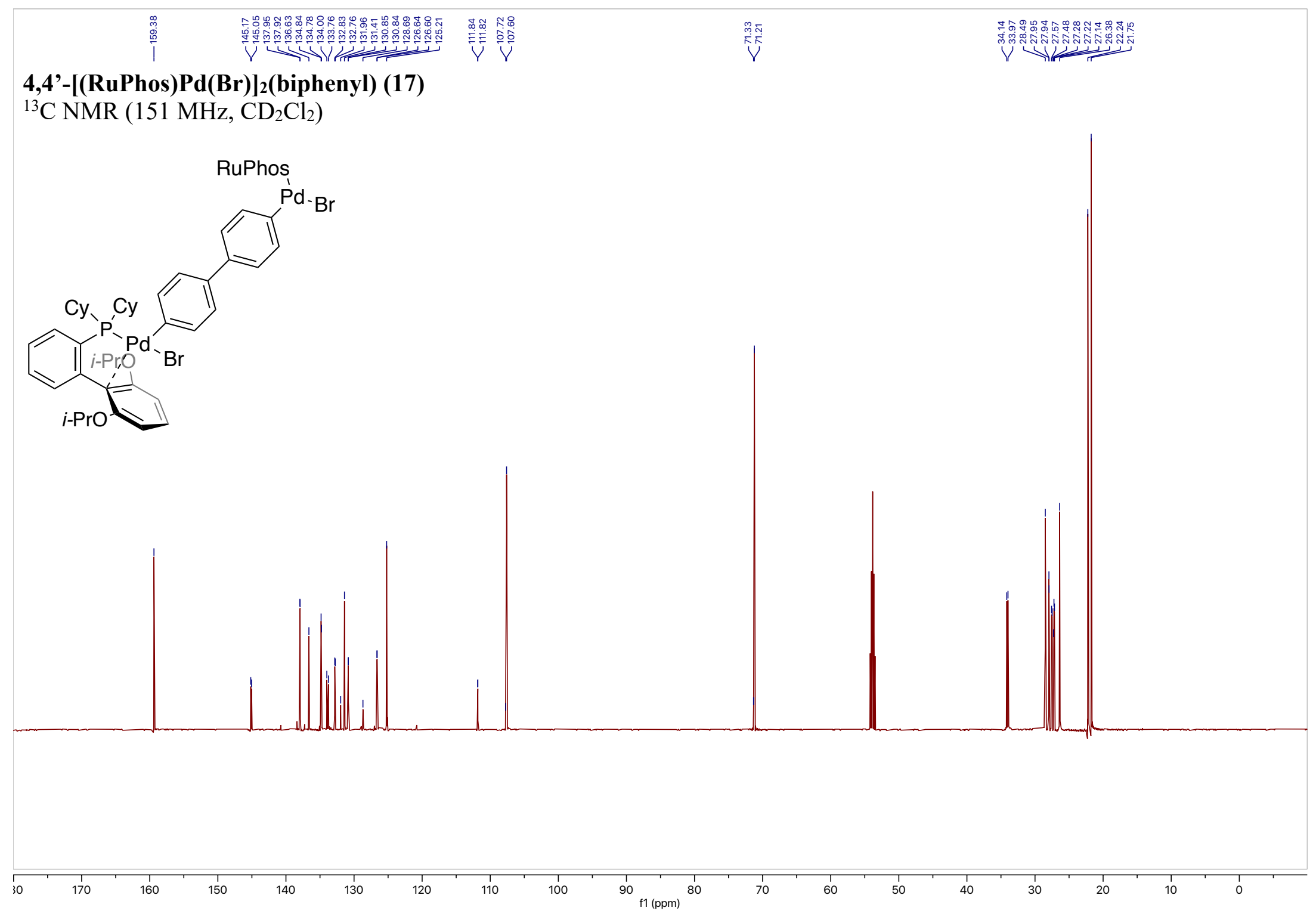


4,4'-[(RuPhos)Pd(Br) $]_{2}$ (biphenyl) (17)

${ }^{31} \mathrm{P}$ NMR (203 MHz, $\mathrm{CD}_{2} \mathrm{Cl}_{2}$ )

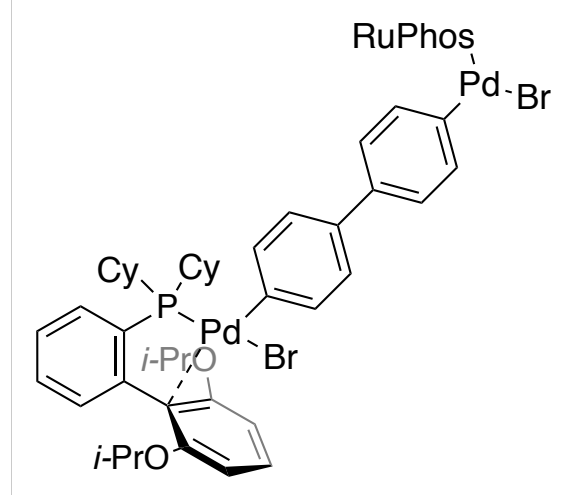

\begin{tabular}{c|c|c|c|c|}
\hline 50 & 140 & 130 & 120 & 110
\end{tabular}

100 90 80 60 f1 (ppm) 


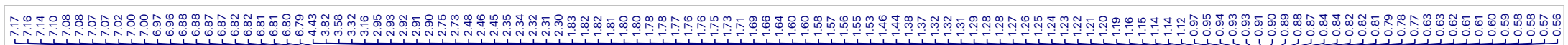

\section{(BrettPhos)Pd(4-I-Ph)I (18)}

${ }^{1} \mathrm{H}$ NMR $\left(600 \mathrm{MHz}, \mathrm{CDCl}_{3}\right)$
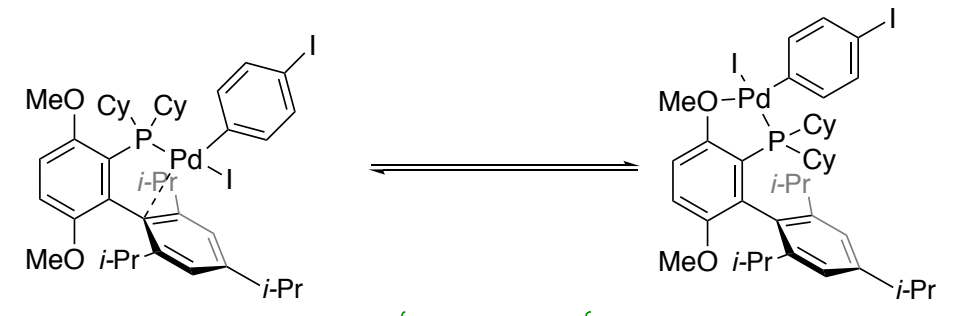

$1.81 / n$
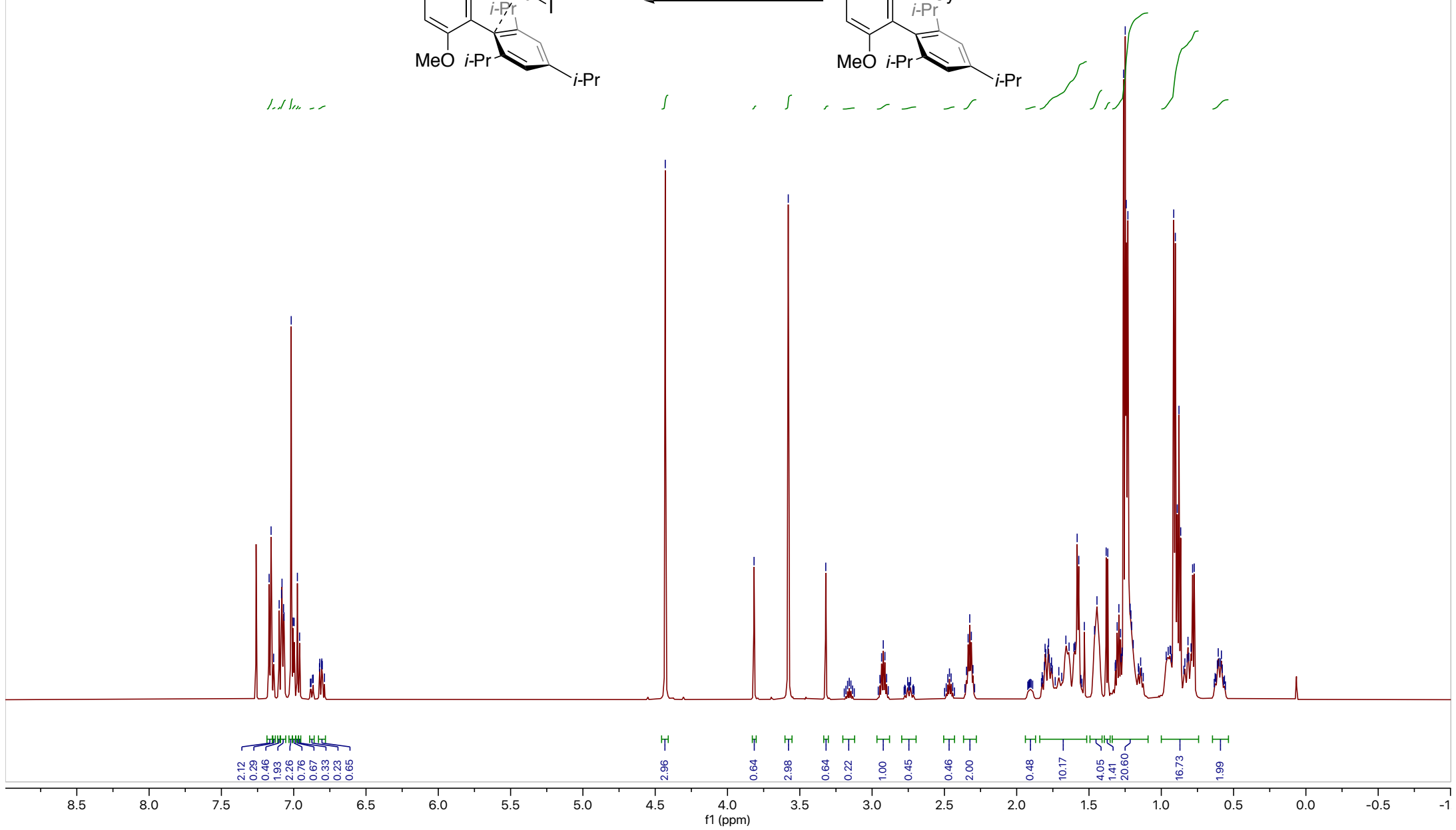

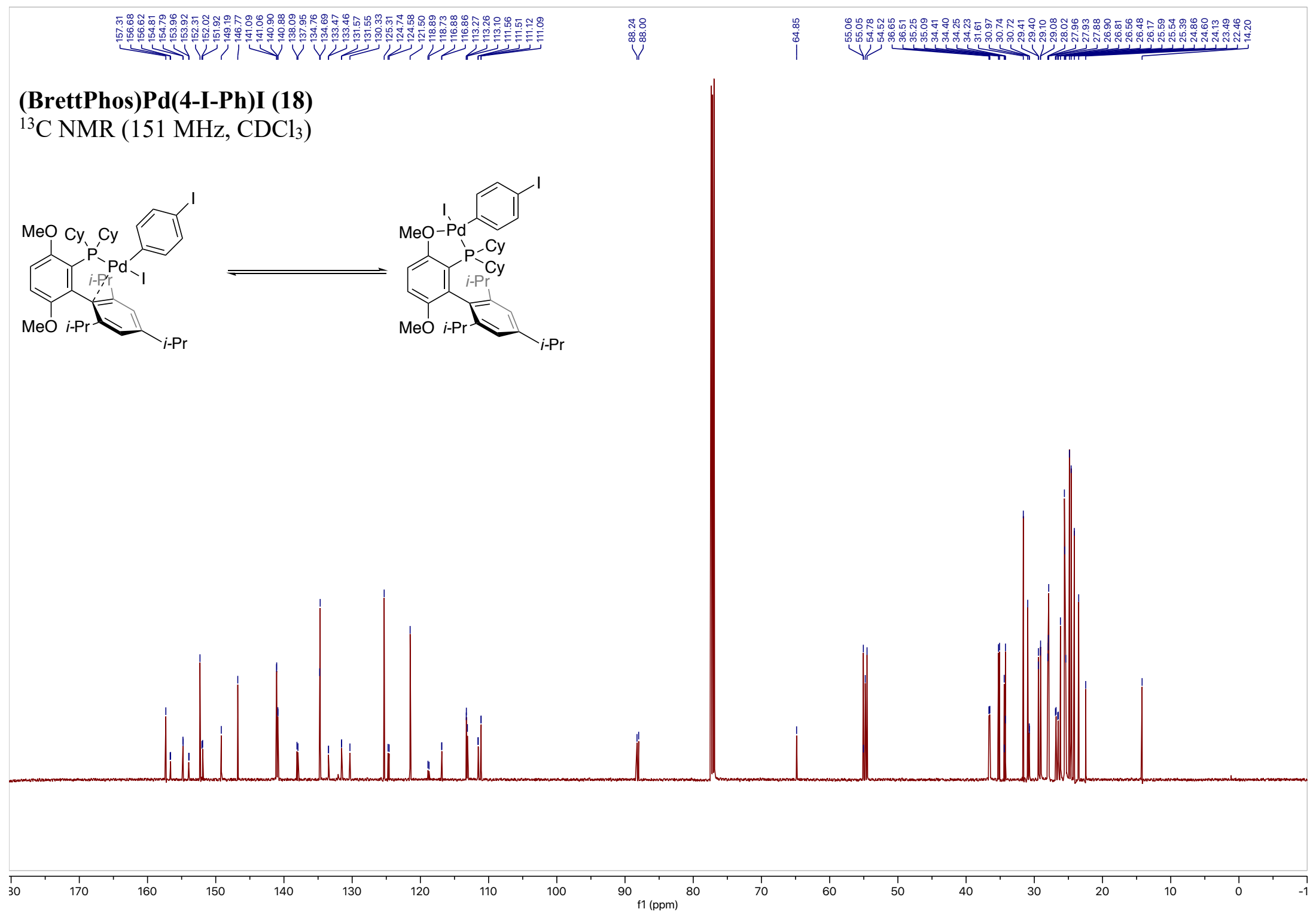
(BrettPhos)Pd(4-I-Ph)I (18)

${ }^{31} \mathrm{P}$ NMR (203 MHz, $\mathrm{CDCl}_{3}$ )

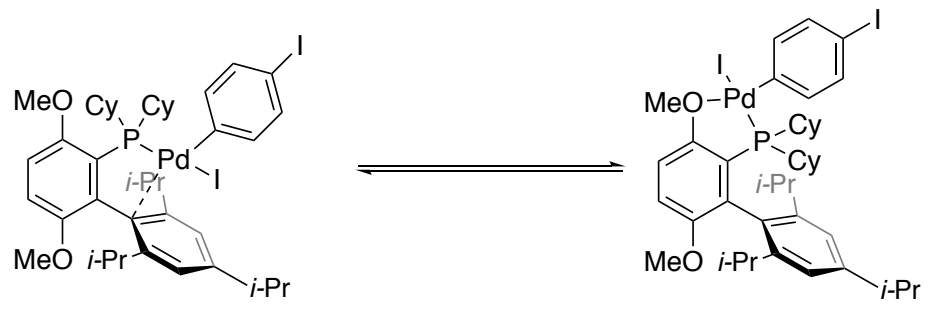

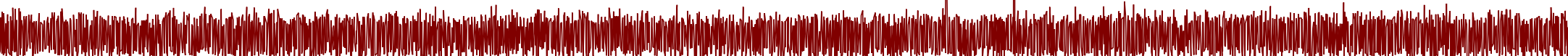

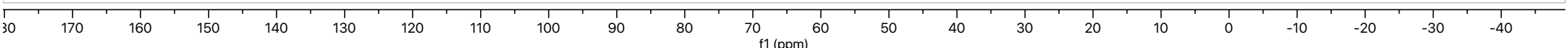




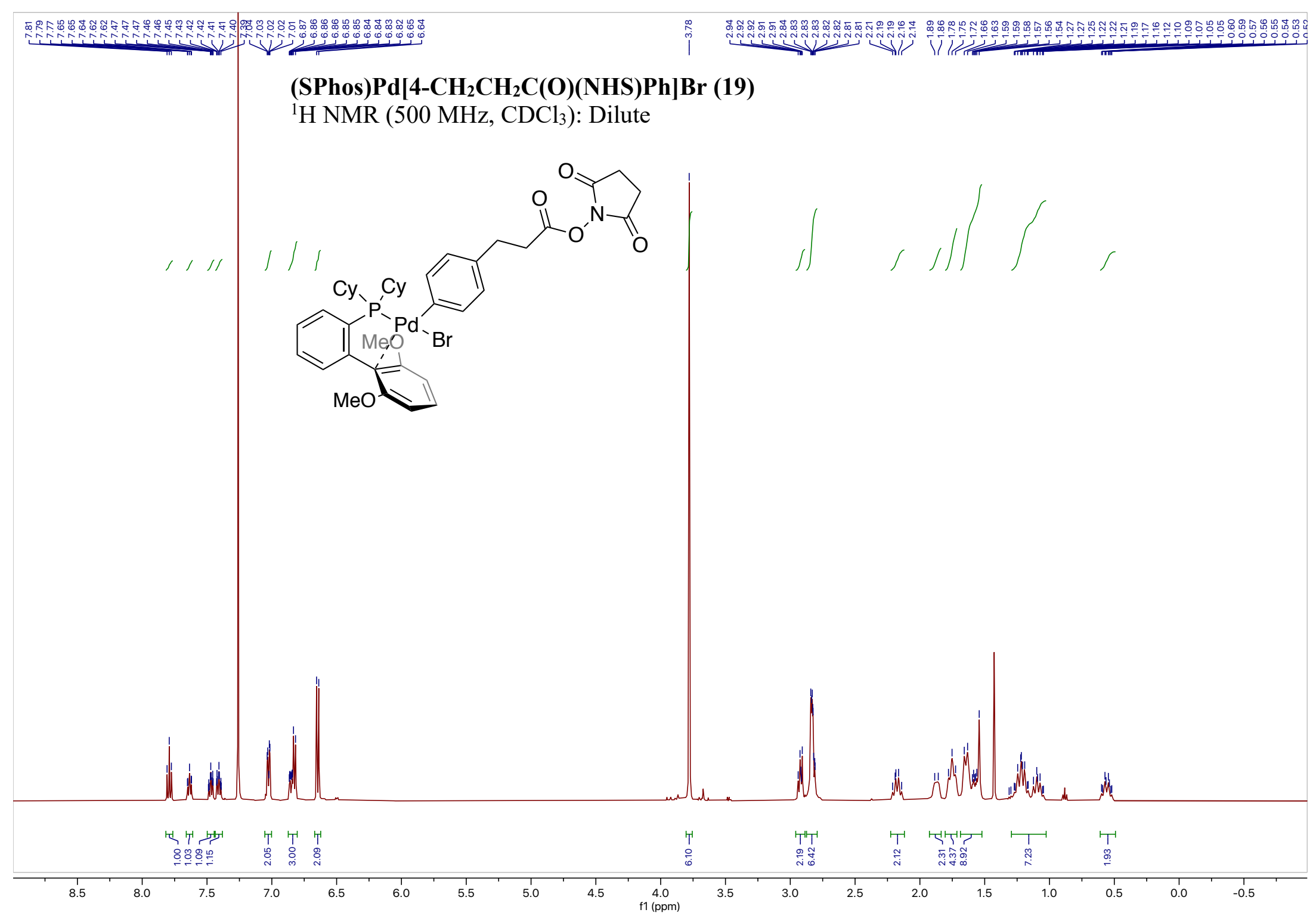




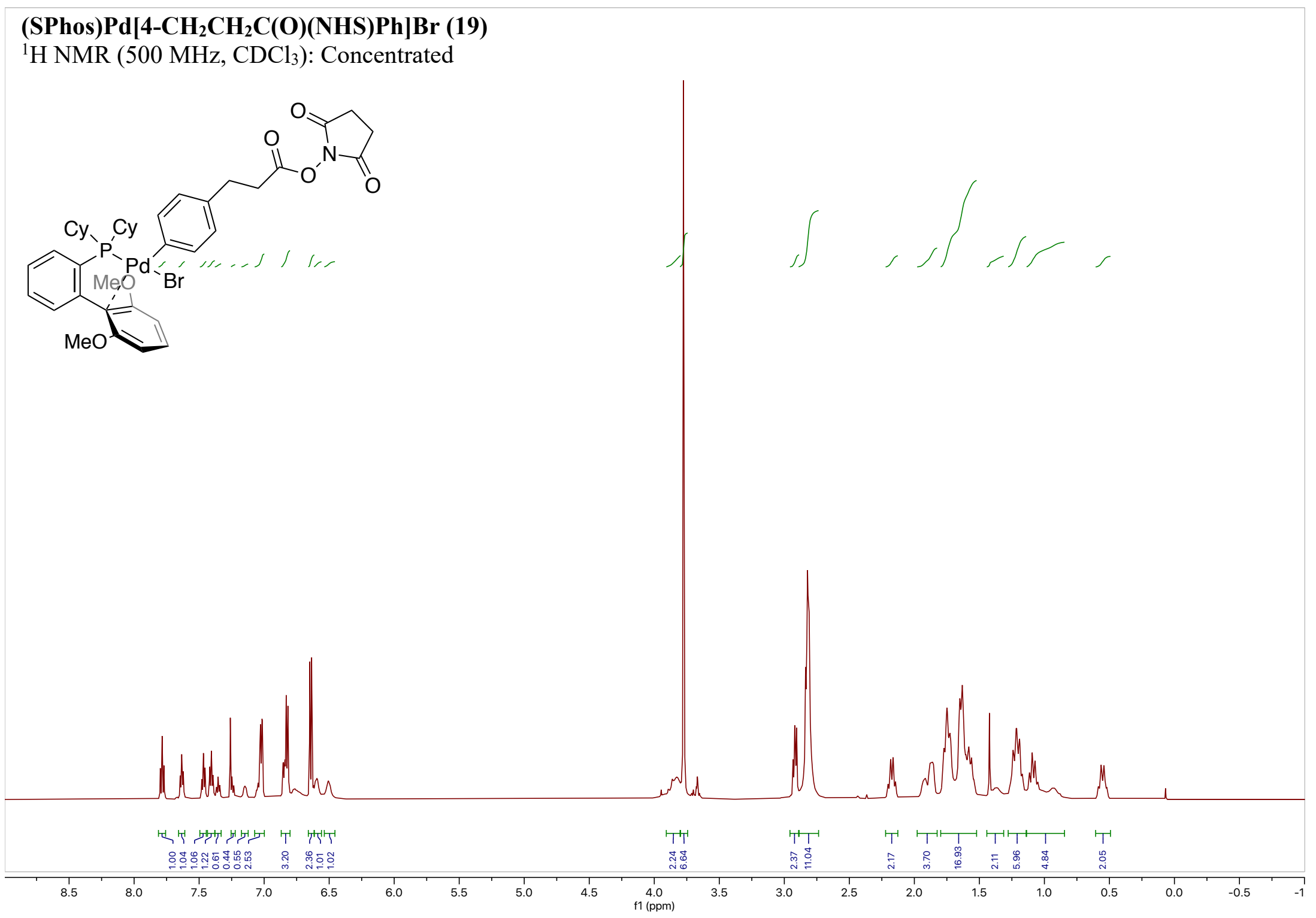

S122 


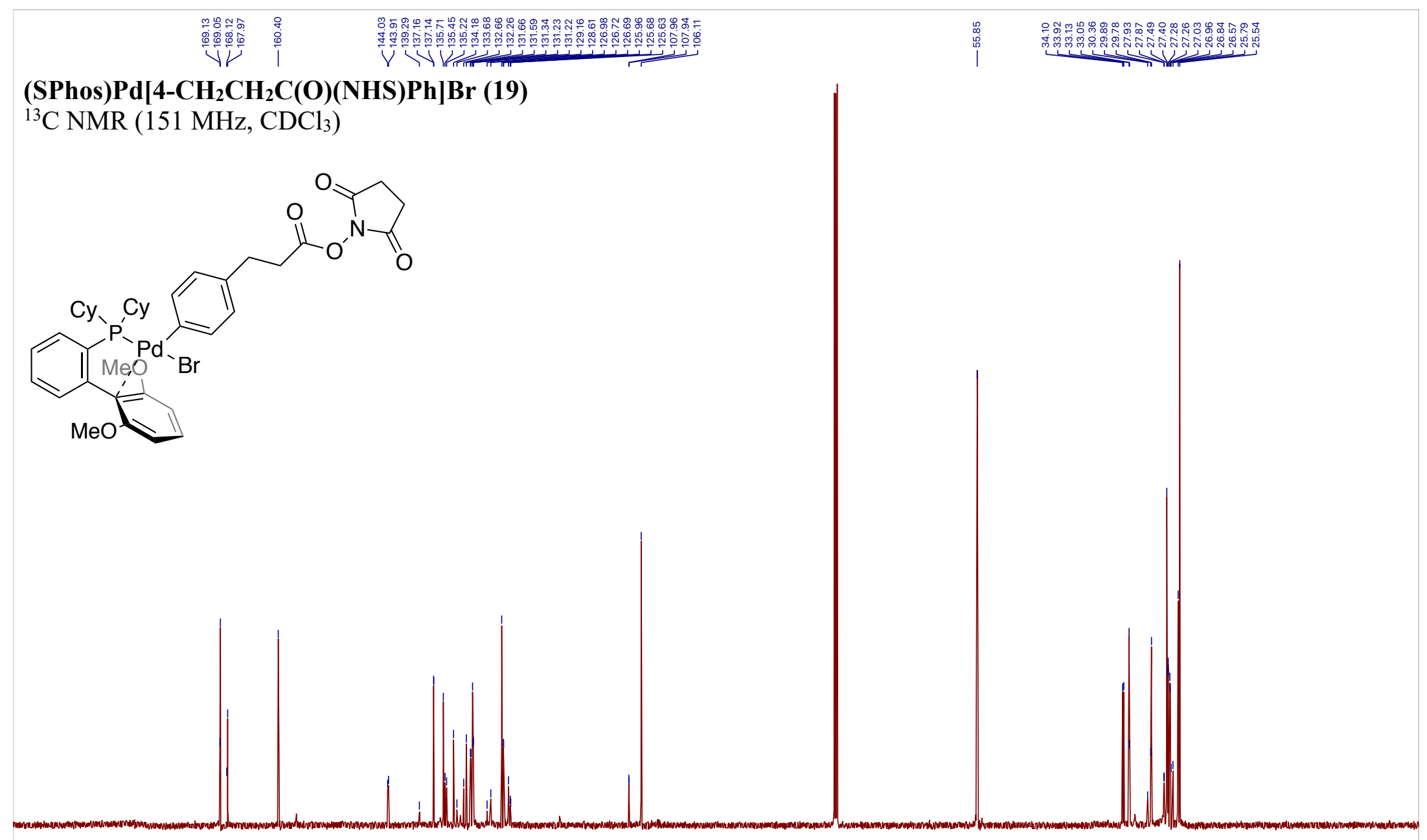


(SPhos)Pd[4-CH $\left.\mathrm{CH}_{2} \mathrm{C}(\mathrm{O})(\mathrm{NHS}) \mathrm{Ph}\right] \mathrm{Br}(19)$

${ }^{31} \mathrm{P}$ NMR $\left(162 \mathrm{MHz}, \mathrm{CDCl}_{3}\right)$

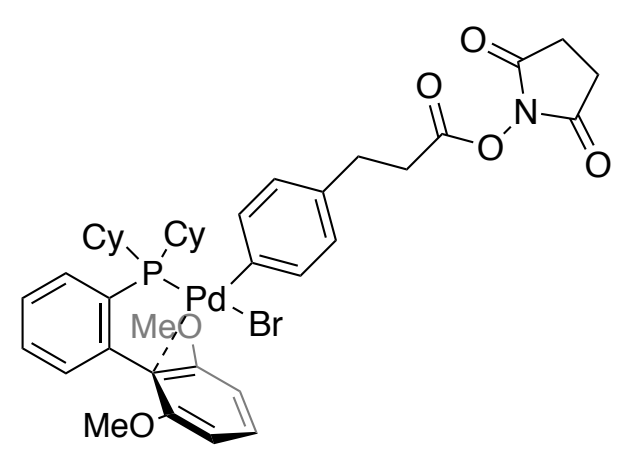

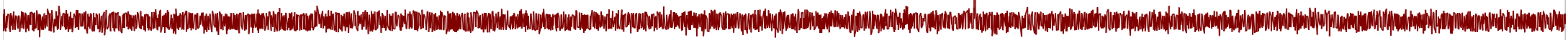

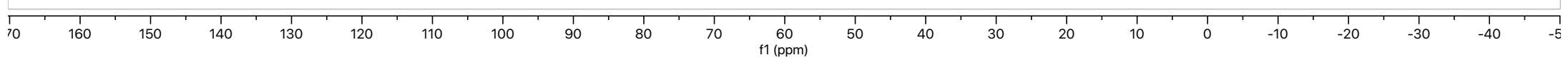


(sSPhos)Pd[(4-CO $\left.\left.\mathrm{C}_{2} \mathrm{C}_{6} \mathrm{~F}_{5}\right) \mathrm{Ph}\right] \mathrm{Br}$ (20)

${ }^{1} \mathrm{H} \mathrm{NMR}\left(600 \mathrm{MHz}, \mathrm{CD}_{2} \mathrm{Cl}_{2}\right.$ )
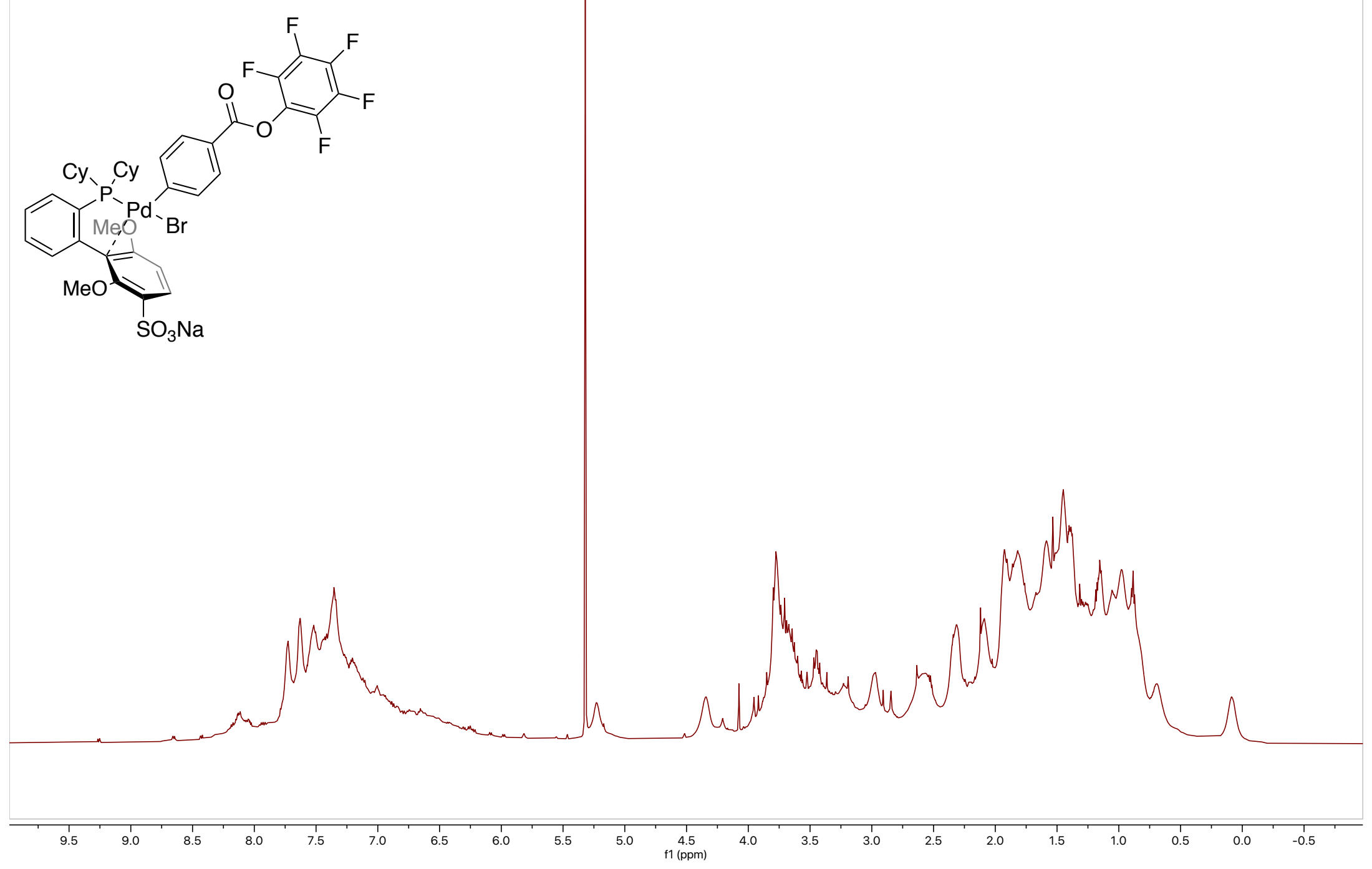


$$
\mathbb{Z}
$$


(sSPhos)Pd[(4-CO $\left.\left.{ }_{2} \mathrm{C}_{6} \mathrm{~F}_{5}\right) \mathrm{Ph}\right] \mathrm{Br}(20)$

${ }^{19} \mathrm{~F}$ NMR (565 MHz, $\mathrm{CD}_{2} \mathrm{Cl}_{2}$ )

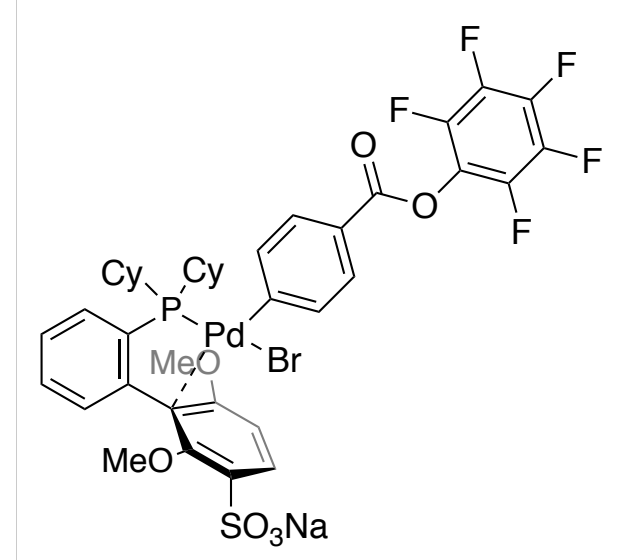

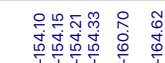

ن 
(sSPhos)Pd[(4-CO $\left.\left.\mathrm{C}_{2} \mathrm{C}_{6} \mathrm{~F}_{5}\right) \mathrm{Ph}\right] \mathrm{Br}(20)$

${ }^{31} \mathrm{P}$ NMR (202 MHz, $\mathrm{CD}_{2} \mathrm{Cl}_{2}$ )
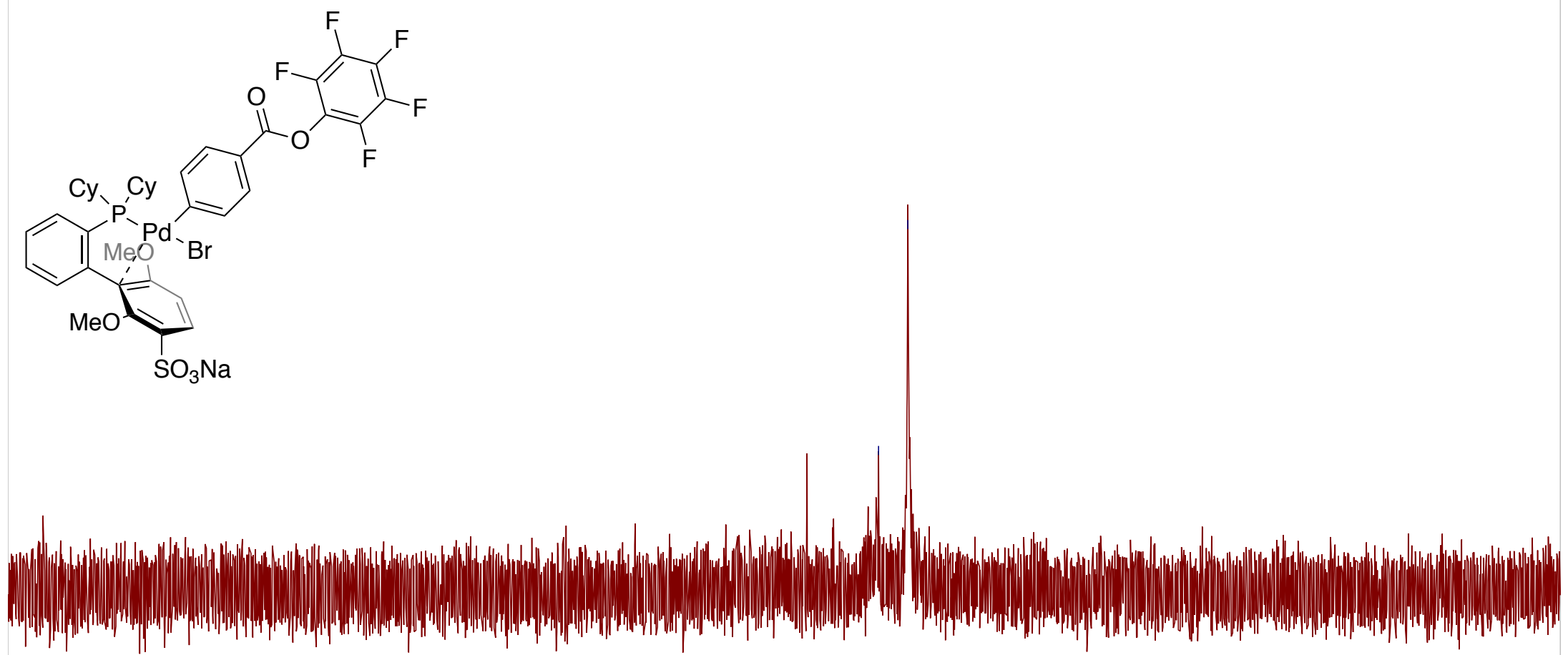

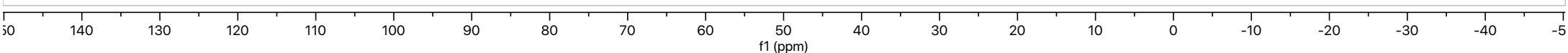




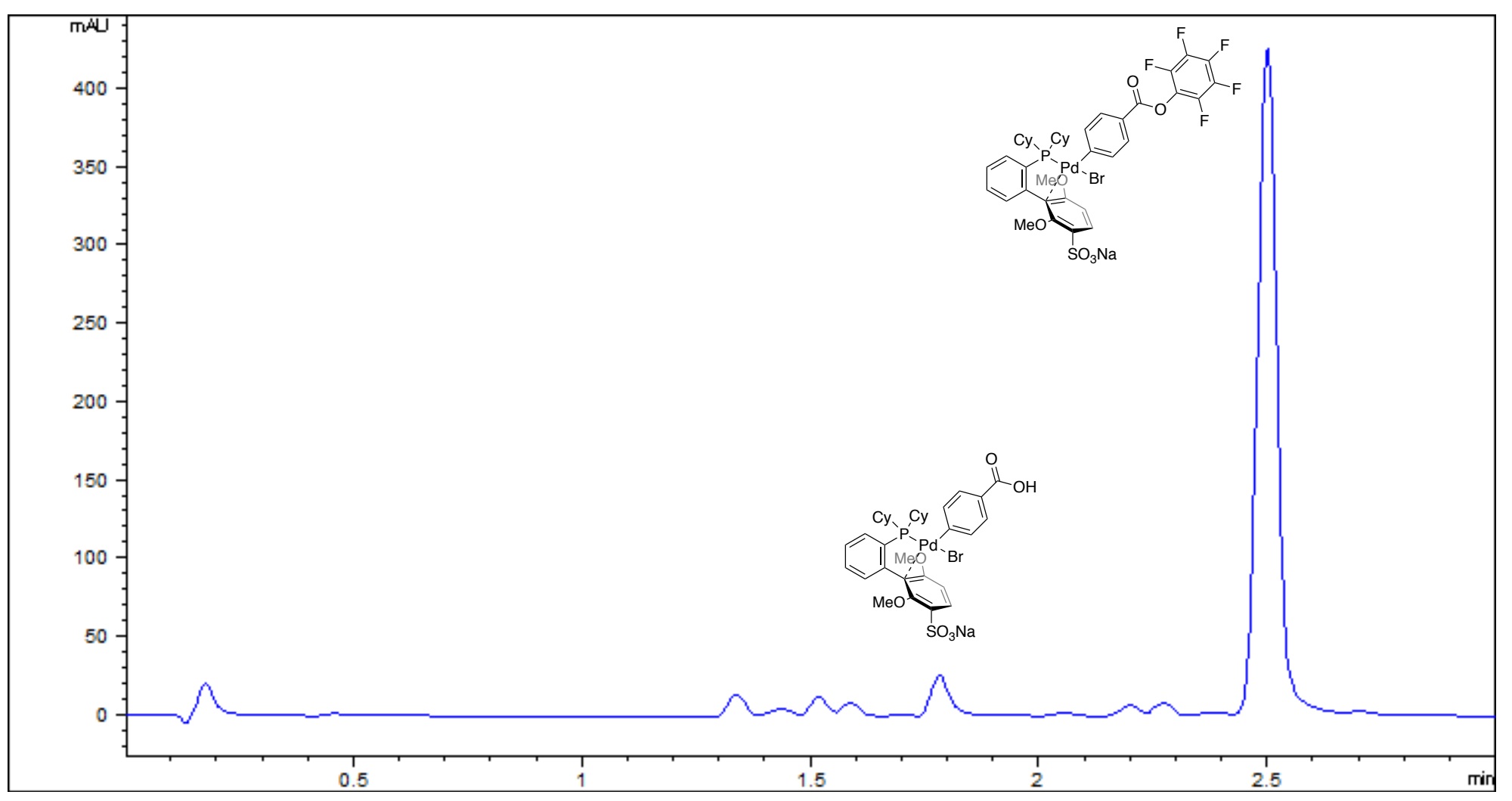




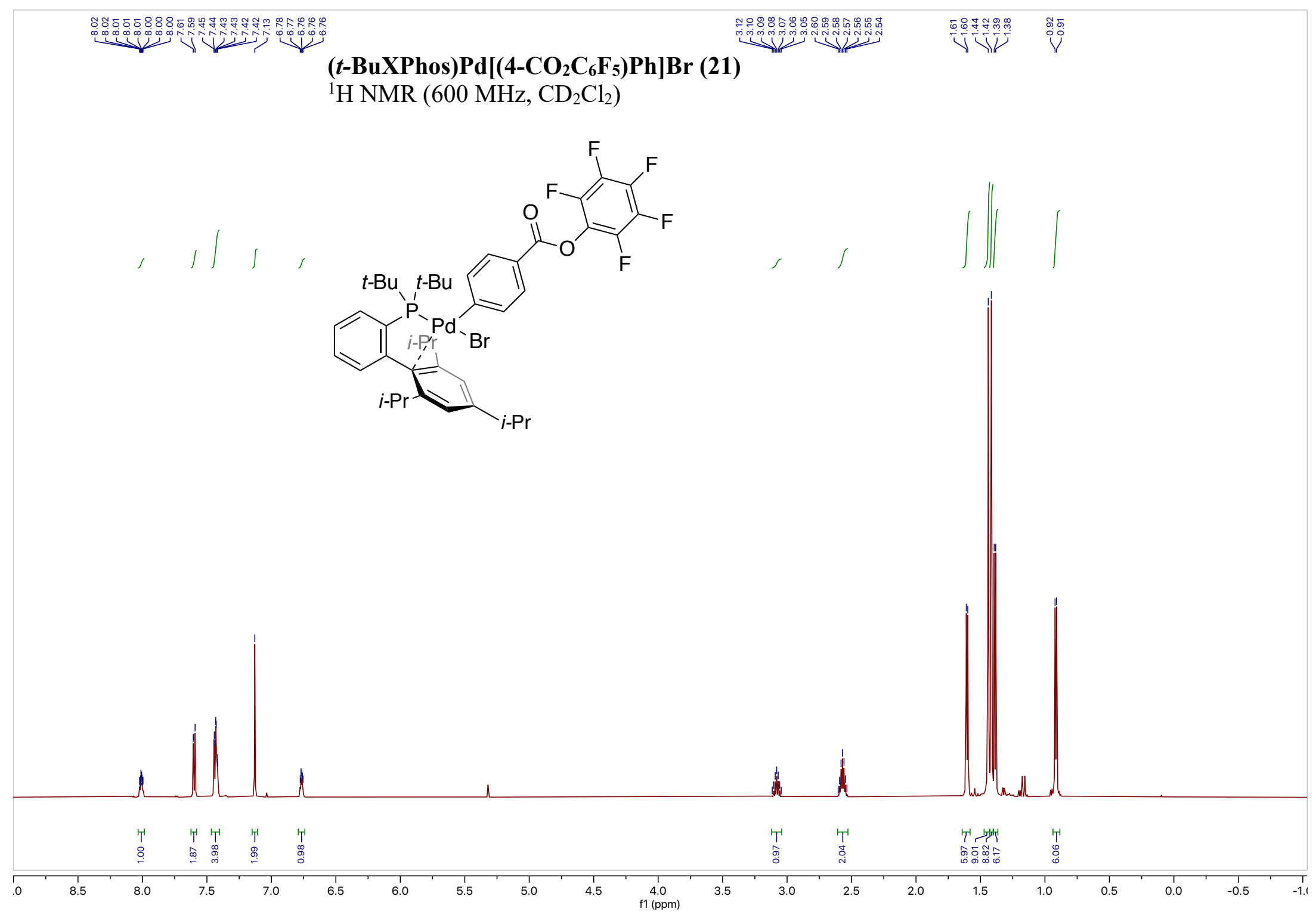







(t-BuXPhos)Pd[(4-CO $\left.\left.\mathrm{C}_{2} \mathrm{C}_{5}\right) \mathrm{Ph}\right] \mathrm{Br}$ (21)

${ }^{19} \mathrm{~F}$ NMR (565 MHz, $\mathrm{CD}_{2} \mathrm{Cl}_{2}$ )

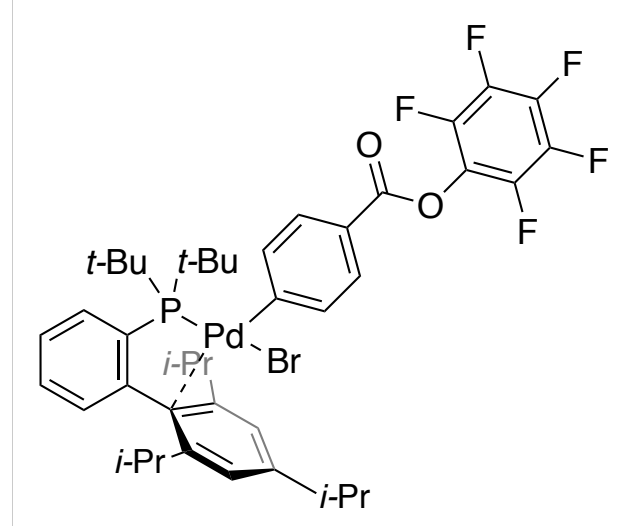




\section{(t-BuXPhos)Pd[(4-C $\left.\left.\mathrm{CO}_{2} \mathrm{C}_{6} \mathrm{~F}_{5}\right) \mathrm{Ph}\right] \mathrm{Br}$ (21)}

${ }^{31} \mathrm{P}$ NMR (203 MHz, $\mathrm{CD}_{2} \mathrm{Cl}_{2}$ )
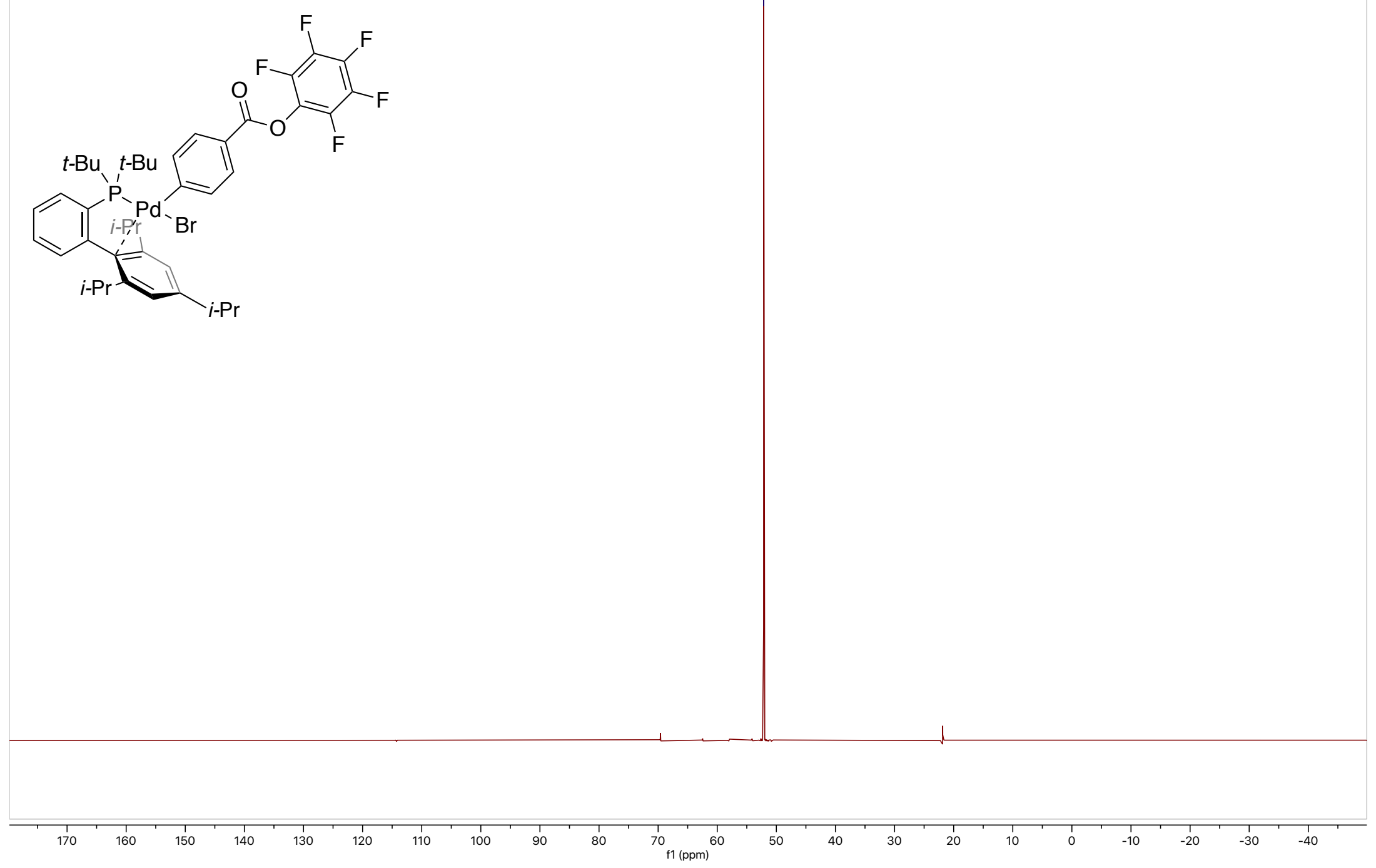


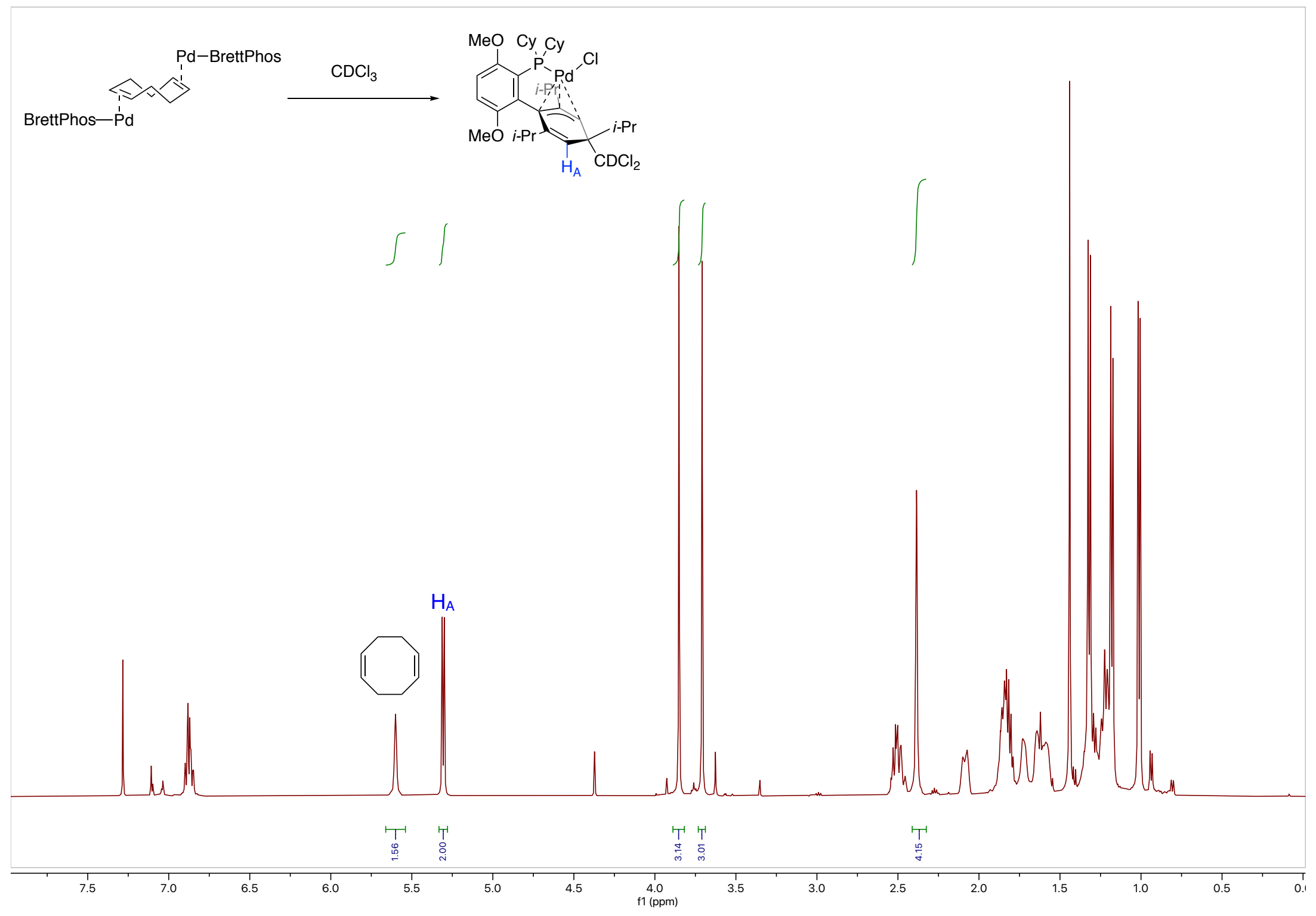




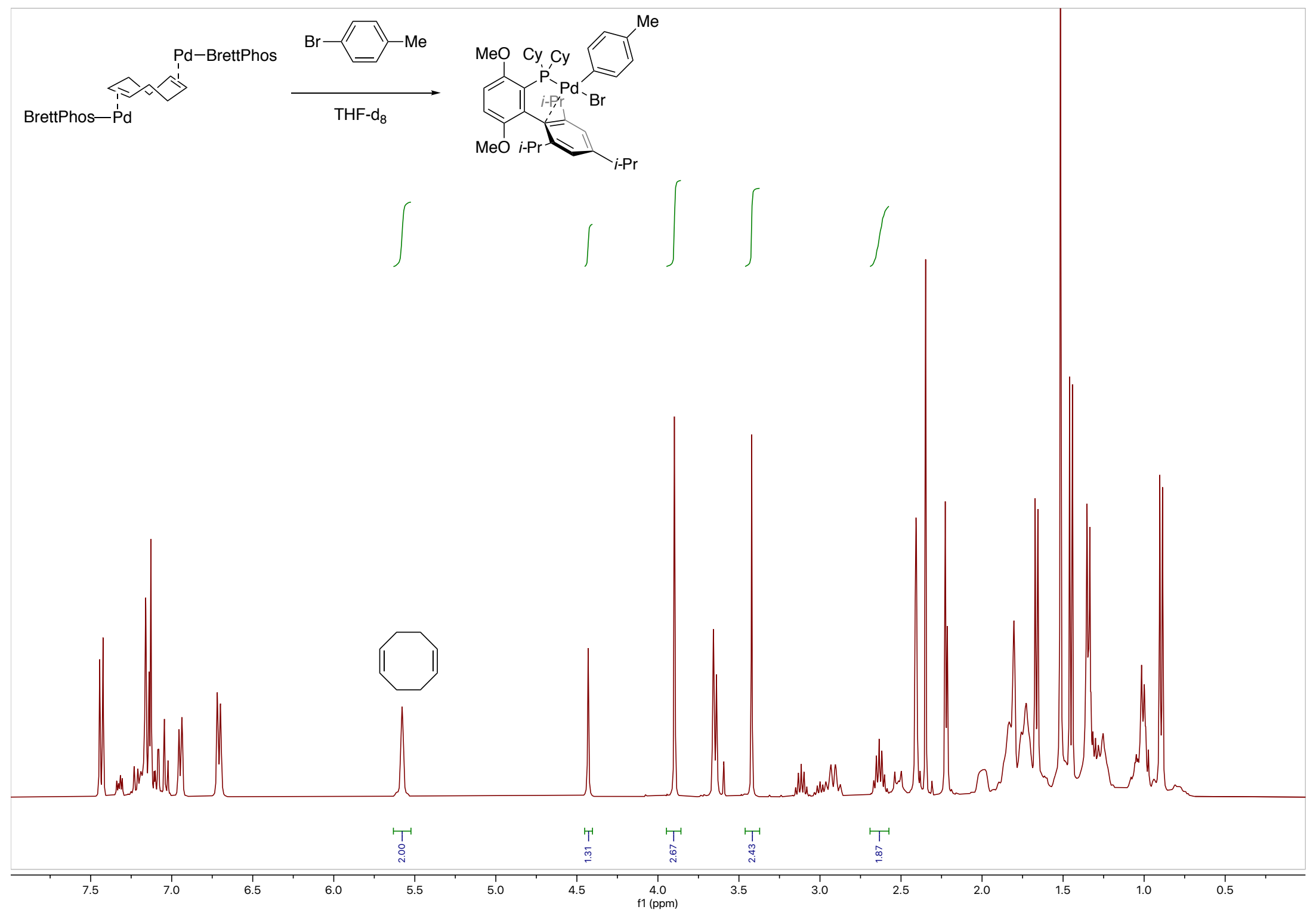




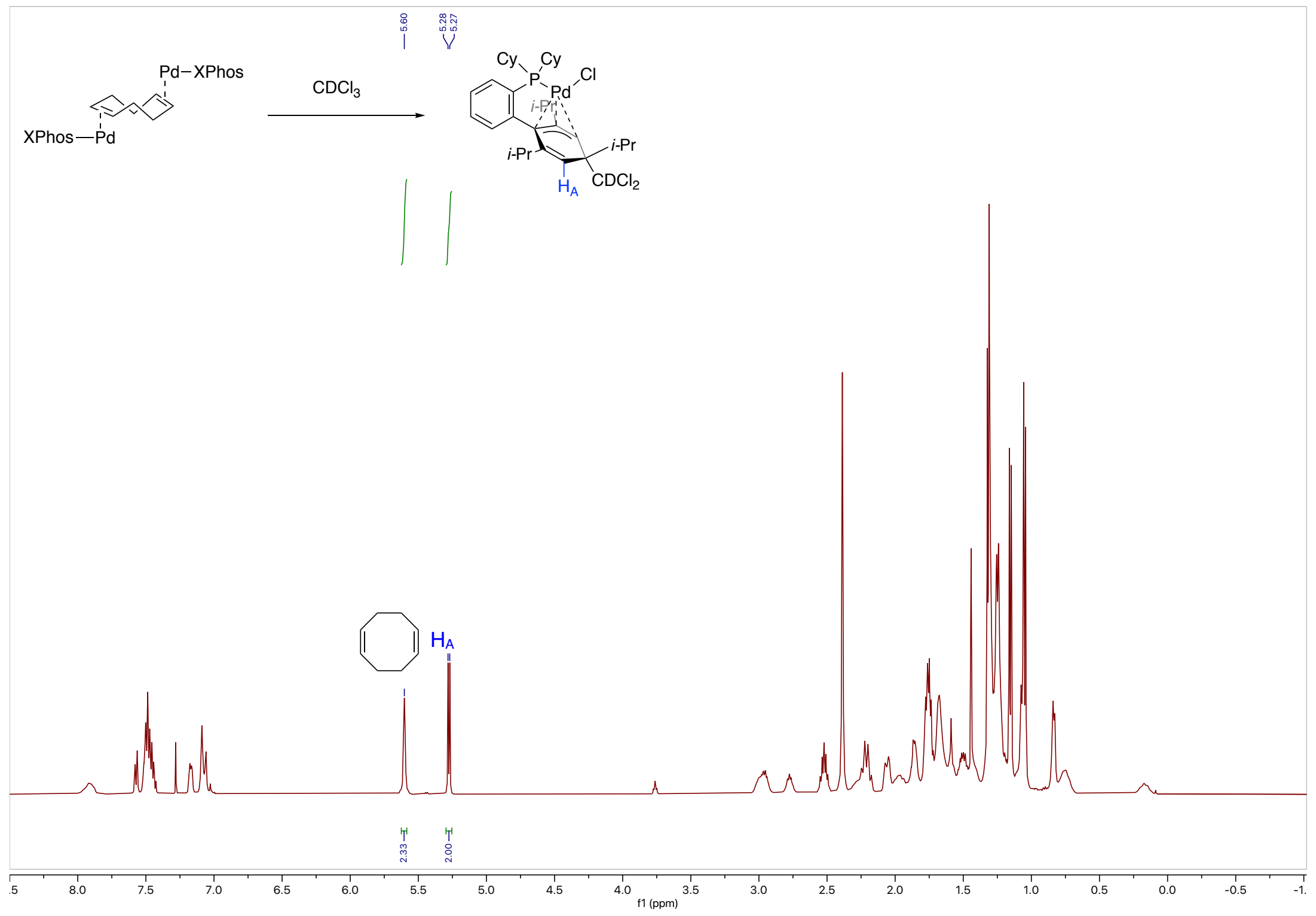

S136 


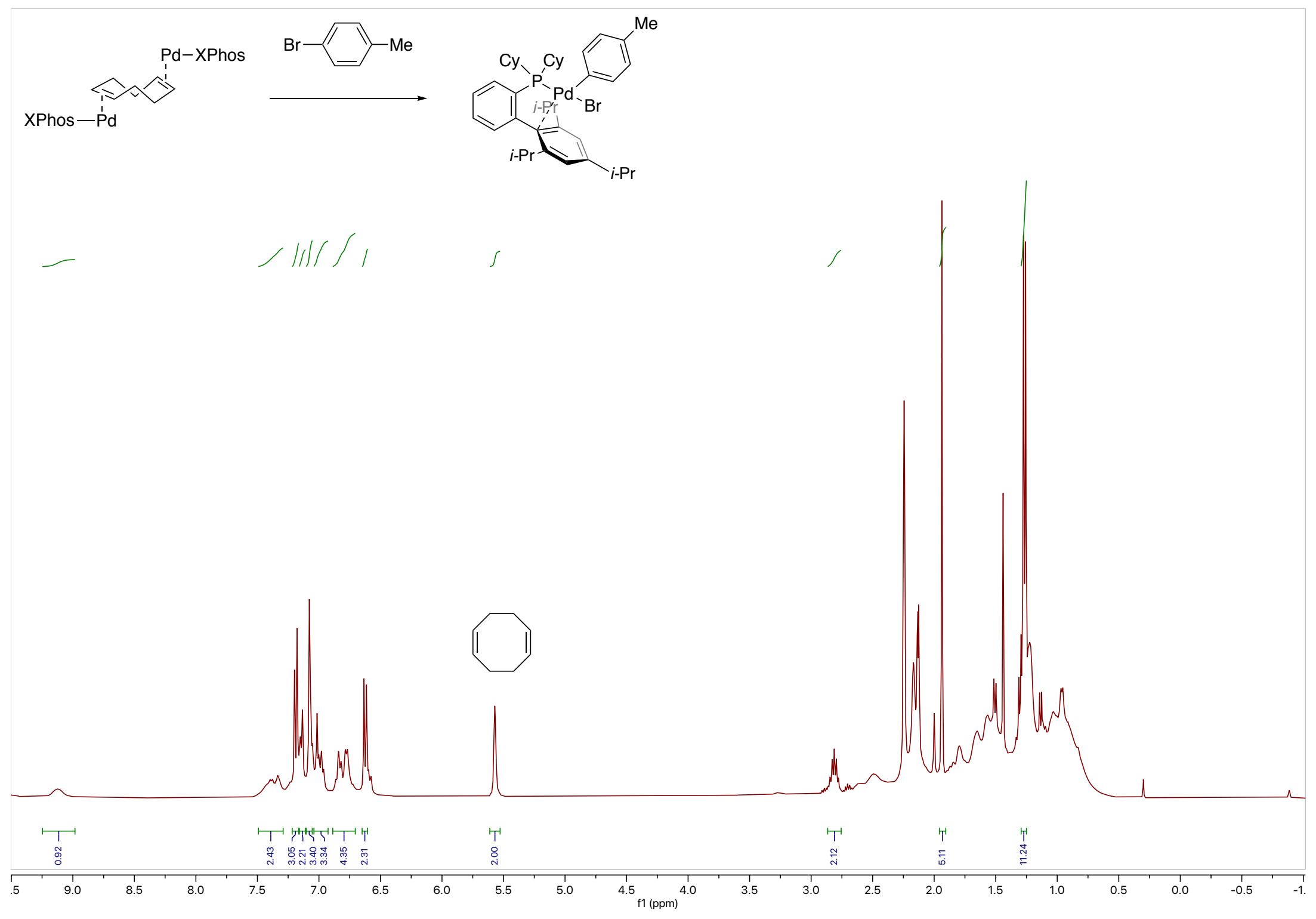




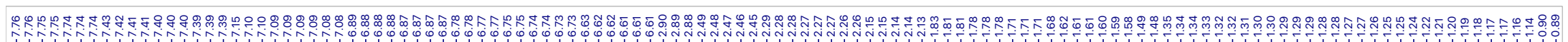

(XPhos)Pd( $\left.\mathrm{CH}_{2} \mathrm{CMe}_{2} \mathrm{Ph}\right)(25)$

${ }^{1} \mathrm{H}$ NMR $\left(600 \mathrm{MHz}, \mathrm{CD}_{2} \mathrm{Cl}_{2}\right)$

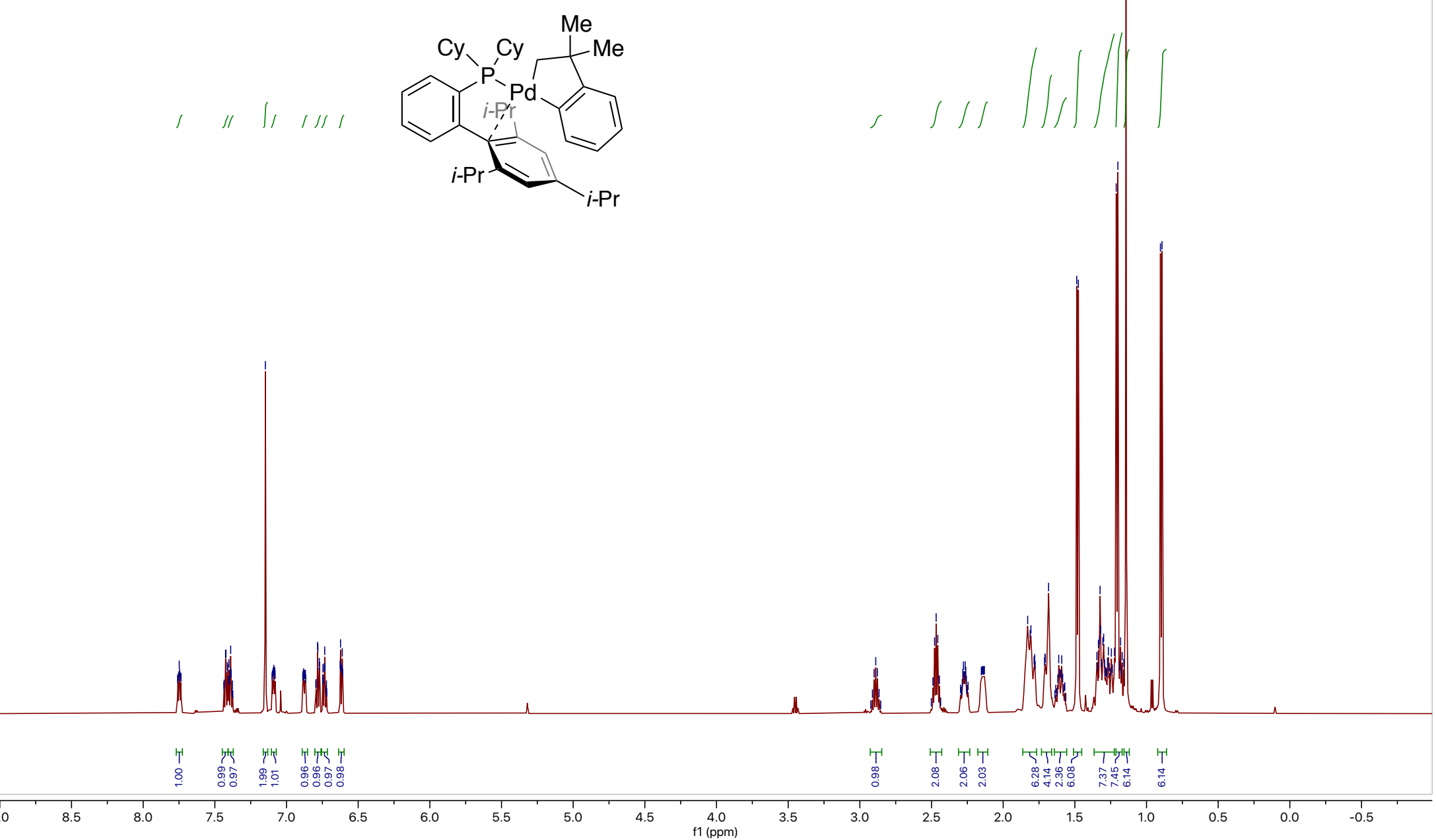




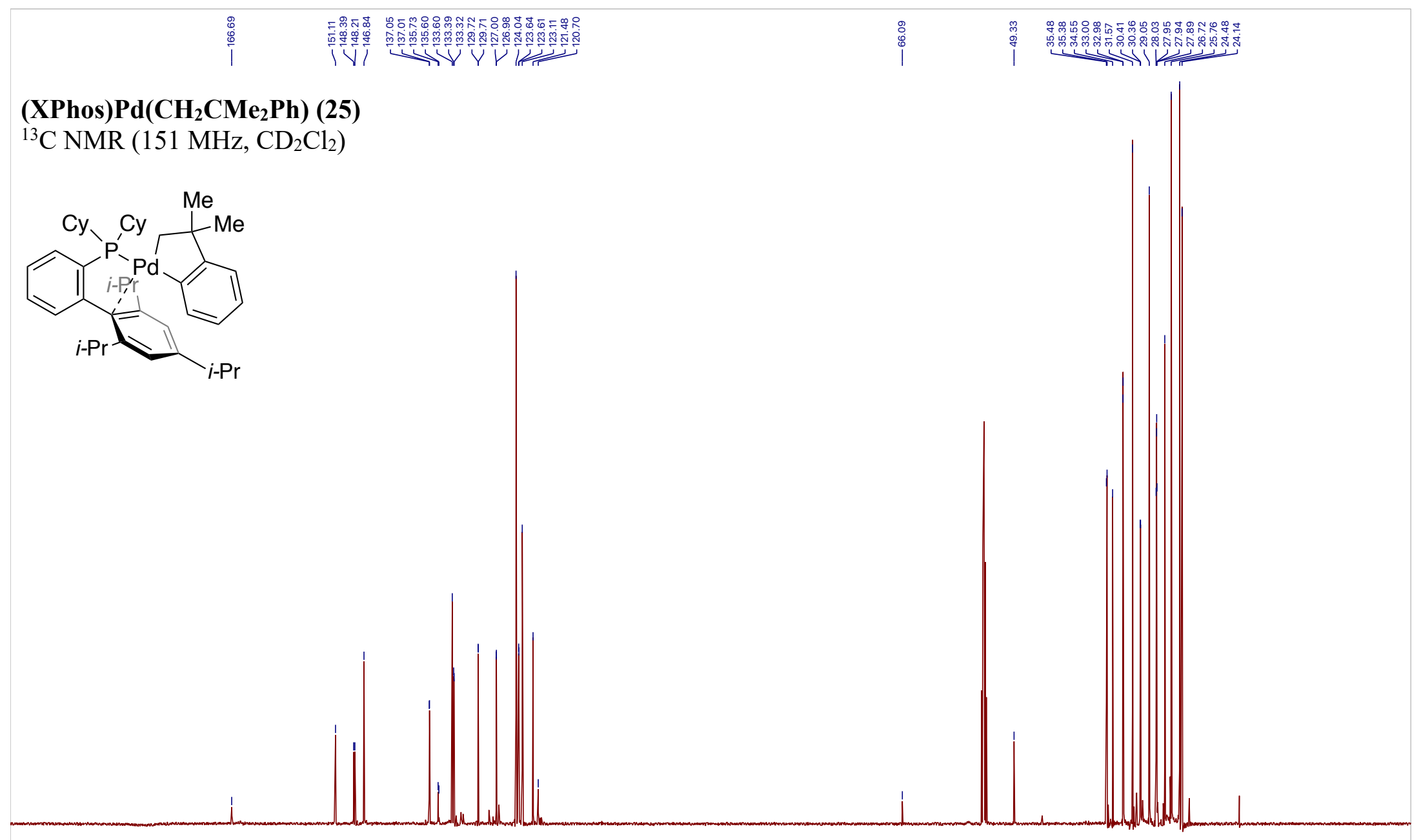




\section{(XPhos)Pd( $\left.\mathrm{CH}_{2} \mathrm{CMe}_{2} \mathrm{Ph}\right)$ (25)}

${ }^{31} \mathrm{P}$ NMR $\left(162 \mathrm{MHz}, \mathrm{CD}_{2} \mathrm{Cl}_{2}\right)$

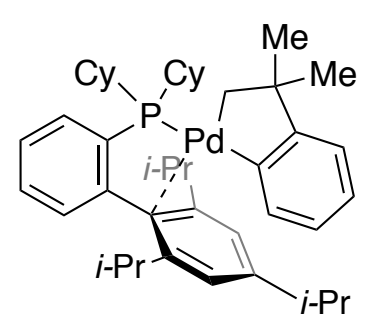






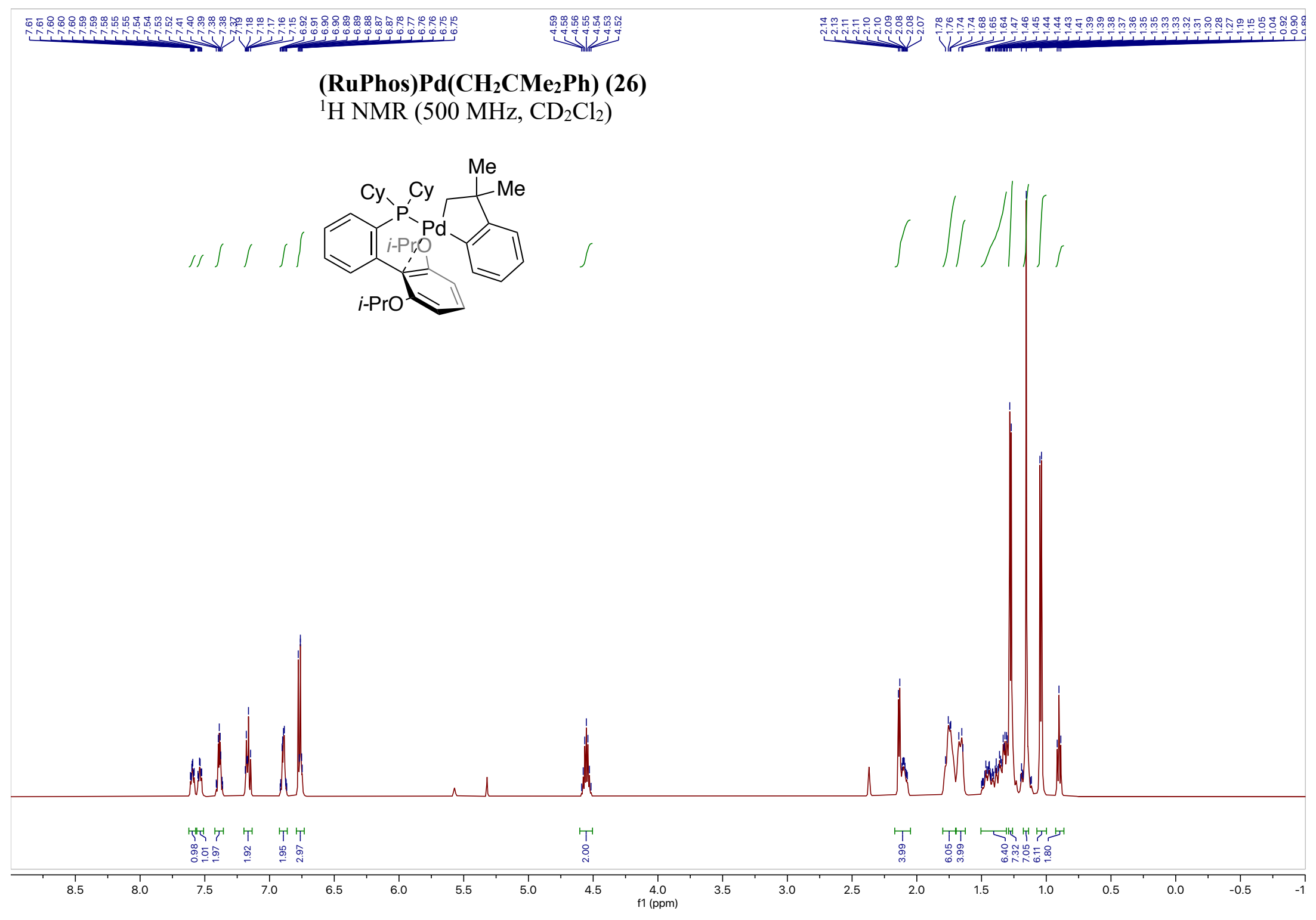




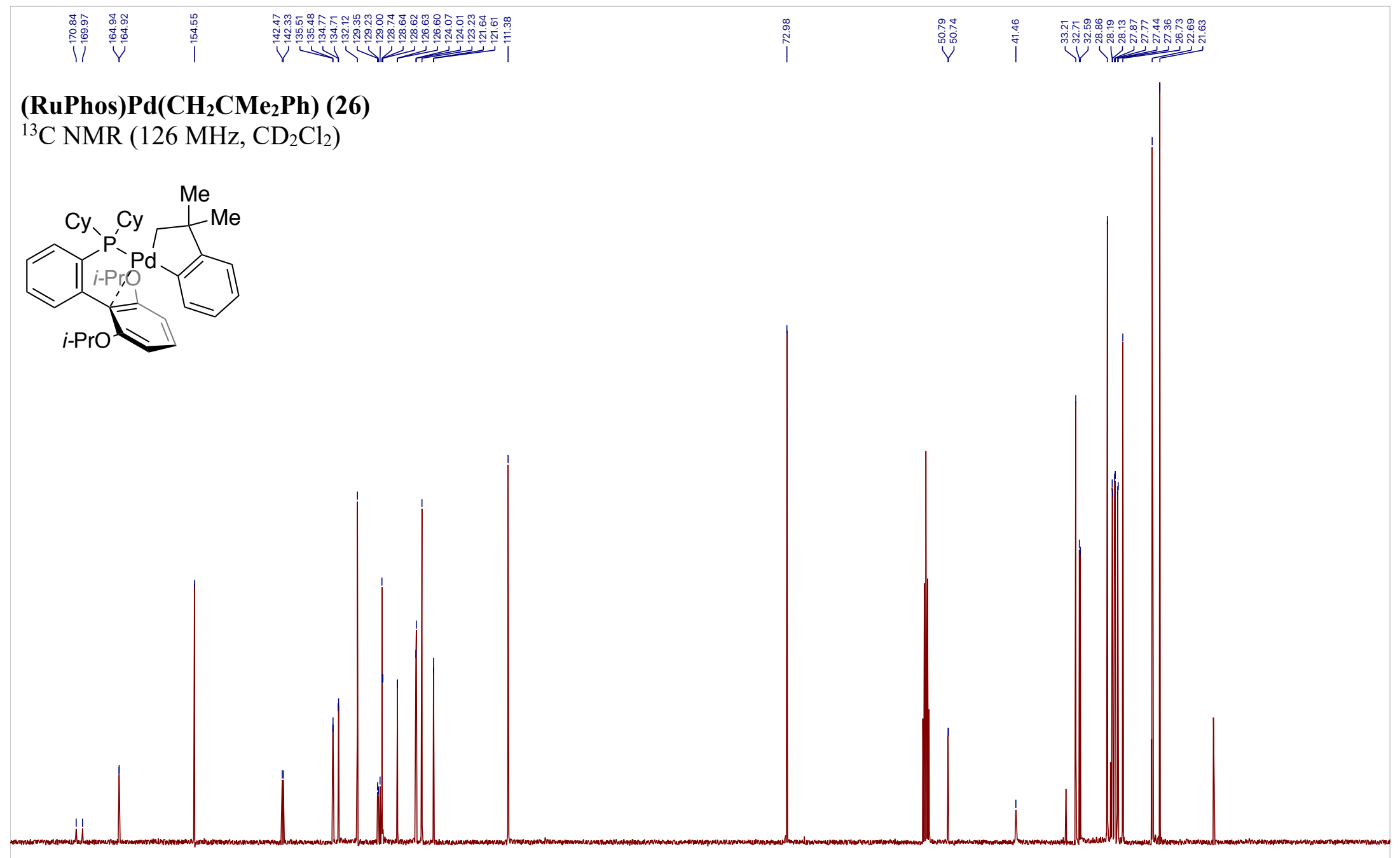


(RuPhos)Pd( $\left.\mathrm{CH}_{2} \mathrm{CMe}_{2} \mathrm{Ph}\right)$ (26)

${ }^{31} \mathrm{P}$ NMR (203 MHz, $\mathrm{CD}_{2} \mathrm{Cl}_{2}$ )
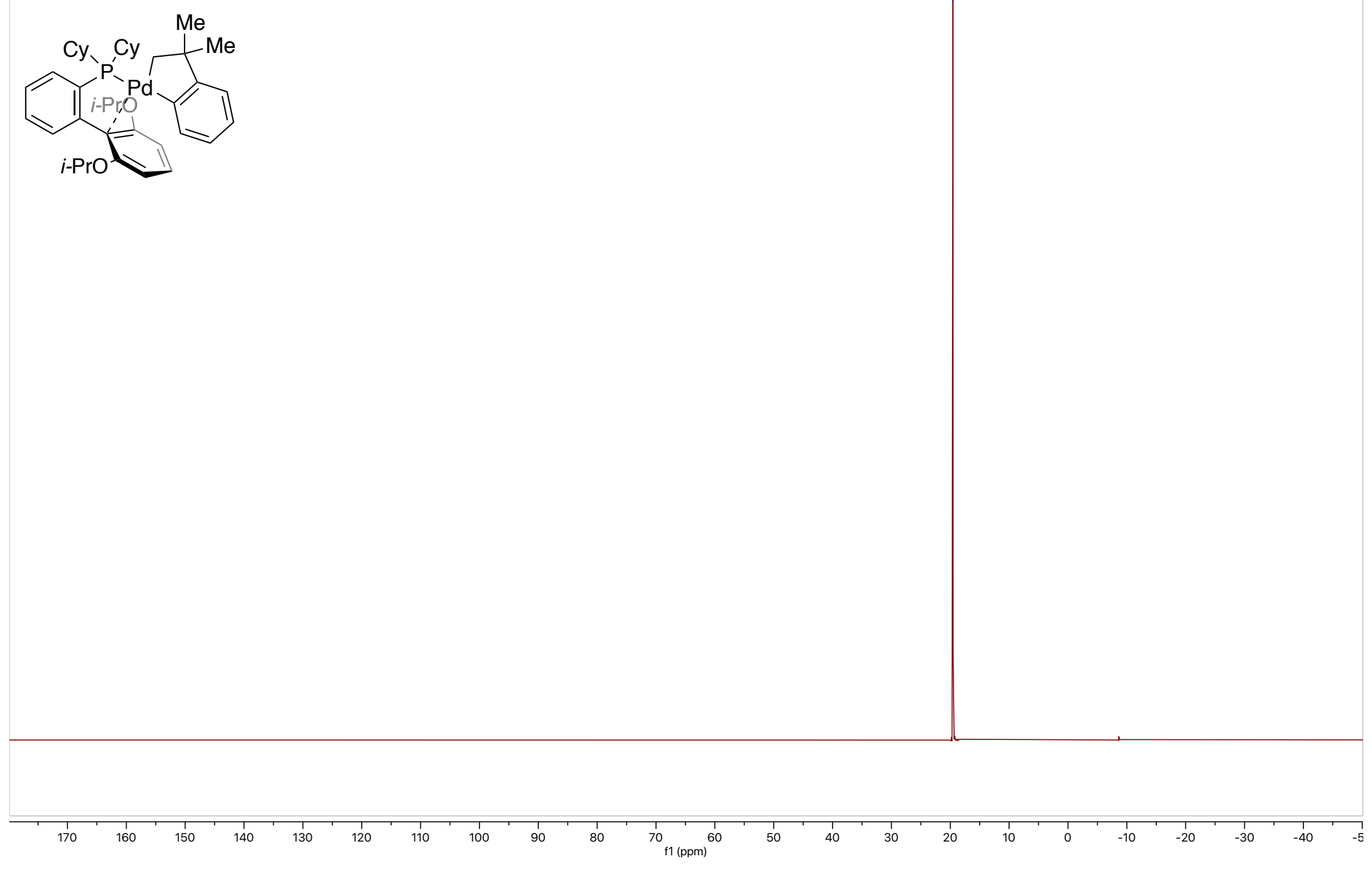


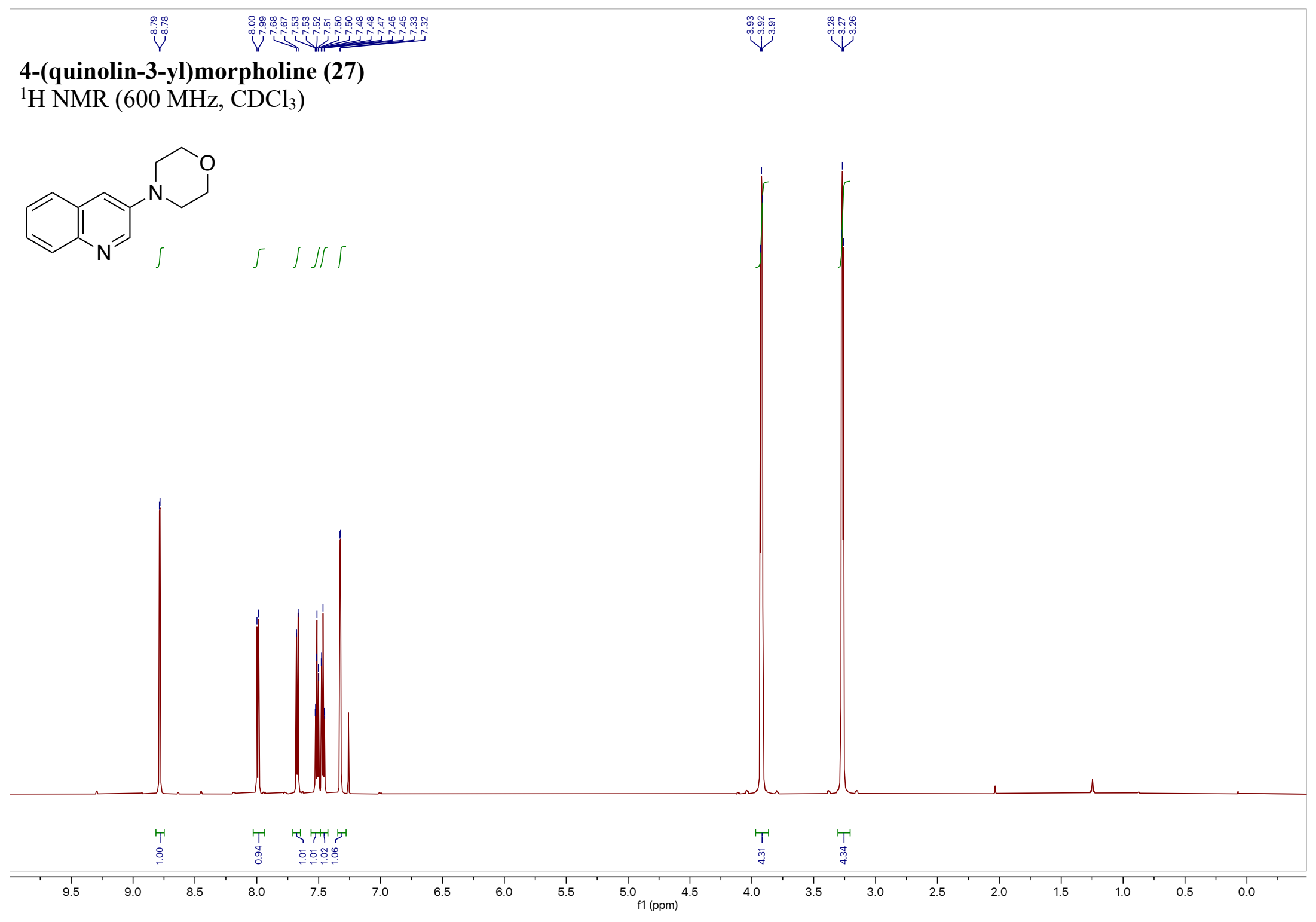




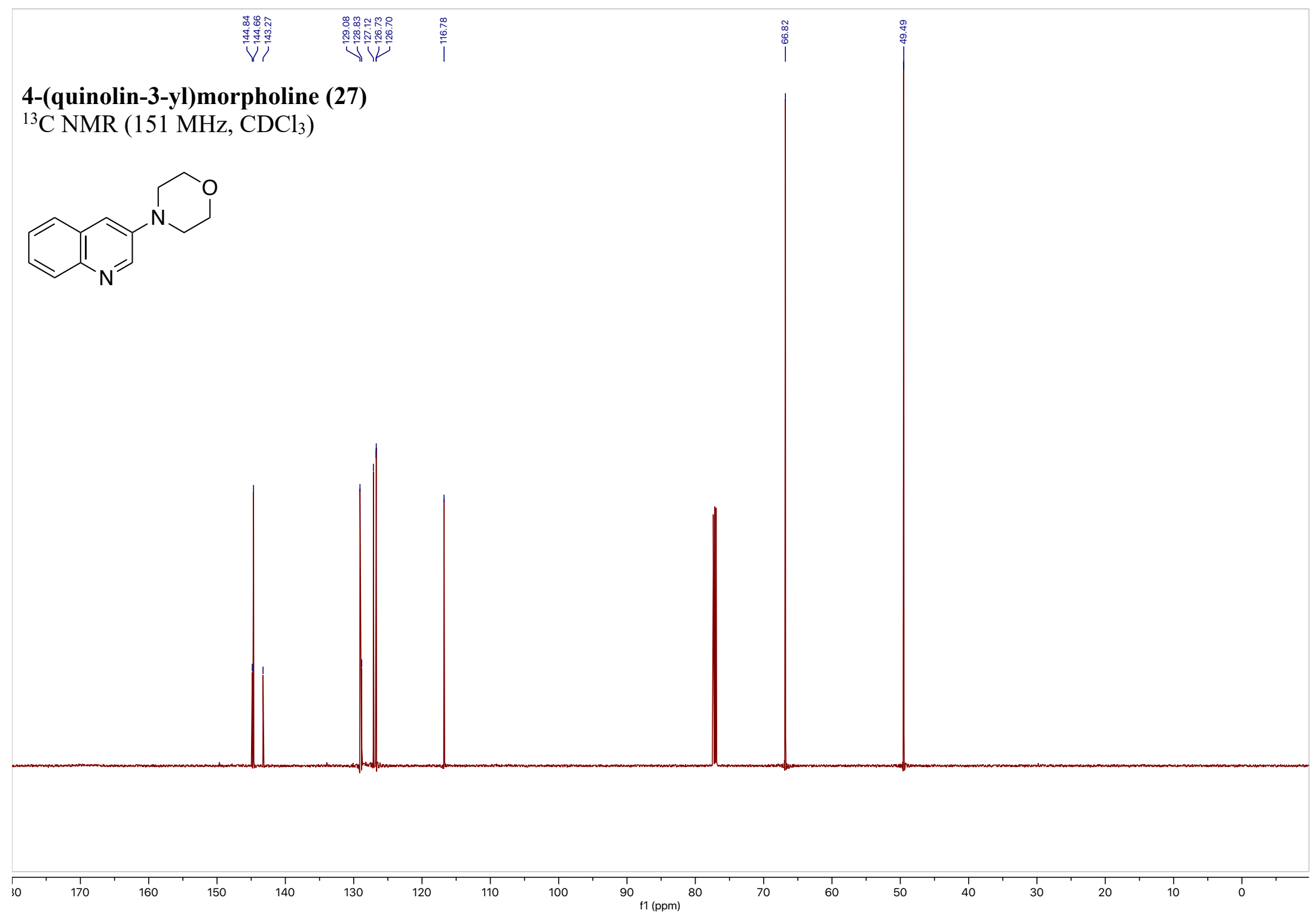

S145 